\title{
VIII. «An Unconditional Ally»: Die Etablierung Irans als Stellvertreter westlicher Interessen in der Nahostregion, 1967-1975
}

VIII. 1. Aufrüstung ohne Grenzen? Der Weg zum «Blankoscheck» von 1972

Im August 1968 nahm Botschafter Armin Meyer an einer Gedenkfeier zum 15. Jahrestag des 28. Mordād, des Sturzes des ehemaligen Ministerpräsidenten Mosaddeg und der Rückkehr Mohammad Rezās auf den Thron, ausgerichtet von der Tāğ-o-1-Moluk, der Mutter des Schahs, teil. Meyer registrierte die Abwesenheit der wichtigsten Akteure der Begebenheiten von 1953 trotz einer Gästezahl von einigen Hundert. Statt der damaligen Protagonisten dominierten jetzt servile Staatsbeamte und die unausweichliche Jeunesse dorée Teherans die Veranstaltung. Es sei eine heitere Feier mit spektakulärem Feuerwerk gewesen, berichtete der Botschafter: „But few had a clue as to what was being celebrated."1

Die Schilderung Meyers ist bezeichnend für den Wandel des Zeitgeists im Iran der späteren 1960er Jahre, geprägt durch die vom Wirtschaftsboom ausgelöste Aufbruchsstimmung, die Konzentration auf persönliches Weiterkommen und die eigene Karriere und die Fokussierung auf die Zukunft anstelle der Vergangenheit. Mosaddeg selbst war am 5. März 1967 im Alter von 87 Jahren im Ahmadābāder Hausarrest verstorben. Das Regime erlaubte nur eine kurze Mitteilung in der nationalen Presse und verweigerte eine Beisetzung über den familiären Rahmen hinaus. Die Botschaft registrierte erstaunt, Mosaddegs Tod rufe kaum eine Reaktion seitens der Bevölkerung hervor: ,We are struck by the general unconcern over $\{$ the; R.P. $\}$ death of one of $\{$ the $\}$ great personalities of Persian history."2 Das Desinteresse am früher landesweit verehrten Politiker war für die Botschaft Ausdruck des dominierenden Interesses am Hier und Jetzt bei den meisten Iranern. Zudem war Mosaddeg immer die Symbolfigur des „klassischen“ nationalistischen Widerstands gegen die Schah-Diktatur gewesen, der nunmehr nur noch rudimentär anzutreffen sei. Im April 1969 versicherte die Botschaft, dass das progressive

\footnotetext{
1 Vgl. Tehran \#A-782, 21.8.68, POL 15-1 IRAN, CF 67-69; Meyers ironisch gefärbte Schilderung der Feierlichkeiten an diesem Tag enthält zudem eine Reihe von Referenzen zu den Protagonisten des Coups von 1953. „Schwartzy“ (Norman Schwarzkopf Sr.) sei bereits verstorben, Loy Henderson schreibe seine Memoiren und Kermit Roosevelt habe sich pekuniär einträglicheren Aktivitäten zugewandt. Von den Anwesenden wisse nur der gegenwärtige Außenminister Ardešir Zāhedi mehr über die damaligen Ereignisse (,[...] he had in those days been a frequenter, as much as any employee, of the basement of the Chancery of the American Embassy on Takhte Jamshid Street."; gemeint ist die CIA-Station in der Botschaft). In den internen CIA-Geschichten wird Ardešir Zāhedi allerdings nur als Kontaktmann zu seinem Vater, nicht als aktiver Agent erwähnt. Vgl. CIA [Donald N. Wilber], Clandestine Service Service History, S. 4; CIA History Staff, The Battle for Iran, S. B-5f.

${ }^{2}$ Tehran \#3529, 8.3.67; vgl. Tehran \#A-493 to State, 9.3.67, POL 6 IRAN, CF 67-69, RG 59, NACP; offenbar hatte Howejdā den Schah um eine angemessene Beisetzungsfeier für den ehemaligen Premier gebeten. Mohammad Rezā habe dies strikt abgelehnt (,,[...] he wished to erase every trace of Mosadeq in the land."); vgl. DE BELLAIGUE, Patriot of Persia, S. 270; die Verachtung gegenüber Mosaddeg zeigte sich in seiner Antwort auf die Frage eines französischen Journalisten nach dessen Befinden. Der Ex-Premier sei guter Gesundheit und gehe seinem Lieblingssport nach: ,riding donkeys“; ebd., S. 266; die bestehende Verunsicherung Mohammad Rezās aufgrund der Anerkennung Mosaddeg als iranischer Patriot äußerte sich auch in einer diffamierenden Darstellung in den „offiziellen“ Memoiren des Monarchen. Vgl. PAHLAVI, Mission for My Country, S. 82-110.
} 
Reformprogramm Mohammad Rezās im Zusammenspiel mit den effizienten Sicherheitsorganen, ,[...] destroyed all organized political opposition in Iran.“3 Angesichts der Auflösung der oppositionellen Strukturen und der augenfälligen ökonomischen und außenpolitischen Erfolge des Schahs gab es aus Sicht der Teheraner Botschaft offenbar keinerlei Grund mehr für Besorgnis.

Unter der Oberfläche aber war ein Rumoren zu vernehmen. Die ersten Ausläufer der sich nun neu formierenden nationalistisch-religiösen Revolutionsbewegung waren bereits Ende der 1960er Jahre sichtbar. Zwar gab es in der Tat kaum existierende oppositionelle Strukturen, abgesehen von den bald darauf aktiv werdenden Terrorgruppen, und, mit Ausnahme Khomeinis im irakischen Exil, keine glaubhaften Führungsfiguren. Die ideellen Grundlagen aber waren durchaus bereits vorhanden. Die Gegnerschaft gegen die außenpolitische Strategie des Schahs brach sich im Mai 1968 unerwartet Bahn im Kontext der Asienmeisterschaft im Fußball, die in diesem Jahr in Iran ausgetragen wurde. Im entscheidenden Spiel entthronte der Gastgeber Iran den amtierenden Asienmeister Israel mit einem umkämpften 2:1-Sieg. Die Botschaft berichtete über die noch nie dagewesene Fußballbegeisterung in Iran und den allgemeinen Enthusiasmus über die errungene Meisterschaft, Mohammad Rezā eingeschlossen. Am Rande aber registrierte man, offenkundig irritiert, die Begleiterscheinungen während des Spiels gegen die Israelis. Bereits im Vorfeld und auch während des Spiels waren antiisraelische bzw. auch antisemitische Sprechchöre zu vernehmen und nach dem Sieg randalierte eine radikalisierte Fangruppe in einem jüdischen Viertel im Süden Teherans. Die Botschaft meldete: „The match seemed to assume, for the masses at least, some aspects of a power struggle between Iran as a Moslem country and Israel as a country which had defeated Moslem armies. “4 Mit dem Spiel, von Millionen im Fernsehen verfolgt, avancierte Fußball zum Massenphänomen in Iran. Gleichzeitig blieb die Legende des Siegs über Israel als früher Ausdruck kollektiver Überzeugungen, die zehn Jahre später einen wichtigen Bestandteil des revolutionären Selbstverständnisses von Millionen Iranern repräsentieren sollten. ${ }^{5}$

Am Ende der Dekade aber war Iran aus amerikanischer Sicht der Vorbildstaat schlechthin für das eigene Engagement an der Peripherie. Die CIA betitelte eine Lageeinschätzung zu Iran im Oktober 1971 - mit gebremster Süffisanz - „Nothing Succeeds Like a Successful Shah““ 6 Der Schah - noch viel stärker als bereits zuvor spiegelt sich hier die Gleichsetzung von Monarchen und Staat - verfüge in der Tat über vieles, worauf er mit Recht stolz sein könne. Wenig erinnere

\footnotetext{
${ }^{3}$ Tehran \#A-184, 21.4.69, POL 15-4 IRAN, ebd., auch in Asnād 20, 1, S. 27-39.

${ }^{4}$ Tehran \#A-625, 28.5.68, CUL 15 IRAN, CF 67-69; eine Satirezeitschrift druckte eine Karikatur, in der Premier Howejdā elf israelische Fußballspieler mit Augenklappe - eine Anspielung auf den amtierenden israelischen Außenminister Moshe Dayan - aufforderte, das Spielfeld zu verlassen, da das Spiel zu Ende sei. Die israelischen Spieler erwiderten daraufhin, dass sie nicht darin erprobt seien, besetzte Gebiete zu verlassen: „Fragen Sie die Araber!“.

${ }^{5}$ Vgl. auch CHEHABI, Football in Iran, S. $384 \mathrm{ff}$.

${ }^{6}$ CIA Memo, ONE, 8.10.71, encl. to Huizenga Memo, 8.10.71, CREST: \#RDP79*014-1; vgl. CIA Special Memo, 7.5.68, DDRS: CK3100219600.
} 
an das verarmte, geteilte und von ausländischen Mächten dominierte Land, das er bei seiner Thronbesteigung 1941 vorgefunden habe. Iran könne seit Mitte der 1960er Jahre Wachstumsraten von über $10 \%$ vorweisen. 1971 werde das pro-Kopf-Einkommen 350 USDollar erreichen. Zehn Jahre nach Beginn der Landreform hätten sich Gesellschafts- und Besitzstrukturen auf dem Land grundlegend gewandelt. Die Bildungsoffensive käme der breiten Masse der Bevölkerung zugute. Zwar gebe es auch übermäßig brutale Repression der Sicherheitsorgane und die Wahlen seien nach wie vor eine Farce. Aber dennoch werde das Land im Großen und Ganzen ordentlich regiert - gut ausgebildete Technokraten seien dabei, eben jene sozialen und wirtschaftlichen Reformen umzusetzen, die den Kern der oppositionellen Forderungen der 1950er Jahre ausgemacht hatten. Den Erfolg verdanke das Land glücklichen Umständen, enorm gestiegenen Öleinnahmen, generösen Krediten aus dem Ausland, aber vor allem auch dem Regierungsgeschick Mohammad Rezās. Er selbst stehe mehr als noch zuvor im Zentrum des Entscheidungsprozesses, interessiere sich für und entscheide über Details der Landreform, über Bildungsfragen, über die Ölförderung und industrielle Entwicklung. Er plane und bestimme maßgeblich den Ausbau des Militärapparates und der Sicherheitskräfte und diktiere quasi im Alleingang die außenpolitische Orientierung. Mohammad Rezā sei zu einem ,confident ruler“ geworden: „He is sure that his way is best for Iran and that monarchical power, wisely used, is essential to the country's well being. He is, all in all, a popular and respected king."

Das bilaterale Verhältnis zu den USA war weiterhin geprägt durch die Entwicklungen im Zuge der Transformation der beiderseitigen Beziehungen nach 1963. Anfang der 1970er Jahre war vom früheren Klientel-Verhältnis kaum noch etwas übriggeblieben. Die für die Beziehungen während der 1960er Jahre so grundlegenden Fragen der Militärpolitik blieben auch in der Folgezeit von herausragender Bedeutung. Im Zuge der Ablösung des amerikanischen Militärmonopols 1966/67 - wie in Kapitel VI oben ausgeführt - hatte es bereits diverse Anzeichen für eine Fortsetzung der beschleunigten Aufrüstungspolitik seitens Mohammad Rezās gegeben. Das MOU vom Juli 1964 erweiterte das bereits 1962 ausgehandelte FünfjahresMAP-Programm von \$300 Millionen um etwa \$330 zusätzliche Millionen, vorwiegend auf Kreditbasis. Die Ergänzung zum MOU vom August 1966 hatte sodann die Gesamtsumme für US-Militärgüter bis 1970 auf etwa $\$ 850$ Millionen hochgeschraubt. Dazu kamen noch Waffengeschäfte mit dritten Staaten, unter anderem die Vereinbarung mit der UdSSR vom Januar 1967 über Käufe in Höhe von \$90 Millionen. Insgesamt hatte der Schah bereits bis 1967 zukünftige Waffenkäufe in Höhe von über $\$ 1$ Milliarde vereinbart. ${ }^{8}$

\footnotetext{
${ }^{7}$ Ebd., S. 6; vgl. State \#198765, 19.5.67, FRUS 64-68, XXII, S. 371f.

${ }^{8}$ Vgl. State Background Paper,U.S. Military Assistance to Iran', 15.8.67, ebd., S. 402ff.; Saunders Memo, 17.5.67, ,Iran Mil[...]31/67 [1 of 2 $]^{\star}$, NSF, FHHS, Box 15, LBJL; offenbar hatte ARMISH/MAAG dieses Mal den Schah in seinen Wünschen zuerst bestärkt, arbeitete dann aber mit dem State Department zusammen, um das angestrebte Aufrüstungsprogramm wieder zu beschneiden. Vgl. Meyer Letter, 28.12.67, DEF 12-5, CF 67-69; Meyer warnte fortwährend vor den Folgen eines Verlusts der US-Position als ,principal supplier“. Vgl. Tehran \#557, 3.8.67, ,Iran“, Box 473, Harriman Papers, LOC.
} 
Angesichts der Vorgeschichte war es allerdings wenig überraschend, dass der Appetit Mohammad Rezās nach weiteren Rüstungsgütern durch dieses Programm noch nicht gestillt war. Bereits im November 1967 kündigte er in einem Schreiben an Präsident Johnson einen neuen Fünfjahresplan für die iranischen Streitkräfte an. Anvisiert wurden zusätzliche Rüstungskäufe in Höhe von weiteren $\$ 800$ Millionen. Zwar wusste man auf amerikanischer Seite, dass es im Grunde keine militärische Rechtfertigung für eine derart exorbitante Ausweitung der militärischen Kapazitäten gab. Zugleich aber war man sich der Konsequenzen für das bilaterale Verhältnis bewusst für den Fall, man käme den iranischen Wünschen nicht entgegen. ${ }^{9}$ Meyer warnte aus Teheran: ,[... $]$ it is worth noting that in $\{a ; R . P$.$\} world$ increasingly unfriendly to USG $\{$ U.S. government $\}$, Iran's friendship is worth keeping. "10 Wie auch in den Jahren gewährte Washington dem Schah zusätzliche Aufrüstung - der zugesagte Militärkredit für das Finanzjahr 1968 wurde verdoppelt gemeinsam mit einer, wenn auch vorerst formell nicht verbindlichen Zusage über weitere $\$ 500$ Millionen bis $1973 .{ }^{11}$ Mit der Bekanntgabe des britischen Abzugs aus dem Persischen Golf, anberaumt für Ende 1971, hatte der Schah alsbald einen neuen Vorwand gefunden, das Rüstungsprogramm auszubauen. Nun drängte er auf beschleunigte Umsetzung des gerade erst vereinbarten Programms bis 1971, vorgeblich um für das entstehende Machtvakuum im Golf gewappnet zu sein. ${ }^{12}$

Die Nixon-Administration setzte die geerbte Militärpolitik gegenüber Iran kontinuierlich fort. Die Rüstungsverkäufe auf Kreditbasis seien, so eine interne Bestandsaufnahme im April 1969, der „Prüfstein“ der amerikanischen special relationship mit Iran. Dementsprechend hielt man an der heraufgesetzten jährlichen \$100-Millionen-Kreditgewährung fest und entsprach dem Wunsch des Schahs nach Lieferung zweier zusätzlicher $F$-4-Staffeln. ${ }^{13}$ Entsprechend ermutigt, weitete Mohammad Rezā seine Wünsche in gewohnter Manier alsbald aus. Während seines Staatsbesuchs in den USA im Oktober kündigte der Schah die fortgesetzte Expansion seiner Streitkräfte an, unter anderem die Verdoppelung der $F$-4-Staffeln auf nunmehr acht. Zudem müsse man bereits jetzt an ein Nachfolgesystem für die bald veralteten F-5 im iranischen Arsenal für die Zeit nach 1975 denken. Einige Monate später drängte er wieder auf eine Ausweitung der 1968 informell vereinbarten Militärkreditobergrenze auf die bereits zuvor

\footnotetext{
${ }^{9}$ Vgl. Tehran \#2313/\#3708, 28.11.67/11.3.68, DEF 19-8 US-IRAN, CF 67-69; Rostow Memo, 19.12.67, ,Iran, 4/1/[...]2]', NSF, FHHS, Box 15, LBJL; Shah Letter, 15.11.67; Saunders Memo, 22.3.68, FRUS 64-68, XXII, S. 436ff., $486 f$.

${ }^{10}$ Tehran \#3869, 23.3.68, ebd., S. 490; Meyer versuchte Bedenken zu zerstreuen, zusätzliche Waffen könnten einen Rüstungswettlauf im Golf auslösen bzw. den Schah zu offensiven Aktionen gegen die arabischen Nachbarn verleiten: „We still can cut off arms supplies, as we did \{sic\} Pakistan, if Shah misbehaves in Gulf area.“; ebd., S. 488.

${ }^{11}$ Vgl. Rostow Memo, 29.4.68; State \#166827, 18.5.68, ebd., S. 507ff., 510ff.; vgl. Reports of the Political Study Group, o.D., encl. to Read Memo, 7.11.68, DEF 19-8 US-IRAN, CF 67-69; somit verdoppelte sich de facto die jährliche Militärkreditrate ab diesem Zeitpunkt. Vgl. MCGLINCHEY, Arms Credit Sales, S. 242-6.

${ }^{12}$ Vgl. Tehran \#7433, 24.11.68, FRUS 64-68, XXII, S. 564ff.

13 Vgl. NSC Interdepartmental Group for Near East and South Asia Meeting, 3.4.69; Kissinger Memo, 29.4.69, FRUS 69-76, E-4, docs. 10, 13; zur generellen Praxis der Nixon-Administration bez. Militärhilfe vgl. HuNT, History of the Office of the Secretary of Defense: VII, S. 463-92.
} 
geforderten $\$ 800$ Millionen. Angesichts des impliziten Finanzbedarfs ergab sich nun eine von US-Seite kaum erwünschte enge Verbindung zwischen Aufrüstungsprogramm und Öleinnahmen. Ohne Entgegenkommen der US-Administration hinsichtlich der Militärkredite und der gewährten Konditionen bezüglich Laufzeit und Zinssätzen verstärkte sich zwangsläufig der Druck des Schahs auf das Ölkonsortium, um die Einnahmesituation zu verbessern. Mohammad Rezā versuchte sogar zeitweilig, eine direkte Verbindung zwischen beiden Feldern herzustellen, indem er ein spezielles Arrangement vorschlug, das den Direktverkauf iranischen Öls in den USA und die Verwendung der resultierenden Einnahmen für Rüstungskäufe vorsah. ${ }^{14}$

Die Dynamiken ähnelten jenen der Johnson-Ära. Obgleich große Zweifel bestanden hinsichtlich der militärischen Begründungen der Iraner zugunsten eines weiteren Aufrüstungsschubes, der Vereinbarkeit mit der fortgesetzten wirtschaftlichen Entwicklung des Landes und der Fähigkeit der Streitkräfte, zusätzliche Waffensysteme zu absorbieren, beugte sich letztlich auch die Nixon-Administration dem Druck Mohammad Rezās. Wieder einmal drohte er mit einer Krise in den bilateralen Beziehungen und nach hergebrachtem Muster mit weiteren Rüstungsgeschäften mit Moskau bzw. der Umorientierung seiner Beschaffung in Richtung europäische Lieferanten. Amerikanische militärische Experten zweifelten an der Fähigkeit der Iraner, ein solches Ausmaß an modernem Waffenmaterial überhaupt unterhalten zu können. Bereits zu diesem Zeitpunkt beanspruchten die Iraner die Hälfte aller Ausbildungsplätze, die in den USA für ausländische Piloten insgesamt zur Verfügung standen. Ein weiteres kaum zu lösendes Problem war das Fehlen gut ausgebildeter Fluggerät-Techniker auf iranischer Seite, die für den Unterhalt der technologisch hochkomplexen Kampfflugzeuge unabdingbar waren. ${ }^{15}$,The Shah desires to purchase amounts of military equipment that exceed the ability of his services to absorb, lie far beyond his military requirements, and run the risk of destabilizing the military balance in the Persian Gulf area", warnte das Pentagon im Oktober

\footnotetext{
${ }^{14}$ Vgl. MemCon, 22.10.69; Tehran \#1019, 19.3.70, FRUS 69-76, E-4, docs. 36, 55; der iranische Vorschlag zielte auf die Lieferung iranischen Öls für den Aufbau einer amerikanischen strategischen Ölreserve, gelagert in Salzkammern bzw. alten Minen. Bei Lieferung würde der Produktionspreis fällig und erst bei Verwendung der Reserve der Aufschlag bis zum aktuellen Marktpreis: „Essentially the Iranians are trying to create a situation in which - at no cost to Iran - any oil coming into the US in excess of normal imports would come from Iran."; Saunders Memo, 11.9.69, ebd., doc. 20; angesichts der eingeleiteten internen Debatten zur Revision der Ölimportquoten wurden die Iraner anfangs vertröstet. Nixon erteilte der Idee sodann im Juli 1970 eine Absage. vgl. Saunders Memo, 5.11.69; Flanigan Memo, 10.1.70; Nixon Letter, 30.7.70, ebd., docs. 38, 44, 80; offenbar ging die ursprüngliche Idee auf das erfolgreiche Lobbying des US-Konsortiums Planet Oil and Mineral zurück. Vgl. Mollenhoff Memo, 17.10.69, ,Visit [...]23 1969, 1 of 2', NSCF, VIP Visits, Box 920, RMNL; Nixon Letter, 16.4.70, DDRS: CK3100726075; einige Jahre und Ölpreiserhöhungen später erinnerte sich Mohammad Rezā an seine damals abgelehnte Offerte und kommentierte sarkastisch „I will never make you that offer again.“; Tehran \#2166, 7.4.73, FRUS 69-76, XXVII, S. 41.

${ }^{15}$ Vgl. Tehran \#1247, 1.4.70, FRUS 69-76, E-4, doc. 57; zu diesem Zeitpunkt warnte MacArthur II noch gemeinsam mit dem Kommandeur von ARMISH/MAAG, Generalmajor Hamilton Twitchell, davor, weitere $F$-4Staffeln zu ordern, da man nicht in der Lage sei, diese in die Luftwaffe zu integrieren. MacArthur II plädierte dennoch für eine informelle Zusage über die Weiterführung der Vereinbarung von 1968 um 3-4 weitere Jahre. Vgl. MemCon, 14.4.70, ebd., doc. 61; vgl. Tehran \#4215, 15.10.69; Sisco Memo, 18.10.69, POL 7 IRAN, CF 6769; Tehran \#1247, 1.4.70, ,Iran, Vol. I, [...]70‘, NSCF, Country Files-Middle East, Box 601, RMNL.
} 
1970. ${ }^{16}$ Das Weiße Haus im Verbund mit der Botschaft und der CIA setzte sich dennoch gegen den Widerstand von Pentagon und Teilen des State Department durch - wieder einmal wurden die Wünsche des Schahs weitgehend erfüllt und die zugesagte Anzahl zusätzlicher F-4Kampfflugzeuge wurde in der Tat verdoppelt. ${ }^{17}$ Botschafter Douglas MacArthur II verteidigte später seine Haltung. Er sei keinesfalls für die Gewährung eines „Blankoschecks“ an Mohammad Rezā, aber der fortgesetzte US-Einfluss im militärischen Bereich sei eben nur bei Entgegenkommen gegenüber den Wünschen des Schahs weiter gewährleistet. ${ }^{18}$ Kaum waren ihm die Lieferung zusätzlicher F-4-Staffeln zugesichert, konzentrierte sich der Schah auf den Erwerb der nächsten Generation von Kampfflugzeugen. Nun drängte er auf Lieferung von drei F-15-Staffeln bis 1975, also zeitgleich mit der geplanten Einführung dieses sich noch in der Entwicklung befindlichen, hochmodernen Kampfflugzeugs in der US-Luftwaffe. Zudem forderte er wiederholt ab Januar 1972 eine zusätzliche Beschleunigung der Lieferung der neuen F-4- und F-5E Tiger II-Staffeln. ${ }^{19}$ Die kaum noch zu beherrschende Ausweitung iranischer Beschaffungswünsche und der sofort einsetzende Druck, einmal zugesagte Lieferungen zu beschleunigen, illustrierten die Zweifelhaftigkeit der Beteuerungen MacArthurs II und anderer US-Offizieller, man verfüge über nachweisbaren Einfluss auf die militärischen Planungen des Verbündeten. INR im State Department - eine der wenigen verbliebenen Bastionen einer kritischen Haltung zum Schah innerhalb der Administration - betonte dementsprechend, Mohammad Rezā sehe in ARMISH/MAAG inzwischen nicht viel mehr als eine Serviceagentur zur Durchführung von Rüstungskäufen. Tatsächlicher Einfluss auf Ausmaß und Richtung der iranischen Rüstungsanstrengungen sei in der Realität nicht auszumachen. ${ }^{20}$

\footnotetext{
${ }^{16}$ Nutter Memo, 12.10.70, FRUS 69-76, E-4, doc. 90; Laird Letter, 27.10.70, DEF 12-5 IRAN, CF 70-73; Armin Meyer behauptete später die Existenz einer Twitchell Doctrine, benannt nach dem ehemaligen ARMISH/MAAGLeiter: „General Twitchell thought that Iran's procurement policies should be very carefully and closely coordinated with its training programs, so that purchase equipment would be absorbed and absorbable when it arrived on the scene.“; AMIRIE/TwITCHELL, Iran in the 1980s, S. 379f.; die „Doktrin“ sei anschließend auch in Richtung auf ökonomische Tagfähigkeit neuer Erwerbungen ausgedehnt worden. Für bare Münze genommen und die Entwicklungen durchgehend fehleinschätzend in COOPER, Oil Kings, S. 23f., 59; in den Quellen findet sich kein Beleg für eine solche Doktrin, die wohl eher der ex post facto-Legendenbildung nach der Revolution zuzuschreiben ist. Tatsächlich trat Twitchell, gegen starke Widerstände des Pentagons und trotz erheblicher rechtlicher Bedenken, im Anschluss an seine Tätigkeit als ziviler Berater in den Dienst des Schahs über, offenkundig in erster Linie pekuniär motiviert und im Anschluss offenbar alles andere als mäßigend auf iranische Waffenkäufe einwirkend. Vgl. Tehran \#3589, 3.7.71; Johnson Memo, 8.10.71, FRUS 69-76, E-4, docs. 135, 147; vgl. auch Hamilton A. Twitchell OHI, 5.7.84, DOD FOIA Reading Room.

${ }_{17}$ Das Pentagon akzeptierte letztlich auch die Zusage einer siebten und achten $F$-4-Staffel für Iran. Damit hätte sich das Luftwaffenarsenal bis Ende 1974 auf 132 F-4- und 112 F-5-Kampfflugzeuge erhöht. Vgl. Kissinger Memo, 16.4.70; Saunders Memo, 2.9.70; State \#174651, 23.10.70; Laird Letter, 27.10.70; Saunders Memo, 4.11.70; State \#183657, 6.11.70; Nutter Memo, 7.12.70, FRUS 69-76, E-4, docs. 62, 85, 92, 93, 94, 96, 103; Tehran \#3387, 7.8.70, DEF 12-5 IRAN; MemCon, 14.4.70, DEF 19-8 US-IRAN, CF 70-73; Saunders Memo, 15.12.70, ,Iran, Vol. II, [...]70‘, NSCF, Country Files-Middle East, Box 601, RMNL; Eliot Jr. Memo, 27.3.70, , NSC/Misc-March 1970‘, MLR Entry A1 5002, General Files on National Security Council (NSC) Matters, 1/1969-12/1972, Box 16, RG 59, NACP; vgl. ALVANDI, Origins of Primacy, S. 353-6.

${ }^{18} \mathrm{Vgl}$. MemCon, 8.4.71, FRUS 69-76, E-4, doc. 123.

${ }^{19}$ Vgl. Tehran \#7283, 22.12.71, ebd., doc. 154; vgl. MemCon, 18.1.72, encl. to Touissant Letter, 22.1.72, DEF 198 US-IRAN, CF 63; das Dokument sollte eigentlich in den Central Files für 1970-73 abgelegt sein. Vgl. Tehran \#2890, 17.5.72, FRUS 69-76, E-4, doc. 193; zur Rettung des Rüstungskonzerns Grumman als „Nebeneffekt“ der F-14-Order vgl. BILL, Eagle and Lion, S. 201f.; vgl. auch TelCons, 28.7.72 und 29.7.72, DNSA:HAKTelCons: \#KA08441 und \#KA08453.

${ }^{20}$ INR Research Study RNAS-2: ,Iran: Arms and the Shah', 28.1.72, FRUS 69-76, E-4, doc. 164.
} 
Trotz dieser vereinzelten Zweifel an der eigenen Strategie hinsichtlich der iranischen Aufrüstung und ihren möglichen langfristigen Folgen etablierte sich 1972 eine neue amerikanische Position in dieser Frage, welche die im Kern seit 1962 zumindest formell bestehende Handhabung von Waffenlieferungen an Iran durch die USA im Grundsatz revidierte. Kontext der Entscheidung war der seit langer Zeit vom Schah gewünschte Iranbesuch eines amerikanischen Präsidenten seit Eisenhowers Kurzvisite von 1959: Ende Mai 1972 trafen Präsident Richard M. Nixon und sein Nationaler Sicherheitsberater Henry Kissinger auf der Rückreise vom Gipfel in Moskau in Teheran ein. Mohammad Rezā trug Sorge, dass dem ihm seit zwei Jahrzehnten zugetane Nixon ein wahrhaft königlicher Empfang in Iran zuteil wurde. Richard Nixon und die First Lady logierten im Sa d-Ābād-Palast, die Fahrt durch die Straßen Teherans glich einem Triumphzug. Hunderttausende säumten den Weg, perfekt durchorganisiert auf Anordnung von Hofminister Asadollah 'Alam. ${ }^{21}$ In den Gesprächen in jenen Tagen erfüllten sich gewissermaßen die langgehegten Träume Mohammad Rezās für die Stellung Irans in der amerikanischen globalen Gesamtstrategie. Die offenkundige Analogie war die sogenannte Nixon-Doktrin - eine letztlich vage und nie ganz geklärte Wende in der US-Politik gegenüber den Staaten der Peripherie, die häufig als Rückzug der USA in die Position eines Offshore Balancer fehlinterpretiert wurde. Im Kern zielte die Doktrin auf eine Vermeidung einer Involvierung in Vietnam-ähnliche Szenarien in der Zukunft, also die Ablehnung direkter militärischer Verwicklung in interne Konflikte an der asiatischen Peripherie. Bald umgedeutet in eine globale Grand Strategy, verband man später mit der Nixon-Doktrin die Übertragung der bisher selbst ausgeübten Stabilisierungspolitik in Kernregionen an örtliche Surrogatmächte, die diplomatisch und wirtschaftlich unterstützt durch die USA, aber auch militärisch aufgerüstet, nunmehr gewissermaßen im Auftrag Washingtons ordnungspolitisch tätig werden sollten. ${ }^{22}$ Im Vorfeld des Teheranbesuchs erläuterte Sicherheitsberater Kissinger das Ziel der US-Politik, freundlich gesinnte Regionalmächte darin zu unterstützen, ,[...] to develop the capacity to do what the US can no longer do around the world in providing the principal ingredients of regional security and stability." 23 Analog begründete Präsident Nixon seine Zusage an Mohammad Rezā in den Teheraner Gesprächen, den weitreichenden Rüstungswünschen Irans entgegenzukommen, mit der ausdrücklichen Aufforderung „Protect me“. 24

Mohammad Rezā hatte diesen Wandel in der amerikanischen Herangehensweise bereits früh

${ }^{21}$ Vgl. die Schilderung des Besuchs bei CoOPER, Oil Kings, S. 58f., vgl. auch WAWro, Quicksand, S. 297ff., 3326.

${ }_{22}$ Vgl. die kritische Einschätzung bei KImBall, Nixon Doctrine; HANHIMÄKI, Flawed Architect, S. 53f.; die Bewertung der Grand Strategy der Nixon-Administration als eher herkömmliche und vorwiegend taktische Anpassung bei ders., Elusive Grand Design.

${ }^{23}$ Kissinger Memo, 18.5.72, FRUS 69-76, E-4, doc. 196; in demselben Memorandum legt Kissinger im Detail die Anwendung der Nixon-Doktrin auf Iran dar, u.a. mit Bezug auf die künftige Ordnung im Golf: „In the Persian Gulf, the US does not have any aspirations to assume the British role. This is an area where a new kind of US presence must evolve; in substantial part it will be provided by American business, technological, educational and diplomatic relationships."; ebd.

${ }^{24}$ MemCon, 31.5.72, ebd., doc. 201; vgl. Einträge vom 29.5. und 1.6.72, in: ALAM, Shah and I, S. 221-6. 
antizipiert und für die eigenen Zwecke zu instrumentalisieren gesucht. ${ }^{25}$ Bereits im Vorfeld seines Washingtonbesuchs im Juli 1967 hatte der Schah einen weiteren Ausbau seines militärischen Apparates gefordert, ,[...] so that if troubles break out in this region American boys will not be shot a la Viet Nam.“26 Angepasst an die Rhetorik der neuen NixonAdministration verwies Mohammad Rezā im März 1969 ein weiteres Mal auf die Möglichkeit „,zukünftige Vietnams“ zu verhindern, indem man auf „self-reliant and progressive friends like Iran" setze, ,[...] so that such countries can exercise fruitful responsibility in their respective regions““. ${ }^{27}$ Im Frühling 1970 verteidigte der Schah seine Wünsche nach einer Verdoppelung der zugesagten $F$-4-Staffeln und der erneuten Verletzung der vereinbarten Kreditobergrenzen mit der lapidaren Begründung, er implementiere die Nixon-Doktrin und diese Haltung müsse eben entsprechend gewürdigt werden. INR warnte bereits im Januar 1972, Mohammad Rezā sehe die Nixon-Doktrin in erster Linie als Gelegenheit, seine strategischen Ambitionen im Persischen Golf und im Indischen Ozean zu verwirklichen. Tatsächlich hatten amerikanische Offizielle selbst wiederholt und auch öffentlich die Verbindung zwischen Nixon-Doktrin und Iran hergestellt und den Schah in seinem Glauben bekräftigt, ,chosen instrument“ der USGesamtstrategie im Golf zu sein. ${ }^{28}$ Im Vorfeld des Nixon-Besuchs von 1972 verdichteten sich in den internen Diskussionen die Zeichen, dass von einer Fortsetzung des inkrementellen Entgegenkommens gegenüber den ausufernden iranischen Beschaffungswünschen - im Kern, in Zweijahresabständen, das Grundmuster seit dem MOU von 1964 - Abstand genommen werde. Die CIA verwies zeitgleich auf die Vervierfachung des iranischen Militäretats seit 1966 - Iran habe seit Mitte der 1950er Jahre Waffen im Wert von \$1,8 Milliarden eingeführt und für weitere \$1,4 Milliarden bestellt und sei bereits jetzt zum sechstgrößten Waffenimporteur in der Dritten Welt aufgestiegen. Man rechnete mit einer Stabilisierung auf hohem Niveau, stellte aber sogleich fest, dass ,[...] Tehran should be able to finance the foreign exchange costs of military imports without difficulty from increased oil revenues. “29

\footnotetext{
${ }^{25}$ In den Worten Hanhimäkis: „the Shah himself quickly made the most of the Nixon Doctrine.“; ders., Elusive Grand Design, S. 39.

${ }^{26}$ Tehran \#447, 24.7.67, FRUS 64-68, XXII, S. 396; der Schah hatte über andere Quellen auch von der Einsetzung einer Special State-Defense Study Group im März 1967, unter Leitung des ehemaligen US-Botschafters Julius Holmes erfahren. In einem persönlichen Treffen mit Holmes versuchte der Schah sogleich, die Studie in seinem Sinne zu beeinflussen. Vgl. Holmes Memo, 24.8.67, ebd., S. 424; Aufgabe der Study Group war es, den Charakter und Umfang der langfristigen amerikanischen Interessen in der Großregion Nordafrika-Naher und Mittlerer OstenHorn von Afrika zu untersuchen und Politikempfehlungen abzugeben. Weniger überraschend angesichts von Holmes' Überzeugungen empfahl die Gruppe im Juli 1967 verstärktes US-Engagement angesichts der zunehmenden indirekten sowjetischen Bedrohung in der Region und bedingungslose Unterstützung der zentralen amerikanischen Verbündeten und gerade Irans. Vgl. Report Prepared by the Special State-Defense Study Group, n.d. [July 1967], ,Near East, North Africa and the Horn of Africa', FRUS 64-68, XXI, S. 49-58; intern wurde die Studie und die sie durchziehende, allzu traditionelle Sichtweise allerdings scharf kritisiert. Harold Saunders empfahl ein breites „Disengagement“ statt neuen amerikanischen Aktivismus. Sowjetischem Einflussgewinn seien ähnliche Grenzen gesetzt wie dem westlichen: „We can gamble that nationalism will win out over Communism - that it will reject Moscow's domination and become something we can live with.", Saunders Memo, ,The US and the USSR in the Middle East', 5.9.67, attached to Saunders Memo, ,State, [...](IRG), [2 of 2]', NSF, Agency File, Box 63, LBJL; letztlich scheiterte die seit Ende 1966 angestrebte Ausformulierung einer umfassenden Nahoststrategie der USA an eben diesen Gegensätzen. Vgl. Critchfield Memo, 8.1.68; Earle/Orwat Memo, 22.11.68, FRUS 64-68, XXI, S. 68ff., 79ff.

${ }^{27}$ Vgl. FRUS 69-76, E-4, doc. $7 n 1$.

${ }^{28}$ Vgl. Tehran \#1019/\#1247/\#3144, 19.3./1.4./ 22.7.70; INR Research Study RNAS-2, 28.1.72, ebd., docs. 55, 57, 78, 164; vgl. ALVANDI, Origins of Primacy, S. 356.

${ }^{29}$ Vgl. CIA Memo ER IM 72-79, Mai 1972, FRUS 69-76, E-4, doc. 181.
} 
Die Quellen demonstrieren, dass im Jahr 1972 der traditionelle (Rest-) Widerstand innerhalb der US-Administration gegen umfangreiche Waffenverkäufe an Iran nicht mehr gegeben war. Zwar akzeptierte das DOD auch vor dem Nixon-Besuch in tradierter Manier nicht alle ausufernden Forderungen Mohammad Rezās. Begründet wurde die Verweigerung prinzipieller Zusagen aber nun nicht mehr mit den möglichen Folgen für die interne wirtschaftliche und finanzielle Balance Irans im Sinne der alten Annual Review oder möglicher geopolitischer Konsequenzen, sondern nunmehr in erster Linie mit der Priorität der eigenen Bedürfnisse bzw. der fehlenden Planungssicherheit aufgrund von Mohammad Rezās Interesse an Waffensystemen, die sich noch in der Entwicklung befanden und deren Erstauslieferung und Produktionsgeschwindigkeit noch nicht seriös vorauszusagen waren. Tatsächlich stimmte das DOD bereits vor der Teheranvisite Nixons und Kissingers im Grundsatz dem Verkauf neuartiger lasergelenkter Bomben, der Luft-Boden-Rakete Maverick, der Aufstockung der iranischen F-5E- und F-4-Staffeln auf jeweils zehn und der Zusage der Entsendung zusätzlicher amerikanischer Techniker zu. Allein dem exzentrischen Wunsch Mohammad Rezās nach bindenden Zusagen für die Lieferung von F-14- und F-15-Kampfjets - angedacht als Rückgrat der US-Luftwaffe in der Zukunft - wurde nicht entsprochen. Aber selbst hier stellte man in Aussicht, ,[...] that we anticipate favorable action on the sale but the matter must be held in abeyance until the programs become more stable and predictable. ${ }^{\text {(30 }}$ Entgegen der in der Literatur weiterhin dominierenden Interpretation eines Alleingangs Nixons und Kissingers in Teheran und der Gewährung eines seitens der Regierungsbürokratie abgelehnten „Blankoschecks“ muss somit festgestellt werden, dass sich der US-Präsident in seinen Zusagen an Mohammad Rezā zumindest formell im Rahmen der Vorgaben der Departemente befand, wobei er durchaus einige spezifische Details und Caveate in diesen Vorgaben ignorierte.

Der Mythos des „Blankoschecks“ entstand bereits in den 1970er Jahren im Kontext der kritischen Untersuchung der US-Waffenexporte durch den Kongress und wurde später vermeintlich belegt durch die Veröffentlichung zweier Kissinger-Memoranden durch die Botschaftsbesetzer nach der Revolution. ${ }^{31}$ Insbesondere Kissingers in dieser Schärfe doch

\footnotetext{
${ }^{30}$ Rush Memo, 18.5.72, ebd., doc. 195; die Ausdehnung der F-4 und F-5E-Flotten auf jeweils zehn ist etwas irreführend. Tatsächlich hatte der Schah bereits 1971 eine Modernisierung der überholten $F$ - $5 A$-Flotte und den Ersatz durch die neueren $F-5 E$ angeordnet und insgesamt acht Staffeln bei Northrop bestellt.

${ }^{31}$ Vgl. Kissinger Memos, 15.6./ 25.7.72, Asnād 8, S. 42ff.; beide Memoranden sind inzwischen auch ,regulär“ freigegeben. Siehe FRUS 69-76, E-4, docs. 205, 214; vgl. Saunders Memos, 14./18.7.72, , Iran, Vol. IV, 1 Sep 71Apr 73', NSCF, Country Files-Middle East, Box 602, RMNL; das Pentagon hatte im Falle der $F-14 / F-15$-Anfragen und der lasergelenkten Bomben zu einem Vermeiden konkreter Zusagen und Liefertermine geraten. Nixons Selbstverpflichtung ging in beiden Fällen etwas weiter als vom Pentagon intendiert. Angesichts der klaren Quellenlage verwundert dennoch die Einschätzung von MCGLINCHEY, Road to Tehran, S. 855, wonach das Pentagon,,wished to effectively retain the restrictionist approach to Iran, allowing him a limited number of replacement jets, yet effectively denying the shah a qualitative upgrade in his fleet."; vgl. dagegen State Briefing Book, Mai 1972, DNSA: IR00773; die Kongress-Untersuchung aus dem Jahr 1976 befand, der Entscheid von 1972 habe „Iran effectively exempted from arms sales review process in State und DOD.“; im Anschluss sei jede interne Kontrolle de facto weggefallen: ,, for at least three years U.S. arms sales to Iran were out of control and the programs
} 
ungewöhnliche Formulierung, der Präsident vertrete den Standpunkt, „,...] that, in general, decisions on the acquisition of military equipment should be left primarily to the government of Iran“, blieb im kollektiven Gedächtnis als freiwillige Aufgabe jeglicher Steuerungsfunktion seitens der USA haften. ${ }^{32}$ William Bundy, der Bruder des ehemaligen Sicherheitsberaters McGeorge Bundy und selbst ehemaliger Offizieller in den demokratischen VorgängerAdministrationen, bezichtigte Nixon später, jede Debatte über diese politische Wende unterbunden zu haben , ,...] by deciding simply that the Shah should get what he wanted.“33 Im Anschluss an die Revolution und den Abbruch der bilateralen Beziehungen ging die Forschungsliteratur dazu über, den sogenannten „Blankoscheck“ vom Mai 1972 als grundsätzliche Wende in den amerikanisch-iranischen Militärbeziehungen zu interpretieren. Der ehemalige Under Secretary of State George Ball kritisierte, die Gewährung von ,[...] free access to the whole range of advanced items in our military arsenal was like giving the keys of the world's largest liquor store to a confirmed alcoholic. “34 Entgegen der umsichtigen und retardierenden Handhabung iranischer Aufrüstungswünsche in der vorhergehenden Dekade so die spätere Deutung - habe der Richtungswechsel vom Frühling 1972 alle Schleusen geöffnet und die geradezu wahnwitzigen Waffenkäufe Mohammad Rezās mit all ihren nachteiligen Folgen für die wirtschaftliche Balance Irans und für seine eigene Legitimität gegenüber der eigenen Bevölkerung erst möglich gemacht. Der „Blankoscheck“ wird somit als eines der Schlüsselereignisse mit Bezug auf die spätere revolutionäre Umwälzung und insbesondere die sich parallel konstituierende Feindschaft zwischen den beiden ehemaligen Verbündeten angesehen. ${ }^{35}$ Wenig überraschend interpretierte Mohammad Rezā selbst die Zusagen Nixons in Teheran ebenfalls als Versprechen, in Zukunft ohne Einschränkungen ,[...]

were poorly managed.“; SenAte Subcommittee, Sales to Iran, S. VIII, XII; zitiert in BILL, Eagle and Lion, S. 200.

${ }^{32}$ Kissinger Memo, 25.7.72, FRUS 69-76, E-4, doc. 214; George Ball beschwerte sich 1979 gegenüber Arthur Schlesinger, dass eben dieser Entscheid der Startpunkt der späteren Schwierigkeiten gewesen sei. Es sei kein "failure of intelligence“, sondern ein „failure of policy“ gewesen. Zudem beschwerte sich Ball, die NixonAdministration habe die Direktive aus den Akten entfernt (,,rather shocking this“), die er sich daher erst aus den Nixon Papers habe besorgen müssen. SCHLESINGER, JR., Journals, S. 458.

33 W. BuNDY, Tangled Web, S. 329.

${ }^{34}$ BALl, Another Pattern, S. 455; Ball behauptet, die Johnson-Administration habe dagegen ,held firm“ gegen die iranischen Forderungen nach Waffen: ,When he threatened to turn to the Soviet Union for help, we told him to go ahead. [...] we held our position so firmly that during the entire period of nineteen years from his return to power in 1953 to 1972 , his total arms purchases from the United States amounted to only $\$ 1.2$ billion."; ebd., S. 454; der ehemalige ARMISH/MAAG Leiter beschrieb das Verhalten des Schahs im Hinblick auf Waffenkäufe als ,just like a kid in a candy store, and every time he saw something that looked pretty, he wanted some for his country."; Ellis Williamson OHI, 13.4.88, S. III-175.

${ }^{35}$ Vgl. die Einordnung bei BILL, Eagle and Lion, S. 200ff., CotTAM, Iran and the United States, S. 148ff., Pollack, Persian Puzzle, S. 103ff.; RUBIN, Good Intentions, S. 261, nennt den „Blankoscheck“ ,shortsighted and almost criminally careless"; ähnlich HovEYDA, Fall of the Shah, S. 77: „It is safe to say that most of the subsequent difficulties of the regime date from that day."; PETERSEN, Making Allies, S. 88, glaubt, dass der NixonAdministration selbst das Ausmaß des eigenen Zugeständnisses nicht bewusst gewesen sei. PALMER, Guardians, S. 88, zufolge habe die Umsetzung der Nixon-Doktrin im Persischen Golf einen seit 25 Jahren bestehenden Grundsatz amerikanischer Strategie revidiert, wonach außer dem NATO-Verbündeten Türkei kein nahöstlicher Staat massiv aufzurüsten sei: ,The United States had been rejecting the shah's schemes to build up the Iranian military since 1946."; dies ist weder für den iranischen Fall ab 1964 noch für Israel wirklich zutreffend. ALVANDI, Origins of Primacy, S. 361, 369ff., sieht den „Blankoscheck“ als Umsetzung einer neuen strategischen Ausrichtung gegenüber der Golfregion durch die USA. Mit der Aufhebung sämtlicher Restriktionen bez. Waffenverkäufen an Teheran sei eine Akzeptanz und Unterstützung eines iranischen Primats in der Region einhergegangen. MCGLINCHEY, Road to Tehran, S. 841, sieht den „Blankoscheck“ als ,highly irregular move, and a revolutionary change in both general U.S. arms sales policy and in U.S.-Iran relations“. 
all available sophisticated weapons short of the atomic bomb“ zu liefern. ${ }^{36}$ Und es ist in der Tat nicht $\mathrm{zu}$ bestreiten, dass Nixon und Kissinger in ihren internen Weisungen an die amerikanische Regierungsbürokratie darauf drängten, die bisherige Praxis in der Behandlung iranischer Rüstungswünsche im Grundsatz zu ändern. ${ }^{37}$

Eine Betrachtung der Entscheidungsfindung innerhalb der Administration selbst und die Einbettung in den historischen Kontext zeigen jedoch, dass der so häufig konstatierte Wendepunkt vom Mai 1972 kaum als solcher zu bewerten ist. Weder gab es, wie so häufig behauptet, einen Alleingang Nixons und Kissingers in Teheran, noch waren die Folgen der dort getroffenen Zusagen so schwerwiegend, wie die spätere historische Mythenbildung suggerierte. Mit Blick auf die quantitativen Details lässt sich an diesem Punkt zwar durchaus ein grundsätzlicher Wandel konstatieren, was Waffenkäufe und deren Handhabung anging - allein bis Ende 1972 bestellten die Iraner zusätzliche Militärgüter im atemberaubend erscheinenden Gesamtwert von etwa \$2 Milliarden. ${ }^{38}$ Insgesamt aber überwiegen in der Rückschau die Elemente der Kontinuität zu den Vorgängern. Die vorhergehenden Kapitel haben gezeigt, dass spätestens mit dem MOU von 1964 amerikanische Steuerungsfähigkeit iranischer Militärpolitik weitgehend zurückgedrängt worden war. Im Zweijahresrhythmus - 1966, 1968 und 1970 folgten kontinuierlich weitere Grundsatzentscheidungen der Johnson- und NixonAdministrationen, die bereits im Kern immer ein fast vollständiges Entgegenkommen gegenüber den Wünschen Mohammad Rezās implizierten, wenngleich jeweils mit dem Feigenblatt einer vorausgehenden gemeinsamen Prüfung der Vereinbarkeit neuer Rüstungskäufe mit der allgemeinen wirtschaftlichen Entwicklung. ${ }^{39}$ Der enorme Anstieg der Waffenimporte nach 1972 ist zudem wohl nur in Teilen auf eine politisch-strategische Neueinschätzung zurückzuführen - plausibler erscheint es, den rasanten Zuwachs in erster Linie auf die nun sprunghaft steigenden Öleinnahmen zurückzuführen. ${ }^{40}$

\footnotetext{
${ }^{36}$ So der Schah gegenüber ARMISH/MAAG-Leiter Williamson. Siehe CARMISH/MAAG to DOD, encl. to Saunders Memo, 12.6.72, FRUS 69-76, E-4, doc. 204.

${ }_{37}$ Von einer Aufoktroyierung der neuen Politik auf ein widerstrebendes State Department, wie von W. Bundy behauptet, kann jedoch keine Rede sein. In NEA wurde die Direktive offenbar begeistert aufgenommen: ,, a very satisfactory memorandum from Dr. Kissinger which gives everyone his marching orders [...]"; Miklos Letter, 26.7.72, Asnād 8, S. 45; ähnlich Miklos Letter, 20.6.72, ,DEF 19-2A [...]72‘, Lot 75 D 351, NEA/IRN: 1965-75, Box 7.

${ }^{38}$ Vgl Heck Letter, 11.11.72, ,POL 1-[...]72‘, Lot 75 D 351, NEA/IRN: 1965-75, Box 7; State Briefing Paper ,U.S.-Iranian Military Cooperation“, Juli 1973, ,Shah of Iran (17 July 1973)‘, NSCF, VIP Visits, Box 920, RMNL; von 1965 bis 1973 sollten sich die iranischen Verteidigungsausgaben schließlich vervierfachen. Mit den Ölpreiserhöhungen in der ersten Hälfte der 1970er Jahre waren nunmehr weitaus größere Finanzmittel verfügbar. Zwischen 1971 und 1975 verzehnfachten sich die Rüstungsausgaben. Vgl. CHUBIN, Iran's Foreign Policy; zur Entwicklung der Streitkräfte vgl. WARD, Military History of Iran, S. 191-205; auch COTTRELL, Armed Forces.

${ }^{39} \mathrm{Im}$ Grundsatz zuzustimmen ist somit C.D. Carr, der bereits 1981 ohne Zugriff auf Primärquellen feststellte: ,The trauma induced by such crises, coupled with a carefully constructed campaign of diplomatic attrition on US-Iranian security matters emanating from the Shah, so effectively narrowed US options in regard to Iran that by 1972, when President Nixon allowed the Shah unprecedented carte blanche on arms procurement from the United States, this was simply the recognition of the status quo." \{Hervorheb. i. Orig.\}; CARR, Reverse Influence, S. 57; ähnlich William Olson in einer Buchbesprechung in Iranian Studies 19, 3/4 (1986), S. 292f.; die 1970 zugestandene Verdoppelung der F-4-Staffeln für Iran auf insgesamt acht hatte bereits impliziert, dass die 1968 vereinbarte jährliche Kreditobergrenze von \$100 Millionen unmöglich aufrecht zu erhalten war. Vgl. State \#202976, 14.12.70, DEF 12-5 IRAN, CF 70-73; siehe auch Kissinger Memo, 16.4.70, FRUS 69-76, E-4, doc. 62.

${ }^{40}$ Die Einkünfte aus der Erdölförderung waren bereits von \$470 Millionen im Jahr 1964 auf \$854 in 1968 und $\$ 1,14$ Milliarden in 1970 gestiegen. Nach den weitreichenden Zugeständnissen der Ölgesellschaften in 1970 und
} 
Im Allgemeinen hatte sich zudem bereits im Vorjahr innerhalb des DOD ein grundsätzliches Umdenken bezüglich Waffenverkäufen an dritte Staaten angedeutet, und zwar in erster Linie motiviert von ökonomischen Überlegungen. Secretary of Defense Melvin Laird empfahl Nixon bereits im April 1971, die bestehende US-Politik zu Waffenexporten zu lockern, um dem wachsenden europäischen und auch sowjetischen Wettbewerb zu begegnen. Explizit brachte er eine Liberalisierung der bislang einigermaßen strikten Exportkontrollen mit der seitens der Administration propagierten Nixon-Doktrin in Verbindung, die eine verstärkte Zusammenarbeit mit pro-westlichen regionalen Vormächten in der Verfolgung amerikanischer Interessen vorsah. Die diesen zugedachte neue Rolle als Wahrer westlicher strategischer Interessen sei aber nur mit einer entsprechenden Machtposition in der jeweiligen Weltregion umsetzbar, was eine Ausweitung der Waffenlieferungen an diese Schlüsselstaaten geboten erschienen ließ, so Lairds Argumentation. ${ }^{41}$ Tatsächlich hatte sich seit der Ablösung des USMilitärmonopols die Diversifizierung der iranischen Rüstungsbeschaffung beschleunigt. Inzwischen zeichneten sich die USA nur noch für etwa die Hälfte der Waffeneinkäufe Irans verantwortlich, dominierten aber weiterhin den Hochtechnologiebereich, vor allem die Luftwaffe. Großbritannien hatte seine bisherige Sonderstellung bei der iranischen Marine ausgeweitet und lieferte nun auch die nächste Generation von Kampfpanzern für die iranische Armee und wichtige Komponenten der Flugabwehr. Die UdSSR war zum drittgrößten Lieferanten aufgestiegen und hatte im Anschluss an das erste Rüstungsgeschäft von 1967 eine Reihe weiterer Abkommen in Höhe von insgesamt \$370 Millionen mit Iran abgeschlossen. ${ }^{42}$ Somit sind nicht zuletzt ökonomische Motive mit ausschlaggebend für den „Blankoscheck“ gewesen, gerade angesichts der starken Belastungen der amerikanischen Zahlungsbilanz in diesem Zeitraum und der sich aus den rasant gestiegenen Einnahmen der Ölförderländer ergebenden monetären Ungleichgewichte.

1971 erhöhten sich die Einnahmen ein weiteres Mal sprunghaft auf \$1,99 in 1971 und etwa \$2,5 Mrd. in 1972; eine Einschätzung des iranischen Verteidigungsbudgets aus den Akten der CIA zeigt von 1970 bis 1974 einen gleichbleibenden Anteil der Verteidigungsausgaben am Gesamtbudget von in etwa 25\%, in den beiden Folgejahren einen Anstieg zu etwa 35\%. 1977 fiel der Anteil offenbar wieder auf 25\% zurück. Die Gesamtbelastung blieb also in etwa gleich, der kurze Anstieg ist wohl auf die unerwarteten wirtschaftlichen Probleme in der Mitte der Dekade zurückzuführen. Vgl. Schaubild, attached to Notes ,Iran: Roots of Discontent', 25.10.78, CREST: \#RDP81*0698 .

${ }^{41}$ Vgl. Laird Memo, 9.4.71, FRUS 69-76, IV, S. 140ff.

${ }^{42}$ Der US-Anteil am iranischen Waffenmarkt war von nahezu 100\% im Jahr 1966 auf etwa 56\% im Jahr 1972 geschrumpft. Großbritannien verkaufte bis 1972 Waffensysteme im Wert von etwa $\$ 600$ Mio., andere westliche Staaten in Höhe von etwa \$160 Mio. Vgl. CIA Intel Memo \#ER IM 72-79, Mai 1972, FRUS 69-76, E-4, doc. 181; vgl. auch Tehran \#1312, 7.4.70, ebd., doc. 58; der Zusammenhang zwischen Liberalisierung der Ausfuhrbestimmungen und europäischer Konkurrenz wird deutlich in Saunders Memo, 14.7.72, ebd., doc. 212; die Landstreitkräfte erhielten weniger Aufmerksamkeit seitens des Aviatik-Fans Mohammad Rezā, aber auch ihre Modernisierung war in den Staaten der sogenannten Dritten Welt beispiellos. Mit westdeutscher Unterstützung baute Iran nahe Isfahan eine Anlage zur Nachrüstung der fast 900 US-produzierten $M$-47- und $M$ - 60 -Kampfpanzer. 1971 wurden zudem 770 britische Chieftain-Panzer und 250 leichte Scorpion-Spähpanzer bestellt. Vgl. CIA Intel Memo ,Iran: The Shah's Economic and Military Expansion“, Februar 1973; CIA Weekly Review Special Report ,Iran: Building Up the Military', 7.6.74, CREST: \#RDP85*002-3; ein späteres CIA-Dokument nennt sogar sieben Abkommen mit den Sowjets über insgesamt \$480 Millionen. Vgl. CIA Intel Memo ,Iran: The Shah's Economic and Military Expansion', Februar 1973, CREST: \#RDP85*012-2. 
Zuletzt muss noch angemerkt werden, dass der „Blankoscheck“ und die Zusage Präsident Nixons, Iran allein werde in Zukunft über Ausrichtung und Ausmaß des eigenen Aufrüstungsprogramm entscheiden, bei aller nicht $\mathrm{zu}$ bestreitenden Aufweichung des bisherigen Verfahrens dennoch nicht vollständige Handlungsfreiheit für den Schah zur Folge hatten. Iranische Wünsche mussten sich auch im Anschluss mit der generellen Verfügbarkeit der jeweiligen Waffensysteme arrangieren und in der Regel die bevorzugte Auslieferung an die US-Streitkräfte akzeptieren, die sich ja weiterhin in einem Krieg in Indochina befanden. Wenngleich nach Auffassung des Schahs im Mai 1972 jedes Waffensystem mit der Ausnahme von Kernwaffen zugesagt worden war, so ergaben sich in der Praxis aber auch hier eine Reihe von Hindernissen und Verzögerungen, die letztlich doch zu einer - wenn auch erheblich geminderten - fortgesetzten amerikanischen Kontrolle beitrugen. ${ }^{43}$ So konnte sicherheitsrelevante Hochtechnologie ohne entsprechende Freigabe der entsprechenden Gremien nicht exportiert werden. Der interne Widerstand innerhalb der Regierungsbürokratie gegen die Öffnung aller Schleusen war zudem weiterhin präsent. Der zeitgenössische Leiter von ARMISH/MAAG Ellis Williamson bestätigte, auch nach dem „Blankoscheck“ habe es keine quasi automatische Erfüllung der iranischen Wünsche gegeben. Sowohl im DOD wie auch im State Department habe es einflussreiche Gruppen gegeben, die es sich zur Aufgabe gemacht hatten, jede Anfrage möglichst lange zu verzögern. ${ }^{44}$ Auch Kissinger berief sich später in seinen Memoiren auf die weiterhin bestehenden quasi-,,natürlichen“ Hemmnisse und behauptete, Nixons Entscheidung habe sich im Grunde nur auf die Frage der nächsten Generation von Kampflugzeugen bezogen: ,[...] there was no blank check. “45

\footnotetext{
${ }^{43}$ Seitens NEAs wurde in den internen Debatten mit dem DOD der „Blankoscheck“ selbst wieder als Sicherung der US-Kontrolle gegen exzessive Einkäufe der Iraner verteidigt: "It is also important to remember that we do exercise a restraining and rationalizing influence on the Shah and Iranian military development which we would lose - together with the other benefits by our close relations with Iran - were we not to continue in our primary advising/supply role.“; NEA/IRN Briefing Paper, 21.9.72, ,DEF-1 Policy, Plans, Readiness, Iran 1972‘, Lot 75 D 365, NEA/IRN: 1965-75, Box 6; ein Beispiel war die amerikanische Weigerung, Iran U-Boote mit Kernenergieantrieb zu verkaufen. Vgl. TelCon, 8.1.75, DNSA:HAKTelCons: \#KA13128.

${ }_{44}$ Aus dem früheren „Nein“ sei ein „Warte“ geworden. Vgl. Ellis Williamson OHI, 13.4.88, S. III-149f.; ebd., 11.3.88, S. II-78: „There was a large element back in the United States that did not want to give up its power of selection. They just could not accept the fact the Shah was running his own program."; Williamson selbst gab später an, er habe die Lieferung besonders sensibler Waffensysteme verhindert. Vgl. ebd., 10.2.88, S. I-65.; allerdings wurde die Ausfuhr von Systemen wie der schultergestützten Luftabwehrrakete FIM-43 Redeye, ein Vorläufer der Stinger, zugelassen, trotz Bedenken einer möglichen Proliferation zu terroristischen Gruppen und einer möglichen Gefährdung der zivilen Luftfahrt. Vgl. State Department Paper, o.D., FRUS 69-76, E-4, doc 215; TelCon, 19.7.76, State:HKTT; vgl. auch Miklos/Schiff//Dickman Memo, 25.5.73, FRUS 69-76, XXVII, S. 52ff.; vgl. POOLE, History of the JCS: X, S. 189-93; ähnlich retardierend behandelte man Anfragen bez. des hochmodernen, luftgestützten AWACS-Frühaufklärungssystems. Siehe Hallock Memo, 14.2.74, ,Defense [...]Set \#1 1974, Feb‘, Box 52, Schlesinger Papers, LOC; auch in der Zukunft wurden Anfragen der Iraner bez. neuer Waffenkäufe intern auf ihre Angemessenheit geprüft. Vgl. TEICHER/TEICHER, Twin Pillars, S. 30ff.

${ }^{45}$ KISSINGER, Years of Upheaval, S. 669; zudem behauptete Kissinger, sich selbst nicht mehr an die Direktive von 1972 erinnern zu können, die daher so wichtig nicht gewesen sein könne. Zudem seien $90 \%$ der Waffenverkäufe von den beiden Nachfolgeadministrationen getätigt worden, die nicht an Nixons Direktive gebunden gewesen seien. Vgl. ebd., S. 670; Verteidigungsminister Schlesinger berief 1973 einen inoffiziellen Repräsentanten, Richard Hallock, nach Teheran, um die Rüstungskäufe wenigsten etwas in geordnete Bahn zu lenken. Auch im DOD gab es Versuche seitens des Assistant Secretary of Defense for International Security Affairs, Robert Ellsworth, die Exzesse einzudämmen. Schlesinger entsandte schließlich den legendären Eric von Marbod mit ähnlichem Auftrag nach Teheran. Vgl. CIA Telegram \#sanitized to Schlesinger, 8.9.73, Schlesinger Telegram, 1.11.73, Helms Telegram, 3./4.11.73, CIA-Helms; vgl. State \#TOSEC 80313, 2.8.75, FRUS 69-76, XXVII, S. 4158; vgl. State \#183077, 2.8.75, DNSA: IR00978; SENATE SuBCOMMITTEE, Sales to Iran, S. 45.
} 
Korrekter ist eher die Interpretation, dass Nixons Zusagen vom Mai 1972 im Grunde die abschließende Bestätigung einer etablierten amerikanischen Politik seit 1964 war, in deren Rahmen kontinuierlich die wechselnden iranischen Rüstungswünsche meist nahezu vollständig akzeptiert wurden, wenn auch in der Vergangenheit meist erst im Anschluss an kleinere diplomatische Krisen und mitunter schwierige Verhandlungen. Die vorgeblich 1972 aufgegebene Steuerungs- und Kontrollfunktion war bereits mit der Aufgabe des USMilitärmonopols 1966/67 fast vollständig weggefallen - der „Blankoscheck“ konstatierte somit im Kern nur einen bereits vollzogenen Wandel im bilateralen Verhältnis. Das wichtigste Resultat war vielleicht eher die auch symbolische Aufwertung Irans zu einem gleichwertigen und formell gleichrangigen Alliierten der USA - der Versuch der Einflussnahme auf iranische Entscheidungen, zumal im sensiblen Verteidigungsbereich, und die Kontrolle derselben war mit einem solchen Verhältnis nicht mehr vereinbar und wurde nun prinzipiell aufgegeben. Hier vor allem liegt die historische Bedeutung des Nixon-Besuchs und seiner Resultate. ${ }^{46}$

\section{2. Die Nixon-Doktrin in der Praxis: Iran und die Großregion des Persischen Golfes im Kontext des britischen Abzugs}

Abseits der Rüstungsfragen versinnbildlichte Nixons Reise nach Teheran die rasant gestiegene Bedeutung Irans im globalen Netzwerk von Allianzen und special relationships der amerikanischen Führungsmacht. Noch zehn Jahre zuvor als außenpolitisch eingeschränkter Akteur wahrgenommen, dessen halbherzige Initiativen in der Region im besten Fall als Ablenkung von immer zentral erachteten Fragen interner Reform und Stabilität wahrgenommen worden waren, hatte sich Iran Anfang der 1970er Jahre zum pro-westlichen Vorzeigestaat in der Nahostregion und in der drittweltlichen Peripherie überhaupt gemausert. Bereits unter Johnson war Iran bereits als ,bright spot“" wahrgenommen worden, ein Eindruck der sich unter Nixon noch verstärkte. Kissinger verstieg sich in seinen Memoiren später zu Lobeshymnen auf den Schah: „Whatever the failings of the Shah, wrestling perhaps with forces beyond any man's control, he was for us that rarest of leaders, an unconditional ally, and one whose understanding of the world situation enhanced our own."47 Die ausufernde Preisung des strategischen Genies Mohammad Rezās in Kissingers Memoiren erfolgte zweifellos in

\footnotetext{
${ }^{46}$ Kissinger zufolge wurde Nixon ex post facto zum Sündenbock für den Bruch in den amerikanisch-iranischen Beziehungen nach der Revolution gestempelt. Tatsächlich aber sei die Entscheidung von 1972 infolge einer Einschätzung der breiteren strategischen Situation im Nahen und Mittleren Osten erfolgt. In erster Linie sei eine militärische Stärkung des unzweifelhaft pro-westlichen Irans im Angesicht einer zunehmenden sowjetischen Penetration der Region und der Aufrüstung der syrischen und irakischen Satelliten durch Moskaus beabsichtigt gewesen. Vgl. Kissinger, White House Years, S. 1263f.; siehe auch ders., Years of Upheaval, S. 524, 669.

${ }^{47}$ KISSINGER, White House Years, S. 1261 \{Hervorheb. v. Verf.\}; Kissinger gebärdete sich in seinem Memoiren empört über die mangelnde Solidarität mit Mohammad Rezā seitens späterer US-Administrationen. Vgl. ebd., S. 1258; des Weiteren preist Kissinger den Schah als aufrichtigen Reformer, der letztlich die Modernisierung seines Landes nur zu rasant vorangetrieben habe, was auch der hauptsächliche Grund für die Revolution gewesen sei. Vgl. ebd., S. 1261f.; ähnlich ders., Years of Upheaval, S. 668.
} 
apologetischer Absicht angesichts der strategisch schwerwiegenden negativen Konsequenzen der zum Zeitpunkt der Publikation ausbrechenden, iranischen Revolution.

Allerdings könnte man durchaus argumentieren, die Revolution und der Sturz der Monarchie hätten ein anderes Szenario verhindert, das sich schlüssig aus der Überrüstung seit den 1960er Jahren ergab. Nicht auszuschließen ist nämlich, dass sich, trotz immer wieder gebetsmühlenartig beschworenen „,besonderen Beziehungen“ zu Teheran und zum Schah im Besonderen, in der Zukunft die divergierenden Interessen zwischen den Verbündeten stärker ausgewirkt hätten. In der Ölfrage zeigten sich solche Tendenzen bereits- Mohammad Rezā wurde bald als einer der stärksten Preistreiber überhaupt wahrgenommen, und sein zuvor positives Image in den USA litt ab Mitte der 1970er Jahre merklich darunter. Der Wirtschaftspublizist und Autor Paul Erdman entwarf Ende 1974 das Szenario eines „Ölkriegs von 1976“. Erdmans fiktive Geschichte nahm die Megalomanie Mohammad Rezās und sein Großmachtstreben als Ausgangspunkt und entwickelte ein Szenario, im Laufe dessen die iranischen Streitkräfte, gestützt auf ein gewaltiges, vom Westen treuselig geliefertes militärisches Arsenal und auf heimlich entwickelte Atomwaffen, in einem Überraschungsschlag die gesamte Golfregion überrannten. Fortan würde der Schah dem energieabhängigen Westen seine Bedingungen diktieren. ${ }^{48}$

In der Tat gab es bei aller Begeisterung für die Bereitschaft Irans, als pro-westlicher Gendarm in der Golfregion zu agieren, auch zeitgenössische Stimmen, die vor den langfristigen Ambitionen Mohammad Rezās warnten. Bereits während der Auseinandersetzungen über Rüstungseinkäufe in den 1960er Jahren waren Bedenken zu vernehmen, welche die vorgeblich defensiven Ziele der iranischen Aufrüstung in Frage stellten. Angesichts der im Aufbau befindlichen Streitmacht, deren Umfang die militärischen Fähigkeiten aller regionalen Nachbarstaaten in den Schatten stellte, mehrten sich die mahnenden Einwürfe. Der US-Senat warnte 1976, Iran sei bereits gegenwärtig die dominante militärische Macht im Persischen Golf. Nach Eintreffen der bestellten Rüstungsgüter steige Iran spätestens 1981 zu einer ,,regional superpower" auf. ${ }^{49}$ Als wenig hilfreich wurde auch das Vorgehen der Iraner bewertet, die entgegen vorheriger Absprachen mit den Amerikanern - das neu gelieferte hochmoderne Rüstungsmaterial mit ostentativem Stolz und wohl auch mit Abschreckungsabsicht öffentlich zur Schau stellten. ${ }^{50}$ Erfahrene Beobachter wie z.B. der anerkannte Nahostkenner John

\footnotetext{
${ }^{48}$ Interessanterweise findet sich diese fiktive Geschichte auch in den Akten der CIA. Siehe Paul Erdman, ,The Oil War of 1976: How the Shah Won the World“' 2.12.74, CREST: \#RDP79*001-5; Erdman weitete die Idee 1976 in eine Dystopie in Romanform unter dem Namen The Crash of ' 79 aus. Zu Erdmans Fiktion und dem Genre allgemein vgl. YAQUB, Imperfect Strangers, S. 183-203; vgl. die gewandelte Wahrnehmung in der Presse in DORMAN/FARHANG, U.S. Press and Iran, S. 131-51.

${ }^{49}$ SenAte SubcommitTeE, Sales to Iran, S. VIII; das Oxymoron einer ,regionalen Supermacht“ findet sich bereits in internen Dokumenten aus den 1960ern. 1968 sprach Walt Rostow von einer ,, a new sub-regional super-power"; Rostow Memo, 29.4.68, FRUS 64-68, XXII, S. 508.

${ }^{50}$ Die ersten im September 1968 gelieferten $F-4$ s wurden mit einer Pressekampagne vorgestellt, die vor allem die Tatsache hervorhob, dass Iran bislang neben Großbritannien das einzige Empfängerland unter den USVerbündeten war. Vgl. Tehran \#6629, 19.9.68, DEF 12-5 IRAN, CF 67-69.
} 
Campbell sahen inhärente Widersprüche zwischen einer iranischen Dominanz der Sub-Region einerseits und der Wahrnehmung Irans als wichtiger Verbündeter andererseits. ${ }^{51}$ Der Schah selbst begann seine bisherigen Rechtfertigungen für den Aufbau einer militärischen Übermacht Irans an die sich wandelnde Lage anzupassen. Bis Ende der 1960er Jahre betonte er weiterhin die Bedrohung, die sich aus der Ausbreitung des radikalen arabischen Nationalismus in der Golfregion ergäben, insbesondere die Umtriebe Nassers, seines persönlichen bête noire. Im Dritten Arabisch-Israelischen Krieg vom Juni 1967 gab sich Iran formell neutral - so erklärte Mohammad Rezā dem wieder einmal Teheran besuchenden Averell Harriman, er könne sich nicht öffentlich gegen einen „Moslem cause“ stellen. Privat aber zeigten sich der Schah und viele iranische Offizielle geradezu entzückt über die demütigende Niederlage der Ägypter und Syrer. ${ }^{52}$ Der Schah stellte gegenüber dem Leiter der Near East and South Asia Division der CIA, James Critchfield, fest, im Kontext des Kalten Krieges lägen seine Sympathien vollständig auf Seiten Israels: ,[...] the defeat of the radical Arabs in June dramatically served Iran's interest. "53

Trotz der militärischen Schwächung der VAR hielt Mohammad Rezā anschließend an Nasser - für ihn zugleich Ausführungsorgan sowjetischer Expansion - als größter Bedrohung für Irans Sicherheit fest. Folgerichtig diente Iran als Kanal für von den Israelis erbeutete Waffen arabischer Provenienz, die nun an die royalistischen Aufständischen im Nordjemen weitergeleitet wurden, um dort Nasser und seine republikanischen Verbündeten zu bekämpfen. ${ }^{54}$ Der Abzug der Ägypter von der Arabischen Halbinsel 1967, die einseitige militärische Hegemonie Israels an der Levante, gekoppelt mit diplomatischer Rigidität und ausufernden territorialen Ambitionen sowie die zunehmende Solidarisierung der islamischen Welt mit den arabischen Frontstaaten, führten dann aber zu einer pragmatischen Anpassung der iranischen Haltung gegenüber dem Konflikt und gegenüber Ägypten. Nassers Tod 1970 und die Abschwächung pan-arabistischer Propaganda durch dessen Nachfolger Anwar as-Sādāt erleichterten das sich langsam entwickelnde Rapprochement zwischen Kairo und Teheran. ${ }^{55}$

Ausgeglichen wurde die Abschwächung der ägyptischen Bedrohung durch eine erneute

\footnotetext{
${ }^{51}$ Vgl. AMIRIE/TwITCHELL, Iran in the 1980 s, S. 329.

${ }^{52}$ Vgl. Paris \#19869, 5.6.67, FRUS 64-68, XXII, S. 385; langfristiges Ziel müsse es sein, Wege zu ergründen ,,how Nasser could be destroyed"; ebd.; vgl. CIA Intel Memo, 18.8.67, ebd., S. 411; vollständige Version in CREST: \#RDP79*001-4; allerdings genehmigte der Schah einen Überflug sowjetischer MiGs durch iranischen Luftraum als Nachschub für die irakische Luftwaffe, um den Anschein von Ausgeglichenheit zu wahren. Vgl. Tehran \#5265, 30.6.67, , Iran Cables, Vol. II, [1 of 2]; 1/66-1/69', NSF, Country File, Box 136, LBJL.

${ }_{53}$ Critchfield Memo, 16.11.67, FRUS 64-68, XXII, S. 439ff.; weitere Freigaben zuvor klassifizierter Teile dieses Dokuments infolge eines FOIA-Antrags (F-2013-00185) des Verfassers.

${ }^{54}$ Vgl. ebd.; Humphrey Memo, 24.8.67; FRUS 64-68, XXII, S. 425f.

${ }^{55}$ Vgl. RaMAZANI, Iran and the Arab-Israeli Conflict; im Anschluss an den Krieg von 1973 und angesichts der verbesserten Beziehungen zu Ägypten nach dem Tod Nassers gab sich der Schah weitaus kühler gegenüber dem israelischen Partner und verteidigte auch öffentlich die arabische Position. Vgl. CIA Memo ,Iran's Mediatory Role in Middle East", 29.5.75, CREST: \#RDP86*019-4; die Botschaft berichtete von einem ,real growth of sympathy for Arab cause among all segments of Iranian population“, vielleicht auch ein weiterer Hinweis auf den zunehmenden Einfluss religiöser Überzeugungen auf die politische Ausrichtung. Siehe FRUS 69-76, XXVII, S. 148 n3; vgl. CIA Telegram \#sanitized to Scowcroft, 30.10.73, CIA-Helms; vgl. PARSI, Iran's Arab Option.
} 
Eskalation im Verhältnis zu Irak. Im Anschluss an den zweiten Staatsstreich der Baath-Partei 1968 verschlechterten sich die beiderseitigen Beziehungen rapide. Wieder einmal waren es die Kontroversen um die ungelöste Šațț al-'Arab-Frage, die beide Länder im Frühling 1969 an den Rand eines militärischen Konflikts brachten. ${ }^{56}$ Die Amerikaner registrierten, dass die Verschärfung des Konflikts bewusst durch Iran inszeniert worden war, offenbar ermuntert durch das immer größer werdende militärische Ungleichgewicht zwischen den beiden Staaten. Der Beinahe-Zusammenstoß mit Irak demonstrierte für Washington die fehlgeleiteten und widersprüchlichen Annahmen hinter der als stabilisierend intendierten Aufrüstung des iranischen Verbündeten. Mohammad Rezā verweigerte sich den Einwänden der USA, der Irak stelle angesichts der iranischen militärischen Überlegenheit im Grunde keine echte Bedrohung dar. Stattdessen zeichnete er Irak nunmehr als willfährigen sowjetischen Satelliten, der dabei sei, Eckstein der regionalen Strategie Moskaus zu werden, selbstverständlich langfristig gerichtet auf die Kontrolle des Golfs und die Verweigerung seiner Ölreserven für die westlichen Industriestaaten. ${ }^{57}$ Die iranische Pressionsstrategie führte in einer Art sich selbst erfüllender Prophezeiung zu einer Annäherung Bagdads an die UdSSR, wenige Jahre später kulminierend im Freundschaftsvertrag vom April 1972. ${ }^{58}$ Angesichts der antikommunistischen Überzeugungen der oberen Baath-Führung ist zu bezweifeln, ob Irak auch ohne iranischen Druck diesen Kurswechsel, zumindest in dieser Schärfe, vollzogen hätte. Auf die Konfrontation von 1969 folgte ein rasant einsetzender Rüstungswettlauf - wie einige Jahre zuvor von Senator Fulbright vorhergesagt -, ein eskalierender Grenzkrieg mit Scharmützeln und Artillerieduellen und gegenseitige Subversionskampagnen in Xuzestān und Kurdistan. Die iranische Überlegenheit zwang Irak letztlich zur Aufgabe: Im März 1975 unterzeichneten der irakische Machthaber Saddam Hussein und Mohammad Rezā eine Vereinbarung in Algiers, welche die Šațt al-'Arab-Frage gemäß den iranischen Vorstellungen regelte. ${ }^{59}$ Was als gütliche Regelung eines Jahrzehnte alten Konflikts gefeiert wurde, war indes nur Katalysator einer noch intensiver werdenden Rivalität zwischen beiden Staaten, die sich nach der Revolution im verlustreichen achtjährigen Iran-Irak-Krieg entladen sollte. ${ }^{60}$

Die sich bereits Ende der 1960er Jahre abzeichnende militärische Dominanz der Sub-Region warf in der Tat Fragen bezüglich der Nachhaltigkeit der seitens Washington verfolgten Strategie auf, die im Kern auf eine Unterstützung eines iranischen Primats im Golf nach dem britischen

\footnotetext{
${ }^{56}$ INR betonte: „The latest crisis over the Shatt al-Arab has been characterized so far by relative Iraqi moderation and Iranian belligerence.“; vgl. INR Intel Note No. 295, 22.4.69, FRUS 69-76, E-4, doc. 11; vgl. Tehran \#1371, 18.4.69, ebd., doc. 12; vgl. AlvANDI, Origins of Primacy, S. 350-3; vgl. Eintrag vom 17.4.69, in: AlAM, Shah and I, S. 52f.; Teheran erklärte im April den Grenzvertrag von 1937 für ungültig.

${ }^{57}$ Vgl. Tehran \#1925/\#4183, 19.5./13.10.69, FRUS 69-76, E-4, doc. 17, 23.

58 Vgl. Eliot Jr. Memo, 13.4.72, ebd., doc. 305; gegenüber Premier Heath konstruierte der Schah den Freundschaftsvertrag umgehend als Beleg für eine neue Bedrohungskonstellation, da dieser angeblich die Stationierung von Truppen im Irak erlaube. Record of Conversation [Heath, Schah], 23.6.72, PREM 15/991, TNA:PRO.

${ }^{59}$ Vgl. CIA Study ,The Implications of the Iran-Iraq Agreement', 1.5.75, CREST: \#RDP79*002-7; vgl. DIA Notice DIADIN 529-75, 7.3.75, FRUS 69-76, XXVII, S. 744f.

${ }^{60}$ Zur fortgesetzten Rivalität vgl. CIA Study ,Persian Gulf*, 5.4.77, CREST: \#RDP81*002-9.
} 
Abzug hinauslief. Der Schah wiederum vermied in Gesprächen mit amerikanischen Offiziellen jedes Eingeständnis, dass er ein eben solches anstrebe. In durchsichtiger Anbiederung an die amerikanische globale Eindämmungsstrategie stilisierte er sich als pro-westliches Bollwerk gegen eine doppelte sowjetische Zangenbewegung gegen den Golf, die rechte Zange mittels der VAR und des kommunistischen Südjemens auf die Kontrolle der Arabischen Halbinsel gerichtet, die linke durch den irakischen Satelliten direkt auf den Golf selbst abzielend. Auf die Bemerkung des amerikanischen Secretary of State William Rogers im Oktober 1969 hin, Iran sei bereits jetzt „much stronger than Iraq and would it not be madness for Iraq to contemplate attacking Iran“, beharrte der Schah auf der Notwendigkeit einer ,over-kill capability“ mit dem Ziel der Abschreckung: ,[...] those fellows in Iraq are mad. “61 Mohammad Rezā versuchte, jeden Argwohn auf amerikanischer Seite über seine letztendlichen strategischen Ziele zu zerstreuen. Anfang 1967 versicherte er General Conway, Iran strebe keinerlei „territorial aggrandizement“ an. Im Oktober 1969 bestritt er gegenüber CIA-Direktor Richard Helms ein weiteres Mal, dass Iran auch nur einen Zentimeter fremden Bodens begehre. Zweck der Aufrüstung sei einzig und allein die Abschreckung einer möglichen Abenteuerpolitik durch die Nachbarn. Ein Jahr später verteidigte er seine Rüstungsanstrengungen erneut als defensiv ausgerichtet. Er habe keineswegs im Sinn, eine große imperiale Streitmacht ,wie Kaiser Wilhelm oder Hitler“ zu erschaffen. ${ }^{62}$

Diesen gebetsmühlenartig wiederholten Beteuerungen schenkten nicht alle Akteure Glauben. Aus Sicht der Golfaraber, so ein kundiger NEA-Offizieller seien umfangreiche iranische Streitkräfte ,likely to prove not only unnecessary but [...] positively harmful. “63 Der saudische König Fayṣal zweifelte an der Notwendigkeit von Rüstungsanstrengungen in diesem Ausmaß und spekulierte über deren letztendliche Verwendung für aggressive Zwecke. ${ }^{64}$ Tatsächlich warf der sich abzeichnende Umfang der iranischen Streitmacht einige Fragen auf. Nach dem Niedrigstwert von 162.000 Mann im März 1965 - umgesetzt aufgrund amerikanischen Drucks - wurden die iranischen Streitkräfte bis Ende 1970 langsam bis auf 183.000 Mann aufgestockt. Danach aber wurde der Anstieg rasant. Bis Mitte der 1970er Jahre waren die Streitkräfte auf insgesamt 380.000 Mann angewachsen, also fast das Doppelte der 1962 vereinbarten Sollstärken-Obergrenze. ${ }^{65} 1974$ verfügte Iran bereits über 180 F-4- und F-5-Kampffugzeuge

\footnotetext{
${ }_{61}$ MemCon, 22.10.69, FRUS 69-76, E-4, doc. 33; die CIA widersprach der Darstellung Iraks als Satelliten Moskaus. Zudem schätzten die Sowjets die positiven Konsequenzen ihres Rapprochements mit Iran und hätten daher ein Interesse, nicht allzu enge Beziehungen zu Teherans Erzfeind einzugehen. Vgl. Saunders/Hoskinson Memo, 17.5.72, ebd., doc. 194; INR im State Department bezweifelte die Begründung der iranischen Aufrüstung basierend auf angeblicher externer Bedrohung. Vgl. INR Research Study RNAS-6, 9.6.70, DNSA: IR00734.

${ }^{62}$ Tehran \#3258, 15.2.67, FRUS 64-68, XXII, S. 352; vgl. Helms Memo for the Record, 22.10.69; Tehran \#5335, 10.12.70, FRUS 69-76, E-4, docs. 31, 106; vgl. INR Research Memo RNA-35, 7.11.68, POL 1 IRAN, CF 67-69. ${ }^{63}$ Brewer Memo, 27.2.70, FRUS 69-76, E-4, doc. 51.

${ }^{64}$ Vgl. CIA Memo No. 1290/74, 4.6.74, CREST: \#RDP85*004-6; ARMISH/MAAG bestätigte den denkbaren Einsatz der Special Forces und der modernisierten Luftlandeeinheiten zur Machtprojektion in der Golfregion. Vgl. Tehran \#1052, 23.3.70, CREST: \#RDP72*043-8.

${ }^{65}$ Es gab bis 1970 eine versteckte Sollstärkenerhöhung über den Umweg der Gendarmerie, die nun 67.000 Mann umfasste. Vgl. CIA, SNIE 34-70, 3.9.70, FRUS 69-76, E-4, doc. 86; vgl. CIA Elites Study, S. 47; vgl. BILL, Eagle and Lion, S. 202.
} 
und hatte 280 Neubestellungen für beide Flugzeugtypen in Auftrag gegeben. Hinzu kam im Anschluss an den „Blankoscheck“ eine Order über 30 - bald gesteigert auf 80 - des sich noch in der Entwicklung befindlichen, modernsten Kampfflugzeugs seiner Zeit, der F-14 Tomcat. Die Luftverteidigung Irans wurde gleichzeitig mit zwei Batterien britischer Rapier-Boden-LuftRaketen, über 1.000 amerikanischen $H A W K$-Luftabwehrraketen und modernsten Radarsystemen verbessert. Die Marine war mit Abstand die stärkste aller Anrainerstaaten im Persischen Golf. Neben einem alten, ehemals britischen Zerstörer unterhielt die kaiserliche Marine zwei kleinere amerikanische Lenkwaffenzerstörer und vier schnelle Fregatten. Zwei weitere moderne US-Zerstörer waren bereits bestellt. Hinzu kam die weltweit größte Flottille von Luftkissenbooten. ${ }^{66}$

Secretary of Defense Melvin Laird hatte bereits im Juli und dann ein weiteres Mal im Oktober 1970 festgestellt, dass die bestehenden iranischen Streitkräfte in jeder Hinsicht ausreichend für sämtliche vorstellbaren Verteidigungsszenarien seien. Er warnte eindrücklich vor den strategischen Folgen einer Fortsetzung dieser Politik:

To agree to his full-purchase program would, to my mind, be destabilizing in the Persian Gulf area, lead his neighbors - principally Iraq - to increase their arms inventories, strain Iran's financial and personnel resources, and present new opportunities for the Soviets and radical Arab states to penetrate the area. ${ }^{67}$

Wie bereits beschrieben, stießen diese Bedenken in den internen Beratungen der NixonAdministration auf wenig Entgegenkommen. Der Schah bestellte nun 36 zusätzliche Lockheed C-130 Hercules-Transportflugzeuge, offenkundig mit dem Hintergedanken in Saudi-Arabien intervenieren zu können, so die Einschätzung von JCS-Chairman General Wheeler. ${ }^{68}$ Angesichts der Befürchtungen über die langfristigen Bestrebungen Teherans in der Golfregion war die fast schon arglos zu nennende Fortsetzung der Aufrüstungspolitik gegenüber Iran doch überraschend. Allerdings dürfen bei der Bewertung dieser Politik die bestehenden Zwänge und Umstände, mit denen sich die USA konfrontiert sahen, nicht übersehen werden.

Im Januar 1968 hatte die britische Labour-Regierung unter Harold Wilson den Rückzug East of Suez - aus dem Persischen Golf, aus Singapur und aus Malaysia - bis Ende des Jahres 1971

\footnotetext{
${ }^{66}$ Vgl CIA Intel Memo ,Iran: The Shah's Economic and Military Expansion‘, Februar 1973; CIA Weekly Review Special Report ,Iran: Building Up the Military“, 7.6.74, CREST: \#RDP85*002-3; Andrew Memo, 24.7.72, PREM 15/990, TNA:PRO.

${ }^{67}$ Laird Letter, 7.10.70, FRUS 69-76, E-4, doc. 93; im Juli sprach Laird von der Schwierigkeit „,to balance an almost total lack of military requirements for additional aircraft against the likelihood that our close relationship with Iran would be damaged if we were to deny the Shah's request."; Laird Letter, 27.7.70, DEF 12-5 IRAN, CF $70-73$.

${ }^{68}$ MemCon, 14.4.70, FRUS 69-76, E-4, doc. 61; tatsächlich bestätigte Mohammad Rezā selbst diese angedachte Verwendung während seines Staatsbesuchs vom Sommer 1973. Auf die geäußerte Sorge Kissingers hin, wie auf einen Staatsstreich eines neuen ,Qad̄ d̄āfi“" in Kuwait oder Saudi-Arabien zu reagieren wäre, antwortete der Schah: „If there were an illegal coup, I could have my parachute brigade and some of my C-130s in Saudi Arabia in a very short period of time.", MemCon, FRUS 69-76, XXVII, S. 100.
} 
angekündigt. Begründet mit den finanziellen Belastungen, die sich aus einer Fortsetzung der britischen Weltmachtrolle im tradierten Umfang ergäben, lagen die wahren Gründe für die britische Entscheidung wohl woanders. ${ }^{69}$ Während einige Autoren an vorwiegend innenpolitische Beweggründe glauben, waren wohl eher breitere strategische Überlegungen für die Entscheidung maßgeblich. Dafür spricht auch, dass die konservative Partei nach ihrer Regierungsübernahme - wenn auch nach einigem Zögern - am Abzug festhielt, trotz ihrer scharfen Kritik an der vorherigen Entscheidung der Labour-Regierung. ${ }^{70}$ Für die amerikanische Politik stellte sich nun die Frage der zukünftigen Ordnung in der Großregion des Persischen Golfes. Großbritannien hatte bereits 1967 den südlichen Jemen geräumt, wo sich rasch marxistische Kräfte durchgesetzt und den einzigen kommunistischen Staat im Nahen und Mittleren Osten etabliert hatten. ${ }^{71}$ Eine ähnliche Entwicklung im Golf selbst wäre ohne Frage aus amerikanischer Sicht nicht hinnehmbar gewesen und hätte die seit dem Zweiten Weltkrieg gegenüber der Sowjetunion verfolgte Eindämmungspolitik in der Region unterminiert. Allerdings waren die politischen Gegebenheiten im Golf gänzlich anders gelagert als in Südarabien. Vereinzelt gab es radikale Kräfte und insbesondere Irak stand im Verdacht der heimlichen Unterstützung derartiger Subversion. ${ }^{72}$ Insgesamt aber dominierten in den bisher unter britischer Schutzherrschaft stehenden Golfemiraten der Vertragsküste sowie in Katar und Bahrain - weitgehend unangefochten - konservative Kräfte. Naheliegend war daher die Überführung der bisherigen Protektoratsbeziehungen in die Unabhängigkeit nach dem erfolgreichen Beispiel Kuwaits, bevorzugt in einer gemeinsamen Union aller neun

\footnotetext{
${ }^{69}$ Vgl. Cabinet Conclusions, 4.1.68, BDEE A, 5, I, S. 120-7; vgl. JIC (B)(71)9, 6.8.71, CAB 188/13, TNA:PRO; BALFOUR-PAUL, End of Empire, S. 124, zufolge beliefen sich die jährlichen Militärausgaben Großbritanniens im Golf auf nur etwa $£ 12$ Millionen, somit eine Summe, die durchaus zu schultern gewesen wäre. Das Angebot der „Rulers“ der Vertragsküste, für die Kosten in der Zukunft aufzukommen, wurde ebenfalls abgelehnt. Vgl. MemCon, 11.1.68, ,United Kingdom, Vol. XIII, [...]2]', NSF, Country File, Box 212; Saunders Memo, 14.6.68, ,Saunders Memos', NSF, Name File, Box 7, LBJL.

${ }_{70}$ Allerdings hatten sich die Anrainerstaaten nun bereits auf einen Abzug eingestellt. Nach dem Amtsantritt der Tories erklärten Iran, Saudi-Arabien und Kuwait rasch ihre Ablehnung eines Verbleibs der britischen Macht im Golf. Tatsächlich war man nun auch im US-State Department gegen einen Kurswechsel in der Frage der britischen Präsenz. Vgl. Neubert Memo, 24.6.70, POL 33 PERSIAN GULF, CF 70-73; JIC(A)(70)49, 8.12.70, CAB 186/6, TNA:PRO; vgl. BALFOUR-PAUL, End of Empire, S. 128f,; inzwischen gibt es eine Fülle von Interpretationen für die Ursachen des ,withdrawal East of Suez“. Die Rolle der Innenpolitik, insbesondere des linken Labour-Flügels betonend GAUSE, British and American Policies, S. 253; ähnlich SATO, Decision to Withdraw; den innenpolitischen Druck als finalen Auslöser einer sich inkrementell entwickelnden Entscheidung bei FAIN, Ascendance and Retreat, S. 161-8; und PICKERING, Politics of Retrenchment; PETERSEN, Decline, S. 2, 111-25; vertritt die zweifelhafte These einer britischen Entschlossenheit zum Abzug vom Amtsantritt Wilsons an. LOUIS, British Withdrawal, sieht ein Zusammenspiel von wirtschaftlichem Niedergang und imperialen Ermüdungserscheinungen; DOCKRILl, Britain's Retreat, sieht eine grundsätzliche Kehrtwende der britischen Grand Strategy weg vom bisherigen Globalismus; MCCOURT, East of Suez Role, sieht ein langsames Eingeständnis der strategischen Sinnlosigkeit der eigenen Truppenpräsenz in der Peripherie. Ähnlich SMITH, Pattern Not a Puzzle.

${ }^{71}$ London hatte auf den Abzug aus Aden mit einer Aufstockung der eigenen Präsenz im Golf selbst reagiert, um Befürchtungen über einen anstehenden Rückzug aus der gesamten Region zu zerstreuen und die verbündeten Herrscher im Golf selbst rückzuversichern. Vg. London \#A-1927, 12.12.67, ,United Kingdom, Cables, Vol. XII, 7-12/67', NSF, Country File, Box 211; F. , The Persian Gulf‘, Administrative History of the Department of State, Vol. I, Ch. 4, 5, 6, Box 2 [1 of 2], Chapter 4 [...]G', Special Files, Administrative Histories, LBJL \{im Folgenden: Administrative History/Persian Gulf $\}$, S. 2, 4f.; Roberts Report to Brown, 17.11.67, BDEE A, 5, I, S. 118f.

${ }^{72} \mathrm{Vgl}$. CIA Intel Report ,Security and Subversion in the Persian Gulf', 1.3.68, ,Middle East, Vol. I [1 of 2] ${ }^{\circ}$, NSF, Country File, Middle East, Box 104, LBJL; im Herbst 1970 landete, wahrscheinlich mit irakischer Unterstützung, eine Gruppe von 70 bewaffneten Dissidenten in der omanischen Exklave Musandam in der Meerenge von Hormus mit dem Ziel den Emir von Sharjah (Al-Šāriqa) und anschließend den Sultan von Oman zu stürzen. Mit britischer militärischer Unterstützung wurde die kleine Rebellion niedergeschlagen. Vgl. DOP(70) 11th Meeting, Item 4,
} 30.11.70, CAB 148/101, TNA:PRO. 


\section{Golfemirate. $^{73}$}

Um eine solche friedfertige Transition in eine neue regionale Ordnung zu ermöglichen, bedurfte es allerdings der Zustimmung von wenigstens zweien der drei großen Anrainerstaaten, von Iran und Saudi-Arabien. Irak war aufgrund seiner internen revolutionären Umgestaltungen seit dem Sturz der haschemitischen Monarchie 1958 und seiner außenpolitischen Nähe zum Ostblock ohnehin isoliert. Allerdings hatte es London in den vorangegangenen Jahrzehnten versäumt, ernsthaft an einer Lösung der bestehenden Grenzkonflikte zu arbeiten, zumal der ungeklärte Buraimi-Konflikt zwischen Saudi-Arabien und Abu Dhabi bereits einen acht Jahre andauernden Abbruch der diplomatischen Beziehungen zu Riad bis 1963 zur Folge gehabt hatte. Saudi-Arabien beanspruchte weiterhin einen großen Teil des Territoriums der geplanten Föderation arabischer Emirate. Ein weiteres ungelöstes Problem war der iranische Anspruch auf Bahrain und auf einige strategisch bedeutsame Inseln im Golf selbst. Gemeinsam mit dem Šațt al-'Arab-Konflikt und dem irakischen Anspruch auf Kuwait und der noch weitgehend ungelösten Streitfragen über den Verlauf der Seegrenzen und der Abgrenzung der Festlandssockel besaßen alle diese Territorialkonflikte Potential für eine Destabilisierung der Sub-Region. ${ }^{74}$

Die amerikanische Politik hatte bereits seit den frühen 1960er Jahren auf den Verbleib der britischen Ordnungsmacht im Golf hingearbeitet. Angesichts der fortgesetzten Schwäche des Pfund Sterling und der drohenden Abwertung der Währung mit resultierenden Einschnitten in den Verteidigungshaushalt hatten die USA bereits mehrfach finanzielle Unterstützung geleistet. ${ }^{75}$ Noch kurz vor der Verlautbarung des britischen Rückzugs hatte Präsident Johnson auf Wilson eingewirkt, die Entscheidung nochmals zu überdenken: „If these steps are taken, they will be tantamount to British withdrawal from world affairs, with all that means for the future safety and health of the free world."76 Konfrontiert mit der Entschlossenheit Londons, die verbliebenen nahöstlichen (und südostasiatischen) Positionen aufzugeben, besann sich die Johnson-Administration letztlich zu einer Kehrtwende und sprach sich nunmehr für die

\footnotetext{
${ }^{73}$ Formal waren die Golfstaaten aufgrund der Exclusive Agreements von 1892 mit den Scheichs der Piratenküste und vergleichbaren Vereinbarungen mit den anderen Emiraten Protected States, aber keine Protectorates. Vgl. BALFOUR-PAUL, End of Empire, S. 101f.

${ }^{74}$ Vgl. auch Tehran \#A-523/\#A-579, 29.3.67/7.5.68, POL 33 PERSIAN GULF, CF 67-69.

${ }^{75}$ In der allgemeinen Literatur dominiert weiterhin die Fehlinterpretation einer (aktiven) Ablösung der britischen Vormachtrolle durch die USA im Nachgang zur Suezkrise - tatsächlich waren die USA vielmehr an einem Weiterbestehen des Pax Britannica im Persischen Golf interessiert. Ein wichtiges Korrektiv der herkömmlichen Sichtweise ist SMITH, Power Transferred.

${ }_{76}$ Johnson Letter, 11.1.68, FRUS 64-68, XII, S. 609; vgl. auch Battle Briefing Memo, 9.1.68, FRUS 64-68, XXI, S. 256ff.; Walt Rostow hatte noch einen zusätzlichen scharfen Passus für den Brief vorgeschlagen, mit Bezug auf ,certain actions we might be forced to take with respect to arrangements which have rested on the assumption that Britain was carrying a part of or common global responsibilities", wohl ein Hinweis auf eine mögliche Aufkündigung der engen nachrichtendienstlichen und nuklearen Kooperation. Rostow Memo, 11.1.68, ,Uni[...] XIII, Memos, 1/68-7/68 [2 of 2]', NSF, Country File, Box 212, LBJL.; Dean Rusk forderte den britischen Außenminister George Brown am gleichen Tag auf „For God's Sake, Act Like Britain!“; in den US-Quellen findet sich nur die Bemerkung ,,be Britain“. Rusk ging noch weiter und bemerkte ,he was disturbed when the teacher abandoned the field."; siehe MemCon, 11.1.68, ebd., die längere Version zit. aus FAIN, Ascendance and Retreat, S. 141 .
} 
Etablierung einer stabilen regionalen Ordnung, basierend auf der Kooperation der Anrainerstaaten, aus. „Don’t Mourn, Organize“, erklärte Sicherheitsberater Rostow nur wenige Tage später und machte erste Vorschläge für eine neue Ordnung. Iran und Saudi-Arabien sollten gemeinsam das entstehende Machtvakuum füllen: „We shall have to move carefully, but we might give them both encouragement and sell them arms. “"77

Hier lagen die Anfänge der strategischen Neuausrichtung, die später - aber nie zeitgenössisch ${ }^{78}$ - als die sogenannte Twin Pillars-Politik bezeichnet werden sollte. Die JohnsonAdministration stimmte in den folgenden Monaten ihre Golfstrategie eng mit der bisherigen Hegemonialmacht Großbritannien ab mit dem Ziel, eine stabile Ordnung für die Zeit nach dem britischen Abzug zu errichten. Rasch zeigte sich, dass Mohammad Rezās Ambitionen eines der Haupthindernisse für die zukünftige Stabilität sein würden, denn der Schah sah nun endlich den Zeitpunkt für die Umsetzung seiner grandiosen Pläne für die Stellung Irans in der Golfregion gekommen. Der Schah begrüße den britischen Abzug ,[...] as a new era with glorious possibilities for Iran“, so die US-Botschaft. ${ }^{79}$ Gleichzeitig war sich Mohammad Rezā bewusst, dass eine allzu abenteuerliche Golfpolitik die amerikanisch-iranischen Beziehungen belasten könnte. In der Tat führte seine „Kanonenbootpolitik“ gegenüber Saudi-Arabien in den ersten Monaten des Jahres 1968 zu einigen Verstimmungen im Verhältnis zu Washington. Schriftlich aufgefordert von Präsident Johnson, „Geduld und staatsmännische Umsicht“ gegenüber den anderen Golfstaaten zu demonstrierten, reagierte Mohammad Rezā in seiner Antwort gereizt und versprach fortgesetzte Geduld, ,until the parties concerned come to their senses.“ Zugleich kündigt er hier zum ersten Mal die neue Leitlinie zukünftiger iranischer Golfpolitik an. Iran lehne die Involvierung extra-regionaler Mächte bei der Wahrung von Ordnung und Frieden in der Golfregion ab - dies schloss nicht nur eine weitere Präsenz Großbritanniens und die Ablehnung jeglicher sowjetischer Involvierung aus. Die neue Richtlinie war zugleich gegen eine unmittelbare Stabilisierungsrolle der USA im Golf gerichtet. $^{80}$

Bereits im Februar 1968 hatte man sich in der IRG/NEA auf die Förderung intra-regionaler Kooperation zur Sicherung der Stabilität geeinigt, zuvorderst die Lösung der verschiedenen Streitigkeiten zwischen Saudi-Arabien und Iran anstrebend. Zugleich hoffte man auf eine Beibehaltung der britischen Präsenz jenseits militärischer Machtprojektion, insbesondere die

\footnotetext{
${ }^{77}$ Rostow Memo, 16.1.68, ,Rostow Memos [1 of 2]', NSF, Name File, Box 7, LBJL; vgl. Dean Despatch to Brown, 4.3.68, BDEE A, 5, I, S. 146-50.

${ }^{78}$ Vgl. Alvand, Origins of Primacy, S. $338 n 3$.

${ }^{79}$ Tehran \#3626, 6.3.68, FRUS 64-68, XXII, S. 472.

${ }^{80}$ Shah Letter, 1.2.68; Johnson Letter, 1.2.68, FRUS 64-68, XXI, S. 271, 269f.; vgl. Rostow Memo, 31.1.68, ebd., S. 268f.; „Kanonenbootpolitik“ in Telegram \#3626, vorherig. Anm.; gemeint war die provokative Haltung im Festlandssockelstreit mit Saudi-Arabien und die Absage eines Staatsbesuchs durch den Schah aus Verärgerung über die saudische Haltung. Beunruhigend waren zudem Berichte über iranische Überlegungen, den Persischen Golf zu einem Binnengewässer zu erklären, und damit die auch nur vorübergehende Flottenpräsenz extraregionaler Mächte zu delegitimieren. Vgl. State \#CA-3729, 10.7.70, POL 33 PERSIAN GULF, CF 70-73.
} 
Fortsetzung der Beraterrolle Londons bei den jungen Sicherheitskräften der bald unabhängigen Emirate. Für die USA selbst sah man abgesehen von der fortzusetzenden „leading role“ in Iran und Saudi-Arabien nur eine vergleichsweise „low-key“-Involvierung in eigentliche Golfangelegenheiten. ${ }^{81}$ Zugleich wurde eine begrenzte Verbesserung der eigenen Fähigkeiten zur militärischen Machtprojektion angedacht, als Rückversicherung für die pro-westlichen Golfherrscher gegen mögliche ägyptische oder sowjetische Ambitionen. Neben der Beibehaltung der symbolischen Flottenpräsenz seit den späten 1940er Jahren durch MIDEASTFOR erwog man die Bereitstellung begrenzter Luft- und Bodenkapazitäten zusätzlich zu navaler Präsenz im Indischen Ozean, später umgesetzt in der sogenannten Overthe-Horizon-Präsenz in Diego Garcia, dessen Ausbau zu einem größeren Stützpunkt fast zeitgleich beschlossen wurde. Die Umsetzung dieser Pläne bedeuteten nicht die Übernahme der britischen Rolle im Golf, schaffte aber dafür den Nukleus für die Wahrnehmung einer solchen Option in der Zukunft. Der implizite Widerspruch zur offiziellen Haltung, der zufolge man nicht beabsichtige, das entstehende „Vakuum“ selbst zu füllen, scheint aber selbst den Zeitgenossen nicht wirklich bewusst gewesen zu sein. ${ }^{82}$ Zusätzlichen Antrieb bekamen diese Pläne durch umtriebige diplomatische Aktivitäten der UdSSR nach Ankündigung des britischen Abzugs. Der Chef des sowjetischen Generalstabs Sacharow besuchte Teheran im Mai und offerierte sowjetische Waffen - Zerstörer, U-Boote und MiG-21 - in großem Umfang zu attraktiven Konditionen. Im Mai und Juni 1968 besuchten erstmalig seit 1903 sowjetische (russische) Flottenverbände iranische und irakische Häfen. INR im State Department aber zeigte sich angesichts des neuartigen sowjetischen Engagements gelassen - auch Moskau sei gezwungen, seine Interessen auszubalancieren. Eine direkte Unterstützung iranischer Ambitionen sei mit dem eigenen engen Verhältnis zu Bagdad letztlich nicht vereinbar. ${ }^{83}$

\footnotetext{
${ }^{81} \mathrm{Vgl}$. Record of IRG/NEA Meeting, 1.2.68, FRUS 64-68, XXI, S. 272f.; ausdrücklich lehnte man ab, die Briten zu ,ersetzen“. Die neue Politik wurde folgendermaßen zusammengefasst: „Our policy should be directed along the lines of (a) encouraging the British to maintain as much of their present special role in the Gulf as they can, as long as possible (including their role as principal arms supplier to various Gulf states); (b) encouraging the Saudis and Iranians to settle their outstanding differences regarding the median line and other issues; (c) encouraging greater political and economic cooperation generally among the Gulf states; and (d) avoiding an undue military buildup by Gulf littoral states.“; ebd., S. 273; Punkt (b) fehlt in späteren Dokumenten, offenbar der Abneigung gegen eine eigene Involvierung in die Streitfragen geschuldet. Die CIA war pessimistisch bez. vertrauensvoller Kooperation zwischen Saudi-Arabien und Iran. Vgl. CIA Special Memo , 1.2.68, ,Middle East, Vol. I [1 of 2], 6/65-3/68', NSF, Country File, Middle East, Box 104, LBJL; zur Beibehaltung einer reduzierten britischen Präsenz vgl. Crocker Memo, 12.10.70, DDRS: CK3100653165.

${ }^{82}$ Vgl. State Study, ,The Politico-Military Problem for the US in the Arabian Sea Littoral', 15.4.68, FRUS 64-68, XXI, S. 289ff.; hier auch die erstmalige Erwähnung von „over the horizon“. Zur Wahrnehmung der sowjetischen Ambitionen vgl. Warnke Memo, ebd., S. 296f;; IRG/NEA Meeting Record, 10.7.68, ebd., S. 299ff; zu Diego Garcia vgl. Office of the Chief of Naval Operations Paper, 11.2.70, FRUS 69-76, XXIV, S. 126-9; TelCon, 10.6.70, DNSA:HAKTelCons: \#KA03094; Kullis Memo, 5.3.71, fol. 26, CAB 164/904, TNA:PRO; FAIN, Indian Ocean Region, S. 7f., 11-6; zur US-Präsenz auf Bahrain vgl. GRESH, Gulf Security and the U.S. Military, S. 91-116; WINKLER, Bahrain, S. 45-62.

${ }^{83}$ Die USA verweigerten sich anfangs konsequent den Annäherungsversuchen der neun Emirate und auch Omans. So lehnte man eine kuwaitische Anfrage zum Kauf amerikanischer Waffen ab. Vgl. Schwartz Memo, 22.4.68, FRUS 64-68, XXI, S. 292f.; vgl. dagegen JCS Memo, 19.6.68; Nitze Memo, 2.8.68, ebd., S. 298f., 303f.; zur sowjetischen Rolle vgl. INR Intel Note No. 434, 6.6.68, ,Iran, Visit [...]12/68‘, NSF, Country File, Box 137; Black Memo, 14.2.68, ,Middle East, Vol. 1 [1 of 2]; 6/65-3/68', NSF, Country File, Box 104, LBJL; State \#CA-6701, 17.12.69, FRUS 69-76, XXIV, S. 49-55; CIA Intel Memo No. 0865/72, , Moscow and the Persian Gulf‘, 12.5.72, DDRS: CK3100228362; JIC(A)(71)7 ,Future Soviet Policy in the Persian Gulf*, 16.3.71, CAB 186/8, TNA:PRO; Wilson Minute, 29.3.71, DBPO III, I, S. 322-6.
} 
Jenseits dieser - ohnehin begrenzten - Anpassungen der eigenen Position hielt man sich an die vereinbarte Politik der weitgehenden Nicht-Involvierung. So blieb es Großbritannien vorbehalten, eine Lösung der offenen Fragen zu versuchen. In der Tat gelang es im September 1968 die Frage der Abgrenzung der Festlandssockel im Persischen Golf zwischen SaudiArabien und Iran zu schlichten. ${ }^{84}$ Die formelle Gründung einer Federation of Arab Amirates im März 1968 - wenngleich nie wirklich umgesetzt - durch alle arabischen Herrscher der verbliebenen ,protected states“, also einschließlich Katars und des iranisch beanspruchten Bahrains, führte allerdings $\mathrm{zu}$ neuen Problemen und einer schweren Belastung des Verhältnisses zwischen Iran und Großbritannien. Die USA nahmen auch hier demonstrativ eine neutrale Haltung ein und rieten zu Geduld und Verhandlungsbereitschaft. ${ }^{85}$ Neben Bahrain kehrte nun auch die „Inselfrage“ als Streitpunkt zurück. Wie in Kapitel VII.1. beschrieben, gab es zwischen Golfarabern und Iran eine Reihe von umstrittenen Inseln, die wichtigsten davon das vom Emirat Sharjah (Al-Šāriqa) beanspruchte Abu Musā sowie die Große und die Kleine Tunb-Insel, von Ra's al-ㅂajmah als eigener Besitz angesehen. Iran betrachtete alle drei Inseln als widerrechtlich von Großbritannien vom iranischen Mutterland abgetrennt und strebte eine Rückgewinnung an. Die Inseln lagen strategisch günstig am westlichen Ausgang der Straße von Hormus und waren somit von großer Bedeutung für das Ziel des Schahs, die unangefochtene militärische Vormacht im Golf zu werden. ${ }^{86}$ Britische Beobachter registrierten ebenfalls die Absicht Mohammad Rezās, seinen Einfluss auf die Vertragsküste auszuweiten und sein Streben, eigentlicher „Boss“ im Persischen Golf zu werden. London zufolge wollte der Schah zudem die Frage der Abgrenzung der Festlandssockel im südlichen Golf klären, und zwar zu ähnlich vorteilhaften Bedingungen wie gerade für den nördlichen Teil vereinbart. Auch strebe er nach Einfluss auf die Halbinsel und omanische Exklave Musandam, also auf die arabische Gegenseite an der Meerenge von Hormus, und auf das Sultanat von Maskat und Oman im Allgemeinen. ${ }^{87}$

Die USA vermieden öffentliche Stellungnahmen hinsichtlich der bevorzugten Sicherheitsarrangements im Golf. Ein unvorsichtiges Interview des Under Secretary of State

\footnotetext{
${ }^{84}$ Vgl. FRUS 64-68, S. 315 n3; Tehran \#5964, 3.8.68, ,Iran, Volume 2, 1/66-1/69‘, NSF, Country File, Box 136, LBJL; die unklare Abgrenzung hatte immer wieder zu Konflikten wie z.B. der Besetzung von Bohrinseln der ARAMCO durch die Iraner geführt. Vgl. Cramer Jr. Memo, 1.2.68, DDRS: CK3100001065; mit der Abgrenzung der Sockel war aber zugleich ein britisch geplantes "Gesamtpaket" gescheitert, das zugleich eine umfassende Lösung der umstrittenen Inselfrage angestrebt hatte. Vgl. Tehran \#5098, 3.6.68, POL 33 PERSIAN GULF, CF 6769.

${ }^{85}$ Vgl. State \#131326, 16.3.68, FRUS 64-68, XXII, S. 482ff.; Alvandis Interpretation, die US-Politik habe sich deckungsgleich der britischen Position verschrieben, sodass beide Positionen „virtually indistinguishable“ geworden seien, wird aber nicht von den Quellen gedeckt. Man interpretierte die britische Haltung als einseitig die Position der Golfaraber bevorzugend und weigerte sich, Druck auf Teheran zugunsten eines Kompromisses auszuüben. Vgl. Tehran \#5967, 5.8.68, ,Iran Cables, Vol. 2 [1 of 2]', NSF, Country File, Box 136, LBJL; Crawford Despatch to Brown, 27.1.68; Roberts Minute to Brown, 28.3.68, BDEE A, 5, I, S. 140-5, 420f.; Foreign Office TELNO 563 and TELNO 596 to Tehran, 12.3. und 15.3.68, PREM 13/2169, TNA:PRO; vgl. AlvANDI, Origins of Primacy, S. 343; siehe auch ders., Bahrain Question; die Bezeichnung als Federation of Arab Amirates entspricht der US-Sprachregelung. Von britischer Seite waren eher Union of Arab Emirates bzw. United Arab Emirates geläufig.

${ }^{86} \mathrm{Vgl}$. Foster Memo, 21.5.68, FRUS 64-68, XXII, S. $513 \mathrm{f}$.

${ }^{87}$ Vgl. MemCon, 11.3.69, FRUS 69-76, XXIV, S. 226-38.
} 
Eugene Rostow im Januar 1968 schien amerikanische Unterstützung für eine regionale Sicherheitsarchitektur unter Einschluss der Anrainer und möglicherweise auch Pakistans und der Türkei anzudeuten und hatte umgehend zu öffentlichen Dementi der betroffenen Staaten geführt. Im Anschluss vermied man peinlichst jede Erwähnung eines formellen sicherheitspolitischen Zusammenschlusses in der Region. ${ }^{88}$ Auch während des nächsten SchahBesuchs in den USA im Juni 1968 vermied man ostentativ jede Parteinahme - wie auch frühere Besuche so war auch dieser in erster Linie von der Frage weiterer Rüstungskäufe bestimmt. ${ }^{89}$ Mit Bezug auf die künftige Golfordnung ließ in den Gesprächen nur das Interesse Mohammad Rezās an der bestmöglichen militärischen Kontrolle der Straße von Hormus aufhorchen. Eine von Präsident Johnson zugesagte militärische Studie der Frage wurde unter größter Geheimhaltung erstellt, offenbar in erster Linie aus der Sorge über weitreichende iranische Ambitionen und die mögliche Infragestellung des ,international character of the Persian Gulf.“90

Trotz dem offenkundig bestehenden Unbehagen angesichts hegemonialer Ambitionen Irans hielt man an der eigenen formellen Position der Nichteinmischung bzw. der Unterstützung des Aufbaus kooperativer Beziehungen zwischen den Anrainern fest. Das ebenfalls zuvor festgelegte Ziel, einen Rüstungswettlauf in der Region zu verhindern, wurde allerdings nicht ernsthaft verfolgt. Die bereits erwähnte Verdoppelung der Militärkredite für Iran 1968 und die Zusage weiterer \$500 Millionen für die kommenden fünf Jahre demonstrierten mit Nachdruck, dass Rüstungsbegrenzungen keine ernsthaft erwogene Option aus amerikanischer Sicht waren. ${ }^{91}$ Die sich in der jüngsten Forschung etablierende Sichtweise, die USA hätten schrittweise die Idee einer kooperativen Friedenssicherung durch die Anrainerstaaten aufgegeben und ein ,iranisches Primat“ (Roham Alvandi) im Golf akzeptiert, ist nicht zuletzt eine Widerspiegelung des Widerspruchs zwischen den amerikanischen Strategiepapieren zur zukünftigen Ordnung der Golfregion einerseits und der amerikanischen Einwilligung in die Aufrüstung Irans andererseits. In der Tat war auch für die zeitgenössischen

\footnotetext{
${ }^{88} \mathrm{Zu}$ Gene Rostows Interview vgl. Administrative History/Persian Gulf, S. 8f.; ALVANDI, Origins of Primacy, S. 342f.; Saunders warnte im Anschluss vor einer Festlegung: ,The fact is that we have no intention of participating and want to make this clear. Any equation of Persian Gulf and security arrangements in Southeast Asia will do more harm than good, although we obviously want the nations of the Persian Gulf to unite on their own initiative in a variety of ways to ward off Soviet penetration."; Saunders Memo, 2.2.68, FRUS 64-68, XXI, S. 276.

${ }^{89}$ Der Besuch fand direkt im Anschluss zur Entscheidung der Johnson-Administration statt, die Höhe der Iran zugesagten Militärkredite zu verdoppeln. Siehe oben. Vgl. Memo for the Record, 14.6.68, FRUS 64-68, XXII, S 531f.; offenbar gibt es keine Aufzeichnung über den gesamten Inhalt der Gespräche zwischen dem Schah und Johnson am 12. Juni. Vgl. ebd., S. 531 n2; für die Ergebnisse des Besuchs im Allgemeinen vgl. State \#209512, 26.7.68, ebd., S. 546-9.

${ }_{90}$ McClelland Memo, 28.8.68, FRUS 64-68, XXI, S. 312; man fürchtete offenbar auch politische Folgen einer iranischen Option auf Schließung der Meerenge: „Iranian control of the Strait of Hormuz will not in itself keep the peace in the Gulf or maintain its Western orientation."; JCS Memo, 25.6.68, FRUS 64-68, XXII, S. 534; Background Paper, , What is the best military way of dominating the entrance to the Persian Gulf', o.D., ,Iran, 7/1/68-10/31/68', NSF, SHSC, Box 25, LBJL; vgl. MemCon, 29.8.68, POL 33 PERSIAN GULF, CF 67-69; zur Meerenge vgl. RAMAZANI, Strait of Hormuz.

${ }^{91}$ Verschiedene Studien zu ,FY 1970 Military Credit Sales to Iran', o.D., ,Iran, Filed by LBJ Library', NSF, Country File, Box 138, LBJL; ALvand, Origins of Primacy, S. 345f., missversteht die Aufstockung der Militärkredite und geht fälschlich von einer kontinuierlichen US-Politik , to temper Iranian military spending “ aus, erst revidiert durch den „Blankoscheck“.
} 
Entscheidungsträger offenkundig, dass der Abzug britischer Macht aus dem Persischen Golf und der Verzicht auf eine eigene Übernahme der Rolle als „Pacifier“ zwangsläufig zu einer herausgehobenen Position Irans, der wirtschaftlich, demographisch und militärisch stärksten Macht in der Region, führen werde. Assistant Secretary of Defense Paul Warnke gestand dies im Sommer 1968 ein: ,[...] we are placing our money on a modern Persian Emperor to keep open the Persian Gulf.“ 92 In Anerkennung dieser Rolle solle man daher im Golf nur Iran und Saudi-Arabien Waffen verkaufen. Dass dies der Preis für amerikanisches Nicht-Engagement sein werde, war somit auch schon den Akteuren der Johnson-Administration bewusst. Allerdings war mit dieser Politik keineswegs intendiert, eventuelle Ambitionen Irans zur Übernahme einer prädominanten regionalen Führungsrolle - und damit zwangsläufig auch den Ausschluss des strategischen Einflusses der USA im Golf - zu akzeptieren. Die USA beabsichtigen zu keinem Zeitpunkt, ein wie auch immer geartetes iranisches „Primat“ im Golf zu tolerieren. Under Secretary Eugene Rostow brachte die US-Position in einem Gespräch mit Vertretern der Ölindustrie auf den Punkt. Man anerkenne,

[...] that Iran is the strongest country in the Gulf, is moving ahead, and is interested in maintaining its momentum. The USG \{US government; R.P. $\}$ must rely on Iran, not in any way at the expense of Saudi Arabia, but a cooperative relation between Iran and Saudi Arabia was necessarily the keystone of United States plans and hopes for the area. ${ }^{93}$

Im Kern - und hier widerspricht die vorliegende Studie der jüngeren Forschung - hielt auch die Nixon-Administration an dieser Grundmaxime amerikanischer Golfpolitik fest. Somit überwogen nicht nur im Feld der Rüstungsverkäufe, sondern auch im Bereich der Ordnungspolitik der USA in der Region, die Kontinuitäten zwischen beiden Administrationen. Dies mag angesichts des ideologisch-konzeptionellen Gegensatzes zwischen demokratischer und republikanischer Administration und mehr noch angesichts der sehr idiosynkratischen Sichtweise Nixons wie auch seines Sicherheitsberaters Kissingers überraschen.

Die Formulierung der Golfpolitik der neuen US-Administration ging allerdings äußerst schleppend voran. Ein Grund für die Verzögerungen waren eine Reorganisation der Regierungsbürokratie nach den Vorstellungen Kissingers, insbesondere des NSC-Systems, vor allem darauf gerichtet, das Weiße Haus als Entscheidungszentrum nachdrücklich zu stärken. Die verbliebenen informellen Formate wie der „Tuesday Lunch“ wurden abgeschafft. Dasselbe Schicksal erlitten die Senior Interdepartmental Group (SIG) und die untergeordneten regionalen Interdepartmental Regional Groups (IRG) - dafür wurden Elemente eingeführt, die

\footnotetext{
${ }^{92}$ Warnke Memo, 12.6.68, FRUS 64-68, XXI, S. 297: „While it is quite possible that Iran's ,peacekeeping' in the Persian Gulf may become from time-to-time a rather messy operation, I doubt that a large role by the United States would be any more effective--or less messy."; schon während des ersten offiziellen USA-Besuchs des Schahs seit 1964 im August 1967 hatte sich eine Politik nach den Grundzügen der späteren Nixon-Doktrin angedeutet. Vgl. Saunders Memo, 18.8.67, FRUS 64-68, XXII, S. 412f.

${ }_{93}$ MemCon, 6.3.68, FRUS 64-68, XXXIV, S. 403; ähnlich auch sein Kommentar wenige Tage später, ebenfalls im Gespräch mit Ölindustriellen. Vgl. State \#138990, 30.3.68, FRUS 64-68, XXII, S. $493 \mathrm{ff}$, hier 493.
} 
dem alten Eisenhower-System glichen, in der Umsetzung dann aber doch anders gehandhabt wurden. ${ }^{94}$ Noch wichtiger für die Verzögerungen aber war die in der Anfangsphase nicht gegebene Aufmerksamkeit für Golfangelegenheiten, was angesichts der weiterhin alles beherrschenden Vietnam-Frage bzw. der Eskalationen im arabisch-israelischen Verhältnis kaum überraschen dürfte. ${ }^{95}$ Erst im Juli 1969 forderten Kissinger und Nixon von der neuen NSC Interdepartmental Group for Near East and South Asia eine Studie über die Folgen des britischen Abzugs und Empfehlungen für die zukünftige US-Strategie ein. Die mit dem National Security Study Memorandum [NSSM] N66 in Auftrag gegebene Studie sollte bis Ende September 1969 vorliegen, wurde aber erst im Juni 1970 fertiggestellt, ein weiterer Beleg für die untergeordnete Bedeutung dieser Fragen aus Sicht der Nixon-Administration. ${ }^{96}$

Die Studie in Reaktion auf NSSM N ${ }^{\circ} 66$ betonte die strategischen Interessen der USA in der Golfregion, insbesondere die Abhängigkeit der wichtigsten globalen Verbündeten, Westeuropa und Japan, von der dortigen Erdölförderung (55\% und 90\% respektive). Zudem stammten 85\% des von den US-Streitkräften in Indochina verbrauchten Öls ebenfalls aus dem Persischen Golf. Überflug- und Landerechte in Saudi-Arabien und Iran sicherten einen strategisch äußerst bedeutsamen Luftkorridor nach Süd- und Südostasien. Amerikanischer Einfluss, so die Studie explizit, basierten auf den ,freundlichen Beziehungen“ mit diesen beiden Staaten („Our friendly relations with Iran and Saudi Arabia are the mainstay of our influence in the area."). Somit war die zukünftige Aufrechterhaltung der Stabilität und die Sicherung des amerikanischen Zugangs

${ }_{94}$ Die IRGs wurden u.a. ersetzt durch Interdepartmental Groups innerhalb des NSC-Systems. Vgl. PRADOS, Keepers of the Keys, S. 261-8, 311-3; vgl. auch für die interne Reorganisation DAALDER/DESTLER, Shadow of the Oval Office, S. 57-73; DAIGLE, Limits of Détente, S. 31-7; vgl. Kissinger Memo, 7.1.69; NSDM N², 20.1.69, FRUS 69-76, II, S. 11-4, 30-3.

${ }_{95}$ Vgl. FAIN, Ascendance and Retreat, S. 181-8; die Dringlichkeit wurde zusätzlich reduziert durch die sich in den letzten Jahren der Johnson-Administration etablierende Sichtweise einer abnehmenden strategischen Bedeutung der Nahostregion. Die mit der Holmes-Studie (vgl. oben S. 569f., Anm. 26) im Juli 1967 einsetzende interne Debatte über ein wünschenswertes „Disengagement" von der Nahostregion setzte sich bis in das erste Jahr der Nixon-Administration fort. Eine noch von der IRG/NEA direkt gegen die Holmes-Thesen entworfene umfassende Studie wurde Ende Januar 1969 vorgelegt. Die US-Interessen in der Region wurden nunmehr als eher begrenzt definiert, die sowjetische Bedrohung als überschaubar eingeschätzt. IRG/NEA Paper, Basic US Interests in the Middle East', 30.1.69, FRUS 69-76, XXIV, S. 2-9; ähnliche Argumente finden sich auch noch in NSC Paper, ,A U.S. Approach to the Greater Mediterranean Region', 12.6.70, ebd., S. 81-8; IRG/NEA hatte bereits 1967 für eine Kehrtwende bez. der Waffenexporte in die Region plädiert. Vgl. IRG/NEA Study, 14.11.67, encl. to Hartman Memo, 15.11.67, ,SIG/MEMO 33‘, Lot 70 D 263, SIG Files, 1966-1969, Box 2; die oppositionellen Republikaner nutzten die Holmes-Studie im Präsidentschaftswahlkampf von 1968 als Grundlage für eine Grundsatzkritik an der bisherigen Nahostpolitik. Vgl. Saunders Memo, 3.4.68; ,Continuing Crisis in the Middle East“, adopted by The Republican Coordinating Committee, 19.3.68, ,Middle East, Vol. 2 [2 of 2]; 6/65-3/68', NSF, Country File, Middle East, Box 104, LBJL.

${ }^{96}$ Vgl. Kissinger Memo, ,Policy Toward the Persian Gulf ${ }^{*}$ [NSSM N66], 12.7.69, ,NSSM 66‘, NSSM Files, Box 7, RG 273, NACP; die Studie sollte auch die Frage der zukünftigen amerikanischen Marinepräsenz im Golf neu diskutieren. Vgl. Packard Letter, 14.4.70, FRUS 69-76, E-4, doc. 60; die ersten Entwürfe von NSSM Nº6 schienen die herausragende zukünftige Bedeutung Irans zu bestätigen. In einer Diskussion über das weitere Vorgehen verwies Under Secretary of State Eliot Richardson darauf, dass ,a reading of NSSM-66 clearly points in the direction of the conclusion that we have no real option but to bank on Iran."; vgl. MemCon, 14.4.70, ebd., doc. 61; Saunders vom NSC verwies ebenfalls auf die normative Kraft des Faktischen: „It is difficult to conceive that we will reduce our present military relationship with Iran and that we will build our posture in the Persian Gulf around it.“; Saunders Memo, 15.4.70, ,Iran, Vol. I, [...]70‘, NSCF, Country Files-Middle East, Box 601; ein weiterer Grund für die Verzögerung waren offenbar die Unzufriedenheit des NSC mit den ersten Entwürfen der Studie (,,a disaster“). Vgl. Rodman Memo, 23.10.69, ,Persian Gulf - Drafts“, NSCF, HAK Office Files, HAK Administrative and Staff Files, Box 16, RMNL; Rodman Memo, 31.12.69, FRUS 69-76, XXIV, S. 242-5; eine Zwischenfassung in Report, ,Future U.S. Policy in the Persian Gulf', encl. to Davis Memo u.a., 12.3.70, DDRS: CK3100649896. 
eine zentrale strategische Frage für die globalen US-Interessen. Eine Beibehaltung des Pax Britannica wurde als unrealistisch ausgeschlossen, ebenso ein Neutralisierung in Absprache mit Moskau und auch ein eigener vollständiger Rückzug von der Region. ${ }^{97}$ Die Studie definierte daher sechs Optionen für die zukünftige amerikanische Golfpolitik:

(1) Übernahme der bisherigen britischen Rolle als Ordnungsmacht (protector)

(2) Unterstützung eines auserwählten Vertreters, eines ,chosen instrument“ (entweder (a) Iran oder (b) Saudi-Arabien)

(3) Fortsetzung der bisherigen Politik der Förderung iranisch-saudi-arabischer Kooperation

(4) Aufbau enger bilateraler Beziehungen und amerikanischer Präsenz in den kleineren Golfstaaten

(5) Fortsetzung des bisherigen indirekten Verhältnisses zu den kleinen Staaten

(6) Förderung eines regionalen Sicherheitspakts bestehend aus Iran, Saudi-Arabien, Kuwait und den kleinen Golfstaaten ${ }^{98}$

Die Studie sprach sich explizit für Option (3) und damit eine Fortsetzung der Johnson-Position aus: „There is no reason for us to want to choose sides, unless forced to by a crisis. "99 Zugleich gestand man sich aber ein, dass die eigene Politik angesichts der fast bedingungslosen Hinnahme der iranischen Aufrüstungswünsche in der jüngeren Vergangenheit Elemente der Option (2)(a) enthalte. ${ }^{100}$ Dabei darf allerdings nicht übersehen werden, dass die militärische Präponderanz Irans gegenüber Saudi-Arabien auch ohne die exorbitanten USWaffenlieferungen gegeben war und sich ohnehin und zwangsläufig nach einem Abzug der britischen Ordnungsmacht stärker ausgewirkt hätte. Entscheidend für das angemessene Verständnis der US-Strategie ist die Tatsache, dass die USA eben nicht die Gestaltung der zukünftigen Sicherheitsordnung im Golf an Iran übertrugen, sondern durch Signalisierung ihres fortgesetzten Interesses, durch die nun beschlossene Beibehaltung der Flottenpräsenz mittels MIDEASTFOR und durch den Ausbau der parallelen special relationship mit Saudi-Arabien, den iranischen Ambitionen klare Grenzen setzten, dabei aber zugleich Sorge trugen, eine direkte Konfrontation mit Teheran zu vermeiden. Der NSC-Stab verdeutlichte in einem Memorandum an Kissinger den eingeschlagenen Kurs: „The logical strategy lies in marrying

\footnotetext{
${ }^{97}$ Vgl. NSC Paper, ,Persian Gulf: Analytical Summary of IG Response to NSSM 66‘, 4.6.70, FRUS 69-76, XXIV, S. 256-64 (Zitat auf 257); interessant ist der Ausschluss einer Position als ,offshore balancer“ (S. 260), im Grunde die Kernposition bei einer ernsthaften Umsetzung der Nixon-Doktrin, zumindest gemäß ihrer öffentlichen Definition.

${ }^{98}$ NSC Paper, vorherig. Anm., S. 260.

${ }^{99}$ Ebd., S. 261; interessant ist die Begründung der Studie gegen die Unterstützung eines „,chosen instrument“ (,Operationally, we would favor our 'chosen instrument' with military assistance, with support in Consortium negotiations for oil revenues, and with support in territorial disputes in the Gulf."; ebd.) im Golf. Unterstützung für den ohnehin stärksten Staat Iran hätte eine Entfremdung der Saudis und Widerstand der radikalen Araber zur Folge und führe somit zu einer Destabilisierung. Die Studie gestand ein, dass die bisherige Politik de facto Irans Ambitionen bevorzuge: „In short, there are strong elements of this in what we are already doing, though we have not had to choose Iran to the exclusion of Saudi Arabia." (ebd.).

${ }^{100}$ Hier dominierte die normative Kraft des Faktischen: „Options 2(a) and 3 are not mutually exclusive: There is no reason to back Iran and not use our influence to encourage Saudi-Iranian cooperation. There is no feasible way to promote cooperation without recognizing Iran's physical preponderance.“; ebd., S. 262.
} 
what is already in fact extensive support for Iran as the unquestioned power in the area with the logic of cooperation between a strong Iran and a weak Saudi Arabia."101 In der Umsetzung bedeutete dies zuerst einmal eine fortgesetzte Unterstützung für saudisch-iranische Kooperation im Golf, aber zugleich auch eine Fortsetzung einer „fullest feasible U.S.-Iranian relationship“ angesichts der nicht zu bestreitenden Tatsache, dass Iran nunmehr ,the real power in the Gulf" war. ${ }^{102}$ Henry Kissinger und die NSC Review Group schlossen sich der Sichtweise der Studie an und unterstützten ebenfalls Option (3), gemeinsam mit den Optionen (2)(a), Anbindung an Iran, und Option (4), einem begrenzten (nicht-militärischen) Aufbau der US-Präsenz im Golf durch Vertiefung der eigenen Beziehungen mit den kleinen Golfstaaten. ${ }^{103}$ Im November 1970 wurde die zukünftige Golfstrategie der USA mit dem National Security Decision Memorandum (NSDM) $\mathrm{N}^{\circ} 92$ gemäß diesen Überlegungen festgelegt. ${ }^{104}$

Sicherheitsberater Kissinger fasste den Kern der US-Strategie in einem Memorandum für Nixon zusammen:

The logical and obvious strategy is to marry the middle three options: to promote Saudi-Iranian cooperation as the mainstay of a stable regional system, but to recognize Iran's special importance as the preponderant power in the Gulf, and to do what we can to develop a working relationship with the new political entities in the lower Gulf. There is no way to promote

\footnotetext{
${ }^{101}$ Saunders/Kennedy Memo, 3.6.70, ,Persian Gulf-Drafts', NSCF, HAK Office Files, HAK Administrative and Staff Files, Box 16, RMNL; \{Hervorheb. i. Orig.\}; im Anschluss: „We are not likely to diminish our relationship with Iran; we do not want to have to choose between Iran and Saudi Arabia; Saudi-Iranian cooperation is the optimum. That means that the real choice is not really a choice as long as there is no trouble and Saudi-Iranian cooperation continues to grow."

${ }_{102}$ Ebd.; die militärische Zusammenarbeit war somit nicht in erster Linie auf Präponderanz Irans, sondern auf Ausbau der engen bilateralen Beziehungen gerichtet, auch für den Fall eines Scheiterns des kooperativen Ansatzes: ,In arriving at that working view of our strategy, we should avoid relaxing and pinning all our hopes on SaudiIranian cooperation on Iran. Cooperation may break down, and Iran may prove a heavy-handed peace-maker."; vgl. auch Tehran \#418, 4.2.70, POL 33 PERSIAN GULF, CF 70-73; Nixon hatte eine Analyse erbeten,,just how far the U.S. could go in leaving it to Iran to guarantee stability in the Persian Gulf."; die tatsächlichen Erfolgsaussichten einer iranischen Ordnungspolitik wurden als eher begrenzt eingeschätzt. Vgl. Kissinger Memo, 23.6.70, ,Iran, Vol. II[....770‘, NSCF, Country Files-Middle East, Box 601, RMNL.

${ }^{103}$ Vgl. Review Group Meeting Minutes, 5.6.70, FRUS 69-76, XXIV, S. 265-9; tatsächlich tendierte Kissinger selbst anfangs eher zu einer Übernahme der Rolle als Ordnungsmacht durch die USA selbst. Bei der ersten Lektüre der Antwort auf NSSM N 66 hatte er die Optionen (1) und (4) angekreuzt. Vgl. NSC Paper, ,Persian Gulf: Analytical Summary of IG Response to NSSM 66', 4.6.70, ebd., S. $260 n 4, n 5$.

${ }^{104}$ Vgl. NSDM N ${ }^{\circ} 92$, ,U.S. Policy Toward the Persian Gulf', 7.11.70, ohne Folder, NSDM Files, Box 1, RG 273, NACP; die Kernstrategie umfasste die oben genannten Optionen (3) und (4) mit Elementen von (2)(a): ,Approved a general strategy for the near term of promoting cooperation between Iran and Saudi Arabia as the desirable basis for maintaining stability in the Persian Gulf while recognizing the preponderance of Iranian power and developing a direct U.S. relationship with the separate political entities of the area."; zudem verfügte die Direktive das Festhalten an der Flottenpräsenz durch MIDEASTFOR und eine Überprüfung von US-Rüstungslieferungen an die kleineren Golfstaaten. Präsident Nixon hoffte offenbar auf eine bedeutsamere Rolle für Iran im Persischen Golf, wohl stark beeinflusst durch seine persönliche Nähe zum iranischen Monarchen: „If he could do it, it'd be wonderful because he's our friend.“; Mohammad Rezā sei zudem „,awfully good“ bezüglich der Frage Israels. Außerdem leite er ,a virtual dictatorship in a benign way“. MemCon, 8.4.71, FRUS 69-76, E-4, doc. 122; aus quellenkritischer Perspektive ist interessant, wie Nixons exzentrische Ausführungen im internen Gesprächsprotokoll desselben Treffens abgeschwächt wurden. Vgl. Haig Memo to President's File, 8.4.71, ,Iran, Vol. III, 1 Jan-31 Aug 71', NSCF, Country Files-Middle East, Box 602, RMNL; vgl. Kissinger Memo, 25.6.70, ebd., doc. 75; offenbar stand die MIDEASTFOR-Entscheidung auch im Zusammenhang mit der strategischen Aufwertung des Indischen Ozeans im Kontext des Kalten Krieges. Vgl. Sisco Memo, 7.12.70, ,Indian Ocean 1970‘, Lot 71 D 384, NEA/Deputy Assistant Secretary, Rodger P. Davies Subject Files, Box 1; vgl. NSSM N 104, ,Soviet and Friendly Naval Involvement in the Indian Ocean Area, 1971-1975', 9.11.70, FRUS 69-76, XXIV, 132f., zur Ausformulierung der US-Golfpolitik vgl. auch die Memoiren des ehemaligen Offiziellen NOYES, Clouded Lens, 1982², S. 54-111.
} 
cooperation without recognizing Iran's preponderance (else we would lose our influence with Iran); there is no reason to back Iran and not use our influence to encourage Saudi-Iranian cooperation; there is no reason not to develop ties with the sheikhdoms. ${ }^{105}$

Ziel war die Errichtung eines, „self-regulating regional system as capable as possible by itself of filling whatever gap is created by revision of the British protectorate ". ${ }^{106}$ Dies entsprach im Kern der Politik der Johnson-Administration in ihrem letzten Jahr. Im Gegensatz zur jüngeren Forschung, die in NSDM $\mathrm{N}^{\circ} 92$ einen Wendepunkt von einer Gleichgewichtspolitik hin zu einer Akzeptanz eines iranischen Primats sieht ${ }^{107}$, interpretiert die vorliegende Studie die mit NSDM $\mathrm{N}^{\circ} 92$ eingeleitete US-Politik dagegen als Fortsetzung der bisher verfolgten Strategie in Kombination mit einer, wenn auch zu diesem Zeitpunkt noch stark unterbetonten Übernahme der bisherigen Rolle Großbritanniens als Garanten der formellen Unabhängigkeit der kleineren Golfstaaten Kuwait, Katar, Bahrain und den Vereinigten Arabischen Emiraten (VAE), die sich nach dem britischen Abzug anstelle der ursprünglich geplanten Föderation aus neun Emiraten konstituieren sollten. ${ }^{108}$ Letztere Garantie aber basierte nicht auf formellen Beistandsverträgen nach britischem Vorbild bzw. tatsächlicher Truppenpräsenz zu Verteidigungszwecken, sondern war eher implizit - die amerikanische Handhabung der Rolle als ,,regional peacetime stabilizer“ bzw. „Pacifier" unterschied sich hier maßgeblich von der tradierten Hegemonie Großbritanniens im Golf, zumal sie nicht auf kolonialer Vergangenheit aufbaute. ${ }^{109}$

Diese Interpretation hilft zudem das Verhalten Mohammad Rezās im Kontext des britischen Abzugs zu verstehen. Trotz der fortgesetzten Beteuerungen beider Seiten über die beispiellose Qualität des bilateralen Verhältnisses - im Frühling 1969 sprach der Schah von den USA und Iran als ,natural allies“ und nach dem Nixon-Besuch charakterisierte er die Beziehungen als

\footnotetext{
105 Kissinger Memo, 22.10.70, FRUS 69-76, E-4, doc. 91.

${ }^{106}$ Ebd. \{Hervorheb. v. Verf.\}; „In such a system, stable relationships would exist at each level - an equilibrium among the small sheikhdoms of the lower Gulf, collaboration between the larger Gulf states (Iran, Saudi Arabia, Kuwait), and mutual deterrence between the outside powers in the background (U.S., U.K., and USSR)."

${ }^{107}$ So Alvand, Origins of Primacy, S. 361; MCGLINCHEY, Road to Tehran, S. 848.

108 Bahrain und Katar hatten sich mit der Erklärung ihrer Unabhängigkeit im August 1971 aus dem Föderationsprojekt verabschiedet. Die nunmehr nur aus sechs Emiraten bestehenden VAE erlangten im Dezember 1971 ihre Unabhängigkeit. Ra's al-Hajmah trat im Februar 1972 ebenfalls den VAE bei. Vgl. SMITH, Revival and Fall, S. 78-108.

${ }^{109}$ Eine deckungsgleiche Umsetzung der Nixon-Doktrin hätte eine konsequente Offshore Balancer-Position für die USA intendiert. Somit hätte man ausschließlich aus kontra-hegemonialen Ambitionen heraus in die innere Ordnung der Region eingegriffen, also bei sich abzeichnender Etablierung eines „Öl-Hegemons“ im Golf. Ansonsten hätte man sich auf das regionale Gleichgewicht als Garant für die Sicherung der eigenen strategischen Interessen verlassen. Tatsächlich aber sah man durchaus die Möglichkeit eigenen „Managements“ intra-regionaler Zwistigkeiten, also eine eigene Pacifier-Rolle.: „Iran is determined to step into Britain's shoes as the dominating and protecting power in the Gulf. The Arabs do not relish this concept, and there may be a necessity for the U.S. to restrain the Shah."; vgl. Kissinger Memo, 22.10.70, FRUS 69-76, E-4, doc. 91; die indirekte Garantie ergab sich letztlich auch aus dem Festhalten an der eigenen Flottenpräsenz mittels MIDEASTFOR - Kissinger sah sie als Nukleus eines zukünftig notwendig werdenden Stützpunktes, ein weiterer Hinweis auf das Selbstverständnis als eigentlicher Pacifier. Die seitens der jüngeren Forschung betonte Tatsache, dass allein die Machtressourcen Irans zu einer Rolle als „Pillar" befähigten, sind somit gegenstandslos. Die CIA warnte implizit vor einer Aufgabe des kooperativen Ansatzes. Ein direkter Wettbewerb um die Vormacht im Golf zwischen Saudi-Arabien und Iran könne leicht in ,,competitive interference in the lesser states“ ausarten. CIA Memo, ,The Persian Gulf: Groping Toward a New Power Balance', 12.12.69, FRUS 69-76, XXIV, S. 240f., hier 241; vgl. NoYES, Clouded Lens, $1982^{2}$, S. $55,58 \mathrm{ff}$.
} 
„besser denn je“110 - entstanden aufgrund der aus iranischer Sicht ungenügenden USUnterstützung für die eigenen regionalen Ambitionen Spannungen. Die anfängliche Obstruktionshaltung der Iraner im Laufe des Jahres 1968 hinsichtlich einer zukünftigen Kooperation mit Saudi-Arabien und gerade auch der Bahrain-Frage und der politischen Zukunft der anderen britischen Protektorate, bereits oben erwähnt, war Ausdruck dieser Ambitionen und der Frustrationen über die betont äquidistante Haltung Washingtons. Zudem wandte sich Mohammad Rezā früh gegen einen Fortbestand der amerikanischen Flottenpräsenz durch MIDEASTFOR im Golf. Keine andere extra-regionale Macht solle nach dem britischen Abzug im Golf präsent sein, betonte der Schah wiederholt in Interviews: ,[...] we declared long ago that we should not like to see a foreign power in the Persian Gulf. Whether that power be Britain, the United States, the Soviet Union or China our policy has not changed."111 Angesichts der amerikanischen Absicht, die britische Flottenbasis in Bahrain zu übernehmen und MIDEASTFOR fortzuführen, ordnete der Schah Anfang 1972 eine Pressekampagne an, im Rahmen derer ,imperialistische Pläne“ auswärtiger Mächte im Golf scharf attackiert wurden. ${ }^{112}$ Letztlich aber arrangierte sich die iranische Führung notgedrungen mit der fortgesetzten USPräsenz in Bahrain, zumal sich der Irak nach dem Beinahe-Krieg von 1969 mit Iran um den Šațț al-'Arab mehr und mehr Moskau zuwandte und ein begrenztes Weiterbestehen amerikanischer Präsenz infolgedessen opportun erschien. ${ }^{113}$

Angesichts der amerikanischen Weigerung, einem vollgültigen iranischen „Primat“ in der Golfregion zuzustimmen, war Mohammad Rezā gezwungen, alternative Methoden zugunsten eigenen Machtzuwachses zu finden. Eine - wenngleich wenig erfolgreiche - Methode war die Propagierung eines intra-regionalen Sicherheitssystems, insbesondere auf Zusammenarbeit Irans mit Saudi-Arabien und Kuwait basierend, selbstverständlich dominiert seitens Teherans. Vorgeblich im Einklang stehend mit amerikanischen Vorgaben nach Kooperation der

\footnotetext{
${ }^{110}$ MemCon, 1.4.69, FRUS 69-76, E-4, doc. 9; ARMISH/MAAG Message, o.D., encl. to Saunders Memo, 12.6.72, ebd., doc. 204.

${ }^{111}$ Zit. aus Shah's Press Interview, January 1972, abgedr. in RAMAZANI, Persian Gulf: Iran's Role, S. 144; vgl. FAIN, Ascendance and Retreat, S. 192, 194f.; vor dem USA-Besuch des Schahs 1969 drängte die US-Botschaft darauf, den Schah von den Vorteilen einer fortgesetzten Präsenz zu überzeugen. Einmal abgezogen, sei eine Rückkehr der US-Präsenz nur schwer zu bewerkstelligen. Vgl. Tehran \#4054, 6.10.69, FRUS 69-76, E-4, doc. 22. ${ }^{112}$ Vgl. State \#187449, 16.11.70; Tehran \#279, 15.1.72, FRUS 69-76, E-4, docs. 98, 160; in Reaktion auf amerikanische Gesprächswünsche erklärte sich der Schah bereit, MIDEASTFOR zu tolerieren, weigerte sich aber zugleich, seine öffentliche Kritik einzustellen. Vgl. Tehran \#446, 22.1.72, ebd., doc. 163; Dhahran \#A-1, 5.1.72, DEF 1 NEAR E, CF 70-73; vgl. PALMER, Guardians, S. 93ff.; auch innenpolitisch gab es Zweifel an der Übernahme der Basis in Bahrain. Senator Fulbright kritisierte den Schritt und die mangelnde Zusammenarbeit mit dem Senat: „It seems to me that there is a great deal more involved here than a simple decision to continue something which has been going on before. Sharing a British base is one thing, but establishing a base of our own is quite another matter. In the past our role in the Persian Gulf has been subsidiary to that of the British. With their departure from the Gulf and with the establishment of a U.S. base, we will immediately become directly involved in all matters affecting the Gulf."; Fulbright Letter, 14.12.71, DEF 1 NEAR EAST, CF 70-73.

${ }^{113} \mathrm{Im}$ Anschluss an den sowjetisch-irakischen Freundschaftsvertrag gab sich der Schah geläutert bezüglich einer fortgesetzten US-Präsenz im Golf. Vgl. MAAG Telegram encl. to Saunders Memo, 12.6.72, FRUS 69-76, E-4, doc. 204; im Dezember 1971 schlossen die USA ein Abkommen mit dem Herrscherhaus von Bahrain ab, das die Übernahme von Teilen des bisher britischen Stützpunktes durch Washington vorsah. Zudem werteten die USA die bisherige Flottille auf, indem sie das bisherige Flaggschiff durch ein moderneres Kriegsschiff ersetzten. Vgl. Kissinger Memo, 13.3.72, FRUS 69-76, XXIV, S. 355-8; Memo, , The U.S. Naval Presence in Bahrain', 15.7.73, DDRS: CK3100612707.
} 
Anrainerstaaten, war der Plan doch allzu offenkundig auf eine Schwächung der engen Beziehungen zwischen Riad und Washington auf dem Sicherheitssektor gerichtet. Wenig überraschend war das saudische Interesse an einem solchen Arrangement begrenzt. ${ }^{114}$

Angesichts der amerikanischen Haltung war eine Neuordnung der Golfregion durch Gewalt bzw. Drohungen keine gangbare Option, zumal in diesem Fall zusätzlich mit arabischer Gegenmachtbildung zu rechnen war. Mohammad Rezā bemühte sich daher um Verhandlungslösungen der offenen Fragen noch vor dem britischen Abzug, auf die Kompromissbereitschaft Londons und auf amerikanische Vermittlung vertrauend. Dies gelang in Fragen der Abgrenzung der Festlandssockel im Golf in einigermaßen ausgeglichenen Vereinbarungen und auch bezüglich der brisanten Bahrain-Frage, die in erster Linie durch „staatsmännisches“ Entgegenkommen des Schahs gelöst werden konnte. Gegen internen Widerstand des strategischen Establishments in Teheran stimmte der Schah nach Verhandlungen mit London einem Vermittlungsgesuch an den Generalsekretär der Vereinten Nationen zu, implizit eine Aufgabe der iranischen Ansprüche. Ein Kommission der UNO stellte den Wunsch der , überwältigenden Mehrheit“ der Bahrainer nach Unabhängigkeit fest der iranische Mağles stimmte der entsprechenden Resolution des UNO-Sicherheitsrates im Mai $1970 \mathrm{zu}^{115}$

Im Gegenzug hatte der Schah auf britisches Entgegenkommen in der Inselfrage gehofft und drohte zudem mit Sabotage der alles andere als etablierten Föderation der Emirate der Vertragsküste. Aus US-Sicht waren die Behauptungen der Iraner über die besondere strategische Bedeutung der drei Inseln nahe Hormus eher fraglich, und über die negativen Folgen einer wahrscheinlicher werdenden militärischen Lösung machte man sich keine Illusionen. Intern sah das Department of State die kompromisslose Haltung Teherans sogar als „,inconsistent with Iran's looming role as primary force for area stability“ an. ${ }^{116}$ Der britischen Diplomatie gelang es, in der Frage der Insel Abu Musā eine gütliche Einigung zwischen dem

\footnotetext{
114 Vgl. ed. note in FRUS 69-76, XXIV, S. 240; seitens der USA hielt man sich bezüglich eines regionalen Sicherheitsarrangements nach den negativen Erfahrungen im Anschluss an das Eugene Rostow-Interview vom Januar 1968 zurück. Vgl. State \#4705, 12.1.70, DEF 1 NEAR E, CF 70-73; demgemäß entzog man sich auch britischen Wünschen nach einer Aufwertung der CENTO-Allianz, die trotz des britischen Abzugs aus dem Persischen Golf weitergeführt wurde. Vgl. State \#178786, 30.10.70, FRUS 69-76, XXIV, S. 95-8; vgl. auch Sober Memo, 10.2.69, CENTO 3, CF 67-69; vgl. Defence Policy Staff Note for COS Committee, 23.9.71, BDEE A, 5, I, S. 460-4; vgl. PoOLE, History of the JCS: X, S. 194f.

${ }^{115}$ Vgl. CC(70)13, 19.3.70, fos 181-182, CAB 128/45, TNA:PRO; Hintergründe bei AlvaNDI, Bahrain Question, S. $174 \mathrm{ff}$.

${ }_{116}$ State \#36768, 3.3.71, FRUS 69-76, E-4, doc. 118; die iranische Haltung in der Inselfrage sei ,,provocative and uncompromising“; Brewer Memo, 27.2.70, ebd., doc. 51; vgl. Tehran \#2506, 13.6.70, DDRS: CK3100675709; im Weißen Haus hoffte man auf einen iranischen Verzicht auf Gewalt: „It would seem that the Shah would want to avoid a precipitous move if he is to play a leading role in the Gulf."; Saunders/Neaher, 19.5.71, FRUS 69-76, XXIV, S. 315; Kissinger vermutete, Mohammad Rezā benutze die Inselfrage, um ein Zustandekommen einer Föderation der Emirate zu verhindern, um letztlich eine iranische „Hegemonie“ über die Vertragsküste zu etablieren. Vgl. MemCon, 25.6.71, ebd., S. 322ff.; in London nahm man die US-Politik der Nicht-Involvierung offenkundig anders war. Eine Aussage Siscos vor dem Kongress, wonach man nach einer Verständigung in der Inselfrage gestrebt habe, kommentierte ein Offizieller scharf: ,Those of us dealing with the affairs of the Gulf at this time would hardly have described the American role in such terms!"; Melhuish Memo, 27.9.72, FCO 8/1806, TNA:PRO.
} 
Emirat Sharjah (Al-Šāriqa) und Teheran herbeizuführen - ähnliche Versuche mit Ra's alHajmah über die Große und die Kleine Tunb-Insel aber scheiterten. Am 30. November 1971 und damit einen Tag vor dem Auslaufen der britischen Beistandsgarantie für die Emirate landeten schließlich iranische Truppen auf den umstrittenen Inseln und besetzten diese. Eine kooperative Regelung der neuen Sicherheitsordnung im Persischen Golf scheiterte somit an der eher untergeordneten Frage der Souveränität über diese Inseln: Die scharfe Reaktion der arabischen Staaten - Irak brach sogar die diplomatischen Beziehungen zu Teheran und London aus Protest ab, Libyen nutzte den Vorwand zur Nationalisierung der britischen Ölinteressen und der Einsatz von Militärgewalt belasteten das arabisch-iranische Verhältnis in den Folgejahren und stellten zudem eine schwerwiegende Hypothek für die Vereinigten Arabischen Emirate in den ersten Jahren ihrer Existenz dar. ${ }^{117}$

\footnotetext{
${ }_{117}$ Sharjah und Iran einigten sich auf geteilte Souveränität für Abu Musā. Die iranischen Truppen wurden nach der Landung von einem Begrüßungskommando des Emirs empfangen. Der Emir von Ra's al-Hajmah hatte seine Polizeikräfte trotz Wissens über die bevorstehende Landung nicht gewarnt - sieben Soldaten kamen ums Leben. Vgl. Eliot Jr. Memo, 1.12.71, FRUS 69-76, XXIV, S. 350f.; Rogers Memo, 16.12.71, FRUS 69-76, E-4, doc. 153; $\mathrm{CM}(70) 8,23.7 .70$, fos 103-104, CAB 128/47; CM(71)56, 18.11.71; CM(71)61, 2.12.71, CAB 128/49, TNA:PRO; vgl. BALFOUR-PAUL, End of Empire, S. 131-4; Kelly, Gulf and the West, S. 92-7; MOBLEY, Tunbs and Abu Musa; die zeitgenössische Literatur aus dem Umfeld der Pahlavists beschrieb die „Lösung“ der Inselfrage als iranischen diplomatischen Erfolg. Vgl. Burrell/CotTrell, Iran, Afghanistan, Pakistan, S. 1ff.; auch die Grenzfrage zwischen den VAE und Saudi-Arabien, in Teilen die alte Buraimi-Frage, blieb vorerst ungelöst. Saudi-Arabien verweigerte daher im Gegensatz zu Iran den VAE vorerst die diplomatische Anerkennung. 1974 einigten sich Saudi-Arabien und die VAE auf einen territorialen Kompromiss. Vgl. CIA, NIE 30-1-71, 1.4.71, FRUS 69-76, XXIV, S. 306-10; wie stark die verfolgte iranische Hegemonie im Persischen Golf mit der Person Mohammad Rezās identifiziert wurde, zeigte sich in der ostentativ anti-imperialistischen Kehrtwende nach der Revolution und der öffentlichen Weigerung der Revolutionsregierung, weiterhin die Rolle eines „Gendarmen“ in der Region spielen zu wollen. Vgl. CIA, National Foreign Assessment Center Assessment, ,New Directions in Iranian Foreign Policy“', 20.3.79, CREST: \#RDP80*001-5.
} 
VIII. 3. Kooperation und Gegensatz: Iranische und amerikanische Ordnungspolitik im Persischen Golf, 1972-1975

„If military power were the sole prerequisite of leadership, Iran could provide a Pax Persica“, kommentierte die CIA im Herbst 1972 nüchtern die sich etablierende Sicherheitsordnung im Persischen Golf. ${ }^{118}$ Territoriale Konflikte und kulturelle Differenzen stellten die iranische Führungsfähigkeit in der Region aber in Frage. Tatsächlich waren die konkreten politischen Vorteile gegenüber den Nachbarn, resultierend aus der militärischen Übermacht Irans, nicht wirklich erkennbar. Der strategische Einfluss Irans in der Golfregion blieb nach dem britischen Abzug begrenzt - Jordanien und Pakistan begannen zudem, den Aufbau militärischer Kapazitäten vorwiegend in Ausbildungsrollen zu unterstützen und stellten somit gangbare, wenngleich geographisch entfernte Alternativen dar. ${ }^{119}$

Saudi-Arabien und die kleineren arabischen Golfstaaten bemühten sich in der Folgezeit intensiv um eigene „Sonderbeziehungen“ zu den USA, um iranischen Druck und Vormachtstreben abzufedern. Iran sei nicht die alleinige Antwort auf die Probleme im Persischen Golf, kommentierte der US-Botschafter in Kuwait angesichts der Entschlossenheit seiner Gastgeber to „go American“. ${ }^{120}$ Im Juni 1972 schlug der saudische Verteidigungsminister Prinz Sultān bei einem Washingtonbesuch die Schaffung einer eigenen saudisch-amerikanischen special relationship gerichtet auf die gemeinsame Verteidigung der Großregion bestehend aus Persischem Golf, der Arabischen Halbinsel und dem Roten Meer vor. Die Saudis plädierten für eine koordinierte Stabilisierungspolitik mit Iran für die kleineren Golfstaaten, damit die Grundidee hinter NSDM N ${ }^{\circ} 92$ selbst aufgreifend. ${ }^{121}$ Ein wichtiger Einflussfaktor war dabei die absehbare Tatsache, dass Saudi-Arabien in der Zukunft genauso viel Öl exportieren würde wie alle anderen Golfstaaten zusammen, Iran eingeschlossen. Die Saudis setzten sich von anderen Förderländer rhetorisch wie sachlich ab und zeigten sich - anders als der zunehmend

\footnotetext{
${ }^{118}$ CIA Memo ,The Persian Gulf: The End of Pax Britannica', 21.9.72, FRUS 69-76, XXIV, S. 382-91, hier 382; vgl. Arthur Despatch to Douglas-Home, 19.4.71, BDEE A, 5, I, S. 443-7.

${ }_{119}$ Vgl. CIA Memo, vorherig. Anm.; ein pakistanischer Offizier kommandierte die neu gegründete Luftwaffe Abu Dhabis, bestehend aus französischen Kampfjets, die ausschließlich von pakistanischen Piloten geflogen wurden. Vgl. Islamabad \#6472, 27.7.72, POL NEAR E-PAK, CF 70-73; vgl. CIA Memo, 23.7.73, ,Shah of Iran (17 July 1973)‘, NSCF, VIP Visits, Box 920, RMNL; CIA Intel Memo, 20.3.70, DDRS: CK3100705121; für den Verbleib britischen Einflusses auch nach dem Abzug vgl. Carrington Minute to Heath, 30.7.71, BDEE A, 5, I, S. 458f.; zu Jordanien vgl. Murray/Tehran to FCO, 22.6.72, fol. 45, FCO 8/1806, TNA:PRO; vgl. Information Nr. 856/75, 6.12.75, BStU, MfS, HV A, Nr. 119.

${ }^{120}$ Kuwait City \#1293, 17.11.71, FRUS 69-76, XXIV, S. 347; ebd., S. 344 n2; die Kuwaitis offerierten den USA nun weitreichende Kooperationen in Wirtschafts- und Sicherheitsbereichen, unter anderem die Entsendung amerikanischer Militärberater. Im Mai 1971 war der Beistandsvertrag mit Großbritannien ersatzlos ausgelaufen. Vgl. Sisco Memo, 22.12.70, DDRS: CK3100633507.

${ }_{121}$ Saudi-Arabien wünschte zudem die Einbeziehung Jordaniens. Vgl. Kissinger Memo, 15.6.72, FRUS 69-76, XXIV, S. $511 \mathrm{ff}$; Kissinger zeigte sich überrascht über den für die Saudis eher ungewöhnlichen Vorstoß und vermutete den Einfluss der US-Ölkonzerne. Angesichts der weiterhin bestehenden militärischen Schwäche sah man (noch) keine saudische Fähigkeit, als „Policeman“ auf der Arabischen Halbinsel zu agieren. Dennoch drängte man auf Ausdehnung des politischen Engagements. Vgl. Sisco Memo, 18.5.72, ebd., S. 504-7; zur Unzufriedenheit mit den Saudis vgl. auch CIA \#WH31815, 30.6.73, CIA-Helms.
} 
arrogant auftretende Iran - gerade in der Anfang der 1970er Jahre virulenten Frage der Beteiligungen der Förderstaaten an den Erdölunternehmen kooperativ, eine in Washington mit großer Erleichterung registrierte Haltung. ${ }^{122}$ Die neue saudische Flexibilität war die Grundlage für eine Aufwertung des bilateralen Verhältnisses zwischen Riad und Washington. Auf USSeite realisierte man rasch, dass Saudi-Arabien angesichts der über drei Jahrzehnte aufgebauten Verbindungen und der fehlenden eigenen Verteidigungsfähigkeit der ideale Partner wäre, wenn man schon eine einseitige Abhängigkeit von einem einzelnen Ölproduzenten in Kauf zu nehmen gezwungen war. ${ }^{123}$ Der saudische Vorschlag ,to create a self-reinforcing link which would guarantee our oil supplies and their security ${ }^{\text {"124 }}$ sollte mittelfristig zur Etablierung einer einzigartigen saudisch-amerikanischen Sonderbeziehung führen, die sich in den darauffolgenden Jahrzehnten bis zur Gegenwart immer weiter vertiefte. Basierend auf einer Matrix aus US-Sicherheitsgarantien, militärischer Präsenz, Rüstungsverkäufen, Ölexporten und saudischen Investitionen im amerikanischen Markt, bildet dieses interdependente Verhältnis eine der Grundachsen der weltpolitischen Ordnung bis weit ins 21. Jahrhundert. Gerade die Investitionen in den USA nahmen bald herausragende Bedeutung an, insbesondere die saudischen Ankäufe amerikanischer Staatsanleihen im Rahmen des Petrodollar Recycling infolge eine Reihe von geheimen Absprachen in der Nachfolge der Ölkrise von $1973 .{ }^{125}$

Es waren gleichermaßen diese Avancen der Golfaraber wie auch die graduelle Neueinschätzung der eigenen strategischen Interessen, die zu einer nun immer mehr auf Balancierung des iranisch-arabischen Verhältnisses in dieser Schlüsselregion abzielenden Interpretation von NSDM N92 vom November 1970 und einer wieder bedeutender werdenden eigenen Rolle führten. NSDM Nº186 vom August 1972 genehmigte nun direkte Rüstungslieferungen an die Staaten im unteren Persischen Golf und an Oman, was zwangsläufig auch hier eine Steigerung

122 So erwarb Saudi-Arabien zum 1. Januar 1973 ein Viertel ARAMCOs und stockte diese „Beteiligung“ schrittweise bis 1980 auf $100 \%$ auf, die de facto-Nationalisierung bei großem eigenen Entgegenkommen durchsetzend. Die saudische Diplomatie setzte diese gemäßigte Ölpolitik und den eigenen Einfluss in der OPEC in den sicherheitspolitischen Verhandlungen mit den USA geschickt ein. 1972 boten die Saudis einen eigenen Beitrag zur langfristigen Energiesicherheit der USA im Gegenzug zu saudischen Großinvestitionen im Downstream-Bereich an und somit die feste Etablierung einer engen wirtschaftlichen Interdependenz zwischen beiden Staaten. Vgl. MemCons, 29.9.72, FRUS 69-76, XXIV, S. 523-7; MemCon, 30.9.72, FRUS 69-76, XXXVI, S. 347-51.

${ }_{123}$ Vgl. Jidda \#3347, 17.10.72, FRUS 69-76, XXIV, S. 529-32.

124 ,The Saudi motives behind this proposal actually seem to be more political than economic in that they would obviously like us to become a powerful protector for them against the Soviets (whom they greatly fear) and other governments in the area who pose a potential threat to them (presumably Iran and Israel as well as radical Arab regimes)."; NSC Staff Briefing Paper, 14.12.72, ebd., S. 539; vgl. auch Saunders/Loken Memo, 10.11.72, ,Saudi [...]Saud (1972)‘, NSCF, Presidential Correspondence, Box 761, RMNL.

725 Zentral für diesen Zusammenhang die sozialwissenschaftliche Arbeit von SPIRO, Hidden Hand; vgl. Sisco Memo, 18.6.69, ,South Arabia 1969‘, Lot 73 D 376, NEA/Deputy Assistant Secretary, Rodger P. Davies Subject Files, Box 1; vgl. ed. note, FRUS 69-76, XXXVI, S. 535ff; die CIA warnte im Frühling 1973 vor den machtstrukturellen Veränderungen, die sich aus dem Anstieg des Ölpreises ergaben: „The trends already in motion, if continued through 1985, would result in the Middle East oil producing states accumulating foreign assets that would be truly astronomical. Their assets would range from a low of $\$ 100$ billion to as much as $\$ 180$ billion by 1985, comparable to total gross U.S. foreign assets and to more than double net U.S. foreign assets."; CIA, Office of Economic Research, ,Middle East: Some Implications of Increasing Oil Revenues', 30.3.73, ebd., S. 444f.; für Saudi-Arabien als einer "major world monetary power", die Sonderbeziehungen notwendig mache, siehe Cooper/Saunders Memo, 5.6.74, ebd., S. 998-1003, hier 1002; vgl. Saunders et al. Memo, 8.5.73, DDRS: CK3100676971; zur besonderen saudischen Rolle als zukünftiger „,swing producer“ vgl. Odeen Memo, 7.9.73, DDRS: CK3100525313. 
des amerikanischen Einflusses mit sich brachte. ${ }^{126}$ Liberalisierung cum Intensivierung der eigenen Waffenexportpolitik gegenüber Saudi-Arabien und den kleineren Golfstaaten waren gewissermaßen ein Ausgleich zu der nun äußerst liberal gehandhabten Aufrüstungspolitik gegenüber Iran. Politisch aber war der Schritt bedeutsam, zeigte er doch im Kern die Ablehnung eines einseitigen iranischen Primats und das weiterhin aktive Management der regionalen Machtbalance durch den tatsächlichen regionalen Hegemon, die Vereinigten Staaten. Ganz im Geiste der in dieser Studie vertretenen Interpretation der Nixon'schen Golfpolitik demonstrieren die deklassifizierten Archivalien auch in der Folgezeit eine abwartende bis ablehnende Haltung gegenüber jeglichen Regungen iranischer Vormachtstrebens. So berichtete der neue US-Botschafter Richard Helms im März 1973 von den wachsenden Spannungen zwischen Iran und seinen Nachbarn: „Arabs feel that Iranians in general and the Shah in particular are so contemptuous of them and are so arrogant in their dealings with them that true cooperation probably is not possible. The Arabs genuinely fear Iranian colonialism in the Gulf.“ Helms empfahl die Annahme einer amerikanischen Rolle als „Pacifier“. ${ }^{127}$ Bereits im Mai 1973 leitete Kissinger eine erneute Revision der US-Golfpolitik ein, ausgelöst durch die Entwicklungen in der Region und insbesondere die rasant steigenden Ölpreise und die daraus resultierenden einschneidenden strategischen Folgen für die USA. NSSM N ${ }^{\circ} 181$ ordnete unter anderem eine Untersuchung des gegenwärtigen Status der iranischsaudischen Kooperation in der Golfregion an und deutete zudem eine weitere Vertiefung der US-Rolle in den kleineren Emiraten an. Interessant ist die nun gleichwertige Behandlung der saudischen mit der iranischen Rolle trotz der weiterhin gegebenen eindeutigen militärischen Überlegenheit Irans. ${ }^{128}$ Der NSC-Stab empfahl eine Aufwertung der Bedeutung SaudiArabiens auf eine Rolle in der regionalen US-Strategie ähnlich der Rollen Israels, Irans und Jordaniens. Weiterhin ungeklärt blieb aber die Aufrechterhaltung einer saudisch-iranischen

\footnotetext{
${ }^{126}$ Vgl. NSDM N ${ }^{\circ} 186$, ,US Military Supply Policy for the Lower Persian Gulf States and Oman', 18.8.72, FRUS 69-76, XXIV, S. 379f.; MCGLINCHEY, Road to Tehran, S. 18f., fehlinterpretiert die Direktive als Vollendung der Blankoscheck-Politik gegenüber Iran. Tatsächlich entsprach die neue Weisung einer Vertiefung der alten Option (4) in NDSM N92; vgl. Elliot/Washington to Parsons, 13.6.72, fol. 41, FCO 8/1806, TNA:PRO.

127 „One of the key roles the US may play in the region is to assure that emotions are kept suppressed and are not permitted to break down the uneasy truce between the two parties."; Kissinger Memo, 24.4.73, ,Iran, Vol. V, May 73-Dec 73،, NSCF, Country Files-Middle East, Box 603, RMNL; das wiedergegebene Zitat in der FRUS-Version ist fehlerhaft und gibt ,genuinely“ mit „,generally“ wieder. Siehe ed. note, FRUS 69-76, XXVII, S. 67; bereits im Januar 1973 hatte die US-Botschaft vorsichtig gewarnt, eine militärisch umgesetzte Ordnungspolitik Irans in der Region könne amerikanische strategische Interessen schädigen. Vgl. Tehran \#A-4, 9.1.73, ebd., S. 1-19; die Senior Review Group kritisierte die Widersprüchlichkeit der eigenen Politik: „The basic issue to be discussed is the assumption which has been at the base of our policy to date - that Iranian-Saudi cooperation is the best guarantee of stability in the Gulf. The issues are whether the Saudis are showing the capacity to hold up their end of the cooperation and whether the relationship between Iran and Saudi Arabia is good enough so that it is realistic to think in terms of such cooperation. The alternative into which we are drifting is to assume that the Iranians will take care of stability in the Gulf."; ed. note, FRUS 69-76, XXVII, S. 67ff., hier 69 \{Hervorheb. i. Orig.\}; vgl. CIA \#sanitized to Kissinger, 31.3.73, CIA-Helms.

${ }^{128}$ Siehe NSSM N 181 , ,U.S. Policy in the Arabian Peninsula and the Persian Gulf‘, 10.5 .73 , ,NSSM 181 ‘, NSSM Files, Box 15; NSSM No181 nimmt direkten Bezug auf die sich aus einer früheren Studie ergebenden strategischen Konsequenzen für die Golfpolitik der USA. Diese frühere Studie zur Energiefrage betonte offenbar die steigende Bedeutung Saudi-Arabiens infolge der Ölsituation und trug zusätzlich zur Einleitung einer noch gleichgewichtigeren Twin Pillars-Politik im Golf bei. Die Studie ist NSSM N¹74, , National Security and U.S. Energy Policy“, 8.3.73, ebd., ,NSSM 174', NSSM Files, Box 14, RG 273, NACP; siehe auch NSSM 174 Report, August 1973, ebd.; vgl. INR Intel Note RNAN-36, 3.8.73, POL IRAN-SAUD, CF 70-73; CIA, NIE 30-1-73, 7.6.73, DDRS: CK3100664014.
} 
Kooperation trotz nicht bestreitbarer militärischer Prädominanz Irans bzw. die unterstützende Rolle der USA hinsichtlich dieser Kooperation, ein Problem, das im Grunde bis zur Iranischen Revolution ungelöst blieb. ${ }^{129}$

Trotz des Ausbleibens ernsthafter Bemühungen hinsichtlich strategischer Kooperation zwischen Riad und Teheran im Persischen Golf und der militärischen Prädominanz Irans kam es dennoch nie zu einer iranischen Abenteuerpolitik entlang der Linien, die Paul Erdman in seinem fiktiven Szenario eines „Ölkriegs von 1976“ entworfen hatte. Eine solche hätte zu einer amerikanisch-iranischen Konfrontation geführt, ein Umstand, der auch Mohammad Rezā bewusst war. Bei aller ostentativ zur Schau gestellten Unabhängigkeit Irans war man sich dennoch der unbestreitbaren Abhängigkeiten vom ehemaligen Patron und jetzigen Alliierten bewusst, vor allem im Bereich moderner Militärtechnologie. Zweifellos aber hoffte Mohammad Rezā auf größere Handlungsfreiheit in der Zukunft im Kontext der Akzeptanz Irans im Kreis der Weltmächte, seiner Stellung als militärischer Vormacht im Golf und wohl auch infolge einer konsequenten Fortsetzung des Nuklearprogramms mit allen seinen militärischen Implikationen. Vorerst aber lenkte der Schah - in seiner Golfpolitik durch oben geschilderte Faktoren eingedämmt - seine außenpolitischen Ambitionen auf die breitere Region. Man vermied von iranischer Seite angesichts des traditionellen arabisch-iranischen Gegensatzes, der eigenen Sonderbeziehungen mit Israel und der Emotionalität des Themas in der arabischen wie in der weiteren islamischen Welt eine Involvierung in den arabisch-israelischen Konflikt. ${ }^{130}$ Allerdings unterstützte Mohammad Rezā den Seitenwechsel Ägyptens im Kalten Krieg hin zum Westen sowohl diplomatisch wie auch mittels Gewährung von generöser Wirtschaftshilfe an Sadat. Seine Unterstützung für König Husseins Position in Jordanien war vorwiegend seiner Sorge vor einem radikalen Dominoeffekt in Saudi-Arabien und Kuwait im Falle dessen Sturzes geschuldet. ${ }^{131}$ Auch die Beziehungen zu den CENTO-Verbündeten und RCD-Partnern Türkei und Pakistan boten wenig Spielraum für eine eigene Großmachtpolitik und bildeten alles andere als einen Schwerpunkt iranischen auswärtigen Handelns in dieser Zeit. ${ }^{132}$

\footnotetext{
${ }^{129}$ Die internen Diskussionen drehten sich um das Dilemma, die Anerkennung der iranischen Prädominanz mit dem Wunsch nach Kooperation mit den Anrainern zu vereinbaren. Siehe ed. Note; Interdepartmental Working Group Paper, 25.4.74, FRUS 69-76, XXVII, S. 67ff., 183-93; Deputy Secretary of Defense William P. Clements warnte explizit vor einer Einbeziehung der Iraner in die amerikanischen Notfallplanungen. Siehe Review Group Meeting, 20.7.73, ebd., S. 70-8; vgl. ebd., S. 75 n6; die Ford-Administration ordnete im Februar 1976 eine erneute Revision der US-Golfpolitik an. Vgl. Scowcroft Memo, ,U.S. Policy Toward the Persian Gulf‘ [NSSM N²38], 13.2.76, ,NSSM 238', NSSM Files, Box 16, RG 273, NACP; Barr Memo, 31.7.76, ,Iran 1975, May-1979 Feb“, Box 83, Haig Papers, LOC; analog zu dieser Interpretation NOYES, Clouded Lens, S. 120ff.; für die nachfolgende US-Politik in der Golfregion vgl. KuPCHAN, Persian Gulf and West, S. 44-98; ACHARYA, U.S. Military Strategy, S. 37-88.

${ }_{130}$ Vgl. RAmAZANI, Iran and the Arab-Israeli Conflict; PARSI, Iran's Arab Option, S. 498-503; zur Zunahme der iranischen Involvierung nach 1974 vgl. aber ed. note, FRUS 69-76, XXVII, S. 285-8.

${ }^{131} \mathrm{Vgl}$. Tehran \#2604, 4.5.72, FRUS 69-76, E-4, doc. 184; vgl. die Einträge vom 4.6.74 und 7.2.76, in: ALAM, Shah and I, S. 374, 468; im Vierten Arabisch-Israelischen Krieg von 1973 unterstützte der Schah Saudi-Arabien mit einer Leihgabe von 5 C-130-Transportflugzeugen. Vgl. CIA \#sanitized, 13.10.73, CIA-Helms.

${ }^{132}$ Vgl. CIA, SNIE 34-70, 3.9.70, FRUS 69-76, E-4, doc. 86; die schwere Krise in den türkisch-amerikanischen Beziehungen Mitte der Dekade im Anschluss an die Zypern-Intervention 1974 nutzte der Schah eher zur Übernahme der Rolle Ankaras als wichtigster regionaler Verbündeter im Gesamtkonstrukt von Washingtons Nahostpolitik. Im Anschluss an die türkische Schließung von US-Militärbasen in Vergeltung für das Waffenembargo des US-Kongresses half der Schah ähnlich wie gegenüber Pakistan 1965 mit der Lieferung von
} 
Mohammad Rezā kompensierte daher die Beschränkungen seiner außenpolitischen Handlungsmöglichkeiten im Golf selbst und in der breiteren Regionalpolitik im Nahen und Mittleren Osten durch eine Fokussierung auf den Indischen Ozean und auf weltpolitische Belange. Einsetzend Ende 1971, begann der Schah eine ambitionierte Strategie im Hinblick auf den Indischen Ozean zu formulieren. Im Gespräch mit dem Iran besuchenden USVizepräsidenten Spiro Agnew nannte der Schah die zunehmenden maritimen Aktivitäten der Sowjets als Begründung für diese neue strategische Schwerpunktsetzung. ${ }^{133}$ Neben der immer willkommenen Nebenerscheinung, dass die Ausweitung der eigenen strategischen Interessen auch immer als Rechtfertigung weiterer Aufrüstung dienen konnten, ergaben sich aus der breiteren Indischer Ozean-Strategie auch andere Vorteile aus Sicht Irans. Zum Einen ermöglichte der Indische Ozean einen Ausbau der eigenen Flotte, bislang vernachlässigt gegenüber den andern Teilstreitkräften, ohne dass dies zwangsläufig als Bedrohung für die anderen Golfanrainerstaaten zu werten war, was aber dennoch die regionale Machtbalance noch weiter zugunsten Irans veränderte. Zum Anderen hätte militärische Kontrolle des Arabischen Meers durch Iran mittelfristig die sowjetischen, aber auch die amerikanischen Kommunikationslinien in den Persischen Golf durchschnitten und jede militärische Machtprojektion von der Zustimmung Teherans abhängig gemacht. Wenn auch militärisch angesichts der dann doch begrenzten Kapazitäten Irans nur schwer umsetzbar, so balancierte die neue maritime Strategie Irans in gewisser Weise sowohl die „Pacifier“-Rolle Washingtons im Golf und stellte zudem ein Gegengewicht zum Nukleus zukünftiger militärischer Machtausstrahlung der USA rund um das bereits mehrfach erwähnte Diego Garcia dar. Nicht zuletzt dieses Anliegen brachte nun auch das selbstbewusste Indien mit Iran zusammen. Tatsächlich sicherte sich Iran Hafenrechte in Mauritius im Gegenzug für großzügige Wirtschaftshilfe - in den Malediven scheiterte ein ähnlicher Vorstoß offenbar am indischen Widerspruch und dem eigenen Hegemonieanspruch Neu-Delhis. Der geplante Ausbau des zuvor peripheren Hafens in Čābahār zu einer riesigen Marinebasis mit kolportierten Kosten von $\$ 600$ Millionen diente der Machtprojektion in den Indischen Ozean und vervollständigte zudem die iranische Kontrolle der Meerenge von Hormus im Zusammenspiel mit den Positionen in

\footnotetext{
dringend benötigten Ersatzteilen für amerikanische Ausrüstung, offenbar mit Duldung der Ford-Administration. Vgl. KAYAOǦLU, Turkish-Iranian Cooperation; die vormals engen Beziehungen zu Islamabad litten bald unter dem indisch-iranischen Rapprochement. Gleichzeitig fürchtete der Schah einen Zusammenbruch des pakistanischen Reststaates nach Abspaltung Bangladeschs infolge des Dritten Indisch-Pakistanischen Krieges von 1971. Vgl. CHUBIN, Iran's Foreign Policy, S. 210f.; CENTO selbst wurde von US-Seite inzwischen als völlig wertlos und als „tiresome charade“ angesehen. Vgl. Miklos Memo, 13.3.72, ,Iran 1972“, Lot 73 D 376 , NEA/Deputy Assistant Secretary, Rodger P. Davies Subject Files, Box 2.

${ }^{133}$ Mohammad Rezā erwähnte Agnew gegenüber den zeitweilig erwogenen Erwerb eines Flugzeugträgers für diese Zwecke, doch habe man sich angesichts der exorbitanten Kosten für eine vorwiegend luftgestützte Strategie zur Kontrolle von Arabischem Meer und angrenzenden Bereichen des Indischen Ozeans entschieden. Dies bildete zugleich die militärische Rechtfertigung für iranische Anfragen bezüglich einer Ausstattung mit amerikanischen Tankflugzeugen. Zudem wäre auf diese Weise die Reichweite iranischer militärischer Machtprojektion erheblich gesteigert worden. Vgl. USDEL Shiraz \#16, 15.10.71, FRUS 69-76, E-4, doc. 149; Eliot Jr. Memo, 14.12.72, ,Iran, Vol. IV, 1 Sep 71-Apr 73‘, NSCF, Country Files-Middle East, Box 602, RMNL; Davies Memo, 8.12.72, ,Iran 1972‘, Lot 73 D 376, NEA/Deputy Assistant Secretary, Rodger P. Davies Subject Files, Box 2.
} 
Bandar 'Abbās und den gerade besetzten Inseln im Golf. ${ }^{134}$ Offenkundig beunruhigt von den iranischen Ambitionen verhinderte die US Navy 1974 den Verkauf dreier atomgetriebener Jagd-U-Boote der Sturgeon-Klasse an die kaiserliche Marine - ein weiterer Beleg für die auch nach dem „Blankoscheck“ bestehenden Einschränkungen. ${ }^{135}$

Noch eindrücklicher, was die iranischen Ambitionen bezüglich einer umfassenden Kontrolle der Meerenge von Hormus anging, war die Entscheidung des Schahs, militärisch in Oman zu intervenieren. Die Herrschaft des Sultans von Oman war seit über einem Jahrzehnt durch eine Rebellion in der südlichen Provinz Dhofar (Zufār) gefährdet, die sich Ende der 1960er Jahre mit südjemenitischer, sowjetischer und chinesischer Unterstützung in eine nationale Befreiungsbewegung unter dem zwischenzeitlichen Namen Popular Front for the Liberation of the Occupied Arabian Gulf (PFLOAG) transformierte. Mohammad Rezā hatte früh eine persönliche Beziehung zum omanischen Sultan Qābus ibn Sa'īd aufgebaut, der 1970 offenkundig mit geheimdienstlicher Unterstützung Großbritanniens - seinen Vater abgesetzt hatte. ${ }^{136}$ Der Schah offerierte dem Sultan während dessen Besuchs in Iran anlässlich der 2.500Jahr-Feier im Oktober 1971 Militärhilfe, ein Angebot, das Qābus wenige Monate später annahm. Iran behandelte den von marxistischen Kräften geführten Aufstand in Oman als Musterfall für die seit Langem beschworene radikale Penetration der Golfregion und entschloss sich daher zu einem direkten militärischen Eingreifen. ${ }^{137}$ Beginnend Ende 1972 kämpfte eine bis zu 4.000 Mann starke iranische Expeditionsarmee gegen die PFLOAG und leistete einen entscheidenden Beitrag zur letztendlichen Niederlage der Aufständischen im Jahr 1975. Der Schah nutzte die Intervention zur Erprobung seiner neuen Möglichkeiten zur Machtprojektion auf der arabischen Seite des Golfes - die C-130-Flugzeuge waren hier logistisch zentral -, nutzte den Konflikt mittels ständiger Rotation der entsandten Truppen zum Erwerb von Kampferfahrung für seine Streitkräfte, posierte als Verteidiger westlicher Interessen im Golf

\footnotetext{
$\overline{134}$ Vgl. COOPER, Oil Kings, S. 167f.; der Schah gewann allerdings die öffentliche Unterstützung der RCD-Partner wie auch Indiens für sein Prinzip, dass allein die Anrainerstaaten des Golfes für dessen Sicherheit Verantwortung tragen sollten. CHUBIN, Iran's Foreign Policy, S. 207ff.; zu den in wesentlichen Elementen entgegengesetzten USPlanungen zum Indischen Ozean in strategischer Hinsicht vgl. NSSM N 110 \{Follow-On Study of Strategy Toward the Indian Ocean \}, 22.12.70; , Report on NSSM 110 Follow-Up: Proposals for a US Naval Presence in the Indian Ocean', 9.6.71, encl. to Memo to Kissinger, 16.6.71, ,NSSM 110‘, NSSM Files, Box 12, RG 273, NACP; vgl. FAIN, Ascendance and Retreat, S. 194-7; Cābahār spielt bis zum heutigen Tage eine wichtige Rolle in den iranisch-indischen Beziehungen als potentielles Gegengewicht zu der angedachten militärischen Kooperation zwischen Pakistan und der Volksrepublik China bez. des (bis 1958 omanischen) Hafens Gwādar. Vgl. Tehran \#466, 22.1.72, DEF 15 IRAN; Eliot Jr. Memo, 14.12.72, DEF 6 IRAN; Tehran \#6649, 7.11.72, POL 15-1 IRAN, CF 70-73.

135 Den verhinderten Verkauf der U-Boote belegt auf Zugriff bislang nicht verfügbarer Quellen PALMER, Guardians, S. 91; zur Genese einer US-Strategie im Indischen Ozean vgl. SICK, Evolution.

136 Vgl. JIC(A)(70)6(Final), 17.4.70, CAB 186/5, TNA:PRO; überzeugend belegt jetzt in TAKRITI, Monsoon Revolution, S. 160-93.

${ }_{137}$ Jordanien leistete begrenzte Militärhilfe, doch mangelte es Amman an Ressourcen, um effektiv einzugreifen. Großbritannien behandelte Oman im Grunde als einen Satellitenstaat (,a special and potentially embarrassing relationship") und intervenierte bereits militärisch mit einem Aufgebot von etwa 1.000 Mann. Die britische Involvierung in Oman ist übrigens eine interessante Abkehr von der Rückzugspolitik infolge der East of SuezEntscheidung. Vgl. Douglas-Home Memo, DOP (70) 3, 29.6.70, CAB 148/101, TNA:PRO; vgl. TAKRITI, Monsoon Revolution, S. 284-308.
} 
und etablierte iranischen Einfluss nunmehr auf beiden Seiten der Straße von Hormus. ${ }^{138}$ Iranische Truppen blieben auch nach dem militärischen Sieg in Oman - ein weiteres Indiz für die breiteren strategischen Interessen des Schahs im Zuge der Intervention. Erst mit Ausbruch der Revolution 1978 endete die militärische Präsenz Irans bei dem Nachbarn.

Die Abkehr von einer allein auf den Golf selbst ausgerichteten maritimen Strategie eröffnete darüber hinaus die Möglichkeit einer extra-regionalen diplomatischen Initiative mit Zielsetzung neuer Allianzbildungen jenseits der von den Supermächten kontrollierten Blöcke. Wohl nicht zuletzt animiert durch die engen israelischen Verbindungen mit dem Apartheid-Regime in Südafrika baute auch Iran vertiefte Beziehungen zu Pretoria auf, gerichtet auf eine gemeinsame Kontrolle der westlichen Ausläufer des Indischen Ozeans entlang der afrikanischen Ostküste. ${ }^{139}$ Neben den Südafrikanern nannte der Schah in einem Gespräch mit dem Secretary of Defense James Schlesinger noch Australien und Singapur als mögliche militärische Partner in der Zukunft. ${ }^{140}$ Ein NIE der US-Geheimdienste vom Mai 1975 sprach explizit von dem langfristigen Ziel des Schahs, die militärische Präsenz sowohl der Sowjetunion wie auch der USA im Indischen Ozean vollständig zu beseitigen und eine regionale Dominanz zu erlangen. ${ }^{141}$ In diesem Kontext stellte der Schah ein weiteres Mal den Fortbestand von MIDEASTFOR in Bahrain in Frage. ${ }^{142}$ Jenseits des Indischen Ozeans engagierte sich der Schah nun auch global, z.B. im Kontext des Kalten Kriegs in Afrika. Notorisch wurde die nach der Revolution enthüllte Zusammenarbeit des kaiserlichen Iran im sogenannten Safari Club gemeinsam mit Frankreich, Saudi-Arabien, Ägypten und Marokko, eine Art privatem Geheimdienstclub, der in Abstimmung mit den Amerikanern gegen sowjetische bzw. radikale Kräfte in der Dritten Welt operierte und, unter anderem, zugunsten Mobutus in Zaire und Siad Barres im äthiopisch-somalischen Krieg intervenierte. ${ }^{143}$

\footnotetext{
${ }^{138}$ Die iranische Expeditionsarmee übte offenbar bald entscheidenden Einfluss auf die seitens Oman verfolgte Gesamtstrategie der Aufstandsbekämpfung aus. Die Iraner erlitten insgesamt 2.000 Verluste. Vgl. GoODE, Iranian Intervention, S. 451-4; das internationale Prestige Irans trug ebenfalls zum Sieg der omanischen Zentralgewalt bei - so beendete offenbar die Volksrepublik China ihre Unterstützung der PFLOAG auf Drängen des Schah. Vgl. Kissinger Memo, 6.9.74, FRUS 69-76, XXVII, S. 234ff.; vgl. auch MemCon, 23.7.73, ebd., S. 78-84; allerdings gab es auch Spannungen zwischen Omanern und Iranern über die militärische Gesamtstrategie. Vgl. CIA Staff Notes: Middle East, Africa, South Asia No. 0816/75, 26.6.75, CREST: \#RDP79*001-1; in den Zusammenhang iranischer Machtprojektion gehört auch das Drängen auf Lieferung von Tankflugzeugen. Vgl. Tehran \#5660, 6.10.71, ,Iran, Vol. IV, 1 Sep 71-Apr 73‘, NSCF, Country Files-Middle East, Box 602, RMNL.

${ }_{139}$ Außerhalb des Rahmens dieser Studie stehen die diversen Rüstungskooperationen zwischen Israel und Iran, gerade in Bereichen moderner Raketentechnologie und möglicher militärischer Nutzungen der Nuklearforschung. Auffallend sind die vielen Parallelen und Überschneidungen zur ähnlichen Vorgehensweise des südafrikanischen Minderheitsregimes. Vgl. auch CHEHABI, South Africa and Iran.

${ }^{140}$ Vgl. MemCon, 26.7.73, FRUS 69-76, XXVII, S. 113-118; später kamen noch Neuseeland und Indonesien als zukünftige Partner dazu.

${ }^{141}$ CIA, NIE 34-1-75 ,Iran‘, 9.5.75, ebd., S. 346-67, hier 363; vgl. bereits Kissinger Memo, 19.1.73, ebd., S. 19f.; vgl. auch BINDER, Regional Power; zur Belastung der iranisch-sowjetischen Beziehungen vgl. CIA Memo, 25.76, DNSA: IR01054.

${ }^{142}$ Vgl. Tehran \#5894, 11.7.74, ,Iran, Vol. VI, Jan74 -‘, NSCF, Country Files-Middle East, Box 603, RMNL; vgl. Tehran \#5694/\#4508, 11.7.74/14.5.75, FRUS 69-76, XXVII, S. 205f., 369ff.; Barr Memo, 14.1.76, ,Iran 1975, May-1979 Feb‘, Box 83, Haig Papers, LOC.

${ }^{143}$ Die Existenz des Safari-Clubs wurde nach der Revolution vom ägyptischen Journalisten Muhammad Heikal (Haykal) enthüllt, dem Einblick in die kaiserlichen Archive gewährt worden war. Vgl. HEIKAL, Return of the Ayatollah, S. 113f.; Haykal bewertet die Tätigkeiten des Clubs zurecht als „opera buffa“. Für eine andere Einschätzung vgl. MAMDANI, Good Muslim, Bad Muslim, S. 84-7; siehe auch DE MARENCHES/ADELMAN, Fourth
} 
Irans neues Interesse an Afrika und Asien sowie das Einschalten in die breitere Weltpolitik, insbesondere die zunehmende Kooperation mit der Volksrepublik China, illustrierten die globalen Ambitionen des Schahs und das Ziel der Rückgewinnung der einstmaligen Stellung des Perserreiches in der antiken Welt. Die Botschaft berichtete im Sommer 1974, infolge der jüngsten Steigerungen der Öleinnahmen in Kombination mit den geopolitischen Veränderungen ,,[...] Iran now enjoys greater ability to project its power and influence abroad than it has had for centuries."144 Man genieße in Teheran das wiedergewonnene Selbstbewusstsein als anerkannte Großmacht und insbesondere die demütigen Anfragen europäischer Ex-Weltmächte nach finanzieller Unterstützung. ${ }^{145}$ Diese unverhohlenen iranischen Ambitionen gerichtet auf eine regionale Vormacht- und eine globale Großmachtstellung blieben auch auf amerikanischer Seite nicht unbeachtet und stärkten diejenigen Stimmen, die bereits seit Längerem vor den Folgen einer übermäßigen Unterstützung des Schahs warnten. Allerdings wurden derlei Bedenken durch eine Reihe von strukturellen und personellen Gegebenheiten vorerst austariert. Trotz der Hinwendung der USPolitik zu einer gleichgewichtigeren Twin Pillars-Politik im Golf und der eigentlichen Ablehnung eines iranischen „Primats“ im Zuge der strategischen Debatten nach dem britischen Abzug ließ sich die demographische und militärische Überlegenheit Irans im Vergleich zu seinen Nachbarn nicht bestreiten. ${ }^{146}$ Zudem verzögerten die politischen Auswirkungen der zunehmenden Polarisierung in der Region durch den arabisch-israelischen Konflikt vorerst eine ernsthaftere Erwägung der bereits beschriebenen Avancen der Golfaraber an die Adresse der USA. Bei den meisten US-Offiziellen dominierte weiterhin die Wahrnehmung Irans als eine amerikanische Erfolgsgeschichte in der Dritten Welt. Angesichts des Traumas von Vietnam war die Infragestellung eines offenkundig pro-westlichen und stark antikommunistischen Verbündeten in einer strategisch zentralen Weltregion in der Tat kontraintuitiv. Von herausragender Bedeutung aber war die Tatsache, dass zu dieser Zeit viele überzeugte Pahlavists in den Führungspositionen der US-Administration zu finden waren, allen voran Sicherheitsberater Henry Kissinger, ab September 1973 zusätzlich Secretary of State, und Präsident Nixon selbst.

Zwar waren auch diese beiden nicht bereit, amerikanische Golfinteressen denjenigen Irans

World War, S. 158-61; offenkundig waren die USA treibende Kraft hinter Safari. Vgl. Kissinger Backchannel Message to Helms, 21.12.75, FRUS 69-76, XXVII, S. 448f.

${ }^{144}$ Tehran \#5268, 26.6.74, ebd., S. 195-203, hier S. 196; INR Intel Note RNAN-30, 29.6.73, POL IRANCHICOM, CF 70-73; vgl. Michaud Memo, ,Iran Reaches for Power', 24.6.74, DNSA: IR01137.

1451974 gewährte der Schah Milliardenkredite an Frankreich und Großbritannien. Vgl. FRUS 69-76, XXVII, S. 196 n2; CIA Intel Memo ,Iran: The Shah's Lending Binge‘, Dezember 1974, CREST: \#RDP85*014-8; vgl. auch Burrell/COTTRell, Iran, Afghanistan, Pakistan, S. 28ff., 34f.; vgl. auch CIA Study, ,Iran in the 1980s“, August 1977, DNSA: IR01210; CIA Memo, 5.10.77, DNSA: IR01229.

${ }^{146} \mathrm{Im}$ November 1974 hatte z.B. die US-Botschaft auf eine konsequentere Umsetzung der Gleichgewichtspolitik im Golf gedrängt, indem man Saudi-Arabien Zugang zu allen Waffensysteme gewähre, die man auch Iran zur Verfügung stelle, um wenigstens eine qualitative Balance zu wahren. Die Botschaft in Teheran erteilte solchen Bestrebungen sogleich eine Absage, da der Versuch des Aufbaus eine „military parity“ zwischen Saudi-Arabien und Iran im Grundsatz zum Scheitern verurteilt sei. Vgl. Tehran \#10090, 27.11.74, FRUS 69-76, XXVII, S. 272 f. 
unterzuordnen und sich einer vorwiegend militärischen Ordnungspolitik Teherans zu unterwerfen. Der persönliche Draht Mohammad Rezās aber wirkte sich dennoch günstig auf die Position dieser Offiziellen aus: Insbesondere Kissinger sprach sich in internen Debatten fast immer zugunsten der Berücksichtigung der Interessen des Schahs aus, häufig gegen die Bedenken seiner Berater und der militärischen Führung. ${ }^{147}$ Kissinger und Nixon empfanden eine Notwendigkeit, den Schah rückzuversichern, dass er weiterhin das „chosen instrument der USA in der Region sei, wenngleich die intern festgelegte Golfpolitik einer solchen Interpretation im Grunde widersprach. In jüngster Vergangenheit zugänglich gemachte Dokumente, aber auch die Tagebücher des engsten Schah-Vertrauten Asadollah 'Alam, belegen die Existenz eines ,geheimen Kanals“ zwischen Weißem Haus und Nijāwarān-Palast. Der USBotschafter in Iran, Richard Helms (1973-1976), war als ehemaliger CIA-Direktor der ideale „Conduit“ für diese geheime Korrespondenz, die sich offenbar nicht nur auf die Außenpolitik, sondern auch auf die amerikanische Innenpolitik erstreckte. ${ }^{148}$

Kissingers und Nixons Neigung zu ,persönlicher Diplomatie“ - im Übrigen geteilt von Mohammad Rezā - schien Iran einen Umweg hin zur gewünschten Anerkennung der eigenen regionalen Vormachtstellung durch die westliche Supermacht zu offerieren. Für Mohammad Rezā galt es, die eigene Position in den Jahren der fortbestehenden, wenngleich merklich reduzierten Abhängigkeit von den USA weiter auszubauen und dabei - vorerst - Konflikte mit Washington zu vermeiden. ${ }^{149}$ Der rigorose Antikommunismus der Administration ermögliche es Mohammad Rezā einige seiner regionalen Ziele in tatsächlicher Kooperation mit Washington zu verfolgen. An erster Stelle stand die weitere Schwächung des wichtigsten regionalen Widersachers: Irak. Wie bereits erwähnt, war die Hinwendung Iraks zu Moskau nicht zuletzt eine Reaktion auf die iranische Bedrohung seit Ende der 1960er Jahre gewesen. Trotz dieser recht augenfälligen Konsequenzen einer weiteren Konfrontationspolitik gegenüber Bagdad unterstützten Nixon und Kissinger während ihres Besuchs in Iran 1972 einen Vorschlag des Schahs, die bereits seit längerer Zeit durchgeführten verdeckten iranisch-israelischen Operationen zugunsten der aufständischen Kurden und Assyrer im Irak auch von amerikanischer Seite zu unterstützen. In den Folgejahren unterstützte die CIA die kurdischen Aufständischen finanziell wie auch durch Waffenlieferungen und hielt so die Baath-Regierung in Bagdad unter Druck. Nach dem irakischen Einlenken in Algiers im März 1975 hatten die Kurden um Bārzāni ihre Schuldigkeit getan: Im Stich gelassen von den USA und Iran, das nun die Grenze schloss und somit die Kurden ihrem Schicksal überließ, wurde der kurdische

\footnotetext{
${ }^{147}$ Siehe z.B. ebd., S. $270 n 7$.

${ }^{148}$ Henry Kissingers Nachlass in der Library of Congress ist bis auf Weiteres für die allgemeine Forschung nicht zugänglich - allerdings wurden die Dokumente für die Erstellung der FRUS-Bände eingesehen. Nixon hatte Helms zu einer Art Sonderbotschafter in Teheran berufen, ,[I]n charge of the area not only in charge of oil and so forth, but in terms of the stability of the governments, what we can do, frankly covertly and the rest [...]"; TelCon, 25.1.73, ebd., S. 23.

${ }_{149}$ Vgl. Walters Memo, 7.10.74, ebd., S. 247ff.; unbekümmert konstatierte der CIA-Mann: „I believe the U.S. can keep close to and benefit from this process and even influence Iran toward a positive regional and world role rather than a bid for area hegemony or other adventurism."; ebd., S. $248 f$.
} 
Aufstand von Saddam Husseins Regierung mit großen Verlusten an Menschenleben unterdrückt. Der ,sell-out“ der Kurden ging als besonders zynische Episode im Kalten Krieg in die Geschichte ein. Kissinger selbst kommentierte nur lapidar: „Covert action should not be confused with missionary work." 150

Die Unterstützung der Kurdenpolitik des Schahs mit dem Ziel der Schwächung Iraks und die Akzeptanz Washingtons der militärischen Interventionspolitik in Oman könnten, oberflächlich betrachtet, wieder als Indizien einer amerikanischen Unterstützung eines Primats Irans in der Region gewertet werden. ${ }^{151}$ Allerdings darf nicht übersehen werden, dass die Expedition in Oman auf Einladung des Sultans selbst stattfand, zu Beginn ohne jede Abstimmung mit Washington. Die Operationen gegen den Irak erfolgten zudem im Rahmen verdeckter Operationen. Eine unmittelbare militärische Ordnungspolitik Irans in der Golfregion - wie z.B. die Besetzung der Ölfelder auf der anderen Seite des Golfes - hätte aber wohl zum Konflikt mit den USA geführt, da Washington die Errichtung eines „Öl-Hegemons“ niemals hätte akzeptieren können. Dennoch kamen die Pahlavists in der Nixon-Administration dem Schah auch in der Frage iranischer Interessen auf der Arabischen Halbinsel für eine gewisse Zeit bedenklich weit entgegen. So war es in erster Linie der Unterstützung Nixons und Kissingers für den Schah zu verdanken, dass die sich seit NSSM N¹81 vom Mai 1973 intern abzeichnende Ausbalancierung der US-Politik gegenüber Iran auf der einen und Saudi-Arabien auf der anderen Seite nicht stärker auswirkte. Nixon und andere Pahlavists befürworteten die Zuweisung einer (informellen) Rolle für Iran im Falle interner Unruhen in Saudi-Arabien. In Gesprächen mit dem iranischen Monarchen während dessen USA-Besuchs im Juli 1973 vereinbarten Kissinger und Nixon in einer Geheimabsprache mit dem Schah gemeinsame Ernstfallplanungen für ein militärisches Eingreifen Irans im Falle eines Umsturzes. „We have no intention of accepting a Qadhafi in Saudi Arabia“, so Kissinger in seiner Begründung für

${ }^{150} \mathrm{Vgl}$. MemCon, 29.5.69; Helms Memo, 31.3.72; Saunders Memo, 7.6.72; Kissinger Memo, o.D. [Juli-August 1972]; Kissinger Memo, 5.10.72, FRUS 69-76, E-4, docs. 258, 303, 313, 322, 325; vgl. auch ebd., doc. $200 n 1$; Kissinger Memo, 29.3.73; Colby Memo, 7.8.73; Kissinger Memo, 6.9.73; Kissinger Memo, 11.4.74; Kissinger Memo, 24.6.74; MemCon, 26.8.74, FRUS 69-76, XXVII, S. 605f., 646ff., 662ff., 686f., 701, 707f.; die Iraker beantworteten die iranische Unterstützung der Kurden mit eigener verdeckter Unterstützung für aufständische Belutschen im iranischen Südosten. Vgl. ed. note in ebd., S. 600; TelCon, 29.3.74, DNSA:HAKTelCons: \#KA12243; Saunders Memo, 7.6.72, DDRS: CK3100668720; Summary of Meetings, 31.10.74, DDRS: CK3100646354; vgl. auch BILL, Eagle and Lion, S. 205-8; LiTTLE, United States and the Kurds, S. 74-85, Kissinger-Zitat auf S. 83; GIBSON, Sold Out?, S. 143-97.

${ }^{151}$ Neben Oman und Irak unterstützten die USA auch gemeinsame saudisch-iranische Operationen gegen den kommunistischen Südjemen. Vgl. MemCon, 24.7.73, FRUS 69-76, XXVII, S. 88-94; der Schah versuchte zudem - mit Einverständnis der USA - Afghanistan nach dem Sturz der Monarchie 1973 mittels massiver Wirtschaftshilfe von einem einseitig pro-sowjetischen Kurs abzuhalten. Vgl. Kissinger Memo, 6.9.74, ebd., S. 234ff.; die USA unterstützten zudem die iranischen Beistandsgarantien an Kuwait im Kontext des von Iraks provozierten Grenzzwischenfalls 1973. Der Schah warnte in mehreren Interviews, man werde eine irakische Besetzung Kuwaits nicht hinnehmen. Vgl. Baghdad \#191, 9.4.73, ebd., S. 614f.; BuRRELL/COTTRELL, Iran, Afghanistan, Pakistan, S. 6f.; vgl. auch Oakley Memo, 2.1.75, DDRS: CK3100671366; im Oktober 1972 durfte sich der Schah als wichtiger globaler Verbündeter der USA fühlen, als er im Rahmen der Operation Enhance Plus von Washington gebeten wurde, sämtliche 90 F-5A seiner Luftwaffe an die Republik Südvietnam im Kontext der Aufrüstung Saigons als Teil der sich abzeichnenden Friedensregelung in Indochina zu übergeben. Der Schah gab schließlich 36 Maschinen frei, auch Südkorea und Taiwan überstellten Teile ihrer Luftwaffe. Im Gegenzug sagte man Iran eine schnellere Lieferung der bereits georderten $F-5 E$ - und $F$-4-Kampfflugzeuge, einen temporären lease einer weiteren $F$-4Staffel, sowie eine bessere Bewaffnung für diese zukünftigen Systeme zu. Vgl. State \#192358, 20.10.72; Saunders Memo, 1.12.72, FRUS 69-76, E-4, docs. 224, 237. 
dieses eigenmächtige Vorgehen. ${ }^{152}$

Die von Kissinger initiierte Geheimhaltung dieser Absprachen mit den Iranern auch vor großen Teilen der eigenen Sicherheitsbürokratie und insbesondere vor der eigenen militärischen Führung hatte allerdings zur Folge, dass diese Planungen in der Folgezeit eher virtuellen Charakter hatten. $^{153}$ In gewisser Weise standen diese Absprachen im Gegensatz zu der formalen Ausdifferenzierung der amerikanischen Grand Strategy mit Bezug auf die Golfregion in dieser Zeit. Da ohne Beteiligung der Bürokratie erfolgt, ist die tatsächliche Bedeutung dieser Zusagen nur schwer abzuschätzen. Als es im Kontext des Vierten Arabisch-Israelischen Krieges im Oktober 1973 und des arabischen Ölembargos zu Andeutungen einer Wende in der US-Sicherheitsstrategie im Persischen Golf kam, zeigte sich ein weiteres Mal, dass amerikanische und iranische strategische Vorstellungen nicht deckungsgleich waren. Secretary of Defense James Schlesinger drängte zwischenzeitlich sogar auf eine militärische Besetzung der Ölfelder als Reaktion auf das Embargo und Kissinger warnte in einer Pressekonferenz im November vor möglichen militärischen Konsequenzen. ${ }^{154}$ Die Nixon-Administration entsandte eine Task Force um den Flugzeugträger Hancock an die Küste Omans, offenbar als Drohgebärde gegenüber den Golfarabern intendiert. Iran unterstützte die Flottenpräsenz, indem es die Task Force mit Treibstoff versorgte und zudem (geheim gehaltene) Aufklärungs- und Versorgungsflüge von iranischen Flugfeldern in Bandar 'Abbās genehmigte. Zwar zog die Task Force bald wieder ab - die erschrockenen Saudis zeigten sich rasch kompromissbereit -, doch wurden die amerikanischen Aufklärungsflüge auch im Anschluss weiter fortgesetzt. ${ }^{155}$

152 Deputy Director for Central Intelligence Vernon Walters warnte Kissinger explizit: „Iranian military intervention in the Peninsula would arouse the Arab world."; Senior Review Group Meeting, 20.7.73, FRUS 6976, XXVII, S. 77; Clements vom DOD warnte vor der Absicht des Schahs, jede Gelegenheit zur militärischen Intervention in den VAE wahrzunehmen. Für die Reaktion der Pahlavists wie Kissinger und Helms auf solche Warnungen siehe MemCons, 23./24.7.73, ebd., S. 78-84, 95-109, hier 99-103; Kissingers Bemerkung auf S. 100; offenbar ging die Idee einer Absprache im Falle von Instabilität in Saudi-Arabien auf eine Anregung Helms“ zurück. Vgl. Saunders Memo, 23.7.73, ,Iran, Vol. V, May 73-Dec 73‘, NSCF, Country Files-Middle East, Box 603, RMNL; CIA \#WH31813, 12.7.73, CIA-Helms.

${ }_{153}$ Vgl. MemCon, 24.7.73, FRUS 69-76, XXVII, S. 95-109, hier 102; Kissinger bestand darauf, dass sämtliche Absprachen nur persönlich mit Botschafter Helms getroffen und keine schriftlichen Zusagen gegeben werden sollten. Auch der Schah bekräftigte die Notwendigkeit, alle Planungen bezüglich Saudi-Arabien ,hush-hush“ durchzuführen. Ebd., S. 103; im Oktober 1973 berichtete Helms per Backchannel-Nachricht über entsprechende Konsultationen mit dem Schah. Siehe ebd., S. 75 n7; den neuen Präsidenten Ford informierte Kissinger später lapidar: „No one knows that Nixon promised him support for an Iranian operation against a new regime."; MemCon, 5./15./15.5.75, ebd., S. 343, 371f., 372-9; vgl. COOPER, Oil Kings, S. 96-106; der Schah nutzte den „Helms"-Kanal auch immer wieder, um direkte Nachrichten an Nixon zu senden und dabei das State Department zu umgehen. Siehe z.B. CIA \#sanitized to Kissinger, 6.4.73, CIA-Helms; Helms wiederum zeigte sich besorgt, der Schah „may be overreading this exercise“ und befürchtete ,some ill-advised move or inquiry which might get back to the Saudis.“; später konstatierte Helms: „I am sure my client is willing, but I wish HAK \{Kissinger; R.P.\} had given the job to some Iranian on a slightly lower level."; CIA \#sanitized to Scowcroft, 4.9.73; vgl. CIA \#WH31980, 9.9.73; CIA \#sanitized to Scowcroft/Saunders, 15.9.73; Study Paper, attached to Helms Letter, 17.9.73; CIA \#100, 11.10.73; CIA-Helms.

154 Vgl. ed. note, FRUS 69-76, XXXVI, S. 690-3; für den sich andeutenden Strategiewandel vgl. MemCon, 29.11.73, ebd., S. $711 \mathrm{ff}$.

${ }_{155}$ Die Aufklärungsflüge mit Lockheed P-3-Flugzeugen hatten offenkundig die Lage auf der arabischen Seite des Golfes zum Ziel. Vgl. State \#217485, 4.11.73; Tehran \#8321, 26.11.73; Ellsworth Memo, 8.1.75, FRUS 69-76, XXVII, S. 153f., 159f., 291f.; intern gab es durchaus Bedenken gegen die Fortsetzung der Flüge: „Bandar Abbas should not take shape as US base in Iran."; Tehran \#5088, 2.6.75, ebd., S. 400; vgl. auch ed. note, FRUS 69-76, XXXVI, S. 690-3; ed. note, FRUS 69-76, XXXVII, S. 108ff.; vgl. SecState \#81647, 10.4.75, DDRS: CK3100593610. 
Wenngleich die Episode als Beispiel für die Kooperation zwischen beiden Mächten in Sicherheitsfragen des Golfs gewertet werden kann, so ergaben sich doch aus dem Gesamtzusammenhang strategische Schlussfolgerungen, die eine Fortsetzung der bisherigen Politik eher in Frage stellten und Mitte der 1970er Jahre zu scharfen internen Auseinandersetzungen innerhalb der US-Administration führen sollten.

Durch das Ölembargo war man auf die möglichen schwerwiegenden Folgen der Abhängigkeit der westlichen Welt vom nahöstlichen Erdöl in vollem Ausmaß aufmerksam geworden. Politische und ökonomische Drohungen hatten die Golfaraber nicht zum Einlenken gebracht erst die öffentlichen Spekulationen über ein militärisches Eingreifen im Golf hatten saudisches Entgegenkommen induziert. Angesichts dieser Entwicklungen musste Paul Erdmans Szenario eines hegemonialen „Ölkriegs“ und einer iranischen Kontrolle der Golfregion, beunruhigend wirken, zumal der Schah die zuvor gemäßigte iranische Ölpreispolitik inzwischen revidiert hatte. Für US-Planer war die Vorstellung eines zukünftigen Embargos unter Beteiligung Irans - auf dem Papier nunmehr eine militärische Großmacht - ein Horrorszenario. In Kombination mit einer denkbaren Nuklearisierung Irans infolge des geplanten gewaltigen zivilen Nuklearprogramms drohte de facto ein strategischer Ausschluss aus der weltpolitisch zentralen Golfregion. Eine Einschätzung der DIA vom April 1973 warnte vor einer möglichen Abenteuerpolitik des Schahs im Anschluss an die Vollendung seiner Aufrüstungspolitik um das Jahr 1980. Zeitungsberichte in den USA zitierten ein angebliches CIA-Profil des Schahs, in welchem jener als ,[...] dangerous megalomaniac, who is likely to pursue his own aims in disregard of U.S. interests" beschrieben wurde. ${ }^{156}$ Ein internes Papier warnte Secretary Schlesinger angesichts der Gefahr, die USA könnten in „militärische Abenteuer“ Irans hineingezogen werden. Der Schah, so die JCS, sei nun „extremely protective“ bezüglich der Straße von Hormus - und warnten ein weiteres Mal vor den langfristigen Ambitionen Mohammad Rezās. ${ }^{157}$ Ein anderes internes DOD-Papier stellte fest, die Wahrnehmung des bilateralen Verhältnisses als ,,mature relationship“ seitens des State Department sei letztendlich haltlos. Es gebe eine ganze Reihe absehbarer und gravierender Streitpunkte zwischen beiden Ländern, insbesondere den Ausschluss amerikanischer Macht aus dem Golf, doch gebe es keine Versuche, diese Fragen auch nur im Ansatz mit Iran zu diskutieren. ${ }^{158}$ Ein NIE zu Iran vom Mai 1975, den Konsens der amerikanischen Intelligence Community wiedergebend, reflektierte schließlich die auseinanderdriftenden strategischen Interessen Irans und der USA im Persischen Golf und im Indischen Ozean:

US-Iranian relations are likely to become more difficult in coming years. The Shah is seeking to remove both the US and Soviet military presence from the Persian Gulf and the Indian Ocean and to establish his own regional collective security arrangements in the Gulf; while he will

\footnotetext{
${ }^{156}$ Zit. aus PALMER, Guardians, S. 91; die CIA fertigte in der Tat psychologische Profile weltpolitisch bedeutsamer Persönlichkeiten an. Die Studie selbst ist allerdings nicht deklassifiziert.

157 JCS History Iran, S. 113f.

158 Siehe FRUS 69-76, XXVII, S. 380 n2.
} 
tacitly approve of US naval operations in these waters as long as the USSR keeps naval vessels on station there, we cannot depend on him to provide access to Iranian facilities to support fleet units, and he will probably use his influence to end our use of Bahrein. ${ }^{159}$

Das etablierte Bild des iranischen Monarchen als aufgeklärter Herrscher und enger Freund des Westens in der amerikanischen Öffentlichkeit erlitt in dieser Zeit Risse, in erster Linie eine Folge der Rolle, die der Schah bei den sprunghaften Ölpreiserhöhungen gespielt hatte. ${ }^{160}$ Finanzminister William E. Simon bezeichnete den Schah intern im Sommer 1974 als den eigentlichen „Ringleader“ hinter den exorbitanten Ölpreissteigerungen und empfahl eine Umkehr der Iranpolitik, unter anderem ein Waffenembargo gegen Iran, um Teheran zum Einlenken zu zwingen, und darüber hinaus eine verstärkte Hinwendung zum schutzbedürftigen und eher willfährigen Saudi-Arabien. Wenig überraschend sprach sich Kissinger vehement gegen einen solchen Politikwandel aus. ${ }^{161}$ Dennoch gelangte Simons harsche Kritik an der Politik des Schahs an die Öffentlichkeit. Ein US-Magazin zitierte den Finanzminister mit den Worten: „The Shah is a nut.“ Auch in Teheran merkte man nun, dass sich die Zeiten fast einstimmiger Unterstützung seitens der amerikanischen Administration dem Ende zuneigten. ${ }^{162}$

Im September 1975 stellte schließlich Secretary of Defense James Schlesinger in einem Memorandum an Präsident Ford fest, dass es ,some doubt as to whether our current policy of supporting an apparently open-ended Iranian military buildup will continue to serve our longterm interests" gebe. Schlesinger regte angesichts der Schwierigkeiten Irans, unzählige hochmoderne Rüstungsgüter zu absorbieren und der zudem daraus resultierenden rasant ansteigenden Zahl von Amerikanern in Iran in Ausbildungs- und Unterstützungsfunktionen, eine kritische Überprüfung der „Blankoscheck“-Politik an. Nur am Rande erwähnte Schlesinger die Aussicht auf zukünftige Belastungen des bilateralen Verhältnisses, die aber offenkundig ein zentraler Beweggrund für seinen Initiative war:

Despite the basic foundation of good relationships, there are prospects that US-Iranian relations will become more difficult in the years ahead for a variety of reasons $[\ldots]$-pressures for various

\footnotetext{
${ }^{159}$ NIE 34-1-75 \{Iran\}, 9.5.75, ebd., S. 346-67, hier S. 348.

160 Zur Welle von Schah-kritischen Artikeln ab Oktober 1974 vgl. ed. note, ebd., S. 253ff.; auch der bereits erwähnte Erdman-Artikel gehörte dazu. Wie üblich reagierte die iranische Regierung auf diese Veröffentlichungen mit großer Empörung. Der Kolumnist Jack Anderson machte die im Zuge der Watergate-Untersuchungen kolportierten Wahlkampfspenden an Nixon durch den Schah publik. Vgl. oben S. 462, Anm. 274.

${ }_{161}$ Vgl. MemCons, 9./30.7./3.8.74, FRUS 69-76, XXXVI, S. $1022 \mathrm{ff} ., 1025 \mathrm{ff} ., 1027 \mathrm{ff} . ;$ vgl. ed. note, FRUS 69-76, XXVII, S. 207 ff.; S. 215 n2; ebd., S. 270 n7; offenbar hatte Simon die Saudis gewarnt, Iran habe nur noch 15 Jahre Ölförderung zu erwarten, während die saudische Produktion noch 150 Jahre laufen würde. Somit habe der Schah die Absicht, nach Versiegen der eigenen Produktion die saudische zu übernehmen - offenbar meinte Simon damit eine gewaltsame Übernahme. Verteidigungsminister Schlesinger beschwerte sich gegenüber Kissinger über Simons ,verrückter Idee“ „of breaking the Shah“. Vgl. auch MemCons, 13./17.8.74, FRUS 69-76, XXXVII, S. 111, 12ff.; MemCon, 2.8.74, ISCAP.

${ }_{162}$ Helms in Washington beschwerte sich bitterlich über die öffentliche Kritik am iranischen Monarchen. Vgl. Helms Backchannel \#936, 18.7.74, FRUS 69-76, XXVII, S. 212ff.; vgl. bereits früher in State \#150380/\#152053, 11./14.7.74; Tehran \#5716, 12.7.74, CFPF, 1973-79/Electronic Telegrams, RG 59, NACP; Nixon hatte sich bereits einige Monate zuvor für einige öffentliche Bemerkungen Simons bez. Iran bei Mohammad Rezā entschuldigt. Vgl. Nixon Letter, 23.3.74; Saunders Memo, 3.4.74, ,Iran, Corres. M.R. Pahlavi, Shahanshah of Iran', NSCF, Presidential Correspondence, Box 755, RMNL; siehe auch TelCons, 15.7.74, 2.6.76, DNSA:HAKTelCons: \#KA12493 und \#KA14938; vgl. auch COOPER, Showdown.
} 
arms the US may not wish to release, the Shah's interest in the removal of both the US and Soviet military presence from the Persian Gulf and Indian Ocean areas, Gulf Arab doubts concerning Tehran's ultimate intentions plus possible overt rivalries which would pose difficult choices for the US, and the Shah's role in OPEC, among others. The issue of oil prices obviously is one in which US interests and the Shah's perceptions of his interests could easily collide, and soon. The question of nuclear safeguards for the reactors he seeks is also likely to be troublesome. ${ }^{163}$

Der Widerstand in der Administration gegen einen so grundsätzlichen Kurswechsel in der amerikanischen Iranpolitik aber war groß. Zu etabliert war das enge bilaterale Verhältnis, zu engmaschig die vielen Netzwerke zwischen den iranischen Offiziellen in Washington und Teheran, den verschiedenen Pahlavists, und den höchsten Stellen in der Verwaltung, als das eine Revision der einmütigen Unterstützung Mohammad Rezās ernsthaft in Betracht gezogen werden konnte. Schlesingers Initiative regte aber immerhin eine neue Studie zur amerikanischen Grand Strategy im Persischen Golf an. ${ }^{164}$

Hauptgegenstand der inneren Debatten war der Mehrwert der engen sicherheitspolitischen Anlehnung an den Iran Mohammad Rezās. Die Befürworter einer special relationship mit Teheran führten die vielen regionalpolitischen Vorteile ins Feld - Irans (wenn auch verdeckte) Unterstützung Israels, die antikommunistische Ausrichtung und die Gegnerschaft Irans gegen anti-westlichen radikalen arabischen Nationalismus und die Möglichkeit, Operationen „auszulagern“, wie z.B. im Rahmen des Safari Club. Selbstverständlich durfte auch ein Verweis auf die Duldung der US-Abhör- und Messstationen nicht fehlen. Die enge Rüstungskooperation - so das Argument - garantiere dabei das Fortdauern der engen Bindungen und einen grundsätzlichen Einfluss auf die Zielsetzungen iranischer Politik. Eine Vernunftpartnerschaft habe somit das alte Klientelverhältnis ersetzt, mit großen Vorteilen für beide Seiten. Die US-Botschaft pries diesen ,new texture which has been introduced into our relations“, und identifizierte Nixons Teheran-Besuch vom Mai 1972 als entscheidenden

163 Schlesinger Memo, 2.9.75, FRUS 69-76, XXVII, S. 424, 429; Schlesinger monierte die enormen Waffenbestellungen des Schahs und die mangelnde Fähigkeit der iranischen Streitkräfte, die neuen Systeme zu absorbieren. Zudem hätten sich die Streitkräfte Irans seit 1967 mit dem Wachstum auf $436.000 \mathrm{Mann}$ mehr als verdoppelt und eine weitere Expansion sei im Gange. Die Verteidigungsausgaben seien von 6,8\% des Bruttosozialprodukts auf $15,2 \%$ in 1975 gestiegen. ISA im DOD wandte sich zeitgleich ebenfalls gegen die Vertreter der „don't rock the boat"-These innerhalb der Administration, für die das Verhältnis zum Schah sakrosankt war. Das Papier wurde zurückgehalten. Siehe ebd., S. $429 n 4$; Kissinger sprach gegenüber Ford von einer „vicious campaign“ von Treasury und DOD gegen Iran. Vgl. CoOPER, Showdown, S. 581; Coopers Interpretationen sind allerdings von einem wenig angebrachten verschwörungstheoretischen Stil geprägt und zeugen von einer eher begrenzten Kenntnis der strategischen Realitäten. Vgl. Sisco Memo, 9.5.75, DDRS: CK3100001896; generell zu Schlesinger und Iran vgl. SICK, All Fall Down, S. 15-8.

${ }^{164}$ Vgl. Granger/Oakley Memo, 23.9.75, FRUS 69-76, XXVII, S. 434ff.; zur neuen Golfstudie vgl. ed. note, ebd., S. 518f.; zur Direktive siehe ,U.S. Policy Toward the Persian Gulf ' [NSSM N²38], 13.2.76, , NSSM 238‘, NSSM Files, Box 16, RG 273, NACP; vgl. Middle East Study Group, National War College, Committee Report, Mai 1975, DDRS: CK3100046738; MemCon, 2.9.75, DDRS: CK3100649584; Tehran \#10801, 5.11.75, DDRS: CK3100583140; die neue Golfstrategie wurde erst Anfang 1977 fertiggestellt und liegt außerhalb des Rahmens der vorliegenden Studie. Für die längerfristige Entwicklung der US-Golfpolitik im Anschluss vgl. SICK, United States in the Persian Gulf; für eine zeitgenössische Einschätzung, die Deckungsgleichheit der beiderseitigen Interessen unterstellte, vgl. RAMAZANI, Enduring Friendship; der später geschriebenen JCS History zu den bilateralen Beziehungen zufolge gab es im Februar 1976 eine Weisung des Deputy Secretary of Defense, die im Kern den Automatismus der „Blankoscheck“-Politik aufhob. Allerdings bekräftigt die Studie selbst, dass damit keine Kehrtwende in der bisherigen Politik intendiert gewesen wäre. Vgl. JCS History Iran, S. 116f. 
Wendepunkt. ${ }^{165}$ Daher seien auch die wenig subtilen Versuche der Gegner dieser Politik, die „Blankoscheck“-Politik durch administrative Verzögerungstaktiken in Frage zu stellen, letztlich kontraproduktiv und schadeten der Glaubwürdigkeit der amerikanischen Politik in der Region. ${ }^{166}$

An die Öffentlichkeit getragen wurde diese Debatte über den „Blankoscheck“ infolge der Anhörungen im US-Senat über die umfangreichen Waffenexporte nach Iran. In einem gewandelten innenpolitischen Klima in den USA nach Vietnam-Debakel, Watergate und CIAEnthüllungen gab es ein großes öffentliches Interesse an der eigenen Politik in der sogenannten Dritten Welt, und gerade an absehbaren außenpolitischen Fehlentwicklungen. Die Zusammenarbeit mit dem autoritären Schah und das etablierte Entgegenkommen hinsichtlich der exorbitanten Aufrüstungswünsche Irans mussten zwangsläufig in dieser Atmosphäre viel Aufmerksamkeit erfahren. ${ }^{167}$ Ein Subcommittee des Senate Committee on Foreign Relations unter dem Vorsitz des ehemaligen Vizepräsidenten Hubert Humphrey veröffentliche im August 1976 eine äußerst kritische Studie über den Anstieg der amerikanischen Waffenverkäufe an Iran. Im Kern war die Studie eine Abrechnung mit der „Blankoscheck“-Politik Nixons und Kissingers, dabei aber zugleich die bereits dargelegte Vorgeschichte und Pfadabhängigkeit der Entscheidung vom Mai 1972 vertuschend. Die hauptsächliche Kritik der Studie richtete sich gegen die rasante Ausweitung der Waffenexporte an Iran, welches kaum in der Lage sei, das eingehende Material zu absorbieren bzw. auch nur zu unterhalten. Daraus resultiere die Präsenz einer immer weiter steigenden Zahl von Ausländern und insbesondere US-Amerikanern in Ausbildungs- und Wartungsrollen mit unvorhersehbaren langfristigen Konsequenzen für das bilaterale Verhältnis. Bemerkenswert waren insbesondere die Feststellungen zu den Folgen der fast schon symbiotischen Verbindung zwischen den USA und Iran im militärischen Feld. Die Vereinigten Staaten könnten ihre laufenden Rüstungsexporte an Iran nur unter Inkaufnahme einer „major crisis“ in den Beziehungen einstellen - allerdings sei gleichzeitig der Handlungsspielraum Mohammad Rezās stark eingeschränkt. Ein Bruch mit den USA hätte eine gewaltige Schwächung der iranischen Verteidigungskraft zur Folge aufgrund der technologischen Pfadabhängigkeiten, die sich aus den Jahrzehnten der engen sicherheitspolitischen Zusammenarbeit ergäben. ${ }^{168}$ Theoretisch, so die Studie, hätten die

\footnotetext{
165 „This comes about because we have mastered $\{$ the; R.P. $\}$ tendency in recent years to second-guess $\{$ the $\}$ Shah about his policies while Iran in turn has recovered from its client status attitude."; Tehran \#4639, 2.7.73, FRUS 69-76, XXVII, S. 63f.; Helms Telegramm ist eine einzige Lobpreisung des Schahs. Vgl. Kissinger Memo, 6.9.74, ebd., S. 234ff.; Helms nutzte die Stationen bereits als DCI als Faustpfand für die weitere Unterstützung des Schahs. Siehe Helms Memo., 16.4.70, FRUS 69-76, E-4, doc. 63.

${ }_{166} \mathrm{Im}$ Herbst 1974 beschwerte sich Helms über das wachsende Misstrauen der Iraner angesichts des dilatorischen Behandelns ihrer Rüstungsanfragen an das DOD, insbesondere was iranische Wünsche bezüglich Koproduktion technologisch fortgeschrittener Waffensysteme anging, wie z.B. Maverick-Luft-Boden-Raketen, StingerLuftabwehrraketen und TOW-Panzerabwehr-Lenkwaffen. Vgl. Helms Backchannel \#974, 4.9.74, FRUS 69-76, XXVII, S. $231 \mathrm{ff}$.; Eagleburger Memo, 16.9.74, DNSA: IR00891.

${ }_{167}$ Zur veränderten Atmosphäre im Washington Mitte der 1970er Jahre vgl. SCHMITZ/WALKER, Foreign Policy of Human Rights.

168 Senate Subcommittee, Sales to Iran, S. Xf.; ähnlich die Botschaft bereits im Vorjahr. Vgl. Tehran \#A-19, 28.1.75, Asnād 7, S. 130-44; für die Salienz dieser Argumentation vgl. NoYES, Clouded Lens, S. $123 \mathrm{f}$.
} 
Befürworter einer engen militärischen Kooperation recht mit ihrer Behauptung, aus der Abhängigkeit ergäbe sich automatisch auch Einfluss auf die Politik des Partnerlandes: „In theory, the U.S. has the capability to immobilize major components of the Iranian armed forces, especially the Air Force, by cutting off spares, munitions and maintenance support should Iran try to use U.S. equipment for purposes contrary to important U.S. interests." Dieses Drohpotenzial aber sei eher virtuell, da ein solches Embargo zwangsläufig einen Zusammenbruch der Beziehungen zur Folge haben würde - selbst ein Szenario, in welchem die amerikanischen Berater zu „Geiseln“ in Iran werden könnten, sei nicht auszuschließen, so die Studie fast prophetisch warnend. ${ }^{169}$

Henry Kissinger bezeichnete den Bericht intern als „Desaster“ - im Kern aber bewirkte auch die öffentliche Debatte im Zuge der Veröffentlichung der Senats-Studie keine Umkehr in der Waffenexportpolitik gegenüber Iran. ${ }^{170}$ In den Jahren der Nixon- und Ford-Administration setzte sich die Aufrüstungspolitik Irans mit williger Unterstützung der amerikanischen Supermacht im Grunde ungebrochen fort. Im Kern stand weiterhin der Ausbau der iranischen Luftwaffe. Nach der Entscheidung zugunsten von 80 F-14 Tomcat-Kampfflugzeugen im Juni 1974 im Wert von \$1,8 Milliarden anstelle einer Kombination von F-14- und F-15-Jets war die generelle Richtung bereits vorgegeben. Jedes amerikanische Hochtechnologiesystem, das sich gerade im Entwicklungsstadium befand, konnte sich der Aufmerksamkeit Mohammad Rezās sicher sein. Bereits im Mai 1975 informierte der Schah die US-Administration über seine Absichten, seine noch kaum gealterten $F$-4- und F-5-Staffeln zum Ende der Dekade mit brandneuen $F$-16- und sich erst in der Entwicklung befindenden $F$-18-Kampfflugzeugen zu ersetzen. Immer exzentrischer wurden dabei die Stückzahlen: 280-300 Maschinen vom Typ $F$ 16 sah der Schah als notwendig an, sowie 240-250 Mehrzweckkampfflugzeuge vom Typ $F-18$ (später $F / A$-18) für seine Marine. ${ }^{171}$ So gewaltig war der absehbare Umfang der iranischen Streitkräfte in der Zukunft, dass selbst Moskau sich nun bemüßigt sah, die unaufhörliche

\footnotetext{
169 Zit. aus SenAte SubCOMmitTEE, Sales to Iran, S. X; S. 52: „More specifically, if there were a revolution in Iran and the Shah were replaced by an anti-U.S. regime, that regime would find it virtually impossible to maintain the current inventory of U.S. weapons without sustained cooperation with the United States. This might moderate a new regime's policies. However, if the regime were intent upon eliminating the U.S. role and presence in Iran, the United States could retaliate by bringing Iran's military machine to a virtual standstill."; vgl. EMERY, US and Revolution, S. 155-8; die hohen Menschenverluste Irans im Krieg mit Irak in den 1980ern sind nicht zuletzt der Immobilisierung der modernen Waffensysteme durch ausbleibende US-Unterstützung zuzuschreiben. Die Feststellung des ehemaligen Botschafter Helms, ,[...] in 1980, when Saddam Hussein's army finally did march into Iran, it was this expensive equipment that kept Iran and the mullahs from losing that long, bitter war", kann nur als obszön bezeichnet werden; HeLMS, Look over my Shoulder, S. 419; für ein Beispiel wie die Ersatzteilfrage nach der Revolution politisch instrumentalisiert wurde, vgl. State \#262958/21, 6.10.79, DNSA: IR03229.

${ }^{170}$ Siehe FRUS 69-76, XXVII, S. 535 n4; Kissinger machte anti-iranische Kräfte in der Administration und die Pro-Israel-Lobby für den Druck gegen Waffenexporte verantwortlich. Vgl. ebd., S. 541 n7; die Administration erwog eine öffentliche Gegendarstellung zum Senatsbericht, sah aber davon ab. Siehe den sehr ,pahlawistischen“" Entwurf in Oakley Memo, 6.8.76, ebd., S. 540-3; zum Humphrey-Bericht vgl. auch BILL, Eagle and Lion, S. $211 \mathrm{ff}$. ${ }^{171} \mathrm{Im}$ Sommer 1976 erfolgte schließlich die erste Order über 150 Maschinen vom Typ $F-16$ mit Option auf 150 weitere. Nur wenige Monate später orderte der Schah 250 Maschinen vom Typ F-18L; Vgl. MemCon, 24.7.73, POL 7 IRAN, CF 70-73; vgl. ed. note; Tehran \#4744, 11.6.74; MemCon, 16.5.75; Atherton Memo, 29.7.76; Scowcroft Memo, 27.10.76, FRUS 69-76, XXVII, S. 32f., 193ff., 380-3; 533-6, 566-9; hinzu kamen Pläne für mehrere Geschwader an Transport- und Kampfhubschraubern. Vgl. Report ,Iranian Helicopter Program Options', 12.12.74, encl. to Hallock Memo, 21.12.74, ,Defense Dept., Hallock, Richard, Document Set \#1 1974, Dec', Box 52, Schlesinger Papers, LOC; Information Nr. 409/78, 18.7.78, BStU, MfS, HV A, Nr. 71.
} 
Aufrüstung des Schahs gegenüber den Amerikanern anzusprechen: Iran habe keine geopolitischen Zwänge für seine monströsen Verteidigungsanstrengungen. Somit stelle sich die Frage, welchem letztendlichen Zweck diese Aufrüstung diene. ${ }^{172}$ Wie bereits geschildert, waren in der Ford-Administration durchaus ähnliche Stimmen zu vernehmen. Wenngleich ein iranisches Primat in der Golfregion sowohl im Kontext von NSDM N92 vom November 1970 sowie in den Studien in der Nachfolge von NSSM N 181 vom Mai 1973 als Option abgelehnt worden war, unterlief die Waffenexportpolitik im Grunde die eigenen strategischen Zielsetzungen. Nur halbherzig ging die von Schlesinger angestoßene Überprüfung der USGolfpolitik die Frage der Rüstungsexporte an. ${ }^{173}$ Ähnlich wie im östlichen Mittelmeer bewirkte die US-Politik auch in der Großregion des Persischen Golfes gewaltige militärische Ungleichgewichte, die mittelfristig zur Destabilisierung der Subregionen beitragen sollten. ${ }^{174}$

Selbst die Carter-Administration mit ihrer ostentativen Menschenrechtspolitik sollte im Falle Irans eine Ausnahme machen - zu etabliert war das enge Verhältnis, zu groß inzwischen der Einfluss der Pahlavists in Regierungsbürokratie, Konzernen, Establishment und Medien und Iran selbst strategisch zu bedeutend, um einen Bruch mit dem Schah ernsthaft zu riskieren. Der Kleinkrieg zwischen „Blankoscheck“-Gegnern und Befürwortern setzte sich fort und erst die Revolution selbst sollte eine grundsätzliche Umkehr in den Beziehungen zwischen den USA und Iran erwirken. ${ }^{175}$

\footnotetext{
172 Vgl. MemCon, 29.9.76, FRUS 69-76, XXVII, S. 563ff.; JCS Chairman George Brown erwähnte 1976 in einem Interview die ,puzzling question of why [Iran] is building such a tremendous military force."; Brown beantwortete die Frage gleich selbst: "It makes you wonder whether he doesn't some day have \{sic\} visions of the Persian Empire. They don't call that the Persian Gulf for nothing."; zit aus JCS History Iran, S. 114f.

${ }_{173}$ Die Probleme mit Waffenexporten, die letztlich die Kapazitäten der Empfänger überstiegen, seien „real“, aber bedürften keines „, fundamental shift“ in der US-Politik. So der Tenor der Executive Summary vom November 1976 einer Interdepartmental Group, die die Antwort auf NSSM N ${ }^{\circ} 238$ formulierte. Offenbar war die Frage des Ausmaßes einer eigenen US-Militärpräsenz im Golf in der Studie weitaus bedeutender, wieder die omanische Alternative betonend. Vgl. JCS History Iran, S.117-20; für eine Kritik am nicht zuletzt durch US-Exporte angeheizten Rüstungswettlauf in der Nahostregion vgl. INR Research Study RS-57, 30.5.75, ,Persian Gulf", Lot 74 D 131, Records of Under Secretary of State for Political Affairs Joseph Sisco, 1951-76, Box 44.

${ }_{174}$ Eine ähnliche Schlussfolgerung bietet sich auch für die Nixon-Kissinger-Ford-Politik in der Levante an. Analog zu iranischen militärischen Hegemonie im Persischen Golf garantierte die US-Politik - allerdings im Einklang mit der Politik der Vorgänger - die militärische Präponderanz Israels. Hinzu kam die maßgeblich von Kissinger durchgesetzte Kehrtwende amerikanischer Mediationspolitik im breiteren Konflikt weg von einer umfassenden Konfliktlösung und hin zu einem Konfliktmanagement auf Basis der (wenn auch öffentlich nicht so signalisierten) Nichtinfragestellung territorialer Expansion Israels auf Basis der Waffenstillstandslinien nach dem Juni-Krieg von 1967, später nur zugunsten der arabischen Vormacht Ägyptens revidiert im Gegenzug für den Lagerwechsel im Kalten Krieg und einen Separatfriedensvertrag mit Israel und die resultierende Aufgabe der eigenen Führungsrolle in der arabischen Welt. Vgl. anschaulich YAQUB, Weight of Conquest; vgl. auch DAIGLE, Limits of Détente; der Hauptunterschied zur Levante aber ist das ,vitale“ Interesse der USA an der Golfregion und die Entschlossenheit aller amerikanischer Administrationen, der Errichtung eines regionalen Öl-Hegemons im Golf notfalls mit Waffengewalt entgegenzutreten.

${ }_{175}$ Zur Menschenrechtspolitik der Carter-Administration gegenüber Iran vgl. TRENTA, Champion of Human Rights; grundsätzlich setzte auch die Carter-Administration die vererbte Politik im Rüstungsbereich unvermindert fort, trotz aller Menschenrechts- und Rüstungskontrollrhetorik. Siehe BILL, Eagle and Lion, S. 226-33.
} 


\section{4. Vorzeichen der Revolution: Das Scheitern der amerikanischen Iranpolitik}

Ein wesentlicher Grund für die zunehmend kritische Perspektive auf den Schah in der USÖffentlichkeit war seine Rolle bei den Ölpreissteigerungen Anfang der 1970er Jahre. Im Anschluss an das Offtake Agreement vom Dezember 1966 und insbesondere der Haltung Irans während des kurzlebigen arabischen Ölembargos gegen den Westen im Kontext des Dritten Arabisch-Israelischen Krieges von 1967 hatte man seitens der US-Administration eigentlich mit etwas Ruhe an dieser Front gerechnet, zumal die iranische Öl-Förderung aufgrund der Umstände weitaus schneller angestiegen war als eigentlich vereinbart. Mohammad Rezā konnte somit zufrieden sein. Allerdings stieg der iranische Finanzbedarf analog zum aufgeblähten Militäretat und der Schah wiederholte seine Forderungen nach einem Anstieg der Einkünfte aus der Ölförderung nun im Jahresrhythmus. ${ }^{176}$ Nachdem im April 1968 eine noch einigermaßen ausgewogene Übergangsvereinbarung erreicht worden war, eskalierte schließlich das Verhältnis zwischen Iran und dem Konsortium im Folgejahr. Der Schah bestand nun auf einer Steigerung der Öleinnahmen auf eine Milliarde US-Dollar und verwies auf seine ambitionierten Entwicklungspläne. Angesichts der fortgesetzten Verweigerungshaltung des Konsortiums drohte er mit einer Nationalisierung der Hälfte der Konsortiumsoperationen und deren Übernahme durch die NIOC. Im Kern gab das Konsortium ein weiteres Mal nach und die Krise verschwand so rasch wie sie gekommen war - aber die Drohungen des Schahs hatten nun die Frage der Beteiligung der Förderländer in den Vordergrund gedrängt. Bald sollte sich diese Debatte auf den Gesamtkomplex zwischen Förderländern und Ölgesellschaften mit Zielrichtung auf graduellen Souveränitätswechsel hinsichtlich der Ressource Erdöl auswirken. ${ }^{177}$ Im Herbst 1970 nahm der Schah seine alte Drohung einer Nationalisierung wieder auf, vor allem animiert durch eine immer stärker werdende Dynamik zwischen Erdölförderländern und Gesellschaften, ausgelöst durch strukturelle Veränderungen des Marktes und politische Entwicklungen, vor allem die Machtübernahme von Mu'ammar al-

\footnotetext{
${ }^{176}$ Vgl. Solomon Action Memo, 12.10.67; Tehran \#2249/\#2702, 22.11./29.12.67, FRUS 64-68, XXXIV, S. 387ff., 394f., 395ff.; Iran und Venezuela unterliefen das arabische Embargo, das bald aufgegeben wurde. Vgl. ed. note, ebd., S. 472f.; die Nichtteilnahme am arabischen Boykott hatte allerdings auch den Stellenwert des iranischen Öls erheblich vergrößert. Undersecretary of State Eugene Rostow warnte die Ölgesellschaften: „As companies knew, even temporary boycott of Middle East oil, if it included Iranian production, would be catastrophic. "; State \#138990, 30.3.68, ebd., S. 405; Dowell Letter, 4.3.68, DDRS: CK3100344143; Solomon Memo, 26.3.68, DDRS: CK3100622521; Akins Letter, 18.3.68, PET 6 IRAN, CF 67-69; zum gestiegenen Bewusstsein der strategischen Achillesferse des Westens hinsichtlich der Abhängigkeit vom nahöstlichen Öl vgl. Interagency Study, Western Interest in Arab Oil', 27.12.67, encl. to Battle Memo, 18.1.68, ,SIG/MEMO \#52-2/9/68-IRG/NEA', Lot 70 D 263, SIG Files, 1966-1969, Box 2; mit den steigenden Devisenreserven Irans stieg zugleich das Interesse Washingtons an Investitionen in den USA selbst, insbesondere in amerikanische Staatsanleihen. Vgl. Fowler Memo, 12.6.68, DDRS: CK3100002994.

${ }^{177}$ Vgl. INR Research Memo RNA-34, 17.11.68, FRUS 64-68, XXXIV, S. 412f.; FO TELNO 2518 to Washington, 13.3.68, PREM 13/2169; CC(66)58, 17.11.66, fos 365, CAB 128/41; Stewart Memo, C(69)56, 20.5.69, CAB 129/142, TNA:PRO; INR Intel Note-361, 9.5.69; State \#76751, 15.5.69, FRUS 69-76, E-4, docs. 14, 15; Vgl. INR Research Memo RNA-26, 10.6.69, FRUS 69-76, XXXVI, S. 8-20; die CIA zeigte sich angesichts der ungewöhnlichen Schärfe des Disputs zunehmend besorgt. Vgl. CIA, NE Staff Note \#5-69 \{The Iranian Oil Crisis\}, 13.3.69; CIA, ONE Memo to DCI, 3.4.69, CREST: \#RDP79*014-1; Saunders Memo, 7.5.70, ,Iran, Vol. I, January 20, 69-May 31, 1970‘, NSCF, Country Files-Middle East, Box 601, RMNL; grundlegend jetzt zum Strukturwandel in der Ölindustrie DIETRICH, Oil Revolution, S. 228-61.
} 
Qadֵdāfī in Libyen. Die Machtbalance neigte sich nun mehr und mehr zugunsten der Förderländer: Die Vereinbarung von Teheran (Tehran Agreement) vom 14. Februar 1971 zwischen den OPEC-Mitgliedern am Persischen Golf und den Konzernen erwirkte erhebliche Preissteigerungen für das in der Region geförderte Erdöl sowie einen entsprechenden Anstieg der Royalties für die Förderländer - bis Mitte der 1970er Jahre sollten sich die Einnahmen verdoppeln, doch war die auf fünf Jahre angelegte Grundsatzvereinbarung bald wieder hinfällig. ${ }^{178}$

Die Ölpolitik Irans hatte sich in der Zwischenzeit grundlegend gewandelt. Anstelle der prowestlichen Haltung mit der Absicht, von der eigenen Mäßigung in der Preispolitik mittels Ausweitung der eigenen Förderquoten zum Nachteil der arabischen Produzenten zu profitieren, hatte man sich nun der OPEC-Kartellhaltung angepasst, mit dem Ziel kollektiv die Macht der Konzerne zu brechen und dem Westen die eigenen Preisvorstellungen zu diktieren. Im Vorfeld des Tehran Agreement waren es gerade die Iraner, die mit Nachdruck auf Preissteigerungen drängten. ${ }^{179}$ Der Hebel, den Mohammad Rezā nun ansetzte, war die 1979 auslaufende

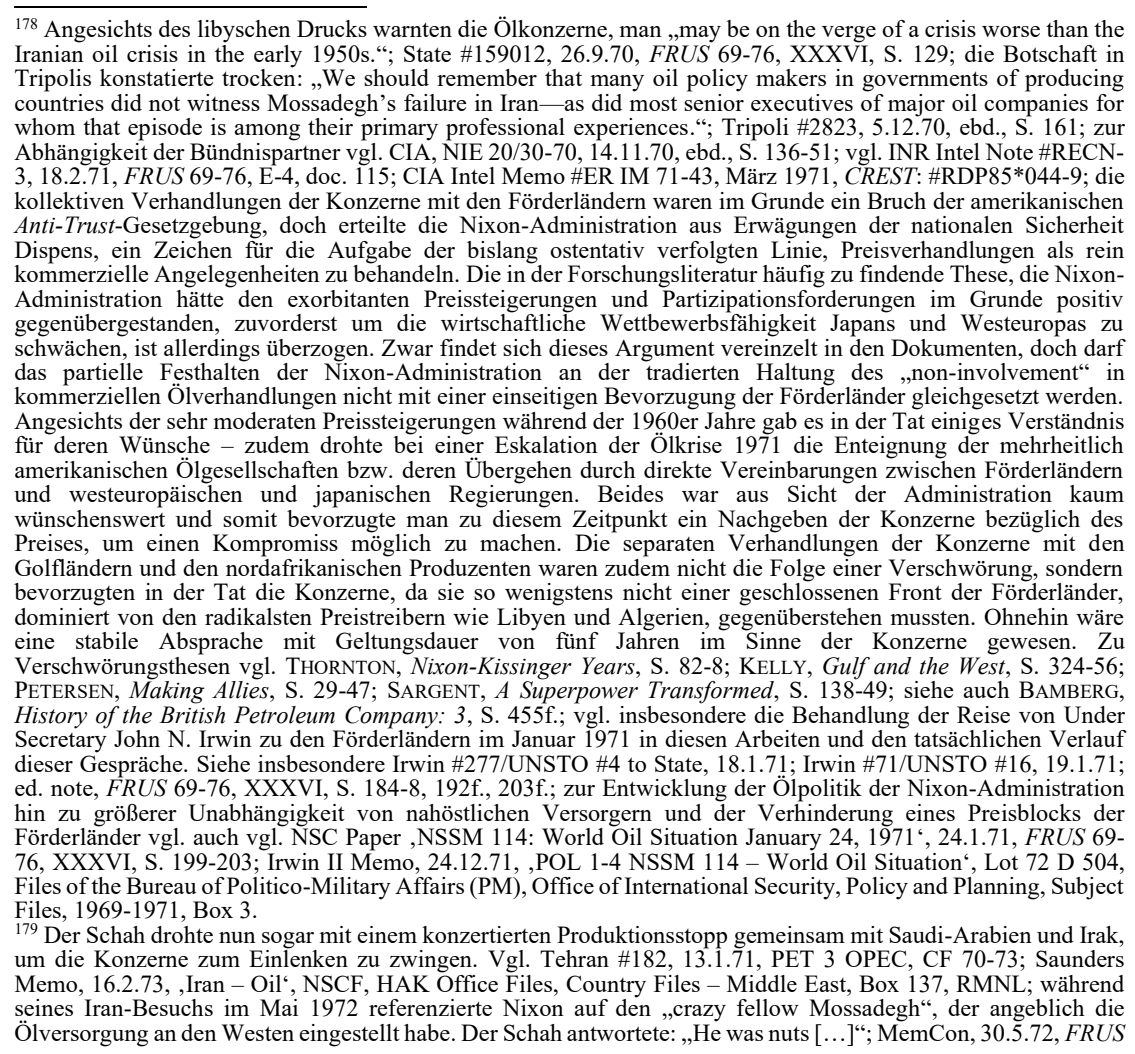


Konsortiumsvereinbarung, die eine grundsätzliche Neuregelung der Beziehungen zwischen dem iranischen Staat und den Gesellschaften vonnöten machen würde. Angefeuert von einer Konkurrenz mit den Saudis in Partizipationsfragen drängte Mohammad Rezā nun auf eine grundsätzliche Revision der bisherigen Ordnung und eine operative Übernahme sämtlicher upstream-Operationen - formal bereits seit 1954 unter souveräner Kontrolle - und auch der Raffination vor Ort durch den iranischen Staat und den direkten Verkauf des geförderten Öls an die Gesellschaften: ,[...] it would dramatically change the fundamental companygovernment relationship which has prevailed in essence from time immemorial to the present: day-to-day control by the major international oil companies of the sources of supply of most or all of the oil they require for their downstream operations. “180

Persönlichen Appellen Präsident Nixons im Januar 1973 zum Trotz hielt der iranische Monarch an seiner unnachgiebigen Haltung fest. ${ }^{181}$ Angesichts dieser Position - und der strategischen Bedeutung Irans für die USA - akzeptierte die Nixon-Administration letztlich den großen symbolischen Erfolg Mohammad Rezās in den Verhandlungen in St. Moritz Ende Februar 1973, ungeachtet der gefährlichen Präzedenz für die allgemeinen amerikanischen Ölinteressen in der sogenannten Dritten Welt. Ein Verhandlungsteam des Konsortiums stimmte der Aufhebung der Vereinbarung von 1954 und der Übergabe der Kontrolle aller Öloperationen in Iran an den Staat zu, den alten Traum Mosaddeg gon der Kontrolle des iranischen Öls durch die Nation konzedierend. Im Kern veränderte sich an den konkreten Operationen nur wenig, doch fanden sie nun seitens der Ölkonzerne formell im Rahmen eines umfassenden Kaufvertrags mit der NIOC statt. ${ }^{182}$

69-76, E-4, doc. 200; den „höheren“ Abschluss der Mittelmeerförderländer mit den Ölkonzernen im Abkommen von Tripolis im April beantwortete der Schah sogleich mit neuen Forderungen und dem Bruch seines eben erst gegebenen Versprechens, die Teheran-Vereinbarung nicht in Frage zu stellen. Die Konzerne kamen ihm ein weiteres Mal entgegen. Vgl. Tehran \#1935, 15.4.71, FRUS 69-76, XXXVI, S. 215 n5; in der Nixon-Administration war man sich der bevorstehenden fundamentalen Umwälzungen im Erdölmarkt bewusst und versuchte, eine Strategie zu entwickeln. Vgl. Study (by Akins), , The International Oil Industry Through 1980‘, 22.12.71, DNSA: IR00759; State Study, , The U.S. and the Impending Energy Crisis', 9.3.72, DDRS: CK3100516177.

${ }^{180}$ INR Intel Note RECN-36, 6.12.72, FRUS 69-76, E-4, doc 239; bevorzugt vom Schah für die post-1979-Zeit wurde anfangs eine Fortsetzung des bisherigen Arrangements bei gleichzeitiger Beteiligung Irans an downstreamOperationen (und Gewinnen) und einem umgesetzten Souveränitätswechsel in Fragen des Produktionsumfangs. Noch im Sommer 1971 wurde auf dieser Basis eine Vereinbarung mit dem Konsortium getroffen, die im Kern die ,exclusive production rights“ im Konzessionsgebiet auch für weitere 15 Jahre nach 1979 respektierte. Die Vereinbarung mit ihrer scheinbaren Durchbrechung der OPEC-Linie wurde sogleich als „major plus in our relations with the Middle East" interpretiert. Vgl. Tehran \#7307, 23.12.71, ebd., doc. 155; auch in FRUS 69-76, XXXVI, S. 239ff.; vgl. INR Intel Note RECN-17, 11.8.71, INR Intel Note RECN-15, 27.6.72; Flanigan Memo, 7.7.72, ebd., S. 216ff.; 298-301, 302ff.; vgl. CIA, ONE Memo \{, This Year's Oil Crisis: The Issue of Participation' $\}$, 24.3.72, CREST: \#RDP79*001-4; Flanigan Memo, 27.4.72, ,Visit of Richard Nixon to the Empire of Iran, 30-31 May 1972‘, Lot 74 D 416, Executive Secretariat, Briefing Books, 1958-1976, Box 164A; de facto bestand Mohammad Rezā damit auf einer 100\%-igen Beteiligung, im Gegensatz zu den Golfarabern, die sich gerade mit $25 \%$ jetzt und $51 \%$ im Jahr 1981 zufrieden gegeben hatten. Vgl. COOPER, Oil Kings, S. $91 \mathrm{ff}$.

${ }_{181}^{1}$ Mohammad Rezā machte dem CIA Station Chief seine unnachgiebige Haltung in der Frage unmissverständlich klar - offenkundig ein Signal an Washington intendierend. Vgl. ed. note; Saunders Memo, 30.1.73; Helms Memo, 7.2.73, FRUS 69-76, XXXVI, S. 382f., 396ff., 406ff.; CIA \#sanitized to DCI, 11.1.73, CIA-Helms; Hoskinson Memo, 2.2.73, FRUS 69-76, XXVII, S. 29ff.; vgl. Saunders Memo, 5.2.73, ,Iran, Vol. IV, 1 Sep 71-Apr 73‘, NSCF, Country Files-Middle East, Box 602, RMNL.

${ }^{182}$ Die Vereinbarung wurde am 24. Mai 1973 unterzeichnet - die formelle Übernahme von Management und operativer Tätigkeit der Ölindustrie erfolgte bereits am 21. März. Vgl. NSC Staff Paper, 16.2.73; FRUS 69-76, XXXVI, S. 411-7; vgl. Rush Memo, 1.3.73, FRUS 69-76, XXVII, S. 34f.; Rush Memo, 1.3.73, PET 6 IRAN, CF 
Der beschleunigte Souveränitätswechsel von Konzernen zu Regierungen der Ölförderländer erleichterte Versuche, die Preisspirale weiter nach oben zu drehen. Der saudische Ölminister Yamān̄̄ bezeichnete das Tehran Agreement bereits im September 1973 als ,either dead or dying“. ${ }^{183}$ Der Vierte Arabisch-Israelische Krieg (auch Oktoberkrieg, Jom-Kippur-Krieg bzw. Ramadankrieg genannt) im Oktober 1973 und der Einsatz der sogenannten „Ölwaffe“ durch die arabischen Staaten löste sodann einen weiteren, in seiner Reichweite zuvor unvorstellbaren Preisanstieg aus. Hier manifestierte sich nun die neue Rolle Irans als wichtigster Preistreiber. Bereits kurz nach Ausbruch des Krieges stieg der Ölpreis um $70 \%$ auf nun etwas über $\$ 5$ per Barrel. Noch dramatischer aber war die vor allem auf Betreiben Mohammad Rezās beschlossene Preissteigerung im Dezember 1973 um über 100\% auf nunmehr \$11,60. Triumphal verkündete der Schah anschließend auf seiner Pressekonferenz in Wien den Industriemächten, es sei nun an der Zeit für sie, den Gürtel enger zu schnallen. ${ }^{184}$ Der gewaltige Preisanstieg - Ölimportkosten für Westeuropa stiegen über \$33 Milliarden, die der USA \$16 Milliarden ${ }^{185}$ - verursachte den „Ölpreis-Schock“ in der industrialisierten Welt und war ein beitragendes Element einer Wirtschafts- und Sinnkrise in westlichen Hauptstädten, die bis in die 1980er Jahre anhalten sollte.

Wenig überraschend angesichts der dramatischen Folgen der Ölkrise entstanden unterschiedliche zeitgenössische Interpretationen, die aber alle die Behauptung teilten, die USA hätten im Arkanen mit den Ölförderstaaten kollaboriert, da beide Seiten - zu Lasten der übrigen westlichen Welt und der Entwicklungsländer - letztlich von den rasanten Preisanstiegen profitiert hätten. ${ }^{186}$ Zwar finden sich vereinzelt Belege für eine solche Idee. So teilte der NSCStab Kissinger mit, dass ,[...] sharp increases in European and Japanese energy prices would significantly help the international competitive position of the United States, and could

\footnotetext{
70-73; zum Sale-and-Purchase Agreement vgl. auch FERRIER, Iranian Oil Industry, S. 122ff.; zur „Winterresidenz“ des Schahs in Graubünden vgl. MEIER, Helvetiens guter Draht, S. 235-9.

${ }_{183}$ Armstrong Information Memo, 17.9.73, FRUS 69-76, XXXVI, S. 556; INR Research Study RECS-20, 19.9.73, PET 6 IRAN; Aldrich Memo, 15.11.73, POL 33 PERSIAN GULF, CF 70-73; Armstrong Memo, 11.10.73, DNSA: IR00836.

${ }^{184}$ Offensichtlich genoss der Schah den Moment in vollen Zügen und fügte an: „Eventually all those children of well-to-do families who have plenty to eat at every meal, who have their own cars, and who act almost as terrorists and throw bombs here and there, will have to rethink all these privileges of the advanced world."; zit. aus GRAHAM, Illusion of Power, S. 15; vgl. Tehran \#9087, 27.12.73, CFPF, 1973-79/Electronic Telegrams; State \#249539, 22.12.73, FRUS 69-76, XXVII, S. 162f;; vgl. ebd., S. 164 n2; Tehran \#9057, 26.12.73, FRUS 69-76, XXXVI, S. $774 \mathrm{ff}$; zur iranischen Ölpolitik in dieser Zeit vgl. auch FARMANFARMAIAN, Blood and Oil, S. 393ff.

${ }^{185}$ Vgl. CIA Paper \#ER IB 74-1, Januar 1974, FRUS 69-76, XXXVI, S. $789 \mathrm{f}$.

${ }^{186}$ Für ähnliche Behauptungen in Bezug auf das Tehran Agreement von 1971 vgl. oben S. 613, Anm. 178; vgl. auch THORNTON, Nixon-Kissinger Years, S. 302-16; die angebliche Servilität des Westens gegenüber den Ölproduzenten -, ,the most humiliating episode in the history of Western Christendom since the collapse of the last Crusade" - dient als Grundthema des gesamten Werkes von J.B. Kelly, genrestiftend für eine bis heute noch weit verbreitete Sichtweise. Siehe insbesondere KELLY, Gulf and the West, S. 378-457, Zitat auf 423; nicht unähnlich Kissinger während der Krise: „It is ridiculous that the civilized world is held up by 8 million savages.“; MemCon, 29.11.73, FRUS 69-76, XXXVI, S. 706; vgl. auch Atherton Jr. Memo, 20.10.74, DNSA: IR00900; vgl. auch die handschriftlichen Kommentare in Tehran \#9269, 2.11.74, DNSA: IR00907; für eine Neuinterpretation der Folgen der „Ölwaffe“vgl. jetzt GRAF, Arab Petropolitics; vgl. GARAVINI, Completing Decolonization; siehe auch die Beiträge in: BINI/GARAVINI/ROMERO, Oil Shock.
} 
therefore sharply improve our trade balance and overall balance of payments. “187 Dasselbe Memorandum nannte auch vielerlei Gründe für ein entschiedenes Eintreten der Administration gegen einen Ölpreisanstieg: Die bereits genannten Überlegungen bzw. öffentlichen Drohungen einer militärischen Antwort auf die „Ölwaffe“ waren durchaus mehr als ein Bluff und sind in der Tat ein starkes Indiz für die Wahrnehmung des Preisanstiegs als Bedrohung der eigenen Sicherheit und nicht etwa als kommerzielle Gelegenheit. Nixon selbst war zwar Mohammad Rezā eng verbunden - die immer wieder in Presse und Literatur kolportierte finanzielle Wahlkampfunterstützung für Nixon erscheint durchaus plausibel, wenngleich belastbare Belege noch ausstehen - und setzte sich wiederholt auch in Ölbelangen für ihn ein. Allerdings zeigte sich Nixon während der Ölkrise als den großen Ölgesellschaften nahe stehend, zur Frustration Kissingers und anderer Offizieller. ${ }^{188}$

Im Anschluss an den rasanten Preisanstieg zum Jahreswechsel 1973/74 versuchten die USA, aktiv auf die Regierungen der Förderländer einzuwirken, um wenigstens das erreichte hohe Niveau zu stabilisieren, was in der Tat für den Rest der Dekade gelang. ${ }^{189}$ Die Rolle Iran als einer der schärfsten Preistreiber unter den OPEC-Staaten hatte die bereits erwähnten Belastungen im bilateralen Verhältnis zu Washington zur Folge, doch sind die in der jüngeren Forschungsliteratur mitunter $\mathrm{zu}$ findenden Thesen, die USA hätten als Reaktion mit wirtschaftlichen Gegenmaßnahmen aktiv die Pahlawi-Herrschaft unterminiert, reichlich überzogen. ${ }^{190}$ Die negativen Auswirkungen auf die Allianz waren dennoch spürbar. Versuche, ein bilateralen Ölabkommens auszuhandeln, eine Barter-Vereinbarung zwischen den USA und Iran über den Austausch einer festgelegten Ölimportmenge mit US-produzierten Gütern bzw. sogar Waffen, in einer späteren Version mittels privater amerikanischer Unternehmen, fanden in den Jahren 1975 und 1976 statt, führten aber nicht zum Erfolg. ${ }^{191}$ Somit schwelten die

\footnotetext{
${ }^{187}$ Bergsten/Saunders Memo, 14.1.71, FRUS 69-76, XXXVI, S. 175; ein wichtiges Argument dagegen war allerdings die Sorge, die europäischen Konsumenten könnten dazu übergehen, die US-Konzerne zu übergehen und direkte Liefervereinbarungen mit den Produzenten anzustreben. Siehe ebd., S. $174 n 2$; vgl. TelCon, 5.6.75, State:HKTT.

${ }^{188}$ Im Februar 1970 sandte Nixon einen „Befehl“ an das Konsortium, die Fördermenge in Iran gemäß den Wünschen Mohammad Rezās zu steigern. Andernfalls drohte er mit Konsequenzen in der Ölimportfrage. Siehe FRUS 69-76, E-4, doc. $50 n 1$; eine weitere Ermahnung folgte im Oktober: ,tell these oil barons -American security is vitally involved."; ebd., doc. $89 n 1$; vgl. auch Flanigan Memo, 10.1.70, FRUS 69-76, XXXVI, S. 93-6; zur Besorgnis wegen Nixons „Oil Friends“" und deren versuchter Einflussnahme auf die US-Politik vgl. ed. note, ebd., S. $690-3$.

${ }_{189}$ Die Ursachen für die Stabilisierung des Ölpreises ab 1974 sind in der Tat vielfältig und haben mit dem Nachfrageeinbruch infolge der Rezession in den westlichen Industriestaaten, Steigerung der Produktion, aber auch Uneinigkeit zwischen den wichtigsten OPEC-Ländern zu tun. Auf dem OPEC-Treffen in Bali im Mai 1976 wurde die von Iran geforderte Steigerung ein weiteres Mal vertagt, offenbar infolge saudischen Widerstands. Vgl. State \#152797, 15.7.74; Tehran \#6142/\#8946/\#4921, 24.7.74/11.9.75/16.5.76; State \#267996, 30.10.76; Shah Letter, 1.11.76, FRUS 69-76, XXVII, S. 210ff., 215ff., 432ff., 525f., 569-72, 572ff.; vgl. ebd. S. 526 n4; State \#214124, 9.9.75, FRUS 69-76, XXXVII, S. 276ff.; vgl. Lord Memo, 21.9.74, DNSA: IR00893.

190 So die These von COOPER, Showdown at Doha, der von einer geheimen Absprache zwischen Riad und Washington ausgeht, die das OPEC-Treffen in Doha vom Dezember 1976 unterlaufen habe mit dem Ziel, die Position des Schahs zu schwächen. Die tatsächlichen Beweggründe waren allerdings komplexer, stehen aber außerhalb des Fokus dieser Arbeit. Vgl. aber State \#309057, 22.12.76, FRUS 69-76, XXXVII, S. 397-401; vgl. auch PAINTER, Oil in the 1970s.

${ }^{191}$ Ein wichtiges US-Interesse dahinter war, mittels eines indirekt eingebauten Rabatts die OPEC-Preisstruktur zu sprengen. Im Kontext der erklärten Absicht zum Aufbau einer Strategic Petroleum Reserve erfuhr die Idee zwischenzeitlich erneuten Auftrieb - man beachte die Parallelen zum ähnlichen Vorschlag des Schahs, wenngleich unter umgekehrten Voraussetzungen, im Jahr 1969. Siehe oben S. 566, Anm. 14; Hauptgründe für das Scheitern
} 
Streitigkeiten über den angemessenen Ölpreis fort und führten auf beiden Seiten zu Verstimmungen. Tatsächlich verfügte Washington über Geheimdienstinformationen, die belegten, wie unauthentisch in der Tat das großspurige Auftreten Mohammad Rezās im Kontext der rapiden Preiserhöhungen gewesen war. Intern, so berichtete eine CIA-Quelle aus dem Umfeld des Herrschers, sorgte sich der Schah in der Tat über mögliche Vergeltungsmaßnahmen der USA. Offenbar befürchtete er, dass die USA im Gegenzug die „Blankoscheck“-Politik revidieren und den Waffenexport nach Iran begrenzen könnten. Neben den durchaus auch mit Billigung der US-Administration in die Welt gesetzten und in der Tat nicht unbegründeten Mutmaßungen über Planungen eines eventuellen amerikanischen Militäreinsatzes im Golf zur Sicherung der Ölversorgung gaben insbesondere Spekulationen über geheimdienstliche verdeckte Operationen Anlass zur Verunsicherung. Ein Artikel des Economist Anfang 1974 kommentierte Gerüchte in Washington, Nixon habe die Absicht „to unleash the CIA“ mit dem Ziel, fügsamere Herrscher in den Golfstaaten zu installieren. Erinnerungen an Dr. Mosaddeg seien , ,...] very much alive these days in Washington. "192 Tatsächlich sollte der Schah kurz vor seinem Tod seinen Sturz in erster Linie auf seine unabhängige Ölpolitik zurückführen, die letztlich eine Verschwörung von äußeren und inneren Feinden gegen seinen Herrschaft herbeigeführt habe. ${ }^{193}$ Weder gab es ernsthafte Absichten für solche Operationen in Washington zu dieser Zeit noch verfügten US-Geheimdienste Mitte der 1970er Jahre über entsprechende Kontakte und strategischen Einfluss in Iran, um auf einen Machtwechsel in Teheran hinzuwirken. Bei allen Neigungen zu Verschwörungstheorien und der immer wieder in den Dokumenten belegbaren, ausgeprägten Paranoia Mohammad Rezās ist hier durchaus auch der Versuch zu sehen, sich im Nachgang des eigenen Scheiterns wenigstens als Held und Verteidiger der iranischen Nation zu stilisieren - bis heute eine bevorzugte Strategie kaiserlicher Apologeten.

Bezeichnend für das Festhalten an der engen Allianz mit dem Pahlawi-Iran war die Fortsetzung der Rüstungskooperation. Doch auch diese blieb von den Spannungen nicht verschont, die sich

\footnotetext{
waren die Unvereinbarkeit eines solchen Geschäfts mit den rechtlichen Rahmenbedingungen in den USA für den Ölimport und die aus einem solchen Deal resultierende Diskriminierung der Saudis. Vgl. Robinson Memo, 13.5.75; Hormats/Oakley Memo, 6.6.75; Kissinger Memo, 15.8.75; Kissinger Memo to Ford, o.D., attached to Hormats Memo, 30.12.75; MemCon, 29.3.76; Robinson Action Memo, 1.10.76, FRUS 69-76, XXVII, S. 367ff., 401ff., 419-22, 453-7, 516ff., 565f.; vgl. ebd. 406 n4; im Kontext des Verkaufs der 150 F-16-Kampfflugzeuge im Juli 1976 kam es offenbar zu entsprechenden ,privaten“ barter deals. Vgl. Atherton Action Memo, 29.7.76, ebd., S. 533-6; Zarb Memo, 13.1.76, FRUS 69-76, XXXVII, S. 329ff.; für ähnliche Überlegungen mit Bezug auf SaudiArabien vgl. Scowcroft/Cooper Telegram, 1.3.74, FRUS 69-76, XXXVI, S. 920ff.; TelCon, 22.12.75, State:HKTT. ${ }_{192}$ Zit. aus FRUS 69-76, XXVII, S. $168 n 2$ \{Hervorheb. v. Verf.\}; vgl. auch State \#TOSEC431, 31.10.74, ebd., S. $257 \mathrm{f}$.

${ }^{193}$ Mohammad Rezās im Exil verfasste Memoiren verwiesen auf fortschreitende westliche Absichten ,,to clip my wings“ seit den Tagen Mosaddeġs. Nach der Ölkrise habe sich dann eine ,strange confluence of interests international oil consortium, the British and American governments, the international media, reactionary religious circles in my own country, and the relentless drive of the Communists" gebildet, die letztlich die Hintermänner hinter den Aufständen von 1978 gewesen seien. Ähnlich bizarr die Behauptung, Franzosen und Deutsche hätten seinem Sturz auf dem Gipfel von Guadeloupe 1979 in einem „Yalta of the Mideast“ zugestimmt. Siehe PAHLAVI, Answer to History, S. 22f., 171; ausführlicher zum angeblichen Komplott die Schwester des Schahs in PAHLAVI, Jamais Résignée, S. 55-80; eine kritische iranische Stimme zur Verschwörungstheorie bei AMUZEGAR, Dynamics, S. 79-96.
} 
indirekt aus der Ölkrise ergaben. Tatsächlich mehrten sich bereits Ende 1973 die Stimmen im DOD, die quasi als Ausgleich für die höheren amerikanischen Ölimportkosten zugunsten eines Aufschlags auf die Preise der iranischen Waffenkäufe plädierten. ${ }^{194}$ Offenkundig konzentrierte sich gerade im Pentagon die amerikanische Verstimmung über die rasante Ölpreissteigerung. Als sich der Schah persönlich bei Verteidigungsminister James Schlesinger über die Preissteigerungen zwischen 50 und 300\% in den letzten Jahren beschwerte, erwiderte dieser nur lapidar ,[...] that perhaps the growth in fuel prices had an important bearing on such inflation. “195 So stieg der Preis für einen Zerstörer der Spruance-Klasse von maximal \$120 Millionen im Jahr 1973 auf \$240 Millionen im Folgejahr und schließlich \$340 Millionen im Herbst 1975. Nicht zu Unrecht sahen die Iraner eine willkürliche Preiserhöhung seitens des DOD als Vergeltung für die iranische Rolle beim Anstieg der Ölpreise, aber auch als subtile Steuerung bzw. Regulierung der iranischen Aufrüstung, somit wieder ein Unterlaufen des „Blankoschecks“. 196 Der stellvertretende iranische Verteidigungsminister General Hasan Toufānijān, seit 1963 die Waffeneinkäufe im Auftrag des Herrschers koordinierend, warnte Anfang 1976 Donald Rumsfeld, Schlesingers Nachfolger im Amt, Iran sei angesichts sinkender Öleinnahmen und der rasant steigenden Waffen- und Ausrüstungspreise gezwungen, einen großen Teile seiner Bestellungen zu stornieren. ${ }^{197}$ Angesichts der sich immer antagonistischer gestaltenden Beziehungen in der Militärkooperation griff Mohammad Rezā Ende 1976 schließlich auf eine bewährte Drohgebärde zurück: Ein weiteres Mal ging Iran nun eine umfangreiche Liefervereinbarung für Kriegsgerät mit der UdSSR ein, in Abweichung von früheren Deals dieses Mal auch höher entwickelte Systeme wie die schultergestützte $S A-7$ Flugabwehrrakete und Flakpanzer vom Typ ZSU-23-4 einschließend. ${ }^{198}$ Die Kooperation zwischen den USA und Iran auf dem militärischen Gebiet, lange Jahre der Kitt der gegenseitigen Beziehungen auf Grundlage des „Blankoschecks“, wurde Mitte der 1970er Jahre mehr und mehr zu einer Belastung.

Eine weitere Sphäre, in der sich das wachsende Misstrauen zwischen den Verbündeten bemerkbar machte, war das zivile Nuklearprogramm Irans. Die ,alte“ Vereinbarung zwischen den USA und Iran von 1957 im Rahmen des Atoms for Peace-Programmes war nicht mehr

\footnotetext{
${ }^{194}$ Siehe vor allem die Kommentare Schlesingers und Moorers in FRUS 69-76, XXVII, S. 164 n3.

195 MemCon, 16.5.75, ebd., S. 380.

196 Vgl. Helms Backchannel \#228 to Rumsfeld, 17.11.75, ebd., S. 442f.; im September 1976 wurde der Schah informiert, dass er für die ersten $160 \mathrm{~F}$-16-Kampfflugzeuge Gesamtkosten von etwa \$3,87 Milliarden zu erwarten habe, nachdem ihm General Dynamics in früheren Absprachen eine Gesamtrechnung von \$2,14 Milliarden für alle 300 gewünschten Maschinen zugesagt hatte. Zwar erklärte sich die Divergenz in Teilen als ein Missverständnis, doch waren die Preissteigerungen dennoch fraglos exorbitant. Vgl. ebd., S. 562 n5; vgl. Granger Memo, 3.9.76, ebd., S. $561 \mathrm{ff}$; so z.B. auch Admiral Moorer: „Maybe we should raise the price of our stuff to the Shah"; MemCon, 28.12.73, ISCAP.

${ }^{197}$ Vgl. MemCon, 19.1.76, FRUS 69-76, XXVII, S. 464-72; zur weitverbreiteten Korruption im Zusammenhang mit den Rüstungsgeschäften vgl. ed. note, ebd., S. 592f.; COOPER, Oil Kings, S. $283 \mathrm{ff}$.

${ }^{198}$ Der Schwenk hin zu sowjetisch entwickelten Flugabwehrsystemen war offenbar nicht zuletzt eine Reaktion auf amerikanisches Zögern, die ebenfalls schultergestützte FIM-92 Stinger als Teil der angedachten militärischen coproduction in Iran herzustellen. Vgl. Oakley Memo, 10.12.76, FRUS 69-76, XXVII, S. 583ff.; Toufānijān erwähnte explizit die Lieferung von Ersatzteilen und Zusatzausrüstung in großem Umfang. Man sein nicht mehr „hostage to spares and support as in past“, eine offenkundige Anspielung auf mangelndes amerikanisches Entgegenkommen in der Vergangenheit. Vgl. Tehran \#1226, 9.12.76, CFPF, 1973-79/Electronic Telegrams.
} 
zeitgemäß, als Mohammad Rezā Anfang 1974 überaus ambitionierte Pläne für ein ziviles Nuklearprogramm verkündete. Nach einer sehr schleppenden Anlaufphase der bisherigen Nuklearforschung in Iran - der von den USA gelieferte 5-Megawatt-Forschungsreaktor für die Teheraner Universität ging erst Ende 1967 kritisch - plädierte der Schah nun angesichts des für das Ende des Jahrhunderts vorausgesagten Versiegens der Ölförderung für den Aufbau einer umfassende Nuklearindustrie mit einer Gesamtkapazität von 23.000 Megawatt Energie. Charakteristisch war wieder einmal der rasante Aufbau von Institutionen und Personal ungeachtet begrenzter Vorkenntnisse und unzureichender technologischer Kompetenz - die erst 1974 gegründete Atomic Energy Organization of Iran (AEOI) sollte nur zwei Jahre später bereits über ein Budget in Höhe von über \$1 Milliarde verfügen. Das ambitionierte zivile Nuklearprogramm wurde sogleich in Washington als potentielles Kernelement der eben erst gegründeten Joint Economic Commission mit dem Ziel der Intensivierung der wirtschaftlichen Zusammenarbeit identifiziert. ${ }^{199}$

Die atemberaubenden Profitaussichten angesichts des angedachten Umfangs des iranischen Programms und die neue westeuropäische Konkurrenz im zivilen Nukleargeschäft nach Auflösung des amerikanischen de facto-Monopols in diesem Bereich mahnte eigentlich zur Eile. Allerdings gab es auch hier wieder einflussreiche US-Offizielle, die zur Zurückhaltung mahnten, da sie den Beteuerungen des Schahs, keine militärischen Ambitionen im nuklearen Feld zu verfolgen, keinen Glauben schenkten. Angesichts des als Besessenheit zu charakterisierendem Verhältnis Mohammad Rezās zu modernem Kriegsgerät war diese Zurückhaltung durchaus begründet - in Reaktion auf die indische „Peaceful Nuclear Explosion“ im Mai 1974 gab der Schah zudem entsprechenden Mutmaßungen neue Nahrung, als er in einem Interview mit einem französischen Magazin auf die Frage, ob Iran in der Zukunft Nuklearwaffen besitzen würde, antwortete: „Certainly, and sooner than is believed [...].“200 Zwar beteuerten iranische Offizielle im Anschluss, es habe sich um ein Fehlzitat gehandelt. Allerdings stellte die CIA zeitgleich in einer Einschätzung zukünftiger Proliferateure fest:

If he $\{$ Mohammad Rezā; R.P. $\}$ is alive in the mid-1980s, if Iran has a full-fledged nuclear power industry and all the facilities necessary for nuclear weapons, and if other countries have proceeded with weapons development, we have no doubt that Iran will follow suit. ${ }^{201}$

Das DOD gab zu bedenken, dass aus einem zivilen Nuklearprogramm in der von Mohammad Rezā anvisierten Größenordnung ein jährlicher Produktionsumfang an waffenfähigem

\footnotetext{
${ }_{199}$ Vgl. Seaborg Letter, 20.2.69, DDRS: CK3100566190; Interdepartmental Working Group Paper, 25.4.74, FRUS 69-76, XXVII, S. 183-92; State \#73527, 11.4.74, CFPF, 1973-79/Electronic Telegrams; Atherton/Sievering Memo, 6.12.74, DDRS: CK3100646281.

${ }^{200}$ Paris \#15305, 24.6.74, NSArch EBBook No. 268, doc \#1a; Paris \#15445, 25.6.74; Tehran \#5192, 25.6.74, NSArch EBBook No. 521, docs. \#1, \#2; zur europäischen Konkurrenz siehe jetzt z.B. RoMBERG, Atomgeschäfte, S. 121-76.

${ }^{201}$ CIA, SNIE 4-1-74, 23.8.74, S. 38; verfügbar unter http://www2.gwu.edu/ nsarchiv/NSAEBB/NSAEBB240/ snie.pdf; vgl. auch Tehran \#6939, 17.7.75, NSArch EBBook No. 268, doc. \#7.
} 
Plutonium äquivalent zu 600-700 Nuklear-Sprengköpfen resultieren werde. ${ }^{202}$ Befürchtungen hinsichtlich einer iranischen nuklearen Waffenoption konkurrierten Mitte der 1970er Jahre mit der Wünschbarkeit einer kommerziellen Beteiligung von US-Unternehmen an der zu erwartenden „Bonanza“. Die Widerspenstigkeit des Kongresses bezüglich Nuklearexporten, gerade nach dem Überschreiten der nuklearen Schwelle durch Indien bei Verwendung eines von Kanada gelieferten Reaktors, war auch nicht durch Verweis auf die Unterzeichnung und Ratifikation des Atomwaffensperrvertrags (Treaty on the Non-Proliferation of Nuclear Weapons; NPT-Vertrag) durch Iran 1968/70 und die gemeinsame ägyptisch-iranische diplomatische Initiative zugunsten einer kernwaffenfreien Zone im Nahen und Mittleren Osten zu besänftigen. Das Bestehen auf strikten Regeln bezüglich der Kernmaterialüberwachung (safeguards) in einer zukünftigen Nuklearvereinbarung mit Iran wurde von der US-Botschaft als unangebracht und diskriminierend gebrandmarkt. Dennoch beharrte die Administration gegenüber Iran auf Restriktionen, die über die etablierten IAEA-Bestimmungen weit hinausgingen, gerade was das in der Produktion anfallende und potentiell waffenverwendbare Plutonium anging, ganz offenkundig auf Blockierung bzw. wenigstens Erschweren potentieller iranischer Atomwaffenambitionen abzielend. ${ }^{203}$ Entsprechend kontrovers gestalteten sich die Verhandlungen im Herbst 1975 - die Iraner verweigerten eine Behandlung als „Bürger zweiter Klasse“. In Washington hoffe man noch eine ganze Weile den Schah zu überzeugen, ein Beispiel für die Welt zu setzen - ein ,major act of nuclear statesmanship“ -, und auf Wiederaufbereitung im nationalen Rahmen zu verzichten. Im Mai 1976 standen sodann die Konturen einer bilateralen Vereinbarung über die zivile Nuklearkooperation, verschärfte safeguards, aber auch eine, wenn auch teilweise eingeschränkte Option auf eine nationale Aufbereitungsanlage in Iran enthaltend. Tatsächlich kamen die Verhandlungen erst 1978 zum Abschluss, doch kam die zugesagte Lieferung von acht Reaktoren an Iran infolge der Revolution nicht mehr zustande. ${ }^{204}$ Das augenfällige Misstrauen der Amerikaner hinsichtlich der nuklearen Absichten Irans stellte Mitte der 1970er Jahre einen zusätzlichen negativen Faktor für die ohnehin belasteten Beziehungen dar. Das Ausbleiben einer vertraglichen

\footnotetext{
${ }^{202}$ Zit. aus Martin (ISA) Memo, Juni 1974, ebd., doc. \#2; zeitgenössische Analysten glaubten in der Regel ebenfalls an militärische Absichten hinter Irans Atomprogramm. Vgl. MARTIN, Future Strategic Role, S. 244ff.; QUESTER, Shah and the Bomb; vgl. Hudson Institute Briefing Notes [Lewis A. Dunn], ,Iran and Nuclear Weapons`, 29.7.75, DDRS: CK3100646295.

${ }^{203}$ Tatsächlich sahen die ersten Entwürfe ähnliche Safeguard-Regeln wie in dem zu dieser Zeit angedachten Nukleargeschäft mit Ägypten und Israel vor, obgleich letztere nicht Unterzeichner des NPT waren. Helms prophezeite, der Schah würde sich einfach anderen Lieferstaaten zuwenden. Vgl. Tehran \#9039, 25.10.74, CFPF, 1973-79/Electronic Telegrams; vgl. Atherton Action Memo, 19.10.74; Atherton/Sievering Action Memo, 6.12.74; NSSM N²19 \{U.S.-Iran Agreement on Cooperation in Civil Uses of Atomic Energy\}, 14.3.75; NSDM N²92 \{U.S.-Iran Nuclear Cooperation\}, 22.4.75, FRUS 69-76, XXVII, S. 251ff., 274-7, 332, 335f.; vgl. ebd., S. 204 n3; wieder einmal war das Misstrauen im DOD am stärksten. Vgl. Schlesinger Memo, 25.4.74, ebd., S. 336f.; Ingersoll Memo, 18.4.75, DDRS: CK3100646286; vgl. BURR, Nuclear Negotiations, S. 23f.

${ }^{204}$ Die Amerikaner versuchten im Grunde ein eigenes Veto-Recht über die zukünftige Wiederaufbereitung von Nuklearmaterial durchzusetzen, das im Zuge eines Deals geliefert werden sollte. Umwege für ein solches Veto waren eine buy back-Option oder die Errichtung einer multinationalen Wiederaufbereitungsanlage in Iran, um exklusive nationale Kontrolle zu verhindern. Vgl. Scowcroft Memos, 26.1./4.2.76, Kratzer/Atherton Action Memo, 26.3.76, FRUS 69-76, XXVII, S. 473-7, 488ff., 502-9; Scowcroft Memo, 4.2.76, NSArch EBBook No. 268, doc. \#11; State \#125971, 17.5.78, NSArch EBBook No. 521, doc. \#9; vgl. Briefing Paper, ,The U.S.-Iran Nuclear Energy Agreement', 20.10.78, DNSA: IR01605.
} 
Vereinbarung sei ein „serious irritant in our relations with Iran“, schrieb Sicherheitsberater Brent Scowcroft im April 1976 an Präsident Ford. ${ }^{205}$

Unmittelbar verbunden mit den Spekulationen über ein zukünftiges iranisches Atomwaffenprogramm war das augenfällige Interesse des Schahs an Boden-Boden-Raketen mit entsprechender Tragfähigkeit. Nachdem eine Anfrage bezüglich der amerikanischen Kurzstreckenrakete MGM-52 Lance abschlägig beschieden worden war - ein weiterer Beleg für die fortbestehenden Beschränkungen trotz „Blankoscheck“ - beauftragte er offenbar Toufānijān, in Moskau bezüglich der SCUD anzufragen. Irritiert notierte man im NSC, der Schah sei offenbar „willing to pay a considerable political price to obtain a missile system." ${ }^{\text {"206 }}$ Botschafter Helms warnte diesbezüglich vor Geheimabsprachen zwischen Israel und Iran, um die Widerspenstigkeit des US-Kongresses bezüglich der Weitergabe bestimmter Waffensysteme zu umgehen. Das Gesprächsprotokoll eines Treffens zwischen Toufānijān und Außenminister Mosche Dajan in Israel im Juli 1977 - das Dokument war ebenfalls Teil der Beute der Botschaftsbesetzer von 1979-81 - erwähnte ein gemeinsames „Geheimprojekt“, offenbar eine Boden-Boden-Rakete mit einer Nutzlast von 750 Kilogramm, somit auch mit einem Nuklearsprengkopf bestückbar. ${ }^{207}$ Weitere Forschungen über diese enge Kooperation zwischen Israel und Iran und eine mögliche Beteiligung Irans an der engen Nuklearkooperation zwischen der Apartheid-Regierung in Südafrika und Israel stehen noch aus. Wenig bekannt ist zudem bislang über die geheimdienstliche Dimension der Nuklearbeziehungen zwischen Iran und den USA. Eine populäre Studie des US-Journalisten Bob Woodward erwähnt z.B., dass bereits zu Zeiten des Schahs die Beobachtung militärischer Nuklearambitionen eine Schwerpunktaufgabe der CIA vis-à-vis Irans gewesen sei, eine weitere interessante Parallele zur Gegenwart. ${ }^{208}$

\footnotetext{
${ }^{205}$ Scowcroft Memo, 19.4.76, FRUS 69-76, XXVII, S. 520; vgl auch NSDM N³24 \{Negotiation of a Nuclear Agreement with Iran\}, 20.4.76, ebd., S. 522f.; für die Unzufriedenheit mit dem Deal vgl. die Kommentare und Zeichnungen am Textrand in Tehran \#7886, 3.8.76, in: NSArch EBBook No. 268, doc. \#22.; Stanford Research Institute Report for DIA, ,Nuclear Proliferation and Iran: Net Assessment (A Case Study) ‘, August 1977, DOD FOIA Reading Room; für eine Betonung der Widersprüchlichkeiten des westlichen Ansatzes gegenüber dem iranischen Nuklearprogramm vgl. HAMBLIN, Nuclearization of Iran; PATRIKARAKOS, Nuclear Iran, S. 16-89; HOMAYOUNVASH, Iran and the Nuclear Question, S. 1-98.

${ }^{206}$ Oakley Memo, 10.12.76, FRUS 69-76, XXVII, S. 584; zur Vereinbarkeit mit dem „Blankoscheck“" vgl. Michaud Memo, 10.12.73, DEF 12-5 IRAN, CF 70-73; Briefing Paper, ,Military Supply: Lance Missile‘, Mai 1975, DDRS: CK3100001892.

${ }^{207}$ Helms spekulierte hier über den Hintergrund des Geheimbesuchs des israelischen Ministerpräsidenten Rabin in Iran im Juli 1976. Trotz intensiver Bemühungen sei es ihm nicht gelungen, Details über die Themen des Besuchs und des anschließenden Gegenbesuchs von Toufānijān in Israel zu erfahren. Vgl. Tehran \#7765, 1.8.76, FRUS 6976, XXVII, S. 536-9; Dajan sprach explizit von einer ,double purpose“-Rakete und erwähnte die denkbare Bestückung mit einem ,nuclear head“" und bezog sich auf „Americans' sensitivity to the introduction of the kind of missiles envisaged in the joint project.“; MemCon [Dajan, Toufānijān], 18.7.77, Asnād 19, S. 5 des Dokuments. Ein weiteres Dokument erwähnt einen Raketentest während des Besuchs Toufānijāns mit eben dieser Rakete. Vgl. MemCon [Toufānijān, Weizman u.a.], 18.7.77, ebd., o.S.; zur vertieften Nuklearkooperation mit Indien vgl. State \#53639, 10.3.77, Tehran \#5716, 12.7.74, CFPF, 1973-79/Electronic Telegrams.

${ }_{208}$ Die Botschaft schenkte wenig überraschend den iranischen Beteuerungen über die rein friedlichen Absichten hinter dem Programm Glauben, ein weiterer Ausdruck der Localitis. In einer Fundamentalkritik des NIE vom Mai 1975 zu Iran beschwerte sich die Botschaft, die Einschätzung der Geheimdienste sei ,,too dramatic in predicting Shah will attempt to acquire nuclear weapon technology." Die latente Kapazität hinter dem nuklearen Aufbau sei zwar nicht zu bestreiten, diene aber höchstens als Rückversicherung im Falle eine nuklearen Rüstungswettlaufs in der Region. Siehe Tehran \#5850, 19.6.75, FRUS 69-76, XXVII, S. 408. Einschätzungen von der CIA und dem DOD zum iranischen Programm aus den Jahren 1970, 1974 und 1976 wurden noch in den 2010ern die Freigabe
} 
Trotz der bilateralen Belastungen, die sich aus der Ölkrise und den wenig zufriedenstellenden Nuklearverhandlungen im gegenseitigen Verhältnis ergaben, darf die im Grundsatz positive Bewertung der Liaison mit dem Iran Mohammad Rezās in der Wahrnehmung der großen Mehrheit US-amerikanischer Offizieller, aber auch der Öffentlichkeit, nicht unterschätzt werden, wenngleich im letzteren Fall mit abnehmender Tendenz. Gerade vor dem Hintergrund des grotesk anmutenden Scheiterns amerikanischer Nation-Building-Anstrengungen in Südostasien erschien Iran mit seiner pro-westlichen Außenpolitik, seiner im Entstehen begriffenen Großmachtrolle in der Nahostregion, und seiner fast schon euphorischen Adaption an das westlich-kapitalistische Gesellschafts- und Wirtschaftsmodell wie eine Erfolgsgeschichte par excellence. Während iranische Aufrüstungspolitik und Großmannssucht wenig überraschend kaum Anklang fanden, waren im Gegensatz dazu die wirtschaftlichen Erfolge und die von oben fortwährend beschleunigte Modernisierungspolitik lange Zeit aus Sicht westlicher Beobachter geradezu ein Referenzpunkt, auch und gerade mit Sicht auf die vielen gescheiterten staatssozialistischen Experimente in der Dritten Welt. Den Ritterschlag hatte Iran von keinem Geringeren als Walt Rostow selbst bereits im Sommer 1968 erhalten. Der Vater der modernen Entwicklungsökonomie, inzwischen Nationaler Sicherheitsberater, verortete die wirtschaftliche Entwicklung Irans entlang der von ihm populär gemachten Wachstumsstadien: Iran sei kurz davor, die Take-Off-Phase abzuschließen und ins (kapitalistische) Reife-Stadium selbstragenden Dauerwachstums vorzudringen. ${ }^{209}$ Nicht zu bestreiten war indes, dass das ohnehin beeindruckende Wirtschaftswachstum nach 1963 am Ende der Dekade eine weitere Beschleunigung erfahren hatte. Die rapide steigenden Öleinnahmen hatten auch das privatwirtschaftliche Engagement neu belebt, das am Ende der Dekade mehr als drei Viertel über den Vorhersagen liegen sollte. Die iranische Regierung kalkulierte nun mit einem jährlichen Wachstum von nahezu 10\% für den 1968 beginnenden Fünfjahresplan, der bis 1973 öffentliche und private Investitionen in Höhe von \$11 Milliarden vorsah. $^{210}$

Die Selbstwahrnehmung der wirtschaftlichen Zukunft korrespondierte alsbald mit den militärisch-strategischen Großmachtplänen. Mohammad Rezā orientierte seine wirtschaftlichen Visionen analog zum Fortschritt der auf diesem Gebiet erfolgreichsten nichtwestlichen Macht: Japan. Der nächste Fünfjahresplan, beginnend 1973, sollte eine

verweigert. Siehe Reference Nos. 2011-095, 2010-070 und 2009-054, ISCAP; zu den CIA-Schwerpunkten vgl. WoODWARD, Veil, S. 82f.; KiBAROGLU, Iran's Nuclear Ambitions, S. 230f., beschreibt eine weitgehend kooperative bis willfährige Haltung der USA zum Nuklearprogramm des Schahs. Angesichts der Quellenlage ist diese Position unhaltbar.

${ }^{209}$ Vgl. MemCon, 13.6.68, FRUS 64-68, XXII, S. 530f.; vgl. JIC(B)(69)2, 6.6.69, CAB 188/3, TNA:PRO.

${ }^{210} \mathrm{Vgl}$. Record of NSC Interdepartmental Group for Near East and South Asia Meeting, 3.4.69; Kissinger Memo, 29.4.69, FRUS 69-76, E-4, dos. 10; die Ernennung des bisherigen Zentralbankdirektors Mehdi Sami' i (,,perhaps Iran's soundest financial leader“) zum Leiter der Planorganisation weckte neue Hoffnungen auf ein Comeback des pro-amerikanischen Netzwerks in der zentralen Wirtschaftsplanung, doch sollte sich dieser Wunsch nicht erfüllen. Vgl. NIE 34-69 \{Iran\}, 10.1.69, ebd., doc. 1; Tehran \#2100, 4.4.73, FN 1 IRAN, CF 70-73. 
Verdoppelung des Pro-Kopf-Einkommens erreichen: Für das Jahr 1985 waren \$1.000 pro Kopf kalkuliert, in etwa auf dem Niveau einiger europäischer Länder. Voraussetzung war allerdings ein Erfolg der Familienpolitik, um das weiterhin rasante Bevölkerungswachstum - 3,2\% im Jahr 1972 - einzudämmen. ${ }^{211}$ Zugleich versuchte die Regierung, die weiterhin einseitige Abhängigkeit von den Ölressourcen durch Diversifizierung der Wirtschaft zu reduzieren. Der enorme Anstieg des Ölpreises ab 1973 vervierfachte die iranischen Öleinnahmen im Jahr 1974 auf die sagenhafte Summe von \$20 Milliarden, die auszugeben selbst der sonst so ausgabenfreudige Monarch nicht in der Lage war. Neben den exorbitanten Waffenkäufen stiegen auch die Entwicklungsausgaben (und die Rücklagen Irans) rasant an, doch verhinderten diverse „Bottlenecks“, wie die unzureichende Infrastruktur und der Mangel an Fachkräften, zusätzliche positive Effekte in der Gesamtwirtschaft. Im Gegenteil, die Wirtschaft zeigte nun diverse Anzeichen für Überhitzung - im Jahr 1974 stieg die Inflation auf 25\%. Trotz massivem Wirtschaftswachstum erzeugten sich zuspitzende Landflucht und Urbanisierung und die mit dem Bevölkerungswachstum nicht mithaltende landwirtschaftliche Produktion für soziale Unruhe jenseits der hautsächlich profitierenden technokratischen und administrativen Mittelschichten. ${ }^{212}$

Bis 1980 rechnete man mit einer Verdreifachung der amerikanischen Investitionen in Iran bis auf \$2 Milliarden. Ebenso rechnete man infolge des im März 1975 vereinbarten Protokolls im Rahmen der zwei Jahre zuvor eingerichteten U.S.-Iran Joint Commission mit einer Ausweitung des bilateralen Handelsvolumens jenseits des Erdölsektors auf bis zu \$25 Milliarden - eine mehr als willkommene Entlastung der amerikanischen Handelsbilanz. ${ }^{213}$ Die Joint Commission - man wählte das gleiche Vorgehen gegenüber Saudi-Arabien - war mit dem Ziel eingerichtet worden, die Beziehungen zu Iran gerade auf wirtschaftlichem und wissenschaftlichtechnologischem Gebiet zu vertiefen: ,[...] to engage the Iranians so intimately as to assure an enduring relationship under this or successor regimes“, also die zwischengesellschaftlichen, ökonomischen und auch kulturellen Verflechtungen ähnlich $\mathrm{zu}$ den westeuropäischen Verbündeten auszubauen, offenkundig eine Reaktion auf die im Grundsatz veränderte politische Ökonomie der Weltordnung im Nachgang der Ölkrise. Die Verdichtung der gegenseitigen Beziehungen, so hoffte man, sollte zudem verstärkten Einfluss auf die allgemeine Öl- und Ölpreispolitik des Verbündeten und auf das Finanzmanagement der gewaltigen Öleinkünfte ,[...] to the benefit of broader world interests“ eröffnen. Offenkundig spielte auch

\footnotetext{
${ }^{211}$ Vgl. Tehran \#2604, 4.5.72, FRUS 69-76, E-4, doc. 184; „In one generation we will catch up with Europe.“; MemCon, 16.5.75, FRUS 69-76, XXVII, S. 386; Tehran \#A-95, 29.5.73, E 5 IRAN, CF 70-73.

${ }^{212}$ Vgl. NIE 34-1-75 \{,Iran'\}, 9.5.75, FRUS 69-76, XXVII, S. 346-67, hier S. 355ff.; zur Inflation und deren Auswirkungen auf die ärmeren Bevölkerungsschichten vgl. Tehran \#A-166, 17.11.73, E 8-1 IRAN, CF 70-73; CIA/OER Report, 16.10.74, DNSA: IR00899; für die Spannungen in der Arbeiterschaft der Ölindustrie vgl. JAFARI, Iranian Oil Workers; für eine interessante Interpretation der langfristigen Auswirkungen der sozio-ökonomischen Transformationen vgl. FARHI, Class Struggles.

${ }^{213}$ Zum Zeitpunkt des Abschlusses des Protokolls hatte man noch mit einem Volumen von \$12,5 Milliarden gerechnet - innerhalb weniger Wochen ging man bereits von der doppelten Summe aus. Vgl. FRUS 69-76, XXVII, S. 356ff.; vgl. Summary of U.S.-Iranian Protocol Negotiation, o.D. [März 1975], ebd., S. $320 \mathrm{ff}$.
} 
die Hoffnung mit, die jüngeren Belastungen im vormals für die Beziehungen zentralen militärischen Feld durch neue Verknüpfungen im Ökonomischen auszugleichen. ${ }^{214}$ Eine Retrospektive über die bilateralen Beziehungen aus der Post-Revolutionszeit konstatierte allerdings im Nachgang, dass die Joint Commission „[...] ultimately accomplished very little. “215

Ungefähr zeitgleich zur Einrichtung der Joint Commission konstatierte die Defense Intelligence Agency in einer Einschätzung, Mohammad Rezā fühle sich nunmehr angesichts der Hebelwirkung der offenbar endlos sprudelnden Petrodollars nicht mehr zur Rücksichtnahme auf die Interessen der Supermächte verpflichtet. ${ }^{216}$ Die CIA bestätigte diese Sichtweise in ihrem ersten NIE zur Lage in Iran seit sechs Jahren - sie diagnostizierte Mohammad Rezā eine selbstherrliche Attitüde, absolutistische Tendenzen, und einen ansteigenden ,sense of infallibility“. ${ }^{217}$ Bereits zwei Jahre zuvor war eine Einschätzung der Botschaft zu einem ähnlichen Schluss gekommen: Tatsächlich seien Anzeichen von Megalomanie festzustellen. ${ }^{218}$ Militärische Macht und außenpolitische Anerkennung als führender Staatsmann korrespondierten nach innen mit einer zunehmend unreflektierten und die mehrheitliche Stimmung in der Bevölkerung ignorierenden Überhöhung Mohammad Rezās, der PahlawiDynastie und der monarchischen Herrschaftsform in der iranischen Geschichte. Über ein Vierteljahrhundert nach seiner Thronbesteigung hatte sich der Schah im November 1967 endlich zur nachträglichen Krönung entschlossen - mit der Geburt eines männlichen Erbens und der Einrichtung eines Regentschaftsrates waren wichtige Voraussetzungen erfüllt. Die Botschaft in Teheran beschrieb die Ausgestaltung der Krönungswoche als ,lavish in grandeur“, eine Abkehr der zuvor ostentativen Zurückhaltung in der Selbstdarstellung der ohnehin aufgrund der eigenen Herkunft nicht gerade erhaben erscheinenden Dynastie. Triumphbögen und die beschleunigte Fertigstellung einiger symbolischer Bauten und Straßen begleiteten den

\footnotetext{
${ }^{214}$ Interdepartmental Working Group Paper, 25.4.74, FRUS 69-76, XXVII, S. 184; interessant ist zudem die nun Mitte der 1970er Jahre endgültig etablierte Sichtweise, Iran sei in jedem Fall als gleichwertiger Partner zu behandeln. Der früher gängige „faint paternalism“ im Umgang mit Teheran sei zu vermeiden. Ebd., S. 185; zur Anbahnung der Kooperation vgl. auch State \#73527/\#167741, 11.4./1.8.74, ebd., S. 179-82, 221-3; das erste Treffen der Joint Commission fand im November 1974 in Teheran statt. Vgl MemCon, 2.11.74, ebd., S. 259-66; State \#48689, 11.3.74, DNSA: IR00850; zentral war aus US-Sicht offenbar die Einhegung zukünftiger Ölpreiserhöhungen. Kissinger erklärte dem Präsidenten: „Our joint commissions are pushing the producers into big development programs, for which they will need the additional oil production. [...] We may have broken OPEC, or will have if we can make one more deal like this."; MemCon, 4.3.75, ebd., S. $322 \mathrm{f}$.; zu den Hintergründen vgl. auch Hormats Memo, 4.3.75, FRUS 69-76, XXXVIII, S. 159-63.

${ }^{215}$ Department of State, Iran White Paper: Evolution of Relationship, 29.1.80, DNSA: IR03554, S. 21; allerdings nahm man auch im wirtschaftlichen Bereich die bereits im Zusammenhang mit der Schlesinger-Initiative registrierte allgemeine Verschlechterung der vormals exzellenten Beziehungen war. Vgl. Atherton Briefing Memo, 10.11.75, FRUS 69-76, XXVII, S. 439ff.; siehe auch den INR-Report, erwähnt in Auszügen in ebd., S. 439 n3. ${ }^{216}$ Vgl. ebd., S. 329 n3; die DIA sah unter Umständen auch eine außenpolitische Neuorientierung voraus.

${ }^{217}$ NIE 34-1-75: ,Iran', 9.5.75, FRUS 69-76, XXVII,S. 346-67, hier S. 350; der Herrschaftsstil des Schahs sei „,increasingly arbitrary“, er würde auch die Expertise seiner Berater nicht mehr in Anspruch nehmen bzw. habe sich mit ,yes-men“ umgeben. Vgl. Office of Current Intelligence/CIA Paper DCI/NIO 1039-75, ebd., S. 766-81; hier S. 770; innerhalb der CIA wunderte man sich offenbar, dass jahrelang auf eine grundsätzliche geheimdienstliche Einschätzung zu Iran verzichtet worden war. Vgl. Hyland Memo, 5.3.75, CREST. \#RDP80*022-7.

${ }_{218}$ Vgl. Tehran \#A-4, 9.1.73, FRUS 69-76, XXVII, S. 7; die beste Zusammenfassung von Mohammad Rezās Selbstverständnis bietet GRAHAM, Illusion of Power, S. 247; vgl. auch INR Report, ,The Future of Iran: Implications for the US', 28.1.77, DNSA: IR01144.
} 
Anlass. Die Botschaft erwähnte durchaus vernehmbare Kritik an den hohen Kosten - kaum jemand schenkte der offiziell veranschlagten Summe von etwa \$3,5 Millionen Glauben -, sah diese jedoch als vernachlässigbar an angesichts der Freude der Iraner an Pomp und Unterhaltung. Eine für den Anlass von Wiener Handwerkern entworfene vergoldete Kutsche, gezogen von acht Schimmeln, brachte das Herrscherpaar zum Golestanpalast, wo Mohammad Rezā erst sich selbst und anschließend die Šahbānu krönte, letztere mit einer neuen in Paris designten Krone. ${ }^{219}$ Einer der wenigen ausländischen Gäste - auf Wunsch des Schahs wurde die Krönung als durchweg ,lokales“ Ereignis gestaltet -, der ehemalige Under Secretary of State George Ball, beschrieb die Zeremonie als ,,both impressive and depressing, with obtrusive contradictions and pretensions“. 220

An Kosten und Grandezza die Krönung bei weitem übertreffend, erfuhr die 2.500-Jahr-Feier der Monarchie in Iran im Oktober 1971 weltweite Aufmerksamkeit. Die Idee, Feierlichkeiten zum Jubiläum der Gründung des Achämenidenreiches durch Kyros II. abzuhalten, entstand bereits Ende der 1950er Jahre in wissenschaftlichen Kreisen und wurde anfangs nur halbherzig von staatlicher Seite mit Unterstützung bedacht. Die Feierlichkeiten wurden mehrfach verschoben - zum Amüsement amerikanischer Offizieller (,So the astrologers in the Ministry of Court have determined that the Persian Empire was actually born in 529 B.C.!") -, bis Mohammad Rezā schließlich 1970 ein Machtwort sprach und die Feier nun datierte und in einen offiziellen Staatsakt umwandelte. ${ }^{221}$ Die Zeremonien fanden hauptsächlich in der altpersischen Residenzstadt Persepolis statt. Für die Feiern wurde nahe der Ausgrabungen eine - vollständig klimatisierte - Zeltstadt für die internationalen Gäste errichtet - der Pariser Edelausstatter Maison Jansen war für Planung, Bau und Einrichtung verantwortlich. Eigens für das Ereignis wurde nahebei eine Landebahn für zivile Passagierflugzeuge errichtet, das Catering übernahm Maxim's aus Paris: Neben 25.000 Flaschen französischem Wein und Champagner wurden auch über 150 Chefs de Cuisine sowie Kellner aus Europa eingeflogen, um die perfekte Bewirtung

\footnotetext{
${ }^{219}$ Der Titel „Šahbānu“ wurde eigens für diesen Zweck kreiert. Ein iranischer Offizieller sprach gegenüber der Botschaft von 6.-7.000 neuen Einrichtungen, 270 Gedenksäulen und 52.000 Triumphbögen, die zum Anlass der Krönung im gesamten Land errichten worden seien. Auf die Krönung folgten mehrere Tage weiterer Feierlichkeiten mit großen Abendempfängen. Selbstverständlich durfte auch eine Militärparade nicht fehlen, zum ersten Mal die Präsentation sowjetischer Waffensysteme einschließend. Der Schah amnestierte zur Feier des Anlasses 5.000 Gefangene, darunter einige der Attentäter von 1965. Vgl. Tehran \#A-264, 21.11.67, POL 15-1; INR Research Memo RNA-46, 9.10.67, POL 15-1, CF 67-69; Vgl. Hall Memo, 10.12.66, ,EDU 12-4 [...]66', Lot 69 D 491, NEA/IRN: 1964-66, Box 13.

${ }^{220}$ „It was pure factice - a flamboyant attempt to give bogus legitimacy to his parvenu Pahlevi dynasty."; BALL, Another Pattern, S. 435; Bill Miller bezeichnete die Krönung als Wendepunkt hin zu einer Dominanz antiamerikanischer Einstellungen in der Bevölkerung. Siehe William G. Miller OHI, 7.3.85, IOHP, Transcript 2, S. 22.

${ }^{221}$ Zur Vorgeschichte vgl. AfKHAMI, Life and Times, S. 404ff.; in der frühen Planungsphase trug man dem ehemaligen Botschafter Henderson trotz dessen Beteiligung am Sturz Mosaddegs den Vorsitz des „Amerikanischen Komitees“ an, das die Vorbereitungen auf US-Seite koordinieren sollte. Vgl. Minor Memo, 3.1.62, ,2500th Anniversary of Persian Empire 8‘, Lot 69 D 30, NEA/IRN: 1964-66, Box 2; Miklos Memo, 3.1.62, ,Office Memoranda, 1962‘, ebd., Box 4; die Feierlichkeiten wurden zuerst auf den Frühling 1964, daraufhin auf 1965, auf 1967, und schließlich auf 1971 verschoben. Der ehemalige Botschafter Allen unterstützte die Idee, einen kostspieligen Spielfilm über das Leben Kyros’ zu drehen. Vgl. Minor Memo, 6.3.62; Allen Letter, 19.6.62, Miklos Letter, 30.8.62, ,2500th Anniversary of Persian Empire 8‘, Lot 69 D 30, NEA/IRN: 1964-66, Box 2; Tehran \#A20/\#A-288, 12.7./23.11.66, POL 15-1 IRAN, CF 64-66; Tehran \#A-822, 12.9.68, CUL 6 Iran, CF 67-69; Zitat aus Eliot Jr. Letter, 21.11.66, ,EDU 12-4 [...]66“, Lot 69 D 491, NEA/IRN: 1964-66, Box 13.
} 
sicherzustellen. 69 Staaten sandten Vertreter, darunter das sowjetische Staatsoberhaupt Nikolai Podgorny, US-Vizepräsident Spiro Agnew, die jugoslawischen und rumänischen Staatspräsidenten Josip Tito und Nicolae Ceauşescu, die Präsidenten Indiens und Pakistans, der zairische Präsident Mobutu und neben König Hussein von Jordanien und Kaiser Haile Selassie von Äthiopien auch eine ganze Serie von Repräsentanten aus altehrwürdigen und weit weniger erhabenen Dynastien aus Europa, Asien und Afrika. Die Zeremonien in Persepolis und an anderen Orten in Iran - so gab es eine historische Parade mit Tausenden von Statisten - wurden live im amerikanischen Fernsehen übertragen. Die Persepolis-Feiern als extravagant zu bezeichnen, wäre eine Untertreibung; das amerikanische Magazin Newsweek bezeichnete sie trefflich als den „Bash of Bashes“.222

Die 2.500-Jahr-Feier war im historischen Rückblick mehr als ein gesellschaftlicher Höhepunkt für den internationalen Jetset. Tatsächlich ging es Mohammad Rezā um den Anschluss der Pahlawi-Dynastie an die vorislamische Größe Irans, dabei die historisch gewachsene Amalgamierung zoroastrischer und islamischer Vorstellungen und Praktiken von Königsherrschaft zugunsten ersterer neu gewichtend. Die ostentative Rückprojektion auf die achämenidischen (und vorislamischen) Glanzzeiten war dabei mehr als nur der Versuch, die eigene Herrschaft und die grandiosen Ambitionen zu legitimieren und in eine Kontinuität historischer Größe einzuordnen. Zu Beginn der Feierlichkeiten hielt der Schah eine durchaus als albern zu qualifizierende Ansprache am Grabmal Kyros II. in Pasargadae, den verstorbenen Großkönig unmittelbar im Namen der iranischen Nation adressierend, und mit den Worten schließend: „Ruhe in Frieden, denn wir sind wachsam und wir werden immer wachsam bleiben." 223

In eine ähnliche Richtung zielte die zunehmende Grandiosität und der Pomp der Herrschaftsausübung. Dazu zählten die diversen Bestandteile der Titulatur des Schahs: Neben dem etablierten „König der Könige“ oder Großkönig (Šāhanšāh) und dem bereits erwähnten, 1965 beigefügten „Licht der Arier“ (Ärjāmehr) traten eine Reihe weiterer Ehrentitel wie z.B. das auch von den safawidischen Herrschern verwendete „Schatten Gottes auf Erden“ (zill Allāh $f i$ ' $l$-ard $).{ }^{224}$ Die Kontinuität zu den vorislamischen Glanzzeiten wurde in der offiziellen Geschichtspolitik postuliert, die sich wiederum in den Lehrbüchern an Schulen und Universitäten spiegelte. Die Betonung der Rolle der Institution der Monarchie in der iranischen

\footnotetext{
${ }^{222}$ Vgl. CIA Memo, 8.10.71, encl. to Huizenga Memo, 8.10.71, CREST: \#RDP79*014-1; vgl. FARMANFARMAIAN, Blood and Oil, S. 396-400; BILL, Eagle and Lion, S. 183ff.; apologetische Darstellung der Involvierung von Jansen und Maxim's bei AFKHAMI, Life and Times, S. $407 \mathrm{f}$.

${ }^{223}$ Zit. aus BILL, Eagle and Lion, S. 184; ANSARI, Politics of Nationalism, S. 166-79; vgl. GRIGOR, Persepolis '71; in Vorbereitung auf die Zeremonie wurde Pasargadae zuvor von Bauten aus der islamischen Epoche „gereinigt“" Vgl. MOZAFFARI/WESTBROOK, Museum at Pasargadae; Zur Funktion des offiziell-staatlichen Gedenkens vgl. auch LEWIS, History: Remembered, Recovered, Invented.

${ }_{224}$ Zur Anbindung an die achämenidische Vergangenheit vgl. ChOKsy, Ancient Ideas; MERHAVy, Religious Appropriation; angesichts eines üppigen Empfangs wähnte sich ein DDR-Diplomat 1973 ,bei einer Filmaufnahme über das höfische Leben im vorigen Jahrhundert"; Notiz Seidel in Botschaft Teheran an MfAA, 25.8.73, MfAA C $545 / 75$, PAAA.
} 
Geschichte und die Fokussierung auf die ,arische“ Herkunft der Iraner dienten gleichermaßen der Legitimation der eigenen hegemonialen Ansprüche wie der Konstruktion einer Zugehörigkeit der eigenen Zivilisation zum - historisch erfolgreicheren - westlichen Pendant. $^{225}$ Für Iraner unmissverständlich war dabei die Zielrichtung gegen die Rolle der Religion und die Geistlichkeit in der eigenen Geschichte. Die Zeit arabischer Herrschaft - und damit der Ankunft des Islam - wurde als Periode der Barbarei verunglimpft. Zugleich wurden Anstalten unternommen, den Einfluss arabischer Kultur zurückzudrängen. So versuchte man, das Neupersische von arabischen und türkischen Lehnwörtern zu „säubern“. 226 Noch eindeutiger anti-islamisch in der Ausrichtung war die von Mohammad Rezā 1975 verfügte Kalenderreform, in Folge derer der von seinem Vater eingeführte Sonnenkalender durch einen neuen ersetzt wurde, wobei die Hidschra des Propheten als Anfangspunkt der Zeitrechnung nunmehr ersetzt wurde durch das Jahr der Krönung Kyros II im Jahr 559 v. Chr. Statt im Jahr 1355 datierte man nun das Jahr 2535. Wenig überraschend erließ Khomeini umgehend eine Fatwa, die den Gläubigen den Gebrauch des imperialen Kalenders untersagte. ${ }^{227}$

Analog spiegelte sich die angestrebte Grandiosität in den öffentlichen Bauvorhaben wieder. Im Westen Teherans entstanden im Kontext der 2.500-Jahr-Feierlichkeiten das riesige ĀrjāmehrStadion und das rasch zum optischen Symbol der Hauptstadt (und später der Islamischen Revolution) avancierende Monument des borğ-e šahjād (heute Freiheitsturm/borğ-e āza adi) ${ }^{228}$ Der neue modernistische Baustil verdrängte die neo-achämenidische und neo-sassanidische Architektur. Wenngleich widersprüchlich, so ging doch damit eine Kehrtwende hin zu mehr Opulenz einher. Während sich das Mausoleum des Dynastiegründers Rezā Xān in Ray, 1951 erbaut, bescheiden gab, waren Mohammad Rezās Bauvorhaben auf maximale Repräsentation, Glanz und Gepränge ausgerichtet. ${ }^{229}$ Offenbar gab es bereits Planungen für den Bau einer monumentalen Begräbnisstätte für Mohammad Rezā - 114 Architekten, in der Mehrzahl Ausländer, waren an dem Projekt beteiligt. ${ }^{230} 1975$ gab der Monarch die Konstruktion eines neuen urbanen Zentrums mit gewaltigen Ausmaßen für die Hauptstadt Teheran - Šahestan Pahlawi genannt - in Auftrag. ${ }^{231}$

Die Instrumentalisierung der iranischen Vergangenheit war Teil einer, wenngleich inkohärenten und teils widersprüchlichen Herrschaftsideologie des „Pahlawismus“, die sich

\footnotetext{
${ }^{225}$ Vgl. SHAKIBI, Pahlavīsm.

${ }^{226}$ Zur Vorgeschichte im Kontext des iranischen Nationalismus vgl. KIA, Language Purification.

227 Vgl. Buchan, Days of God, S. 193; AfKhami, Life and Times, S. 410f.; CHOKsy, Ancient Ideas, S. 57f.; GrAHAM, Illusion of Power, S.61; ZoNIS, Majestic Failure, S. 81.

${ }^{228}$ Das $\breve{S} a h j \bar{a} d$-Denkmal sollte die inzwischen auf 12 angewachsenen Hauptprinzipien der Weißen Revolution symbolisieren. Vgl. GRIGOR, Revivalism, S. 245f.; die Stätten wurden nach der Revolution in Freiheits-Denkmal und -Stadium umbenannt.

${ }_{229} \mathrm{Zu} \mathrm{Rezā} \mathrm{Šāhs} \mathrm{Mausoleum} \mathrm{als} \mathrm{ostentativer} \mathrm{Abkehr} \mathrm{von} \mathrm{der} \mathrm{Opulenz} \mathrm{der} \mathrm{Ġāğāren} \mathrm{vgl.} \mathrm{ebd.,} \mathrm{S.} \mathrm{239;} \mathrm{das}$ Mausoleum wurde 1980 in der Revolution durch einen von Ājatollāh Xalxāli angeführten Mob eingeebnet. Vgl. BUCHAN, Days of God, S. 324.

${ }^{230}$ Vgl. MemCon, 31.8.77, Asnād 7, S. 227.

${ }^{231}$ Zur Zeit des Ausbruchs der Revolution stand die Umsetzung noch in ihren Anfängen. Vgl. Emami, New Urban Centre; vgl. MASHAYEKHI, Scene of Modernity.
} 
seit der zweiten Hälfte der 1960er Jahre schrittweise etabliert hatte. ${ }^{232}$ Inkohärent musste die Ideologie zwangsläufig sein, suchte sie doch die nahezu absolute Herrschaft Mohammad Rezās als einerseits in der Kontinuität der iranischen Monarchie stehend und auf Gottesgnadentum begründet darzustellen, andererseits aber auch „modern“ legitimierend, unter Verweis auf die erfolgreiche und segensreiche Transformation der iranischen Gesellschaft mittels einer „Revolution von oben“, durchaus im Einklang mit westlichen Modernisierungs- und Fortschrittsvorstellungen. Attraktiv aus Sicht des Herrscherhauses war dabei die Möglichkeit, iranischen Nationalismus mit monarchischen Absolutismus $\mathrm{zu}$ verbinden, gleichzeitig Demokratie und Konstitutionalismus hintanstellend. An die Stelle westlicher Vorstellungen gesellschaftlicher und politischer Organisation trat die Idee einer überlegenen und spezifisch iranischen Ordnung, basierend auf einem patronisierenden Verhältnis zwischen Monarchen und Volk, zugleich einen Dritten Weg zwischen kapitalistischen und kommunistischen Alternativen aufzeigend, die „Große Zivilisation“ (tamaddon-e bozorg). ${ }^{233}$

Das antikisierende Element, der Rückgriff auf die glorreiche präislamische Zeit, findet sich bereits in der Eigendarstellung bei Rezā Šāh. Bei dessen Sohn aber scheint gerade die Außenwirkung, die Wahrnehmung der Bezugnahme des angedachten neuen persischen Großreiches auf seine beeindruckenden historischen Vorbilder durch ausländische Beobachter, eine mindestens ebenso große Rolle gespielt zu haben. Anders als noch bei den Krönungsfeierlichkeiten von 1967 standen bei den 2.500-Jahr-Feiern die ausländischen Würdenträger und insbesondere die Steuerung der Berichterstattung durch die internationalen Medien im Mittelpunkt. Ein aufwändiger Dokumentarfilm rund um die Feierlichkeiten mit Orson Welles als Sprecher wurde für ein westliches Publikum produziert. ${ }^{234}$ Trotz aller Anstrengungen wurde der Extravaganz in der westlichen Öffentlichkeit nur wenig Aufmerksamkeit zuteil, und wenn überhaupt, dann zumeist eine recht kritische. Fataler aber war der innenpolitische Effekt. Insbesondere die Frage des tatsächlichen finanziellen Aufwandes erhitzte die Gemüter, eine Debatte, die sich bis zum heutigen Tag fortsetzt. Hofminister Asadollah 'Alam, hauptverantwortlich für die Organisation, bezifferte die Kosten im Nachgang mit etwa \$17 Millionen, was aber offenbar nur die unmittelbaren Kosten der Feierlichkeiten selbst umfasste und angesichts der begleitenden Bauvorhaben und des Einsatzes von 50.-100.000 Statisten allein für die Parade zu tief angesetzt sein dürfte. Die sich in späteren akademischen Beurteilungen findenden Schätzungen von \$200-300 Millionen scheinen aber

\footnotetext{
${ }^{232}$ Für den Versuch von offizieller Seite, ein ideologisches Gesamtkonstrukt des „Pahlawismus“ zu konstruieren vgl. SHAKIBI, Pahlavīsm; vgl. auch ADIB-MoghadDAM, Global Grandeur; BAYNE, Persian Kingship, S. 94-43; auch die zeitgenössische CIA sprach vom „Pahlawismus“. Vgl. CIA Intel Report 2035-72 \{,Centers of Power in Iran'\}, Mai 1972, FRUS 69-76, E-4, doc. 180.

${ }^{233}$ Vgl. ShaKIBI, Pahlavīsm, S. 130-3; CHOKsy, Ancient Ideas, S. 52ff.; AzIMI, Quest for Democracy, S.290f.; SchAYEgh, Civilisational Developmentalism; Anleihen an Lyndon Johnsons umfassendes sozialpolitisches Reformprogramm der Great Society sind wahrscheinlich. Zum Einsatz des neuen Ölreichtums zugunsten erster Maßnahmen in Richtung eines Wohlfahrtsstaates vgl. Tehran \#A-28, 20.2.75, DNSA: IR00936.

${ }^{234}$ Das internationale Publikum als Zielobjekt der 2.500-Jahr-Feiern betont auch die Neubewertung von STEELE, Persepolis Celebrations; zu „Flames of Persia“ vgl. WATson, Shah's Quest.
} 
übertrieben - realistisch dürften die Kosten irgendwo zwischen \$50-100 Millionen anzusiedeln sein. ${ }^{235}$ Ungeachtet der tatsächlichen Kosten war die allgemein negative Rezeption seitens der iranischen Bevölkerung augenfällig. Die US-Botschaft beschrieb die private Kritik am „fandance in Shiraz“ seitens iranischer Bürger als ,expensive, unnecessary and tasteless. “236

Die Eigendarstellung der Pahlawi-Dynastie hatte sich zu Beginn der 1970er Jahre gewandelt, mit Tendenzen hin zum Personenkult, Grandiosität in der Repräsentation, und zunehmend totalitären Zügen in der Innenpolitik. Die CIA konstatierte in einer Analyse der internen Machtstrukturen Irans und der Rolle des Schahs vom Mai 1972:

A non-charismatic leader, he has taken on many of the trappings of totalitarianism; scarcely a town of any size does not have its Avenue Pahlavi and it is a mean city, indeed, that does not have a traffic circle dominated by a statue of the Shah or his father. Massive rallies are held, complete with giant portraits of the Shah and banners bearing quotations by him, and no politician ventures a suggestion without carefully pointing out that it fits within the framework approved by the Shah. In fairness, it is to be noted that Iranian monarchs have always surrounded themselves with symbols of their power and the bulk of the population expects them to..$^{237}$

Anfang der 1970er Jahre mehrten sich die Anzeichen, dass sich die seit Mitte des vorhergehenden Jahrzehnts von amerikanischer Seite wiederholt konstatierte innenpolitische Ruhephase dem Ende zuneigte. 1967 hatte man noch seitens des State Department explizit festgestellt, die ,[...] internal Iranian political scene has not been as stable as it is today since World War II.“ Das Reformprogramm Mohammad Rezās, so die Analyse, habe die allermeisten Iraner zu einer Zusammenarbeit mit der politischen Führung bewogen und eine neu entstandene Mittelschicht, Hauptprofiteur der jüngsten Reformen, habe ein eigennütziges Interesse am Erhalt der bestehenden Ordnung entwickelt. ${ }^{238}$ Die CIA zeigte sich zwei Jahre später allerdings skeptischer, was den Erfolg der politischen Integration der ehemals opponierenden nationalistischen Intellektuellen anging: Zwar habe die Schaffung technokratischer Karrieren viele ehemalige Oppositionelle zum Eintritt in den Staatsdienst und zur Fokussierung auf ,,moneymaking“ bewegt, doch sei die zur Schau gestellte Loyalität nur oberflächlich und im Krisenfall mit einer raschen Desertion der neuen Mittelschichten zu

\footnotetext{
${ }^{235}$ Vgl. die apologetische Darstellung bei AfKhami, Life and Times, S. 412-15; 2-300 Millionen bei Bill, Eagle and Lion, S. 183f., 482 n2; SHAwCross, Shah's Last Ride, S. 47; vgl. BuCHAN, Days of God, S. 54-7; \$30-100 Millionen bei FARMANFARMIAN, S. 396-400.

${ }_{236}$ Escudero Letter, 18.12.72, ,POL 1-Gen[...]72‘, Lot 75 D 351, NEA/IRN: 1965-75, Box 7; die Feiern entschieden auch einen der internen Machtkämpfe - Ašraf, die Zwillingsschwester des Schahs, erreichte nun die Entlassung ihres Intimfeindes Ardešir Zāhedi als Außenminister, der für die als unangemessen empfundene Repräsentation ausländischer Staatsoberhäupter verantwortlich gemacht wurde. Vgl. CIA Intel Report 2035-72, Mai 1972, FRUS 69-76, E-4, doc. 180; zur US-Repräsentation bei den Feierlichkeiten vgl. Kissinger Memo, o.D., ,Iran, Vol. III, 1 Jan-31 Aug 71', NSCF, Country Files-Middle East, Box 602, RMNL.

${ }_{237}$ CIA: CIA Intel Report 2035-72, Mai 1972, FRUS 69-76, E-4, doc. 180.

${ }^{238}$ State Background Paper, 15.8.67, FRUS 64-68, XXII, S. 405; die Opposition sei de facto zerschlagen: Die Nationale Front sei ,destroyed in all but name by government suppression and internal dissension." Die „Freiheitsbewegung Irans“ (Nehzat-e Äzādi-je Irān) und die obskure „Partei der Islamischen Nationen“ (hezb-e melal-e eslämi) seien staatlichen Verfolgungen ausgesetzt. Zudem habe der nationalistische Kurs des Schahs nach innen wie außen den vormaligen Monopolanspruch gerade der Nationalen Front auf die Gefolgschaft iranischer Nationalisten unterminiert - dem Schah sei gelungen, das Stigma der Servilität gegenüber dem Westen abzulegen. Vgl. Hughes Memo [INR Research Memo RNA-12], 27.3.68, POL 15-1 IRAN, CF 67-69.
} 
rechnen: „Over the long term, economic development probably will not provide a satisfactory substitute for greater political participation.“239 Die noch vom institutionellen Gedächtnis der Krisenjahre zwischen 1958 und 1963 geprägte Skepsis war wenige Jahre später angesichts der atemberaubenden wirtschaftlichen und außenpolitischen Erfolge des Schahs weitgehend verflogen. Die Botschaft sagte eine unangefochtene Dominanz Mohammad Rezās im politischen System voraus, der wie ein „Colossus“ die politische Szene überrage. ${ }^{240}$ Allerdings müsse auf Dauer die politische Basis des Regimes verbreitert werden.

Gerade gegenüber westlichen Gästen kokettierte der Schah gerne mit seiner zukünftigen Absicht, den erfolgreichen wirtschaftlichen Reformen auch bald politische folgen lassen zu wollen. ${ }^{241}$ Die mehrfach angekündigten, freieren Kommunalwahlen wurden nicht abgehalten. Die als Staatspartei und Sammelbecken gedachte Partei des Neuen Iran (Hezb-e Irān-e nowin) war bald nicht mehr als ein verlängerter Arm der Staatsbürokratie. Die anfangs postulierte breitere politische Partizipation wurde nicht einmal ansatzweise umgesetzt: „The Shah seems to have made a conscious decision to emphasize the pursuit of higher standards of living in order to keep Iranian minds off any movement to secure participation in the political process [...]“, so INR im Jahr 1968. ${ }^{242} 1973$ sah die Botschaft den Schah zumindest intellektuell demokratischen Ideen zugeneigt, doch fürchte er eine Destabilisierung des Landes selbst bei Gewährung geringer Partizipation, gerade aufgrund der Erfahrungen der Mosaddeg-Zeit. ${ }^{243}$ Die Intelligence Community widersprach zwei Jahre später resolut. Der Schah beabsichtige keineswegs, in irgendeiner Form die Macht zu teilen. ${ }^{244}$ Im Gegenteil, Mitte der 1970er Jahre entschied sich Mohammad Rezā auch die bestehende demokratische Fassade zu beseitigen der Pseudo-Wettbewerb zwischen der Regierungspartei Hezb-e Irān-e nowin und der ,loyalen Opposition“ der Mardom-Partei wurde aufgehoben und im März 1975 die neue Einheitspartei, die Hezb-e Rastāxiz (die Partei des „Wiederaufstiegs“) aus der Taufe gehoben. Die CIA konstatierte lapidar, der Schah ,lost interest in the facade of competitive politics." ${ }^{245}$ Alle bestehenden Parteien, auch die beiden bislang tolerierten ultranationalistischen paniranistischen Parteien gingen in die Rastāxiz-Partei auf. Allein die Tatsache, dass der langjährige Premier Howejdā zum Generalsekretär der neuen Schöpfung berufen wurde,

\footnotetext{
${ }^{239}$ NIE 34-69 \{,Iran'\}, 10.1.69, FRUS 69-76, E-4, doc. 1.

240 „His total success has enhanced his prestige and underlined his multifaceted position as stern ruler, national guide and mentor, remote but omniscient father-figure and, to some, reactionary oppressor and destroyer of individual liberties. The imperial influence, real or imagined, now extends to virtually all levels of Iranian society."; Tehran \#A-4, 9.1.73, FRUS 69-76, XXVII, S. 2; die CIA berichtete 1972, in der Zwischenzeit sei auch der zuvor einflussreiche Hof entmachtet - allein das Wort des Schahs zähle. Vgl. CIA Intel Report 2035-72 $\{$,Centers of Power in Iran'\}, Mai 1972, FRUS 69-76, E-4, doc. 180.

${ }^{241}$ Vgl. z.B. Tehran \#2410, 5.12.67, FRUS 64-68, XXII, S. 450.

${ }^{242}$ Vgl. Hughes Memo [INR RNA-12], 27.3.68, POL 15-1 IRAN, CF 67-69; Teile in FRUS 64-68, XXII, S. $491 \mathrm{f}$. ${ }^{243}$ Vgl. Tehran \#A-4, 9.1.73, FRUS 69-76, XXVII, S. 1-19.

244 ,His willingness to tolerate limited party competition stemmed from the desire to appease critics in the West and among Western-influenced elements of the population. But he has long been mistrustful of the parliamentary process.“; NIE 34-1-75 ,Iran“, 9.5.75, ebd., S. 346-67, hier 349; vgl. auch CIA Memo, 12.8.77, DNSA: IR01213. 245 NIE 34-1-75, vorherig. Anm., S. 351; mit ein Grund für die Aufhebung der pseudo-demokratischen Parteienvielfalt war offenbar die unerwünschte basisdemokratische Debatte in der Mardom-Partei gegen die Regierungspolitik einige Jahre zuvor. Vgl. Killgore Letter, 30.10.72, FRUS 69-76, E-4, doc. 230.
} 
demonstrierte jedoch, dass an den bestehenden Verhältnissen kaum gerüttelt werden sollte. Von allen wahlberechtigten Iranern wurde der Eintritt in die Rastāxiz-Partei erwartet allerdings reagierte die Masse der Bevölkerung auf diese Neuerung mit der von allen Beobachtern erwarteten Apathie. ${ }^{246}$ Ein Manifest Khomeinis aus dem Exil, das Mitgliedschaft in der neuen Partei als Bruch religiöser Vorschriften brandmarkte, erhielt offenbar weite Verbreitung in Iran. ${ }^{247}$

Die graduelle Aufhebung des für Iran so charakteristischen „,gesteuerten Pluralismus“ seit Mitte der 1960er Jahre wurde begleitet von der zunehmenden Repression gerade durch den immer mächtiger werdenden SAWAK, gleichermaßen in Reaktion auf das Erscheinen der urbanen Guerillas und als Ausdruck des sich ausweitenden Machtverständnisses Mohammad Rezās, der immer weniger bereit war, interne Kritik zu tolerieren. Die Anfang des Kapitels geschilderten Unmutsbekundungen während der Asienmeisterschaft 1968 waren ein Anzeichen für die sich entwickelnde Oppositionsbewegung. Vorreiter war einmal wieder die Studentenschaft. 1968 noch hatte Botschafter Meyer das Problem des politischen Aktivismus an iranischen Universitäten als beherrschbar eingeschätzt. ${ }^{248} 1970$ weiteten sich die Proteste allerdings aus, als die Studentenschaft begann, Themen jenseits der Bildungspolitik, insbesondere soziale Fragen, aufzugreifen. Eine Erhöhung der Busfahrpreise musste nach dreitägigen Zusammenstößen zwischen Studenten und Polizei in Teheran zurückgenommen werden. Neuerliche Proteste gegen die 2.500-Jahrfeier an der Universität Teheran und an der ĀrjāmehrUniversität, ebenfalls in der Hauptstadt, im Frühling 1971 führten zu einer gewaltsamen Reaktion der Sicherheitskräfte - und in Reaktion darauf zu einer Massendemission fast der gesamten Fakultät der Ārjāmehr. In der Botschaft wurde angesichts der Unruhe unter der Jugend ein Embassy Youth Committee eingerichtet, das die Einstellung der jüngeren Generationen und ihre Auswirkungen auf das bilaterale Verhältnis untersuchen sollte. ${ }^{249}$

Besonders beunruhigend aus amerikanischer Perspektive waren die nicht unbeträchtlichen

\footnotetext{
${ }^{246}$ Vgl. Tehran \#2069, 4.3.75, FRUS 69-76, XXVII, S. 327ff.; vgl. BILL, Eagle and Lion, S. 221ff.; AFKHAMI, Life and Times, S. 423-40; Hintergründe eher obskur bei AMINI, Single Party State, S. 132f., 162f.

${ }^{247}$ Vgl. CIA Intel Information Cable, 8.5.75, FRUS 69-76, XXVII, S. 344ff.; für eine provokative Interpretation der Rastāxiz-Partei als Einleitung einer anti-westlichen Spielart des Pahlawismus SHAKIBI, Rastakhiz Party.

${ }^{248}$ Vgl. Tehran \#5600, 7.7.68, FRUS 64-68, XXII, S. 537ff.; Tehran \#A-476, 9.3.68, POL 13-2 IRAN, CF 67-69; auch die CIA bewertete in ihrer Studie über den globalen Aktivismus der Studentenschaft diese Phase an iranischen Universitäten als vergleichsweise ruhig, zumindest gemessen an den Jahren vor 1963. Vgl. CIA Study No. 0532/70, August 1970, encl. to Helms Memo, 2.9.70, FRUS 69-76, E-4, doc. 84; die Unruhen in iranischen Universitäten in den Jahren 1967 und 1968 waren allerdings durchaus gravierend. Erst mit den Unruhen von 1971, die an Zahl und Radikalität der Forderungen die Vorjahre übertrafen, reagierte die Botschaft alarmiert. Vgl. Tehran \#A-136, 10.5.71, ebd., doc. 126; Das Youth Committee konstatierte ein zwiespältiges und sich graduell ins Negative kehrendes Image der USA gerade bei der jungen Generation. Siehe Tehran \#A-56, 22.2.71, ebd., doc. 116; ein US-Offizieller kommentierte, dass man der neuen Generation nur näher kommen könne, indem man ,,[...] do that would be for the United States to urge, and be seen to be urging, further steps towards liberalization upon the Shah. For a variety of reasons I am sure we would be most hesitant to do this. Therefore I conclude that we should not worry too much about the ill-informed views of activist youths. By the time they reach positions of influence it should be apparent to them that the United States has a lot to offer Iran."; ebd., $n 1$; vgl. SHANNON, Losing Hearts and Minds, S. 227.

${ }^{249}$ Vgl. ebd., S. 231-6; CIA Report, ,Restless Youth', September 1968, ,CIA Report «Restless Youth», 9/68‘, NSF, Intelligence File, Box 3, LBJL; vgl. Tehran \#668, 24.2.70, FRUS 69-76, E-4, doc. 49.
} 
Sympathien unter den Studenten, aber auch in der übrigen Bevölkerung, für die Aktionen der Guerilla-Organisationen der „Volks-Fedajin“ und der „Volksmudschahedin“, die im Februar 1971 mit einem Angriff der ersteren auf eine Polizeistation in Sijāhkal bewaffnete Aktionen gegen das Regime aufgenommen hatten. Insbesondere die scharfen Gegenmaßnahmen der Regierung - die 13 Angreifer vom Februar wurden gefasst und trotz internationaler Gnadengesuche hingerichtet - heizten die Atmosphäre an. ${ }^{250}$ In den Folgejahren kam es zu etwa 400 Anschlägen gegen staatliche Institutionen und Offizielle - Ervand Abrahamian ermittelte später die Zahl von 368 jungen Guerillas, die zwischen 1971 und 1977 im Kampf gegen die Obrigkeit den Tod fanden. ${ }^{251}$ Im Anschluss an eine Serie von Bombenanschlägen während Nixons und Kissingers Iranbesuch im Mai 1972 bestätigte INR die Existenz eines authentischen gewaltbereiten „Youth Underground“ in dem Land, vorwiegend bestehend aus entfremdeten jungen Erwachsenen mit meist kommunistisch inspirierter Weltanschauung. ${ }^{252}$ Abgesehen von einem erfolgreichen Attentat der Guerilla-Bewegungen auf den Schah schätzte man aber seitens der USA den Terrorismus in Iran nicht als strategische Bedrohung ein.

Mehr Sorgen bereiteten die langfristigen Folgen für die Stabilität des Status Quo, die sich aus dem Ausbau der Kapazitäten und der Handlungsfreiheit des SAWAK ergaben. Die Organisation wuchs unter dem Direktor General Ne'mat-ollāh Nassiri auf über 5.000 Mitarbeiter und eine große Zahl von Informanten an. Anders als noch in den 1960er Jahren reagierte aber nun eine kritische internationale Öffentlichkeit auf die zunehmende Repression in Iran, angeregt durch die immer mehr Aufmerksamkeit erfahrenden Kampagnen der oppositionellen iranischen Studentenschaft im Ausland. Der Amnesty InternationalGeneralsekretär ging 1975 so weit, öffentlich zu erklären, dass ,[...] no country in the world has a worse record in human rights than Iran." ${ }^{253}$ In der Botschaft mehrten sich die Berichte, wonach der Anstieg der Gewalt und die Rekrutierungserfolge der Terrororganisationen auf die Willkür des SAWAK zurückzuführen seien. In den Folgejahren kam es wiederholt zu großen Protestaktionen an den Universitäten, immer gefolgt von repressiven Gegenmaßnahmen des Staates. $^{254}$ Die Anzahl politischer Gefangener in Iran stieg von einigen Hundert Mitte der 1960er Jahre auf mehrere Tausend an - Amnesty International reproduzierte Mitte der 1970er Jahre die übertriebenen Berichte von Exil-Iranern, denen zufolge es bis zu 100.000 politische Gefangene in Iran gäbe. Nach einem Besuch des Generalsekretärs der Organisation auf

\footnotetext{
${ }^{250} \mathrm{Zu}$ den Sympathien vgl. Situation Report [CIA?], 28.2.72; Tehran \#1381, 9.3.72, ebd., docs. 168, 174; Botschafter Douglas MacArthur II behauptete anfangs, die verschiedenen Operationen seien auf Subversionsmaßnahmen von außen zurückzuführen, ein weiterer Beleg für die zunehmende Rolle des SAWAK für die US-Informationsbeschaffung über interne Verhältnisse in Iran. Siehe Tehran \#2495, 12.5.71, ebd., doc. 127.

${ }^{251}$ Vgl. BiLl, Eagle and Lion, S. 483 n14; ABrahamian, Tortured Confessions, S. 101-8; vgl. VAHABZADEH, Guerrilla Odyssey, S.257ff.

${ }^{252}$ Vgl. INR Intel Note RNAN-18 \{,Iran: Internal Dissidence - A Note of Warning '\}, 12.6.72, FRUS 69-76, E-4, doc. 203; siehe auch die besorgte Meldung der US-Botschaft über die Anschlagserie in Tehran \#4789, 10.8.72, ebd., doc. 217; vgl. Tehran \#2495, 12.5.71, POL 23 IRAN, CF 70-73; vgl. COOPER, Oil Kings, S. 61ff.

${ }^{253}$ Vgl. SHANNON, Losing Hearts and Minds, S. 243, 269-79.

${ }^{254}$ Vgl. Tehran \#A-151, 9.9.72, FRUS 69-76, E-4, doc. 220; Tehran \#8619, 6.12.73, FRUS 69-76, XXVII, S. 160ff.; ebd., S. 162 n3.
} 
Einladung des Schahs Anfang 1977 nannte man im nächsten Jahresbericht die Zahl 3.200, die „offizielle“ Angabe des SAWAK, sprach aber weiterhin von einer unbekannten Dunkelziffer. Eine US-Studie zum Terrorismus-Phänomen sprach von allein 2.158 verhafteten Terroristen zwischen März 1974 und 1975, einem Anstieg von 50\% zum Vorjahr, was die in neuerer Literatur zu findenden Schätzungen einiger Tausend politischer Gefangener realistisch erscheinen lässt. ${ }^{255}$ Das nach amerikanischem Vorbild erbaute und 1971 fertiggestellte, berüchtigte Evin-Gefängnis symbolisierte alsbald die Menschenrechtslage in Iran unter dem Schah. Kontraproduktiv waren auch die häufig durch Folter und Drohungen erzwungenen öffentlichen Schuldeingeständnisse einiger inhaftierter Intellektueller, die die Verbitterung über die staatliche Repression meist nur verstärkten. Zugleich mehrten sich glaubwürdige Berichte über eine Ausweitung der Folter in den Gefängnissen und über politische Morde, ausgeführt seitens des SAWAK. So ermordete der SAWAK - im Auftrag oder zumindest mit Billigung des Schahs - im April 1975 neun Vertreter der radikalen Linken, darunter den bekannten Intellektuellen Bižan Ğazani, außerhalb der Mauern des Evin. ${ }^{256}$ Trotz der zunehmenden Repression in Iran entschloss sich die US-Botschaft unter Richard Helms 1976 in einem langen Telegramm, die Vorwürfe zu entkräften bzw. zu bagatellisieren, wenngleich man in Washington durchaus kritisch anmerkte, die meisten Informationen der Botschaft stammten selbst vom SAWAK und dürften daher kaum als objektiv betrachtet werden. ${ }^{257}$ Seitens INR merkte man kritisch an, der Bericht ,should be read with caution as some of its assertions contradict even unclassified State Department reportage and its analysis of torture and press control runs counter to a wealth of classified material [...]. “258

Die zunehmende Repression wurde dennoch immer mehr zu einer zusätzlichen Belastung der bilateralen Beziehungen zwischen den USA und Iran. Ein weiterhin bestehender Streitpunkt zwischen beiden Ländern war die aus iranischer Sicht unzureichende Zusammenarbeit der amerikanischen Behörden gegen oppositionell eingestellte iranische Studenten. Im Kontext der Verschärfung staatlicher Repression hatte der Schah im Januar 1971 die Mitgliedschaft in der internationalen Dachorganisation iranischer Auslandsstudenten, der Confederation of Iranian Students National Union (CISNU), unter Strafe gestellt. ${ }^{259}$ Wenige Monate später präsentierte

\footnotetext{
${ }^{255}$ Vgl. BuChan, Days of God, S. 182ff.; vgl. INR Report No. 146, 7.10.75, FRUS 69-76 XXVII, S. 436-9; Abrahamian, Tortured Confessions, S. 108, schätzt die Gesamtzahl politischer Gefangener Mitte der 1970er Jahre auf 7.500 Personen. Die überzogenen Zahlen halten sich in der Forschung trotzdem bis heute. Siehe beispielsweise SCHAYEGH, Like a State, S. 43; einen Überblick zur Geschichte der Repression im Iran im 20. Jahrhundert bietet MATIN-ASGARI, Iran's Political Prisoners.

${ }^{256} \mathrm{Zu}$ den ,recantations“" vgl. ABRAHAMIAN, Tortured Confessions, S. 112-9; BuCHAN, Days of God, S. $186 \mathrm{f}$.

${ }^{257}$ Vgl. Tehran \#8376, 18.8.76, FRUS 69-76, XXVII, 551-7; vgl. ebd., S. 552f. $n 6$.

${ }^{258}$ Huddle Jr. Memo, 20.8.76, ebd., S. 558; das Memo erwähnt unter anderem die politischen Morde seitens des SAWAK.

${ }^{259}$ Die auf Nordamerika beschränkte Iranian Student Association in the United States hatte sich $1962 \mathrm{mit}$ ihrem europäischen Pendant zur CISNU zusammengeschlossen. Anfangs dominiert von studentischen Parteigängern der Nationalen Front, setzten sich ab Mitte der 1960er Jahre linke und marxistische Kräfte durch, am Ende der Dekade dann maoistische Gruppierungen. Vgl., SHANNON, Losing Hearts and Minds, S. 90, 210-46, 254ff.; wieder einmal drängte das State Department auf Kooperation mit den iranischen Sicherheitsbehörden hinsichtlich der Vergabe amerikanischer Visa. Vgl. Miklos Memo, 24.1.72, ,Iran 1972‘, Lot 73 D 376, NEA/Deputy Assistant Secretary, Rodger P. Davies Subject Files, Box 2.
} 
der SAWAK das öffentliche Geständnis eines angeblichen Überläufers aus den Reihen der linken Studenten - der junge Student behauptete Anwerbeversuche seitens amerikanischer Dienste während seiner Studienzeit in den USA und zudem eine enge Kooperation zwischen CISNU und dem FBI, um den Schah und die kaiserlichen Familienmitglieder auf ihren Auslandsreisen zu diskreditieren. Tatsächlich hatte der Schah bereits in Interviews auf eine geheimdienstliche Steuerung der studentischen Opposition seitens der USA und Großbritanniens verwiesen, wohl gleichermaßen als Gegennarrativ gegen sein Image als prowestliche Marionette gedacht, aber auch als authentischer Ausdruck seiner durchaus als paranoid zu bezeichnenden Wahrnehmung zu interpretieren, die er in ähnlicher Weise auch nach der Revolution bekräftigen sollte. ${ }^{260}$ Im Kontext der größeren Aufmerksamkeit für iranische Menschenrechtsverletzungen häuften sich zudem ab Mitte der 1970er Jahre Berichte in US-Medien über Operationen des SAWAK gegen Oppositionelle im Ausland. Die FordAdministration warnte die iranische Regierung wiederholt vor Gesetzesüberschreitungen durch SAWAK-Agenten auf amerikanischem Staatsgebiet und insbesondere seitens des Stationsleiters des iranischen Geheimdienstes in Washington, Mansur Rafi zādeh. Helms dagegen sprach sich von Teheran gegen jede Form von öffentlichen Schuldzuweisungen „over possibly ill-advised intelligence activity“ aus und erinnerte warnend an die erheblichen Abhängigkeiten der USA in Bezug auf geheimdienstliche Kooperation mit Iran: ,[...] we are very beholden here in the intelligence area and therefore correspondingly vulnerable. “261 Der Konflikt um die SAWAK-Operationen schwelte aber weiter und führte alsbald zu einer Reihe von kritischen Reportagen in US-Medien und Anhörungen im Kongress. ${ }^{262}$

Mittelfristig ähnlich erfolglos wie verdeckte SAWAK-Operation im Ausland waren die propagandistischen Maßnahmen des Pahlawi-Regimes gegen die Kritik von außen an Repression und Menschenrechtsverletzungen in Iran. Irans Gastgeberrolle für die erste internationale UNO-Konferenz über Menschenrechte im April 1968 - eine durchaus als bizarr einzuschätzende Wahl des Veranstaltungsortes angesichts der Thematik - erzeugte im Ausland

\footnotetext{
${ }^{260}$ Die US-Botschaft protestierte gegen die Veröffentlichung des „Geständnisses“ - die Iraner versprachen, weitere Berichterstattung zu vermeiden, doch signalisierten sie zugleich ihre Unzufriedenheit mit der aus ihrer Sicht inakzeptablen Nachsicht gegenüber den oppositionellen Studenten in den USA. Vgl. Tehran \#3128/\#3146/\#3242, 14./14./17.6.71, FRUS 69-76, E-4, docs. 130, 131, 132. Vgl. SHANNON, Losing Hearts and Minds, S. $256 \mathrm{f}$. ${ }^{261}$ Vgl. Tehran \#10874/\#1, 1.11.76/3.1.77; State \#313328, 30.12.76, FRUS 69-76, XXVII, S. 574f., 594f., 591f.; Helms-Zitat in ebd., S. 591 n2; TelCon, 8.11.76, DNSA:HAKTelCons: \#KA15390; Rafi'zādeh verfasste später Memoiren über seine Zeit als SAWAK-Chef in den USA und bestätigte einige der ihm zur Last gelegten Vorwürfe, behauptete aber zugleich eine Tätigkeit als Doppelagent für die CIA. Vgl. RAFIZADEH, Witness, S. 99-12; eine weitere Bestätigung über verdeckte SAWAK-Aktionen gegen Oppositionelle im Ausland ergab sich infolge der Besetzung des iranischen Generalkonsulats in Genf durch oppositionelle Studenten im Juni 1976. Die Studenten erbeuteten belastende Dokumente und veröffentlichen diese später. Vgl. SHANNON, Losing Hearts and Minds, S. 288f.; MEIER, Helvetiens guter Draht, S. 243-58, 272-7; die ostdeutsche Staatssicherheit interessierte sich noch Jahre später für den Vorfall. Vgl. die Übersetzung aus dem Französischen, 22.10.86, BStU, MfS, HA XXII, Nr. 16692; siehe CONFEDERATION OF IRANIAN STUDENTS (NATIONAL UNION), Documents on Iranian Secret Police SAVAK, o.O. 1976.

${ }^{262}$ Vgl. Tehran \#241, 10.1.77, FRUS 69-76, XXVII, S. 595-8; vgl. SHANNON, Losing Hearts and Minds, S. 2918; zur zunehmenden Einflussnahme des Kongresses in der Post-Vietnam-Ära vgl. SCHMITZ, Right-Wing Dictatorships, S. 112-42; für eine frühe Eingabe mit Kritik an der Repression in Iran von Mitgliedern des USRepräsentantenhauses an die Administration vgl. Mitchell u.a. Letter to Kissinger, 3.12.73, POL 23-9 IRAN, CF 70-73; vgl. Smith Memo, 9.11.76, DNSA: IR01122.
} 
Hohn anstelle der gewünschten Anerkennung als Bannerträger aufgeklärten Gedankengutes. Das Engagement der Zwillingsschwester des Schahs, Ašraf, in der internationalen Menschenrechtspolitik - die „Karriere“ erlebte 1970 den Höhepunkt ihre Wahl zur Vorsitzenden der United Nations High Commission for Human Rights - bot zudem eher Gelegenheiten für die oppositionelle Studentenschaft im Ausland, die internationale Aufmerksamkeit auf die Repression in Iran und die Doppelmoral der regierenden Dynastie zu lenken. $^{263}$

Trotz politischer Stagnation, der neuartigen terroristischen Bedrohung und der negativen internationalen Aufmerksamkeit für die Herrschaftsmethoden in Iran ist der Eindruck irreführend, die interne Stabilität in Iran wäre zu dieser Zeit in Washingtoner Regierungszirkeln ernsthaft in Frage gestellt worden. Die gerade aufgeführten Faktoren waren auch in ihrer Gesamtheit nicht in der Lage, die weiterhin positive, in manchem fast euphorisch zu nennende Wahrnehmung der Fortschritte in Iran zu erschüttern. Pahlawi-Iran war Anfang der 1970er Jahre aus Sicht des atlantischen Wertekanons weiterhin exemplarisch für die Erfolgsaussichten einer staatlich gelenkten Modernisierung nach westlichem Vorbild. Kissinger selbst bezeichnete Iran als , an island of stability in an otherwise unstable area“ ${ }^{264}$ Bezeichnend für die herausragenden Erfolge des Schahs, so die Botschaft in einer Einschätzung von Anfang 1973, sei die Abwesenheit ,[...] of any group which conceivably pose a threat to his regime“:

The political parties, Majlis, judiciary and other organs of government and the press are closely controlled; the growing middle class is kept reasonably satisfied through economic rewards; the power of the conservative landlords was shattered by the White Revolution; the traditional clergy-bazaari alliance could cause trouble but lacks the clout to threaten the regime; the military appears to be loyal; the tribes could cause only extremely localized problems; and the students and terrorists lack a broad base of support, are not well organized, and live in fear of the ruthless and efficient security organization, SAVAK. So long as the Shah is alive and retains both possession of his faculties and the loyalty of the military and security organs, there is little chance that any group or individual could threaten his reign or even substantially weaken his power. $^{265}$

Das Regime war aus Sicht von Botschaft und auch der CIA im Grunde unangreifbar. Symptomatisch für diese Überzeugung war die Feststellung in einer Geheimdienstanalyse zur möglichen Nachfolge Mohammad Rezās noch im August 1978, also bereits nach Ausbruch erstzunehmender Unruhen in dem Land: „Iran is not in a revolutionary or even a

\footnotetext{
${ }^{263}$ Zur Konferenz von 1968 vgl. BuRKE, International Human Rights, S. 92-111; SHANNON, Losing Hearts and Minds, S. 247-51.

${ }^{264}$ Kissinger Memo, 16.4.70, FRUS 69-76, E-4, doc. 62 \{Hervorheb. v. Verf.\}.

${ }^{265}$ Tehran \#A-4, 9.1.73, FRUS 69-76, XXVII, S. 3; insbesondere die Tudeh-Opposition sei praktisch in Auflösung begriffen. Vgl. Tehran \#A-180, 17.4.69, POL 12 IRAN, CF 67-69; CIA, National Intelligence Survey Ausschnitt: ,Communist Subversion in Iran', Nov. 1968, DNSA: IR00698; die vormaligen Hauptträger oppositionellen Gedankenguts, die Mittelklassen, seien inzwischen - abgesehen von einigen Intellektuellen und Studenten - ,the backbone of the monarchical system as it functions today."; Tehran \#A-123, 8.7.75, Asnäd 7, S. 154-73, hier S. 159; darunter seien in der Tat auch viele ehemalige Aktivisten und Parteigänger der nationalistischen Opposition zu finden: „In fact, one of the Shah's major achievements of the past 12-15 years has been to wean former malcontents in supporting positions."; ebd.
} 
'prerevolutionary’ situation.“‘266

Wie bereits Mitte der 1960er Jahre war die Hauptsorge das unerwartete Ableben der zentralen Person im iranischen Herrschaftssystem: Mohammad Rezā Šāh. Aber anders als Mitte der 1960er Jahre war man in der darauffolgenden Dekade optimistischer, was die Nachfolgeregelung bzw. ihre Durchsetzung anging. Im Normalfall erwarteten die Botschaft wie die CIA ein komplikationsfreies Fortdauern der Pahlawi-Herrschaft bis zur Volljährigkeit von Kuroš Rezā Pahlawi im Jahr 1980 und somit das Ausbleiben eines Nachfolgestreites. ${ }^{267}$ Angesichts der erkannten Zentralität Mohammad Rezās für die Stabilität Irans wie auch des bilateralen Verhältnisses muss es umso mehr verwundern, dass keine eingehenden medizinischen und psychologischen Studien des Herrschers angefertigt wurden, was man auch innerhalb der CIA nach der Revolution verblüfft feststellte. ${ }^{268}$ Offenbar hatte man auf amerikanischer Seite tatsächlich keine Informationen über die Krebserkrankung Mohammad Rezās - Gary Sick nannte sie später ,,[...] one of the best-kept state secrets of all time.“269 Offenbar unbemerkt vom US-Geheimdienst unternahmen die französischen Spezialisten Jean Bernard und Georges Flandrin 1974 die erste von 35 geheimen Reisen nach Iran, um die sich stetig verschlimmernde Krebserkrankung Mohammad Rezās zu behandeln. ${ }^{270}$ Die amerikanischen Notfallszenarien aus dieser Zeit beschäftigten sich somit eher mit einem geglückten Attentat auf den Herrscher oder dessen Unfalltod.

Für den Fall eines vorzeitigen Ablebens Mohammad Rezās und der Einsetzung eines Regentschaftsrates unter Leitung von Farah Dibā sei aber trotz der Aufwertung der Rolle der Šahbānu in jüngerer Zeit mit einigem Widerstand zu rechnen, gerade vonseiten der Zwillingsschwester und erklärten Feindin Farahs, Ašraf Pahlawi, und der ihr zugeneigten

\footnotetext{
${ }^{266}$ CIA, National Foreign Assessment Center Intelligence Assessment: ,Iran After the Shah', August 1978, CREST: \#RDP80*001-6; allerdings häuften sich ab Mitte der 1970er Jahre die Berichte über wachsende Unzufriedenheit in weiten Teilen der Bevölkerung und selbst unter den Staatsbediensteten. Vgl. CIA Intel Information Cable, 8.5.75, FRUS 69-76, XXVII, S. 344ff.; das NIE von 1975 versuchte dem allgemeinen Trend, die Opposition in Iran zu bagatellisieren, zumindest etwas entgegenzutreten. Vgl. NIE 34-1-75: ,Iran', 9.5.75, FRUS 69-76, XXVII, S. 346-67, hier 351-4; der Nachrichtendienst der U.S. Air Force widersprach resolut in einer abweichenden Meinung in einer Fußnote. Das NIE überzeichne, the extent and significance of dissent in Iran and prejudges the Shah's capacity to effect successful reforms."; ebd., S. $354 n 2$; für die interessante These, das es gerade die unbestrittene politische Dominanz des Schahs und des Hofes gewesen sei, die später jeden denkbaren Widerstand gegen die Revolutionäre von 1978/79 untergrub, vgl. KANDIL, Power Triangle, S. 35-135.

${ }^{267}$ Vgl. Tehran \#A-4, 9.1.73; NIE 34-1-75: ,Iran', 9.5.75, FRUS 69-76, XXVII, S. 1-19, S. 354f., ähnlich bereits die CIA 1972: „Short of assassination or a sudden illness, the Shah will probably continue his present style of ruling for perhaps as much as two decades.“; CIA Intel Report 2035-72 \{,Centers of Power in Iran'\}, Mai 1972, FRUS 69-76, E-4, doc. 180; seit dem Aufstieg der Gāăăren-Dynastie im Jahr 1795 war es von neun Schahs nur zweien vergönnt, auf dem Thron eines natürlichen Todes zu sterben, warnte allerdings die CIA. Vier Herrscher wurden abgesetzt, eine Dynastie komplett ausgetauscht und zwei fielen Attentaten zum Opfer. Zu den erwarteten Akzeptanzproblemen der Regentschaft vgl. bereits CIA, NIE 34-69 \{,Iran'\}, 10.1.69, ebd., doc. 1.

${ }^{268}$ Vgl. Senior Review Panel Memo to DCI, 6.1.84, CREST: \#RDP86*006-2; vgl. Precht Letter, 10.10.78, DNSA: IR01581; TelCon, 1.6.76, State:HKTT.

${ }^{269}$ SICK, All Fall Down, S. 182; allerdings war man aber aufmerksam, was mögliche Attentatsgefahren anging. Offenbar unternahm man 1973 nicht weiter ausgeführte Sicherheitsmaßnahmen vor Ort. Vgl. TelCon, 15.3.73, DNSA:HAKTelCons: \#KA09770.

${ }^{270}$ Der Schah litt unter einer chronischen lymphatischen Leukämie. Vgl. BuCHAN, Days of God, S. 187-90; Shawcross, Shah's Last Ride, S. 230-52; ZONIS, Majestic Failure, S. 150-65; KhoshnOod/KHoshnOOD, Political Cancer; vgl. Tehran \#7043, 26.7.78, DNSA: IR01449.
} 
Höflinge. Zwar sei Farah weitaus populärer bei der iranischen Bevölkerung als ihre Kontrahentin, doch war man sich unsicher, ,[...] that she has the intestinal fortitude necessary to ward off threats to her position. “271 Entscheidend in diesem Szenario wäre aber das Militär, das als einzige Institution die nötige Einigkeit und Organisation besäße, die politischen Geschicke des Landes zu steuern. Der als „extremely pro-American“ beschriebene Luftwaffenchef Mohammad Xātami wurde als wahrscheinlichste Führungsfigur identifiziert, was die Sorgen auf amerikanischer Seite bis zu Xātamis Unfalltod 1975 besänftigte. Allgemein wurden die Streitkräfte von der Botschaft als verlässlich pro-amerikanisch eingeschätzt: „Our long and close collaboration with the military elite should pay dividends during an uncertain period of political transition." ${ }^{272}$

Ein revolutionärer Sturz des Pahlawi-Regimes lag aber außerhalb der Vorstellungswelt der allermeisten amerikanischen Iranbeobachter. Ein hoher Iran-Analyst in der CIA brachte die vorherrschende Einstellung Anfang 1976 zum Ausdruck: „The Shah of Iran has been on his throne 34 years, far longer than any other leader in the Middle East. He has not only outlived most of these rulers, but has outlasted the many official and unofficial observers who, two decades ago, were confidently predicting his imminent downfall. “273 Wenngleich es leicht fällt, ex post facto diese Einstellung als naiv zu verdammen, so kann doch die Überzeugungskraft dieser Argumentation in den 1970ern kaum bezweifelt werden. In der Tat hatten sich viele frühere Kritiker Mitte der 1970er Jahre mit der nun als resilient eingeschätzten PahlawiHerrschaft arrangiert - Kritiker will Bill Miller oder William Polk waren in die Akademie zurückgekehrt, weder im Weißen Haus noch in State Department fanden sich kritische Stimmen, zumal Zweifel an dem als wichtigster nahöstlicher Verbündeten eingeschätzten Schah nicht unbedingt karriereförderlich waren. ${ }^{274}$ Die Botschaft unter Richard Helms gebärdete sich dabei zunehmend als Parteigänger der Pahlawi-Herrschaft und versuchte schon im Ansatz jede kritische Berichterstattung zu ersticken bzw. jede negative Prognose unter

\footnotetext{
${ }^{271}$ Tehran \#A-4, 9.1.73, FRUS 69-76, XXVII, S.1-19, hier 8.

${ }^{272}$ Ebd., S. 13; die CIA sah allerdings Gefahren für das Überleben des monarchischen Systems im Falle eines erfolgreichen Attentats auf den Schah aufgrund der vorherrschenden Stimmungen unter den Offizieren niedrigeren Ranges. Vgl. CIA; NIE 34-1-75: ,Iran', 9.5.75, FRUS 69-76, XXVII, S. 346-67, hier S. 354f.; ein INR-Offizieller kritisierte die vorhandenen amerikanischen Contingency-Pläne für das Szenarios eines Ablebens als zu pessimistisch. Insbesondere wehrte er sich gegen die etablierte Vorstellung, ,that the U.S. will have virtually no chance to influence events after the Shah departs.“; zudem glaubte er, dass es unter gewissen Konstellationen wie z.B. einem Bürgerkrieg mit linksgerichteten Kräften durchaus auch zu einer militärischen Intervention der USA kommen könnte, was in den bisherigen Plänen aber als Option fehlte. Vgl. Childs Memo, 12.8.70; in Bezugnahme auf Contingency Plan: ,Proposed Action by the United States in the Event of the Death of the Shah', o.D., Lot 80 D 234, ASS/NEA: Top Secret Records 1965-75, Box 1; vgl. Tehran \#A-90, 6.5.76, DNSA: IR01051.

${ }^{273}$ CIA Study ,Analysis of NFAC's Performance on Iran's Domestic Crisis, Mid-1977 - 7 November 1978‘, 15.6.79, CREST: \#RDP86*002-5, S. 11.

${ }^{274}$ Botschafter Meyer berichtete süffisant von einer Begegnung mit Kenneth R. Hansen am Rande einer Rede des Schahs an der University of Chicago. Hansens Ansichten hätten sich sehr gewandelt, stellte Meyer fest: „Undoubtedly rich monetary rewards can also have their mellowing effect, even upon intellectuals."; Meyer Letter, 5.3.69, DEF 12-5 IRAN, CF 67-69; selbst ein kritischer Geist wie Bill Polk schien den finanziellen Verlockungen der Pahlawi gegenüber aufgeschlossen. Ein Brief der Botschaft erwähnte Polks Absicht, den Schah um finanzielle Unterstützung für sein neues universitäres Institut zu bitten. Allerdings findet sich in den Akten kein eigentlicher Beleg für eine solche Anfrage. Vgl. Herz Letter, 23.3.66, ,POL 7 Visits. Missions. 1966', Lot 70 D 330, NEA/IRN: 1964-66, Box 18; zur Rolle der Wissenschaft in dieser Zeit vgl. SHANNON, Education.
} 
Berufung auf die überlegene Informationslage vor Ort zu diskreditieren. Gerade unter Botschafter Richard Helms (April 1973-Dezember 1976) mit seiner langjährigen persönlichen Nähe zum Herrscher machte sich dieser Trend bemerkbar, aber bereits unter seinen Vorgängern Joseph S. Farland (Mai 1972-März 1973) und Douglas MacArthur II (Oktober 1969-Mai 1972) war die Localitis allgegenwärtig, ein Schmähbegriff Kissingers für die allzu große Nähe zwischen den Positionen amerikanischer Botschaften und den Gastregierungen vor Ort. ${ }^{275}$ So widersprach die Botschaft im Sommer 1975 vehement einer nur ansatzweise kritischeren Geheimdiensteinschätzung zu Iran wegen ihres unzutreffenden ,rather alarmist portrayal of \{the; R.P.\} Iranian political situation.“"276

Einflüsse von außerhalb der Regierungsbürokratie boten wenig Alternativen für diese unisone Sichtweise. Der wichtigste Verbündete in der Nahostregion, Großbritannien, setzte noch bedingungsloser auf den Schah als Garanten der Stabilität in Iran - der britische Botschafter Denis Wright machte seinem amerikanischen Kollegen bereits 1967 klar, dass ,[...] as far as he and his country are concerned all interest is centered in keeping the Shah happy.“277 Während die Kennedy-Administration Anfang der 1960er Jahre noch auf kritische Einschätzungen der Lage in Iran seitens amerikanischer Nahostspezialisten an Universitäten und Denkfabriken zurückgreifen konnte, hatte sich die Forschungslandschaft inzwischen stark gewandelt. Opportunistische Interessen und die Generosität des Pahlawi-Hofes hatten in den universitären Landschaften Nordamerikas und Westeuropas einen servilen Resonanzkörper für dynastische Selbstdarstellung geschaffen. Lobpreisungen für Mohammad Rezā als weitsichtigen Reformer und Staatsmann und als Höhepunkt der monarchisch geprägten Geschichte Irans fanden bis in die 1970er Jahre große Aufmerksamkeit. Iran-Experten wie der Pahlawi-Claqueur George Lenczowski oder der ehemalige CIA-Agent Donald Wilber übertönten kritischere Stimmen, und nur wenige Wissenschaftler solidarisierten sich mit den oppositionellen iranischen Studenten. ${ }^{278}$

\footnotetext{
${ }^{275}$ Gerade MacArthur II übertraf die bereits beträchtliche Willfährigkeit seiner Vorgänger seit Julius Holmes und unterstützte - in der Regel vorbehaltlos - die verschiedenen, meist militärischen Wünsche und Forderungen des Schahs. Vgl. Tehran \#1247, 1.4.70, FRUS 69-76, E-4, doc. 57; ähnlich Tehran \#3144, 22.7.70, ebd., doc. 78; MacArthur II zitierte sogar die alte Beschwerde Meyers über die kontraproduktive „"pappa knows best» attitude“, die angeblich immer noch in Washington existiere. MemCon, 8.4.71, POL IRAN, CF 70-73; vgl. die eingehende Analyse der Botschaft bei BILL, Eagle and Lion, S. 382-401.

${ }^{276}$ Es handelte sich um eine Kritik am bereits erwähnten NIE von 1975. Siehe Tehran \#5850, 19.6.75, FRUS 6976, XXVII, S. 407.

${ }^{277}$ Wright gab sich überzeugt ,that the UK should put all its Mideast chips on the Shah."; Meyer Letter, 16.3.67, FRUS 64-68, XXXIV, S. 376; zur fortgesetzt devoten britischen Haltung gegenüber Iran und Mohammad Rezā vgl. PosnetT, British Policy; vgl. LuCAS, Decline and Fall; die Dominanz kommerzieller Motive auf britischer Seite bei ALI, British Diplomacy, S. $56 \mathrm{ff}$.

${ }_{278}$ Beispielhaft war der servile Sammelband Iran Under the Pahlavis aus dem Jahr 1978, herausgegeben von Lenczowski, wo er im Vorwort die iranische Geschichte als ,continuous struggle of strong-willed rulers to elevate their nation from the level of weakness and backwardness to a higher level of strength and modernity" interpretierte. Ebd., S. xxii. Vgl. auch seine Apologetik zur Repression in dem selben Band. LENCZOWSKI, Second Pahlavi Kingship; Lenczowksi nutzte seine Verbindungen auch zur persönlichen Bereicherung. Die Botschaft beschrieb seine jährlich wiederkehrenden Nahostbesuche in erster Linie mit der Abfassung eines ,,consulting report" für den ESSO-Konzern begründet, ,, a fact which he prefers not to broadcast“; Tehran \#A-382, 3.9.69, POL 15-1 IRAN, CF 67-69; eine kritische Bestandsaufnahme der gegenüber der Pahlawi-Autokratie unkritischen bzw. häufig devoten Forschungslandschaft in den USA vor dem Ausbruch der Revolution bei RICKS, Academics in the Service; vgl. BILl, Eagle and Lion, S. 373f.; vgl. jetzt auch SHANNON, American Academics, der eine ,kritische
} 
Erst Mitte der 1970er Jahre fand die Gegenöffentlichkeit mit ihrer Kritik an Repression und Menschrechtsverletzungen in Iran, getragen vorwiegend von Exiliranern und der oppositionellen Studentenschaft, und unterstützt von den Ausläufern der New Left, größere Aufmerksamkeit gerade in den US-Medien, und in Reaktion darauf, auch im amerikanischen Kongress. Hier ist auch der Beginn des graduellen Einflussverlustes der Pahlavists zeitlich zu verorten, die ihren Höhepunkt in der vorangegangenen zehn Jahren durchlebten und eine Art Paralleldiplomatie zwischen Teheran und Washington unterhielten, die immer auch zum eigenen Vorteil gereichte. Überzeugte Pahlavists wie Kermit Roosevelt, David E. Lilienthal und die Rockefeller-Brüder arrangierten Kontakte in die jeweiligen Administrationen und die Wirtschaftselite, sorgten sich um eine genehme Berichterstattung mittels ihrer Kontakte zu führenden Journalisten und Herausgebern, und profitierten zugleich pekuniär von ihrer Rolle als Mittelsmänner zum Hof. ${ }^{279}$ Die iranische Botschaft in Washington sorgte mit rauschenden Festen und generösen Geschenken, darunter die obligatorischen Kaviarpakete, für Wohlwollen in den politischen und medialen Eliten Washingtons. ${ }^{280}$ Trotz der Schwächung der Pahlavists angesichts des gewandelten Zeitgeistes nach der Vietnam-Niederlage sollte sich ihr Einfluss ein letztes Mal während der Revolution im Kontext der Einreise des Schahs in die USA bemerkbar machen, dann allerdings mit fatalen Folgen für das bilaterale Verhältnis.

Eine Konstante in Expertenmeinungen zur Fortentwicklung Irans und des Verhältnisses zu den USA war die Überzeugung, dass die in den vorangehenden Jahrzehnten aufgebauten vielfältigen Verbindungen zwischen beiden Staaten und Gesellschaften ein Fundament errichtet hätten, das auch größeren Verwerfungen standzuhalten in der Lage wäre. ${ }^{281}$ Zudem würden „geopolitische“ Realitäten ein Festhalten am engen Verhältnis zu Washington unumgänglich machen, unabhängig von der politischen Orientierung einer Regierung in Teheran, solange sie nicht einseitig kommunistisch dominiert werde. Der im vorherigen Kapitel erwähnte

\footnotetext{
Minderheit" in der US-Akademie identifiziert. Angesichts der Aufgabe wissenschaftlicher Prinzipientreue durch diese Forscher muss die im Anschluss von 9/11 versuchte Rehabilitierung dieser Forschungsrichtung durch rechtsund neokonservative Kreise in den USA erstaunen. Infam die Intention, die amerikanischen Middle Eastern Studies en bloc zu diskreditieren, offenkundig mit der Absicht kritische Stimmen vor dem Irakkrieg von 2003 verstummen zu lassen, bei KRAMER, Ivory Towers on Sand.

${ }^{279}$ Zum Netzwerk weiterhin grundlegend BILL, Eagle and Lion, S. 319-78; andere US-Offizielle traten sogar in den iranischen Staatsdienst über. Der ehemalige OSS-Agent Taylor Gurney war in den 1960er Jahren in der iranischen Botschaft in Washington angestellt. Vgl. Blosser Letter, o.D. [1962], ,2500[...]Empire 8`, Lot 69 D 30 , NEA/IRN: 1964-66, Box 2.

${ }^{280}$ David RoCKEFELLER, Memoirs, S. 385, behauptete später angesichts der Langzeitfolgen, den Schah nur flüchtig gekannt zu haben. So habe er ihn in den 1960er Jahren nur zwei Mal persönlich getroffen. Das Gros seiner Lobbytätigkeiten fiel allerdings in die darauffolgende Dekade. Vgl. dagegen BILL, Eagle and Lion, S. 180f., 31923; ein weiterer Faktor war die immer wieder kolportierte Korruption. SAWAK-Quellen scheinen z.B. zu belegen, dass sich der ehemalige CIA Station Chief Yatsevich im Anschluss an seine Karriere in zweifelhaften Geschäften mit Iran bereichert habe. Vgl. NASR, Late-Pahlavi State, S. 103f.; MiLANI, Persian Sphinx, S. 143; auch Richard Helms bemühte sich offenbar nach seiner Ablösung als Botschafter um lukrative Kontrakte.

${ }^{281}$ So auch die CIA 1975: „While US-Iranian relations would come into question if the Shah should leave the scene, much of the relationship has become institutionalized to the point where it transcends the Shah. [...] But even a more extreme regime probably would not immediately seek to restructure the present relationship in drastic ways given the Iranians' view of their interests and the importance of the US connection."; NIE 34-1-75 \{Iran\}, 9.5.75, ebd., S. 348 .
} 
Contingency Plan für den Fall einer Beseitigung des Schahs war stark beeinflusst von der Erwartung, dass mit einem Nachfolgeregime zu rechnen sei, welches sehr wahrscheinlich antiamerikanische Züge trage. Hauptziel in einer solchen Phase müsse es daher sein, „[...] whatever the events in Iran, our strategy be designed to preclude the introduction of foreign troops onto Iranian soil and, though it may be difficult to ride through turbulent periods during which the U.S. may lose influence and perhaps even be vilified, to seek to maintain our influence insofar as circumstances permit.“282 Hintergedanke hinter dieser Empfehlung war eine spezifische Interpretation der iranischen Geschichte, die eine Kontinuität in dem Streben nach territorialer und politischer Integrität unabhängig vom Charakter des jeweiligen Herrschaftssystems identifizierte. Somit würde auch ein zukünftiges anti-westliches Regime aufgrund geopolitischer Gegebenheiten früher oder später die USA um Hilfe bzw. Unterstützung anrufen. ${ }^{283}$ Dieses geopolitische Axiom sollte in der Tat im Anschluss an die iranische Revolution von 1978/79 lange handlungsbestimmend für die amerikanische Außenpolitik bleiben und selbst nach der Botschaftsbesetzung gab es weiterhin Protagonisten auf amerikanischer Seite, die an der Validität dieser Annahme festhielten, was später direkt zur Blamage des Iran-Contra-Skandals führen sollte. ${ }^{284}$

Eng verbunden mit der Hypothese einer ,geopolitisch“ determinierten Permanenz enger Beziehungen zwischen Iran und den USA war die Überzeugung, dass nur ein pro-sowjetisches Regime dauerhaft anti-amerikanischen Stimmungen nachgeben würde. Zwar hatte man den seit Mitte der 1960er Jahre ansteigenden Anti-Amerikanismus in Iran durchaus registriert, doch wurde dem Phänomen letztlich nicht die ihm zukommende Beachtung geschenkt. Die Besorgnis wuchs in der zweiten Hälfte der 1960er Jahre. Neben der sich international verflechtenden iranischen Wirtschaft sorgte gerade die militärische Aufrüstung für ein fortwährendes Ansteigen der amerikanischen Präsenz in Iran. Bereits 1968 drängte Mohammad Rezā auf eine Vergrößerung von ARMISH/MAAG um 200 zusätzliche Flugzeugtechniker für die Wartung der auszuliefernden hochmodernen F-4-Kampfflugzeuge. ${ }^{285}$ Die Frage zusätzlichen amerikanischen Bodenpersonals für die Luftwaffe beschäftigte beide Seiten in den Folgejahren und wurde schließlich im Rahmen des Nixon-Kissinger-Besuchs im Mai 1972 im Sinne Mohammad Rezās entschieden. Die Zahl von 1972 in Iran anwesenden 605

\footnotetext{
${ }^{282}$ Contingency Plan: ,Proposed Action by the United States in the Event of the Death of the Shah ', o.D., ,Iran', Lot 80 D 234, ASS/NEA: Top Secret Records 1965-75, Box 1.

${ }_{283}$ Ähnlich die Botschaft drei Jahre später: „Moreover, these geopolitical imperatives would, in our view, be likely to reassert themselves even if, as we consider most unlikely, this dynasty should disappear. For we believe that whatever group eventually succeeded to power, perhaps after a period of greater or lesser instability, would be of an Iranian nationalist, rather than Communist, orientation, at least in its foreign relations. Any regime dedicated to promoting essentially Iranian objectives would have to look askance at the Soviet Union just as the present one does. Thus, the felt Iranian need for the United States to counterbalance the Soviet Union would remain and the essential basis for our continuing cooperation would remain. "; Tehran \#A-4, 9.1.73, FRUS 69-76, XXVII, S. 16f.; vgl. auch Tehran \#2488, 1.5.72, FRUS 69-76, E-4, doc. 182.

${ }^{284}$ Als Leitthema bei EMERY, US Foreign Policy and the Iranian Revolution; ähnlich die Argumentation im Anschluss an die Revolution bei RUBIN, U.S. Options.

${ }^{285}$ Vgl. Newcomer Memo, 12.6.68, FRUS 64-68, XXII, S. 523ff.; vgl. TelCon, 23.10.69, DNSA:HAKTelCons: \#KA01427.
} 
amerikanischen Militärangehörigen stieg in der Folge der Entscheidung rapide an. ${ }^{286} \mathrm{Im}$ Oktober 1973 warnte die US-Botschaft vor einer weiteren Zunahme der Präsenz von nunmehr 1.600 Botschaftsangehörigen, Militärberatern und Technikern (,blue-suiters“) und den bereits 2.000 anwesenden, zivilen amerikanischen Angestellten im Rahmen des Rüstungsprogramms. Zwar seien die meisten Iraner im Allgemeinen gegenüber Amerikanern nicht feindselig eingestellt, doch seien ,latent tensions in some quarters of population“ nicht von der Hand zu weisen. ${ }^{287}$ Ein Jahr darauf versuchte die Botschaft unter Helms dann aber die wachsende Besorgnis zu zerstreuen und konstatierte ein ,very positive desire for foreign expertise“ in den Führungsschichten und weitgehende Indifferenz beim Gros der Bevölkerung. Nur bei einigen wenigen Studenten gebe es Widerstand gegen die wachsende US-Präsenz, behauptete Helms: „Although resentment may grow with enlargement of foreign community, we do not foresee a serious and organized direct threat to U.S. presence and do not believe we should unduly inhibit essential [...] defense-related activities in Iran in fear of popular reaction." ${ }^{288}$ Tatsächlich war die Entsendung von Helms nach Teheran umstritten gewesen. Angesichts der weithin bekannten Involvierung der CIA im Coup d'état von 1953 war die Entsendung eines ehemaligen Director of Central Intelligence aus Sicht vieler Iraner nur ein weiterer Beleg für die Steuerung iranischer Politik durch ausländische Kräfte. Die weitgehende Emanzipation Irans von amerikanischer Dominanz im vorausgegangenen Jahrzehnt änderte offenbar nur wenig an der Persistenz solcher Wahrnehmungen. ${ }^{289}$ Entgegen der dominierenden Vorstellungen über amerikanische Omnipotenz in Iran waren die Informationsquellen der CIA offenbar vergleichsweise begrenzt - angesichts des Charakters des iranischen Herrschaftssystems und der Dominanz des Schahs waren Manipulation von außen zudem enge

\footnotetext{
${ }^{286}$ Nach einem Verweis des Schahs auf die angebliche Präsenz von 10.-20.000 sowjetischen Technikern in der VAR sagte Nixon ,any number that is needed in Iran“ zu. Vgl. Saunders Memo, 12.6.72, FRUS 69-76, E-4, doc. 204; einige Monate später ging man von der Entsendung von etwa 2.500-3.000 Technikern aus, was, Familienangehörige eingeschlossen, allein die militärbezogene US-Präsenz auf etwa 10.000 Personen vergrößert hätte. Bald reduzierte sich die Zahl aber auf etwa 900 Mann, vorwiegend im Rahmen von gemischt zivilmilitärischen Technical Assistance Field Teams (TAFTs). Eine entsprechende Vereinbarung erfolgte im Januar 1973. Vgl. Saunders Memo, 2.8.72; Nutter Memo, 28.12.72, ebd., docs. 216, 243; Saunders Memos, 6./19.1.73, ,Iran, Vol. IV, 1 Sep 71-Apr 73“, NSCF, Country Files-Middle East, Box 602, RMNL; vgl. auch Saunders Memo, 26.1.73, FRUS 69-76, XXVII, S. $27 \mathrm{f}$.

${ }^{287}$ Vgl. ed. note, ebd., S. 144f.; Anlass der Warnung der Botschaft waren Pläne für die Verlegung einer sensitiven Kommunikationsstation aus Kagnew/Äthiopien nach Iran inklusive des Personals von 80-100 Mann. Ende 1974 berichtete die Botschaft über „culture difficulties“ im konservativen Isfahan infolge der neuen Präsenz von Technikern von Bell Helicopter. Siehe ebd., S. 281 n2; vgl. Tehran \#7200, 27.7.75, DDRS: CK3100583138.

${ }^{288}$ Tehran \#10619, 16.12.74, FRUS 69-76, XXVII, S. 278; die Botschaft beschränkte ihre Analyse auf die Haltungen der etwa 15\% ,gebildeten Schichten“ und ignorierte bewusst die Masse der Bevölkerung. Zu diesem Zeitpunkt waren laut Botschaft 1.000 US-Militärberater und 1.900 zivile Beschäftigte im Aufrüstungs- und Trainingsprogram beschäftigt. Man rechnete allerdings mit einem Anstieg der letzteren auf etwa 12.000 Personen bis 1980. Die amerikanische „Community“ in Iran war insgesamt auf etwa 16.000 Personen angestiegen. Allerdings umfassten sogar die westdeutschen, britischen und sowjetischen Gemeinden in Iran jeweils etwa 5.000 Personen. Tatsächlich meldeten sich auch in der Botschaft besorgte Stimmen. Siehe Winkler Memo, 24.11.75, ebd., S. 444-7; vgl. auch DOD Report, , The Growing U.S. Involvement in Iran“, 22.1.75, DNSA: IR00927.

${ }_{289}$ Entsprechende Kommentare fanden sich auch in US-Zeitungen. Siehe z.B. ,Helms, the Shah and the CIA', Washington Post, 29.12.72, CREST: \#RDP80*001-5; zur Wahrnehmung in Iran vgl. BILL, Eagle and Lion, S. 213; die häufig kolportierte Geschichte, Helms und Mohammad Rezā hätten sich bereits aus gemeinsamen Schultagen im Internat Le Rosey am Genfer See gekannt, sind offenbar unzutreffend. HELMS, Look over my Shoulder, S. 14f. zufolge traf der Schah erst nach Ablauf seiner Zeit am Internat ein. Er habe Mohammad Rezā zum ersten Mal 1957 persönlich kennengelernt. Zur Schulzeit des Schahs in der Schweiz siehe MEIER, Helvetiens guter Draht, S. 211-5; zur Ernennung vgl. auch TelCon, 28.11.72, DNSA:HAKTelCons: \#KA09094.
} 
Grenzen gesetzt. ${ }^{290}$

Nicht jeder in der US-Administration teilte die Nonchalance der Botschaft. Ein Memorandum aus dem DOD von Anfang 1975 zeigte sich besorgt über die Verdreifachung der US-Präsenz mit militärischen Bezug in Iran seit Anfang der Dekade auf insgesamt 17.000 Personen und einen absehbaren Anstieg auf etwa 50.000 bis 1980. Secretary of Defense Schlesinger nutzte die gestiegene Präsenz in seiner bereits geschilderten Attacke auf die generelle Iranpolitik der Ford-Administration. Schlesinger warnte den Präsidenten, das rasante Anwachsen der amerikanischen Gemeinde könne Anti-Amerikanismus und Terrorismus provozieren: „If the political situation turned sour it could leave us very vulnerable. “291 Die iranische Stadtguerilla hatte in der Tat bereits früh begonnen, amerikanische Offizielle und Militärberater ins Ziel zu nehmen. Bereits Ende 1970 hatte eine Gruppe versucht, den damaligen US-Botschafter MacArthur II zu entführen. Im Juni 1973 wurden ein und im Mai 1975 zwei weitere Angehörige von ARMISH/MAAG, im August 1976 dann drei zivile amerikanische Militärtechniker ermordet. Hinzu kam eine ganze Serie von Bombenanschlägen gegen amerikanische Einrichtungen, zwei Mal davon gegen die Botschaft selbst. ${ }^{292}$ Viele USOffizielle registrierten durchaus, dass es inzwischen einen festen Bodensatz antiamerikanischer Stimmungen in breiten gesellschaftlichen Schichten gab, doch interpretierte man die Gewalttaten als Ausnahmeerscheinungen einiger weniger radikalisierter Individuen. Angesichts der engen Bindung mit der pro-amerikanischen Elite schien die ,[...] majority of bazaar-connected Iranians who are deeply religious, largely conservative in social orientation and also generally anti-foreign in outlook" nicht weiter von Bedeutung zu sein. ${ }^{293}$

Wichtig für das Verständnis der US-Haltung ist, dass man den religiös-konservativen Kräften mit ihren als reaktionär empfundenen gesellschaftlichen Ordnungsvorstellungen nicht zutraute, eine langfristig bedeutende Rolle innerhalb der Opposition zu spielen. Im Ernstfall, so die allgemein geteilte Überzeugung, werde man es mit einem stark nationalistischen, vielleicht

\footnotetext{
290 Ähnlich RAMAZANI, Patterns of Influence, S. 149; gewisse Informationen waren dennoch verfügbar. So kannte die CIA bereits einige Tage vor den Wahlen zum 22. Mağles die künftige Zusammensetzung der Legislative, da sie über die Vorabkontrolle der Kandidatenliste durch den SAWAK auf Anordnung des Schahs im Detail informiert war. Vgl. CIA Intel Memo, 1.8.67, CREST: \#RDP79*001-9.

${ }^{291}$ Schlesinger-Zitat aus FRUS 69-76, XXVII, S. $424 n 1$; Schlesinger sprach gegenüber Ford bereits von einer gegenwärtigen Größenordnung von 100.000 Personen, zu diesem Zeitpunkt aber eine Übertreibung. Vgl. Sullivan Memo, 23.1.75, ebd., S. 299f;; vgl. DOD Report, 5.8.75, DNSA: IR00980; vgl. auch BILL, Eagle and Lion, S. 380ff.; vgl. RAND Study [Brian Jenkins u.a.], ,Numbered Lives: Some Statistical Observations from 77 International Hostage Episodes', August 1975, DDRS: CK3100039045; die Botschaft erstellte 1976 eine informelle Studie über die Einstellung der iranischen Bevölkerung zur gestiegenen US-Präsenz. Vgl. Stempel Memo, 27.4.76, DNSA: IR01047.

${ }^{292}$ Vgl. Tehran \#5142, 1.12.70, FRUS 69-76, E-4, doc. 102; im September 1971 berichtete MacArthur II von der Zerschlagung des für den Anschlag auf ihn verantwortlichen Kommandos durch den SAWAK. Vgl. MacArthur II Letter, 16.9.71, POL 15-1 IRAN; Tehran \#364, 19.1.72, POL 23-9 IRAN, CF 70-73; der Mord an einem Lieutenant Colonel 1973 wurde offenbar von einem religiösen Extremisten verübt. Die marxistisch-islamistischen „Volksmudschahedin“ bekannten sich durch Anrufe bei der Botschaft zum Anschlag von 1975 und begründeten die Tat mit der Ermordung von Bižan Ğazani und acht anderen Evin-Gefangenen durch den SAWAK. Vgl. Tehran \#3878/\#4788/\#8696, 5.6.73/21.5.75/28.8.76, FRUS 69-76, XXVII, S. 54-7; S. 53 n2, 390f., 560f.; vgl. BILL, Eagle and Lion, S. 191.

${ }^{293}$ Tehran \#10619, 16.12.74, FRUS 69-76, XXVII, S. 279; vgl. Tehran \#A-101, 23.3.70, POL 2 IRAN, CF 70-73.
} 
links gerichteten Nachfolgeregime zu tun haben. Die traditionelle Basar-Klerus-Allianz als Hauptträger erzwungenen politischen Wandels in Iran habe ihre historische Bedeutung eingebüßt, konstatierte die Botschaft bereits Anfang 1973. ${ }^{294}$ Bereits 1968 hatte eine eingehende Untersuchung des religiösen Feldes in Iran ähnliche Vorhersagen generiert - Bill Millers mahnende Einschätzungen aus dem Jahr 1965 über die Gefährlichkeit der klerikalen Entfremdung gerieten somit rasch wieder in Vergessenheit. Letztlich werde die Macht der schiitischen Kleriker im Zuge der durchgreifenden Modernisierung und Säkularisierung schwinden, so die einhellige Überzeugung in der ersten Hälfte der 1970er Jahre. Gewaltsame Proteste wie 1963 seien nicht mehr zu befürchten: „We feel that time has all but run out for further violent action on the part of the Mullahs." ${ }^{295}$ Entwicklungen im Bereich der Religion wurden nunmehr als eher obskure Randerscheinungen wahrgenommen. Mit dem Tode des anerkannten Marğa' Muhsin aṭ-Ṭabāṭabā'i al-Ḥakim im Jahr 1970 rückte erneut Ājatollāh Khomeini in den Mittelpunkt. Die eigentlich erstaunliche Tatsache, dass der prononcierteste Kritiker der Schah-Herrschaft offenbar von vielen, vielleicht sogar von der Mehrheit der anerkannten schiitischen Gelehrten als geeignetster Kandidat für die Marğa'ijja angesehen wurde, schien die US-Botschaft nicht weiter zu verunsichern. ${ }^{296}$ Im Gegensatz zur Pahlawitreuen US-Botschaft gab es innerhalb der CIA offenbar ein besseres Gespür für die Bedeutung des religiösen Widerstandes und die gegebenen Mobilisierungschancen des Klerus im Falle einer ernsthaften politischen oder sozialen Krise. Eine sehr kundige geheimdienstliche Einschätzung vom Mai 1972 erwähnte z.B. die beeindruckenden finanziellen Mittel, die dem Klerus bis vor kurzem zur Verfügung gestanden hätten - sie verfügten womöglich über ein jährliches Einkommen von etwa \$30 Millionen und ein bekannter Moğtahed habe jeden Monat \$200.000 an seine Anhänger ausgeschüttet. Das eigene Wissen über den schiitischen Klerus sei aber unzureichend, doch könnten nur etwa $10 \%$ der Kleriker als verlässlich regimetreu eingeschätzt werden - wenigstens $50 \%$ seien als ,outright opposition“ einzustufen, darunter nahezu alle angesehenen Groß-Ājatollāhs - die übrigen 40\% seien vorerst unentschlossene „fence-sitters“. Angesichts der traditionell quietistischen Grundhaltung der großen Mehrheit schiitischer Gelehrter war diese Beschreibung des klerikalen Widerstandes sicherlich eine Übertreibung. Dennoch muss das Desinteresse am religiösen Feld angesichts dieser

\footnotetext{
294 „Therefore, it appears that the days when a clergy/bazaari alliance could directly precipitate significant political change are over. They could support or oppose a particular claimant for power, but would be most useful in the period following a change at the top when their influence, especially that of the clergy, would be instrumental in urging public acceptance of a new ruler or rulers."; Tehran \#A-4, 9.1.73, ebd., S. 6; bereits 1970 hatte die CIA festgestellt, der konservative Klerus lehne die Schah-Herrschaft ab, aber ,they appear to be able to do little more than grumble."; SNIE 34-70, 3.9.70, FRUS 69-76, E-4, doc. 86.

${ }^{295}$,As the impact of modernization and development spreads in Iranian society, the complaints of anti-regime (and anti-US) clerics will probably become less important."; Tehran \#A-674, SOC 12 IRAN, 22.6.68, CF 67-69; der Botschaft zufolge seien nicht wenige religiöse Führer sogar pro-amerikanisch eingestellt.

296 Die Pahlawi-Monarchie unterstützte die Ansprüche auf die Marğa ’ïjja vonseiten der quietistischen Ājatollāhs Mohammad Kāzem Šari'atmadāri und Hāğğ Sejjed Ahmad Xonsāri. Šari atmadāris Kandidatur erfahre aber starken Widerstand, so die Botschaft. Khomeini werde nicht nur von der Regierung in Bagdad und seitens der kommunistischen Exilsender bevorzugt, sondern besäße viel Unterstützung in Iran selbst: „Khomeini has strong support among the bazaaris and Ulema in Tehran and his selection is also being encouraged by Iraq and the clandestine radios. His picture is being displayed in the bazaar and south Tehran and some Mullahs have begun reading the daily prayers in his name - an honor reserved for the leader of the Shiites."; Tehran \#A-217, 7.7.70, FRUS 69-76, E-4, doc. 76; angesichts der Uneinigkeit rechnete man mit einer Vakanz im Marğa'ijja.
} 
Einschätzungen überraschen, zumal man durchaus das gleichzeitige erhebliche sowjetische Interesse am religiösen Widerstand registrierte. ${ }^{297}$

Der Unterschätzung der potentiellen Bedeutung des Klerus ist es zuzuschreiben, dass die Ende 1971 beginnende Verschärfung der Repression gegen die religiöse Opposition weitgehend ignoriert wurde. Bereits im Jahr 1970 war ein junger Rechtsgelehrter und ehemaliger Schüler Khomeinis, Ājatollāh Mohammad Rezā Sa idi, vom SAWAK zu Tode gefoltert worden und in der Folgezeit war das Martyrium dieses Mannes zum Leitbild vieler der Nachfolgenden aufgestiegen. Sa idis Verbrechen war die öffentliche Kritik am Besuch einer amerikanischen Investorengruppe gewesen. ${ }^{298} \mathrm{Im}$ Jahr darauf erfolgte ein Angriff auf die Autonomie religiöser Institutionen mit dem Ziel, den bestehenden Widerstand zu zerstören und die finanzielle Handlungsfähigkeit der Kleriker zu beseitigen. Eine groß angelegte Kampagne richtete sich zudem nun direkt gegen Khomeini selbst im irakischen Exil, der als Landesverräter und Marionette Bagdads gebrandmarkte wurde. ${ }^{299}$ Vor einer Ermordung Khomeinis durch den SAWAK aber schreckte man offenbar zurück trotz vorhandener Kapazitäten - so war 1970 der ehemalige SAWAK-Chef Tajmur Baxtijār, nach Jahren des erzwungenen Exils im Anschluss an seine Absetzung 1961 inzwischen zur Exilopposition übergelaufen, durch iranische Agenten in Irak ermordet worden. ${ }^{300}$ Trotz der offenkundigen Aufmerksamkeit, die die iranischen Sicherheitsbehörden dem klerikalen Widerstand zuteil werden ließen, nahm man auf amerikanischer Seite das religiöse Phänomen nur am Rande war, wohl nicht zuletzt einem teleologischen Verständnis der zwangsläufigen Durchsetzung säkularer Vorstellungen im modernistischen Wandlungsprozess geschuldet. ${ }^{301}$

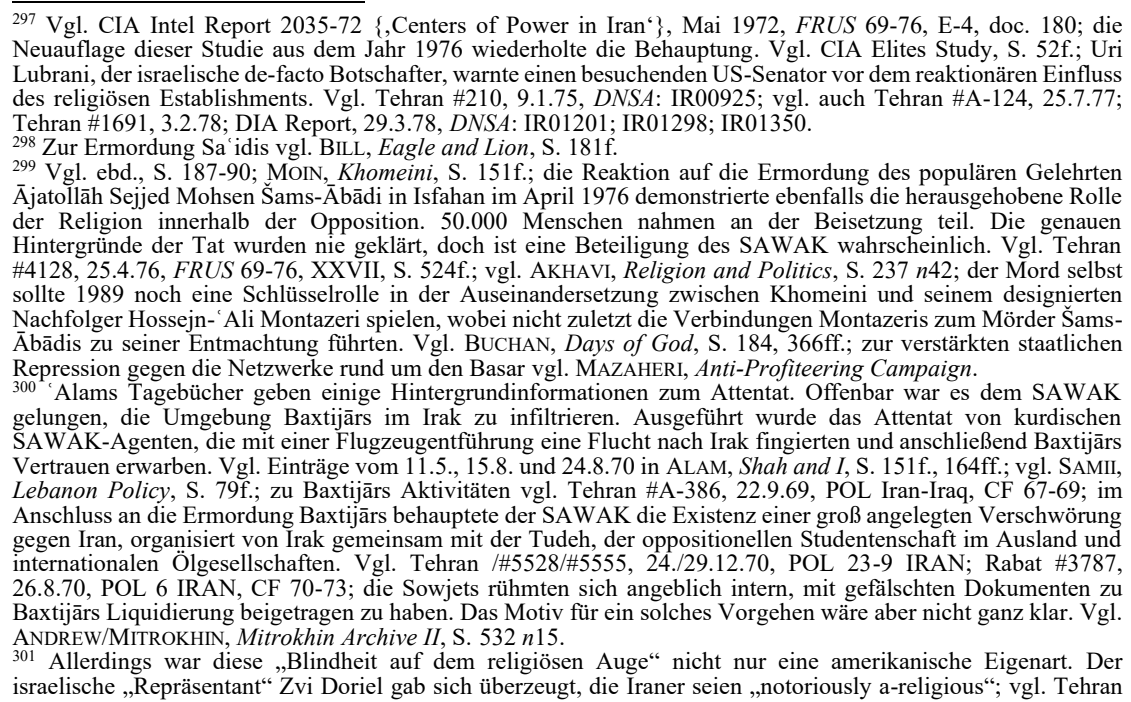


Die eigentliche Vorgeschichte der Iranischen Revolution selbst und die kumulierende Zuspitzung der innenpolitischen Krise im Anschluss an zögerliche Liberalisierungsversuche von oben nach 1976 befinden sich außerhalb des Rahmens dieser Studie. Einige abschließende Bemerkungen über die amerikanische Unfähigkeit, die ex post facto so offenkundig erscheinenden Vorzeichen der Revolution zu erkennen, sind jedoch angebracht. Alexis de Tocquevilles Diktum, gemünzt auf die Französische Revolution von 1789, wonach „,...] never was any such event, stemming from factors far back in the past, so inevitable yet so completely unforeseen“, ist durchaus auch adäquat für die Iranische Revolution. Charles Kurzman geht noch weiter und konstatiert in seiner Studie die (auch rückwirkende) Unmöglichkeit, die Revolution vorherzusehen. ${ }^{302}$ Angesichts der Schwierigkeit, die Vielzahl relevanter Faktoren für den letztendlichen Ausbruch der Revolution im Nachhinein zu gewichten, ist es zu einfach, die amerikanischen Planer in State Department, CIA und Pentagon bzw. die akademischen Experten für ihre Fehlannahmen über die Stabilität der politischen Ordnung zu verdammen. Bis heute gibt es unter Revolutions-Soziologen und Iranisten keine Einigkeit, welche Faktoren denn nun die wirkungsmächtigen für die Revolution waren. ${ }^{303}$ Wenn schon die retroaktive Vorhersage scheitert, ist ein pauschaler Vorwurf gegenüber den Zeitgenossen unangebracht. Ein späteres „Postmortem“ der CIA zum eigenen Versagen konstatierte lapidar, der iranische Fall, der Sturz eines Herrschers mit einem umfassenden und schlagkräftigen Sicherheitsapparat durch unbewaffnete Aufständische, sei letztlich präzedenzlos und damit unvorhersehbar gewesen. ${ }^{304}$

Die Unvorhersehbarkeit des späteren Zusammenbruchs in Rechnung stellend, so ist es dennoch berechtigt, die amerikanische Iranpolitik seit dem Zweiten Weltkrieg kritisch zu betrachten. Im Mittelpunkt früherer Forschungen steht dabei meist die herausgehobene amerikanische Rolle im Aufrüstungsprogramm des Schahs, das, so die häufig zu findende Lesart, letztlich hauptverantwortlich für die ökonomische wie soziale Schieflage Irans in den 1970er Jahren gewesen sei. Das mag in Ansätzen zutreffend sein, doch greift es sicherlich zu kurz als einziger oder auch nur entscheidender Explanans für die Revolution. ${ }^{305}$ Die in diesem und im

\footnotetext{
\#A-472, 6.3.68, POL IRAN ISR, CF 67-69; zur Unterschätzung des religiösen Widerstandes vgl. auch HOUGHTON, Three Images.

${ }^{302}$ Zit. aus Foran, Taking Power, S. 5; vgl. KuRZMAN, Unthinkable Revolution, S. 4-11.

303 Ähnlich die idiosynkratische Interpretation bei HALlidAY, Myth of Confrontation, S. 42-75; zuvor eher historisch-materialistisch in HALLIDAY, Genesis; aus der kaum noch zu überschauenden Literatur vgl. eine Zusammenfassung der verschiedenen Erklärungsansätze bei ARJOMAND, Turban for the Crown, S. 189-202; vgl. PARSA, Social Origins, S. 1-30; bedenkenswert die Thesen in KEDDIE, Can Revolutions Be Predicted; abzulehnen die vereinfachende Personalisierung bei SHAKIBI, Human Agency.

${ }^{304} \mathrm{Vgl}$. WOODWARD, Veil, S. 82.

${ }^{305}$ Einseitig die ökonomischen Ursachen der Revolution in den Vordergrund hebend LOONEY, Economic Origins; siehe auch ders., Military Expenditures, für das Argument, die Rüstungsausgaben seien hauptverantwortlich gewesen für den wirtschaftlichen Niedergang. Ähnlich HOVEYDA, Fall of the Shah, 77f.; Kissinger wehrte sich in der Retrospektive massiv gegen den Vorwurf, die exorbitanten Waffenkäufe nach 1972 hätten das wirtschaftliche Entwicklungsprogramm in Mitleidenschaft gezogen bzw. die politische Kohäsion des Landes beeinträchtigt. Vgl. KISSINGER, White House Years, S. 1260; vielmehr habe die Aufrüstung Irans externen Druck auf die innere Stabilität Irans abgewehrt und somit stabilisierend gewirkt. Bei aller berechtigten Kritik an den US-
} 
vorherigen Kapitel analysierten Entwicklungen lassen die Schlussfolgerung zu, dass auch ohne den kataklystischen Umbruch im Iran verstärkte Spannungen im bilateralen Verhältnis unausweichlich gewesen wären, zum Einen aufgrund der zunehmend konfligierenden Zielsetzungen beider Staaten in der Region, zum Anderen aufgrund des sich ausbreitenden Anti-Amerikanismus in breiten Schichten der Bevölkerung, dem auch eine in vielerlei Hinsicht vom Demos isolierten Herrschaftsklasse früher oder später hätte Rechnung tragen müssen. Das Scheitern der amerikanischen Iranpolitik - und nur wenige Autoren gestehen dieses Scheitern nicht ein $^{306}$ - ist eben nicht auf die Unfähigkeit zurückzuführen, sich einen grundsätzlichen Regimewechsel in Iran vorstellen zu können. Das eigentliche Scheitern liegt vielmehr in der Tatsache begründet, dass die US-Politik zu einer Verengung der eigenen Optionen führte und letzten Endes die Bindung an ein autoritäres und zunehmend unpopuläres Regime alternativlos und das Anwachsen anti-amerikanischer Überzeugungen unumkehrbar machte - die verzweifelten Versuche nach der Revolution, die eigene Rolle in den vorherigen fast vier Jahrzehnten $\mathrm{zu}$ negieren, um zumindest eine pragmatische Zusammenarbeit mit der Revolutionsregierung zu ermöglichen, illustrieren die vorherrschenden Verblendungen auf Seiten amerikanischer Entscheidungsträger in iranischen Belangen. ${ }^{307}$ Den fatalen langfristigen Folgen einer Identifikation der USA mit der Pahlawi-Herrschaft war man sich dabei, auch dies ein eindeutiges Ergebnis der vorliegenden Studie, durchaus bewusst. Letztlich aber überwog das Kalkül, die antiamerikanische Volksmeinung sei zu bändigen, solange die Monarchie das politische System Irans dominierte. Und zur Etablierung und zum Fortdauern dieser Dominanz hatten die permanenten Interventionen der USA seit dem Zweiten Weltkrieg entscheidend beigetragen. Es bedarf keiner zweifelhaften kontrafaktischen Spekulationen, um festzustellen, dass die Geschichte Irans in der zweiten Hälfte des 20. Jahrhunderts wohl um einiges anders verlaufen wäre, hätten sich die USA nicht als einflussreichste auswärtige Macht in Iran etabliert.

\footnotetext{
Waffenlieferungen ist Kissingers Verweis auf das Vorhandensein alternativer europäischer Waffenlieferanten in der Tat nicht von der Hand zu weisen.

306 In Ansätzen findet sich eine solche Argumentationsweise bei Kissinger, prägnant bei den vielen neokonservativen Interpretationen seit 9/11, wobei hier immer, wenn auch meist nicht offen ausformuliert, die Unterstützung des Schahs fürs Israel der eigentliche Antrieb für die Verteidigung der Pahlawi-Herrschaft ist. Exemplarisch eine frühe neo-konservative Interpretation LEDEEN/LEWIS, Debacle, S. 52; analog die Kritik an der Carter-Administration, da sie die notwendige gewaltsame Niederschlagung der Revolution verhindert habe. Vgl. ebd., S. 171-4, 236.

${ }^{307}$ Vgl. SICK, All Fall Down, S. 130-56; weniger deterministisch EMERY, US and Revolution; zur Politik der Carter-Administration in der Revolutionszeit vgl. auch GUERRERO, Carter and Pahlavi Dynasty.
} 